\title{
Probing the charge generation and recombination in thin-film, optoelectronic devices
}

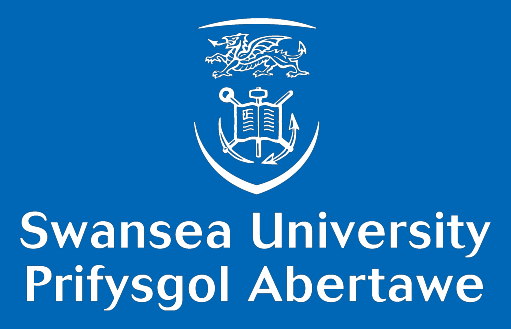

\author{
Stefan Zeiske
}

Department of Physics

Swansea University

Submitted to Swansea University in fulfilment of the requirements for the degree of

Doctor of Philosophy

2021 


\begin{abstract}
Sustainably and environment-friendly manufactured semiconductors are attractive candidates for next generation electronic and optoelectronic applications ranging from memory storage and computation, to power management and energy generation. In this regard, organic semiconductors, i.e., semiconductors based on conjugated carbon-based molecules and polymers derived from earth abundant elements, are the subject of intense basic research and technological development efforts. Understanding the fundamental processes governing these low-mobility and disordered semiconducting materials is therefore key to establish next generation applications based upon flexible and solution-processible organic semiconductors as global commercial technologies.

The work presented in this thesis focuses on the investigation of charge generation and recombination processes on thin film optoelectronic devices based upon organic semiconductors. A suite of experimental techniques, improved measurement setups, and expanded approaches are presented, and form the basis of comprehensive studies on state-of-the-art, high-efficiency organic photovoltaic systems. Specifically, an external quantum efficiency measurement technique with unprecedented dynamic range will be detailed. Using this enhanced apparatus, an approach allowing one to accurately determine charge generation quantum yields is introduced. After this, an extended technique to probe photogenerated charge carrier densities is outlined and applied to thin-film solar cells. Having emphasized the importance of studying charge generation, a combined theoretical and experimental exploration of the light intensity dependence of photocurrent and charge collection efficiency under the influence of various loss mechanisms is described. These insights provide the basis of a comprehensive study on organic solar cells, where recombination caused by localized trap states is found to be universally present under operational conditions limiting photocurrent and power-conversion efficiency. Overall, the work presented in this thesis expands on existing techniques and approaches, and yields important new understanding as to the device physics of thin-film, optoelectronic applications.
\end{abstract}




\section{Declaration}

This work has not previously been accepted in substance for any degree and is not being concurrently submitted for any degree.

Signed:

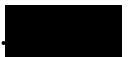
(candidate)

Date:

\section{August 2021}

This thesis is the result of my own investigations, except where otherwise stated. Where correction services have been used, the extent and nature of the correction is clearly marked in a footnote(s). Other sources are acknowledged by footnotes giving explicit references. A bibliography is appended.

Signed:

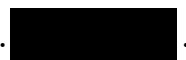
(candidate)

Date:

\section{August 2021}

I hereby give consent for my thesis, if accepted, to be available for photocopying and for inter-library loan, and for the title and summary to be made available to outside organisations.

Signed:

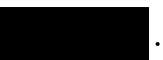
(candidate)

Date:

\section{August 2021}

The University's ethical procedures have been followed and, where appropriate, that ethical approval has been granted.

Signed:$$
\text { . }
$$
(candidate)

\section{August 2021}

Date: 


\section{Acknowledgements}

I would like to thank my supervisor, Prof. Paul Meredith, for the opportunity to undertake my research on thin-film, optoelectronic devices through the sustainable advanced materials research program at Swansea University. I am very thankful for all the support, guidance, and encouragement you have given me. Your invaluable advice, broad knowledge, years of experience, and unlimited assistance resulted in an excellent research environment. I also want to thank Dr. Ardalan Armin for his support, patience, and the inspiration he has given me. Without a doubt, you have expanded my horizons and taught me things that go well beyond science - I am humbled to have had learned from you as my mentor.

I would like to thank Dr. Oskar J. Sandberg for conducting drift-diffusion simulations and helping me understand the field of photovoltaics a bit better through equations; $D r$. Wei Li for fabricating optoelectronic devices; Dr. Nasim Zarrabi for assisting with measurements in the electro-optical laboratory; and Drew Riley for helping with experiments and proof-reading this thesis together with Dr. Gregory Burwell. I particularly want to thank Christina Kaiser - not only for helping with experiments and the many fruitful discussions we have had, but also for making my time at work and in Swansea more enjoyable.

A big thank you to Rhian Jones and Heather Evans for their endless help and support, organizing group events, and making university life a bit easier; Paul Hughes and Dr. Ryan Bigham for their assistance in the laboratory and clean room; and every member of the research program, particularly Dr. Farid Shahandeh and Dr. Sahar Basiri-Esfahani. 
I would like to thank all the people that I have met and had the opportunity to work with and learn from during my academic journey thus far, especially Prof. Dieter Neher, Prof. Safa Shoaee, Prof. Koen Vandewal, Dr. Jona Kurpiers, Lorena Perdigón-Toro, Dr. Mathias Nyman, Dr. Christian M. Wolff, Dr. Martin Stolterfoht, Dr. Elisa Collado-Fregoso, Dr. Malavika Arvind, Dr. Ulrich Hörmann, Dr. Fortunato Piersimoni, and Dr. Chung Man Fung.

I also want to thank my parents, Sonja and Jürgen, and my sister, Darja, who have given me unlimited support and love, and who are the anchors in my life - words are not enough to describe the deep gratitude I have knowing that I have you by my side. 


\section{Contents}

List of Figures xi

List of Tables xiii

List of Boxes

List of Symbols and Acronyms xvii

1 Theoretical background and fundamentals 1

1.1 Sustainable energy production, solar energy, and photovoltaics . . . . 2

1.2 Inorganic semiconductors . . . . . . . . . . . . . .

1.3 From inorganic to organic materials . . . . . . . . . . 9

1.4 Organic semiconductors . . . . . . . . . . . . . . 10

1.5 Working principle of an excitonic solar cell . . . . . . . . . . . 14

1.6 Basic device characterization . . . . . . . . . . . . . . . 19

1.7 Charge generation and geminate recombination . . . . . . . . . 24

1.7.1 From excitons to charge transfer states . . . . . . . . . . 26

1.7.2 From charge transfer states to free carriers . . . . . . . . 27

1.8 Charge collection and non-geminate recombination . . . . . . . . . 30

1.8.1 Bimolecular recombination and Langevin theory . . . . . . . . 32

1.8.2 Monomolecular recombination and trap states . . . . . . . . . 34

1.9 Motivation and scope . . . . . . . . . . . . . . . . 36

2 Sensitivity of sub-gap external quantum efficiency measurements 39

2.1 Sub-gap absorption in disordered semiconductors . . . . . . . . . . . 40

2.2 Basic considerations for sensitivity and dynamic range . . . . . . . . . 42

2.3 Home-built, ultra-sensitive EQE apparatus . . . . . . . . . . . . 44 
2.3.1 Dynamic range optimization . . . . . . . . . . . 47

2.3.2 The influence of optical bias on sensitivity . . . . . . . . . . 50

2.3.3 The impact of electrical bias on dynamic range . . . . . . . . 53

2.4 Ultra-sensitive EQE of photovoltaic devices . . . . . . . . . . 55

2.5 Conclusion . . . . . . . . . . . . . . . . . 57

3 Organic solar cells with near-unity charge generation yield 59

3.1 Charge generation in non-fullerene acceptor-based organic solar cells . . 60

3.2 Energetic offset and device performance . . . . . . . . . . . 63

3.3 Charge generation quantum yield . . . . . . . . . . . . 65

3.4 On the origin of strongly suppressed recombination . . . . . . . . . . 69

3.5 From thin- to thick-junction organic solar cells . . . . . . . . . . . 72

3.6 Organic solar cells with Shockley-type behaviour . . . . . . . . . . 76

3.7 Conclusion . . . . . . . . . . . . . . . . . 78

4 Low intensity integral time-of-flight technique 79

4.1 Debates on charge generation pathways . . . . . . . . . . . . 80

4.2 Experimental scheme of TDCF and LIITOF . . . . . . . . . . . . 82

4.3 Theoretical framework of LIITOF . . . . . . . . . . . . . . . 84

4.4 Limitations of LIITOF and how to address them . . . . . . . . . . . . 87

4.5 Field dependence of external generation efficiency . . . . . . . . . . . 90

4.6 Conclusion . . . . . . . . . . . . . . . . . . . . 94

5 Light intensity dependence of photocurrent 95

5.1 Photocurrent generation and collection efficiency . . . . . . . . . 96

5.1.1 Techniques to probe the photocurrent loss mechanisms . . . . . . 97

5.1.2 Basic considerations for charge collection efficiency . . . . . . . 99

5.2 Negligible recombination under ideal conditions . . . . . . . . . . . . 100

5.3 Bimolecular recombination and its impact on photocurrent . . . . . . 101

5.4 The effect of an external series resistance . . . . . . . . . . . . . . 103

5.5 The influence of space-charge effects . . . . . . . . . . . . 105

5.6 Recombination in the presence of trap states . . . . . . . . . 107

5.6.1 Trap depth estimation via point-of-transition approach . . . . . . 110

5.6.2 Trap depth estimation via quasi-Fermi level splitting approach . 111 
5.6.3 Trap density estimation via space-charge approach . . . . . . . 112

5.7 Simplified analysis for general recombination order . . . . . . . . . . 113

5.8 Sensitive IPC of thin-film, organic solar cells . . . . . . . . . . . . . 114

5.9 Conclusion . . . . . . . . . . . . . . . . . . . 118

6 Direct observation of trap-assisted recombination 121

6.1 Trap states in organic semiconductors . . . . . . . . . . . . . 122

6.2 Shockley-Read-Hall statistics: shallow and deep trap mode . . . . . . . 124

6.3 Universal presence of trap states . . . . . . . . . . . . . 126

6.4 Photoexcitation pathways of trap states . . . . . . . . . . . . 131

6.5 Trap states: electron acceptor- or donor-type? . . . . . . . . . . . . . 134

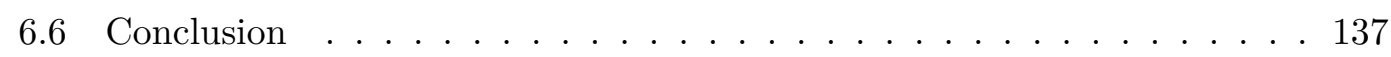

7 Summary and Outlook 139

A Device fabrication, statistics, and simulation model parameters 145

A.1 Chemical definitions . . . . . . . . . . . . . . . 146

A.2 Molecular structures . . . . . . . . . . . . . . . . 147

A.3 Device fabrication . . . . . . . . . . . . . . . . . . 148

A.3.1 Inverted organic solar cell architecture . . . . . . . . . . . 148

A.3.2 Conventional organic solar cell architecture . . . . . . . . . . 151

A.3.3 Perovskite solar cells . . . . . . . . . . . . . . . 153

A.3.4 Single-carrier organic devices . . . . . . . . . . . . 155

A.4 Device statistics and simulation model parameters . . . . . . . . 156

B Additional data and figures 161

Bibliography 
CONTENTS 


\section{List of Figures}

1.1 Origin of bandgaps in inorganic semiconductors . . . . . . . . . 5

1.2 Macroscopic and energetic structure of organic semiconductors . . . . 11

1.3 Working principle of an excitonic solar cell . . . . . . . . . . 15

1.4 Geminate and non-geminate recombination . . . . . . . . . . 16

$1.5 J-V$ characteristics, EQE spectrum, and Shockley-Queisser limit . . . . 22

1.6 Electronic states involved in charge generation processes . . . . . . . 24

1.7 Dissociation probability of $\mathrm{CT}$ states . . . . . . . . . . . 28

2.1 Home-built, ultra-sensitive EQE apparatus . . . . . . . . . . . 46

2.2 Optimization of EQE sensitivity . . . . . . . . . . . . . 48

2.3 Impact of photon noise on sensitivity . . . . . . . . . . . . . 51

2.4 Influence of light bias on dynamic range . . . . . . . . . . . . . 52

2.5 EQE under electrical bias . . . . . . . . . . . . . . . . . 53

2.6 Ultra-sensitive EQE of photovoltaic devices . . . . . . . . . . . 56

3.1 Energetics, $J$ - $V$ curves, and EQE spectra . . . . . . . . . . 63

3.2 Temperature dependent EQE spectra . . . . . . . . . . . . . 65

3.3 Energetics and kinetics of CT dissociation . . . . . . . . . . . . 67

3.4 Schematic of RPV setup and transients . . . . . . . . . . . . 69

3.5 RPV transients of PM6:Y6 and PM6:BTP-eC9 . . . . . . . . . . 70

3.6 Thickness dependent $J-V$ curves $\ldots \ldots \ldots \ldots$

3.7 Thickness dependent PV performance . . . . . . . . . . . . 73

3.8 Electro-optical simulations . . . . . . . . . . . . . . 74

3.9 Shockley-type and transport-limited solar cells . . . . . . . . . 76

4.1 LIITOF technique: Schematic and circuit diagram . . . . . . . . 82 


\section{LIST OF FIGURES}

4.2 TDCF technique: Schematic and circuit diagram . . . . . . . . . 83

4.3 Drift-diffusion simulations: $C(V)$ curves, intensity dependence, and LIITOF transients . . . . . . . . . . . . . . . . 88

$4.4 C(V)$ curves, voltage relations, and LIITOF transients . . . . . . . . 91

$4.5 \quad J-V$ curves and EGE results . . . . . . . . . . . . . . . . 92

$5.1 J_{\mathrm{sc}}$ and $\eta_{\mathrm{col}}$ under bimolecular recombination $\ldots \ldots \ldots \ldots$

$5.2 J_{\mathrm{sc}}$ and $\eta_{\mathrm{col}}$ under series resistance limitation $\ldots \ldots \ldots$

$5.3 J_{\mathrm{sc}}$ and $\eta_{\text {col }}$ under imbalanced mobilities . . . . . . . . . . . 106

$5.4 J_{\text {sc }}$ and $\eta_{\text {col }}$ under the presence of trap states $\ldots \ldots \ldots$

5.5 Point-of-transition (IPC) approach . . . . . . . . . . . . . . . . . 110

5.6 Quasi-Fermi level splitting approach . . . . . . . . . . . . . 111

5.7 Sensitive IPC of PM6:ITIC . . . . . . . . . . . . . . . . . . 114

5.8 Sensitive IPC of PBDB-T:EH-IDTBR . . . . . . . . . . . . 116

5.9 A simple guide for IPC analysis . . . . . . . . . . . . . . . . 119

6.1 Influence of trap states on IPC and $V_{\text {oc }} \ldots \ldots \ldots \ldots$

6.2 Experimental and simulated IPC and $V_{\mathrm{oc}} \ldots \ldots \ldots \ldots . \ldots . \ldots$

$6.3 \quad J-V$ curves and IPC characterizations . . . . . . . . . . . . . . 128

6.4 Statistics on trap energetics . . . . . . . . . . . . . . . . . 129

6.5 Probing trap states with IPC and ultra-sensitive EQE . . . . . . . . 131

6.6 Photoexcitation pathways of trap states and state energy diagram . . 132

6.7 SCLC results on single-carrier devices . . . . . . . . . . . . . . 134

6.8 Impact of solvent additive on trap energetics . . . . . . . . . . . 136

A.1 Molecular structures of organic semiconductors . . . . . . . . . . . 147

B.1 $J-V$ curve of PBDB-T:ITIC . . . . . . . . . . . . . . . . . . 162

B.2 Temperature dependent absorptance spectra . . . . . . . . . . . . 162

B.3 Refractive and extinction coefficients . . . . . . . . . . . 163

B.4 Thickness dependent EQE spectra . . . . . . . . . . . . . . . 163

B.5 RPV transient signals of organic solar cells . . . . . . . . . . . . . 164

B.6 IPC under exponential tail trap states . . . . . . . . . . . . . . 165

B.7 Simulated and experimentally obtained $J$ - $V$ curves $\ldots \ldots \ldots$

B.8 Trap depth determination: $\Delta_{\mathrm{t}, \mathrm{IPC}}$ versus $\Delta_{\mathrm{t}, \mathrm{QFLS}} \ldots \ldots \ldots \ldots$ 


\section{List of Tables}

1.1 Charge release times for different trap depths . . . . . . . . 35

5.1 IPC slope parameters for different photocurrent loss mechanisms . . . 119

A.1 Trap energetics of organic solar cells . . . . . . . . . . . . 156

A.2 PV parameters of organic solar cells . . . . . . . . . . . . . 156

A.3 Drift-diffusion model parameters . . . . . . . . . . . 157

A.4 Electro-optical device simulation parameters . . . . . . . . . . . 157

A.5 Thickness dependent PV parameters of PM6:Y6 . . . . . . . . . 158

A.6 Thickness dependent PV parameters of PM6:BTP-eC9 . . . . . . . . 158

A.7 Thickness dependent PV parameters of PM6:ITIC . . . . . . . . . . 158

A.8 Thickness dependent PV parameters of PBDB-T:EH-IDTBR . . . . . 159 


\section{List of Boxes}

\section{Additional information}

Box 0.1

Green boxes are used to provide additional and background information.

\section{Experimental details}

Box 0.2

Blue boxes are used to describe experimental methods and techniques.

\section{Conclusion}

\section{Box 0.3}

Red boxes are used to summarize important and general findings. 


\section{LIST OF BOXES}

2.1 Information to noise current . . . . . . . . . . . . . . . . 43

2.2 Details of lock-in method . . . . . . . . . . . . . . . . . 44

2.3 Improving the EQE dynamic range . . . . . . . . . . . . . . . . . 57

3.1 Current density versus voltage $(J-V)$ technique . . . . . . . . . . . 64

3.2 Temperature dependent absorptance technique. . . . . . . . . . . . . 66

3.3 Resistance dependent photovoltage (RPV) measurements . . . . . . . . . . 69

3.4 Steady-state double-injection (DoI) current experiments. . . . . . . . . . . 71

5.1 Steady-state intensity dependent photocurrent (IPC) technique . . . . . 115

5.2 Photocurrent loss mechanisms in IPC . . . . . . . . . . . . . . . . 119

6.1 Critical trap depth for photodetectors . . . . . . . . . . . . . . . 130

6.2 Sub-gap EQE fitting . . . . . . . . . . . . . . . . . . . . . 133

6.3 Space-charge limited current (SCLC) measurements . . . . . . . . . . 135

6.4 Trap energetics and morphology . . . . . . . . . . . . . . . . 136 


\section{List of Symbols and Acronyms}

$\begin{array}{ll}\alpha & \text { slope parameter, obtained from } J_{\mathrm{sc}} \text { versus } I_{\mathrm{L}} \\ \alpha_{\mathrm{FOM}} & \text { figure-of-merit } \\ \mathrm{A} & \text { acceptor } \\ \beta_{\mathrm{bulk}} & \text { bimolecular recombination coefficient } \\ \beta_{\mathrm{enc}} & \text { encounter rate coefficient } \\ \beta_{\mathrm{L}} & \text { Langevin recombination coefficient } \\ \beta_{\mathrm{SRH}} & \text { Shockley-Read-Hall (SRH) recombination coefficient } \\ C & \text { capacitance } \\ C_{\mathrm{geo}} & \text { geometrical capacitance } \\ C_{\mathrm{n}} & \text { electron capture coefficient } \\ C_{\mathrm{p}} & \text { hole capture coefficient } \\ \mathrm{CGY} & \text { charge generation quantum yield } \\ \mathrm{CT} & \text { charge transfer } \\ \mathrm{D} & \text { donor } \\ d & \text { layer thickness } \\ D_{\mathrm{e}}(E) & \text { electron density of states } \\ D_{\mathrm{h}}(E) & \text { hole density of states } \\ \mathrm{DOS} & \text { density of states } \\ \mathrm{DR} & \text { dynamic range } \\ \Delta E_{\mathrm{CT}} & \text { driving force for exciton dissociation } \\ \Delta f & \text { electrical bandwidth } \\ \Delta_{\mathrm{t}} & \text { trap depth } \\ E_{\mathrm{a}} & \text { activation energy } \\ E_{\mathrm{b}} & \text { binding energy } \\ E_{\mathrm{CT}} & \text { charge transfer state energy } \\ E_{\mathrm{H}, \mathrm{D}} & \text { donor HOMO energy } \\ E_{\mathrm{elec}} & \text { electric field } \\ E_{\mathrm{F}, \mathrm{n}} & \text { electron quasi-Fermi level } \\ E_{\mathrm{F}, \mathrm{h}} & \text { hole quasi-Fermi level } \\ E_{\mathrm{L}, \mathrm{A}} & \text { acceptor LUMO energy } \\ E_{\mathrm{S} 1} & \text { singlet excited state energy } \\ E_{\mathrm{t}} & \text { trap state energy } \\ & \end{array}$




$\begin{array}{ll}E_{\text {therm }} & \text { thermal energy } \\ E_{\mathrm{ph}} & \text { photon energy } \\ \text { EGE } & \text { external generation efficiency } \\ \text { EQE } & \text { external quantum efficiency } \\ \text { EQE }_{\text {exp }} & \text { experimentally obtained EQE } \\ \text { EQE }_{\text {min }} & \text { minimum detectable EQE } \\ \text { EQE }_{\text {max }} & \text { maximum detectable EQE } \\ \text { EQE }_{\mathrm{NE}} & \text { noise equivalent EQE } \\ e & \text { elementary charge } \\ \varepsilon & \text { dielectric constant } \\ \varepsilon_{0} & \text { vacuum permittivity } \\ \zeta & \text { bimolecular recombination reduction factor } \\ \eta_{\mathrm{abs}} & \text { absorption efficiency } \\ \eta_{\mathrm{CT}, \text { diss }} & \text { charge transfer state dissociation efficiency } \\ \eta_{\mathrm{E}, \mathrm{diff}} & \text { exciton diffusion efficiency } \\ \eta_{\mathrm{E}, \text { diss }} & \text { exciton dissociation efficiency } \\ \eta_{\mathrm{cc}} & \text { charge collection efficiency } \\ \eta_{\mathrm{overall}} & \text { overall efficiency } \\ \mathrm{FF} & \text { fill factor } \\ \gamma & \text { Langevin-reduction factor } \\ \gamma_{\mathrm{geo}} & \text { geometrical Langevin-reduction factor } \\ \gamma_{\mathrm{CT}} & \text { charge transfer state recombination probability } \\ G & \text { generation rate } \\ h & \text { Planck constant } \\ \hbar & \text { reduced Planck constant } \\ \phi_{\mathrm{A}} & \text { anode work-function } \\ \phi_{\mathrm{C}} & \text { cathode work-function } \\ \phi_{\mathrm{e}} & \text { electron flux } \\ \phi_{\mathrm{ph}} & \text { photon flux } \\ \mathrm{HOMO} & \text { highest occupied molecular orbital } \\ I_{\mathrm{L}} & \text { incident light intensity } \\ I_{\mathrm{NSD}} & \text { noise current spectral density } \\ I_{\text {noise }} & \text { noise current } \\ J_{\mathrm{G}} & \text { photogeneration current } \\ J_{\mathrm{G}, \mathrm{POT}} & \text { POT photogeneration current } \\ J_{\mathrm{MPP}} & \text { maximum power point current density } \\ J_{\mathrm{SC}} & \text { short-circuit current density } \\ J_{-V} & \text { current density versus applied bias voltage } \\ k_{\mathrm{B}} & \text { Boltzmann constant } \\ k_{\mathrm{d}} & \text { charge transfer dissociation rate } \\ k_{\mathrm{f}} & \text { charge transfer recombination rate } \\ k_{\mathrm{ij}} & \text { hopping rate } \\ k_{\mathrm{r}} & \text { bimolecular recombination rate } \\ & \end{array}$




$\begin{array}{ll}\lambda & \text { wavelength } \\ L_{\mathrm{D}} & \text { exciton diffusion length } \\ \text { LIITOF } & \text { low intensity integral time-of-flight } \\ \text { LUMO } & \text { lowest unoccupied molecular orbital } \\ \mu_{\mathrm{e}} & \text { electron mobility } \\ \mu_{\text {fast }} & \text { faster carrier mobility } \\ \mu_{\mathrm{h}} & \text { hole mobility } \\ \mu_{\text {slow }} & \text { slower carrier mobility } \\ n & \text { electron density } \\ n_{\text {id }} & \text { light ideality factor } \\ N_{\mathrm{C}} & \text { effective electron density of states } \\ N_{\mathrm{CT}} & \text { density of charge transfer states } \\ N_{\mathrm{L}, \mathrm{A}} & \text { density of transport states in acceptor LUMO } \\ N_{\mathrm{t}} & \text { trap density } \\ N_{\mathrm{V}} & \text { effective hole density of states } \\ p & \text { hole density } \\ P_{\text {in }} & \text { input power } \\ P_{\text {out }} & \text { output power } \\ \mathrm{PCE} & \text { power conversion efficiency } \\ \mathrm{POT} & \text { point-of-transition } \\ \Pi & \text { spatial disorder } \\ \sigma & \text { energetic disorder } \\ \mathrm{R} & \text { responsivity } \\ R & \text { recombination rate } \\ R_{\mathrm{S}} & \text { series resistance } \\ R_{\mathrm{S}, \text { eff }} & \text { effective series resistance } \\ R_{\mathrm{Shunt}} & \text { shunt resistance } \\ S & \text { slope parameter, obtained from EQE versus } I_{\mathrm{L}} \\ T & \text { temperature } \\ \mathrm{TDCF} & \text { time delayed collection field } \\ t_{\mathrm{delay}} & \text { delay time } \\ t_{\mathrm{RC}} & R C \text {-time } \\ t_{\mathrm{tr}} & \text { transit time } \\ \tau & \text { lifetime } \\ V_{\text {coll }} & \text { collection bias } \\ V_{\mathrm{dev}} & \text { device voltage } \\ V_{\mathrm{max}} & \text { maximal (photo-) induced change in voltage } \\ V_{\mathrm{MPP}} & \text { maximum power point voltage } \\ V_{\mathrm{OC}} & \text { open-circuit voltage } \\ V_{\mathrm{pre}} & \text { pre-bias } \\ & \end{array}$


List of Symbols and Acronyms 
Chapter 1

Theoretical background and fundamentals 


\section{THEORETICAL BACKGROUND AND FUNDAMENTALS}

\subsection{Sustainable energy production, solar energy, and pho- tovoltaics}

Initialized by the industrialization in the mid- $18^{\text {th }}$ century, the world has entered an ongoing period of extensive social, environmental, and economic change, which has not only led to revolutionary industrial advances and technological inventions, but also to an unprecedented growth in the world population [1, 2] and an associated, ever-higher rise in energy consumption. To date, fossil fuels are the predominant energy source covering most of the global energy demand [3, 4] - a situation that is currently questioned due to the effects of climate change. [5] 8] Many probabilistic projections of these imminent, anthropogenic threats forecast ecological long-term disasters - the extent of which can hardly be estimated - calling for rapid and target-oriented actions towards a more environmental-friendly and sustainable energy production. Renewable energy sources [also referred to as clean energies], that are, wind, solar and hydropower, are key engines in driving this dramatic change in the profile and means of energy production. By providing the energy to cover the global energy consumption a year within a fraction of a day, the sun is an almost endless source for solar energy - one of the most viable sources among the clean energies.

In general, solar energy can be converted to electrical power in three different ways, which are typically referred to as photovoltaic [i.e., converting solar energy directly into electrical power via an (internal) photoelectrical effect], photothermal [i.e., converting solar energy into heat that drives secondary steam generators, hence defining an indirect energy transformation], and photocatalytic [i.e., solar energy is used indirectly to produce electrical energy by promoting the catalysis of chemical reactions, which are, in turn, lead to reaction-product materials used to be burned and to drive steam generators] energy conversion. It is worth noting that only the photovoltaic energy conversion process can be considered as a direct one - devices, whose working principle is based upon them, are referred to as solar cells. While the photovoltaic effect [related to the photoelectrical effect, with the difference that photogenerated charges in the photoactive material are not ejected] was reported by Alexandre-Edmond Becquerel as early as 1839[9, 10], it took until 1954 before the first silicon solar cell was invented by Chapin et al.[11] at Bell Laboratories delivering power conversion efficiencies [a measure of how well a solar cell converts solar energy into electrical power] as 
high as $6 \%$. Since then, solar cells based upon silicon and other related, type III-V inorganic semiconducting materials were the subject of intense research, became the engine for various inventions and research fields, and currently dominate the industrialcommercial photovoltaic market with power conversion efficiencies breaking $25 \%$ in single-junction devices. 12, 13] However, given the large embodied manufacturing energy associated with processing crystalline semiconductors such as silicon accompanied with ever-growing global energy demands and protracted environmental pollution call for an environment-friendly alternative for future photovoltaic applications with lowembodied manufacturing energy, and a high degree of sustainability. In this regard, next-generation photovoltaics, that is, thin-film solar cells composed of organic and perovskite semiconducting materials, are an emerging class of solar cells showing great potential for industrial, large-area fabrication. While Pochettino discovered the electrical conductivity in the polymeric material Anthracene as far back as 1906[14] and the first fully-operational organic solar cell was reported as early as 1975 15] [with a power conversion efficiency as small as $0.001 \%]$, tremendous progress, and remarkable highefficiency, and inorganic photovoltaic-competitive power conversion efficiencies of close to $20 \%$ have been achieved only recently. It is also true that the disordered nature of organic semiconducting materials, high permittivity-related incomplete free charge generation at room temperature, and low carrier mobilities in organic, conjugated systems are not only properties differentiating organic from inorganic semiconductors, but also complicating and limiting the adaption of measurement techniques, previously developed to investigate solar cells based upon crystalline, high-mobility inorganic materials, to organic optoelectronics.

In this chapter, the general status of the current understanding of basic, solid-state physics of crystalline, inorganic semiconductors is reviewed at first, before the class of organic semiconducting materials is introduced and discussed. Equipped with fundamentals in the working principle of excitonic solar cells, and basic photovoltaic device characterization, the subtle links between device efficiency and electro-optical phenomena are highlighted. Based upon those essential relations, generation and recombination processes of charge carriers in organic solar cells are elucidated. 


\section{THEORETICAL BACKGROUND AND FUNDAMENTALS}

\subsection{Inorganic semiconductors}

Inorganic semiconductors, such as germanium [Ge] and silicon $[\mathrm{Si}]$, consist of a periodic, ordered crystal structure, often referred to as lattice, with atoms covalently bonded to one another. As the atomic mass of the core is much larger than that of electrons, the dynamics and bonding of these systems are dominated by the outermost electrons, known as valence electrons [Born-Oppenheimer approximation 16]]. Here, the distance between the nearest-neighbour atoms [referred to as lattice constant, a] is small enough for the wave functions of the involved electrons to overlap, and interact with one another. Electrons are fermions [i.e., particles with a half-integer spin], hence the occupation of energy states follows the Fermi-Dirac statistics based on the spin-statistical Pauli exclusion principle [17] [i.e., half-integer particles cannot occupy the same energetic state characterized by a certain orbital $(n)$, angular momentum $(l)$, magnetic $(m)$, and spin $(s)$ quantum number]. To evaluate the energetic landscape of a lattice-structured semiconductor it is conventional to contemplate the available momentum spectra of valence electrons. Considering an infinite one-dimensional lattice of equally spaced and fixed atomic cores in $k$-space [also referred to as reciprocal space, or momentum space, which can be understood as a Fourier-transform of a Bravais lattice typically used in solid state physics to define crystalline arrangements [18] and approximating the valence electron energy as isoenergetic parabola leads to overlaps in the allowed valence-states between neighbouring atoms as seen in Fig. 1.1 a.

Due to Pauli's exclusion principle, emerging intersections of isoenergetic states are forbidden - hence, those states are separated [i.e., energetically lifted and lowered], and a forbidden energy band is formed [see Fig. 1.1 b]. Applying the Drude-Sommerfeld model to semiconducting materials, the energy of states allowed to be occupied by electrons, can be estimated via the particle-in-a-box model, in which electrons are treated as an ideal Fermi gas [defined as an ensemble of delocalized and non-interacting fermions] in a perfectly ordered lattice with infinite-periodic potential $[V(r) \propto V(k)=V(k+G)$, where $G$ denotes the reciprocal period lattice constant]. Here, motivated by the delocalization character, electrons are described mathematically as wavefunctions $\Psi_{\mathrm{k}}(r)$ with the corresponding Hamiltonian $\hat{H}=-\frac{\hbar^{2}}{2 m_{\mathrm{e}}} \nabla^{2}+V(r)\left[m_{\mathrm{e}} \approx 9.109 \ldots \times 10^{-31} \mathrm{~kg}\right.$ is the electron mass, and $\hbar \approx 6.582 \ldots \times 10^{-16} \mathrm{eVs}$ denotes the reduced Planck constant]. The 
Hamiltonian $\hat{H}$ is composed of an infinite-periodic potential [most prominently simplified as infinite-periodic, rectangular potential barriers in the Kronig-Penny model], and a kinetic term [containing the electron momentum, $p=\hbar k$ ]. The corresponding time-independent Schrödinger equation is then given by 18

$$
\left[-\frac{\hbar^{2}}{2 m_{\mathrm{e}}} \nabla^{2}+V(r)\right] \Psi_{\mathrm{k}}(r)=E_{\mathrm{k}} \Psi_{\mathrm{k}}(r)
$$

where $E_{\mathrm{k}}$ denotes the allowed electronic states, known as eigenstates. In accordance with the Bloch theorem, the solution of this Schrödinger equation is given by so-called Bloch waves $\left[\Psi_{\mathrm{k}}(r)=\exp (i k r) \times u(r)\right.$ with $u(r)=u(r+R)$, where $R$ denotes a translation vector of the corresponding Bravais lattice] composed of a kinetic $[\exp (i k r)]$ and infinite-periodic $[u(r)]$ term.
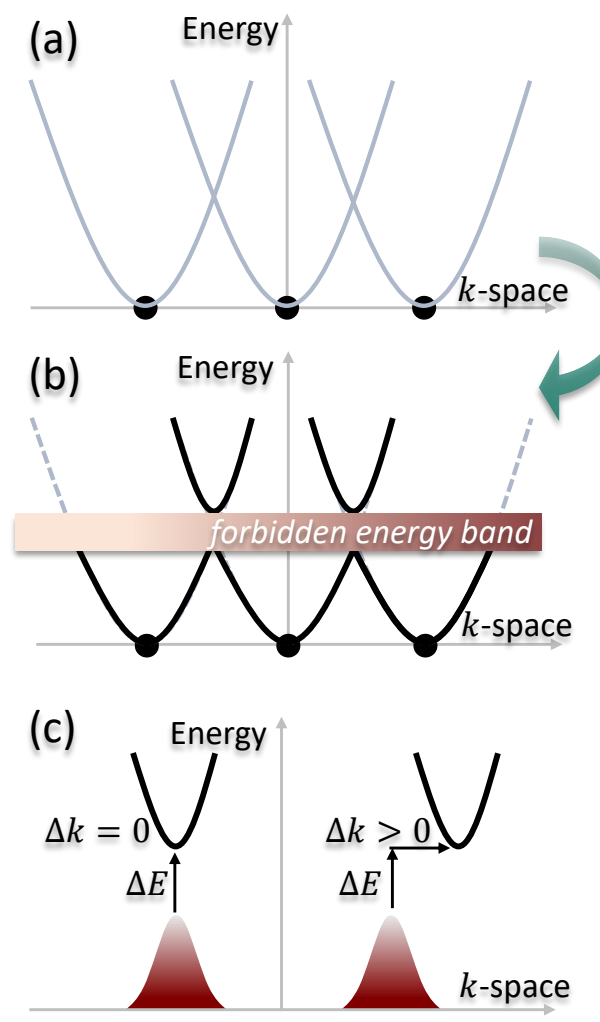

Figure 1.1: (a) Schematic of a one-dimensional lattice of equally spaced atom cores with electron isoenergetic parabolic lines in the energy $-k$ space. (b) Electron-electron coupling under consideration of Pauli's exclusion principle leads to the formation of forbidden energy bands. (c) Schematic of a direct [left] and indirect [right] bandgap semiconductor in the energy - $k$ space. 


\section{THEORETICAL BACKGROUND AND FUNDAMENTALS}

Based upon Pauli's principle, the energetic solutions to Eq. 1.1, that are, $E_{\mathrm{k}}=$ $[\hbar k]^{2}\left[2 m_{\mathrm{e}}\right]^{-1}$, are filled from low to high energy with the ensemble of all unoccupied [occupied] energy states defined as the conduction [valence] band. The energy difference between valence band maximum [of energy $E_{\mathrm{V}}$ ] and conduction band minimum [of energy $\left.E_{\mathrm{C}}\right]$ is referred to as bandgap, and has an energy $E_{\mathrm{g}}=E_{\mathrm{C}}-E_{\mathrm{V}}$. Based upon the minimum energy $\Delta E$ [also referred to as fundamental energy] and additional momentum $\Delta k$ [typically provided via interaction with the lattice vibrations, so-called phonons] required for a transition from valence band maximum to conduction band minimum, direct $[\Delta k=0$; e.g., gallium arsenide (GaAs), or indium arsenide (InAs)] and indirect bandgaps $[\Delta k>0$; e.g., Si, or Ge] are distinguished [see Fig. 1.1c] . At $T=0 \mathrm{~K}[$ where enthalpy and entropy of a system are minimum], all energy states $E_{\mathrm{k}}$ with $k<k_{\mathrm{F}}$ $\left[k>k_{\mathrm{F}}\right.$ ] are occupied [unoccupied]. $E_{k_{\mathrm{F}}}$ [or simplified $E_{\mathrm{F}}$ ] denotes the Fermi energy of the lattice and is defined as the energy at which the probability of electronic state occupation $[f(E)]$ is $1 / 2$ [for any $T>0 \mathrm{~K}$ also referred to as Fermi level]. In general, this electron occupation probability $f(E)$ of a state of energy $E$ at a given temperature $T$, follows the Fermi-Dirac statistics, which can be expressed as: 19

$$
f(E)=\left\{\exp \left[\frac{E-E_{\mathrm{F}}}{k_{\mathrm{B}} T}\right]+1\right\}^{-1}
$$

where $k_{\mathrm{B}} \approx 8.617 \ldots \times 10^{-5} \mathrm{eV} \mathrm{K}{ }^{-1}$ denotes the Boltzmann constant. It is worth noting that the energetic [and spatial] form and magnitude of allowed energy states [referred to as density of states, or DOS] fundamentally depend on the lattice structure and atomic arrangements. Further, the DOS can be distorted by structural defect states originating from randomly distributed impurities, which cause both lattice symmetry and electron delocalization to break. [20] The electron [hole] DOS, $D_{\mathrm{e}}(E)\left[D_{\mathrm{h}}(E)\right]$, defines the number of states at energy $E$ per unit energy and unit volume, and can be expressed as:

$$
D_{\mathrm{e}}(E)=4 \pi\left[\frac{2 m_{\mathrm{e}}^{*}}{h^{2}}\right]^{3 / 2} \sqrt{E-E_{\mathrm{C}}}
$$

and

$$
D_{\mathrm{h}}(E)=4 \pi\left[\frac{2 m_{\mathrm{h}}^{*}}{h^{2}}\right]^{3 / 2} \sqrt{E_{\mathrm{V}}-E} .
$$

Note that the DOS-to-energy relation as represented in Eq. 1.3 and 1.4, reflects a 
three-dimensional consideration only, and is expected to change to $D(E)=$ const. for two-, and $D(E) \propto[\sqrt{E}]^{-1}$ for one-dimensional considerations. Under the assumption of $E-E_{\mathrm{F}}>k_{\mathrm{B}} T$ [i.e., when the (quasi-) Fermi level is well distanced from the transport band edge corresponding to the case of moderate temperatures and/or low doping concentration; often referred to as non-degenerate semiconductors], the Fermi-Dirac statistics can be approximated by the Boltzmann statistics $f_{\text {Boltzmann }}=\exp \left\{\left(E_{\mathrm{F}}-\right.\right.$ $\left.E)\left[k_{\mathrm{B}} T\right]^{-1}\right\}$. The free electron [hole] density $n[p]$ in a conduction [valence] band noting that the $n[p]$ is much less than the total number of available conduction [valence] band states - can then be expressed as:

$$
n=\int_{E_{\mathrm{C}}}^{\infty} D_{\mathrm{e}}(E) f(E) d E=N_{\mathrm{C}} \exp \left[\frac{E_{\mathrm{F}, \mathrm{e}}-E_{\mathrm{C}}}{k_{\mathrm{B}} T}\right]
$$

and

$$
p=\int_{-\infty}^{E_{\mathrm{V}}} D_{\mathrm{h}}(E)[1-f(E)] d E=N_{\mathrm{V}} \exp \left[\frac{E_{\mathrm{V}}-E_{\mathrm{F}, \mathrm{h}}}{k_{\mathrm{B}} T}\right],
$$

where $N_{\mathrm{C}}$ and $N_{\mathrm{V}}$ denote the electron and hole effective density of states [typically referred to as the DOS at the corresponding transport band edges], and are given by:

$$
N_{\mathrm{C}}=2\left[\frac{2 \pi m_{\mathrm{e}}^{*} k_{\mathrm{B}} T}{h^{2}}\right]^{3 / 2}
$$

and

$$
N_{\mathrm{V}}=2\left[\frac{2 \pi m_{\mathrm{h}}^{*} k_{\mathrm{B}} T}{h^{2}}\right]^{3 / 2}
$$

Here, $m_{\mathrm{e}}^{*}$ and $m_{\mathrm{h}}^{*}$ denote the effective mass of electrons and holes, which are generally related to the form of the isoenergetic parabolas in $k$-space [see Fig. 1.1 a, b] via $1 / m^{*}=\hbar^{-2}\left[\partial^{2} E / \partial k^{2}\right]$. The law of mass action relates the electron and hole concentrations to one another and can be stated as:

$$
n p=n_{\mathrm{i}}^{2} \exp \left[\frac{E_{\mathrm{F}, \mathrm{e}}-E_{\mathrm{F}, \mathrm{p}}}{k_{\mathrm{B}} T}\right]=N_{\mathrm{C}} N_{\mathrm{V}} \exp \left[-\frac{E_{\mathrm{g}}}{k_{\mathrm{B}} T}\right] \exp \left[\frac{E_{\mathrm{F}, \mathrm{e}}-E_{\mathrm{F}, \mathrm{p}}}{k_{\mathrm{B}} T}\right],
$$

where $n_{\mathrm{i}}$ denotes the intrinsic charge carrier density. The combination of low bandgaps [e.g., $0.35 \mathrm{eV}$ (InAs), $0.67 \mathrm{eV}(\mathrm{Ge}), 1.1 \mathrm{eV}(\mathrm{Si})$, or $1.4 \mathrm{eV}(\mathrm{GaAs})][18,21]$ and large dielectric constants [e.g., $\varepsilon \approx 15(\mathrm{InAs}), \varepsilon \approx 16(\mathrm{Ge}), \varepsilon \approx 12(\mathrm{Si})$, or $\varepsilon \approx 13(\mathrm{GaAs})][18]$ in 


\section{THEORETICAL BACKGROUND AND FUNDAMENTALS}

inorganic, crystalline semiconductors ultimately leads to a room temperature-activated dissociation of bound electron-hole pairs after excitation via external stimulus. As a direct consequence, the intrinsic conductivity $\sigma=e n \mu\left[e \approx 1.602 \ldots \times 10^{-19} \mathrm{C}\right.$ is the elementary charge and $\mu$ denotes the free carrier mobility typically in the unit $\mathrm{cm}^{2} \mathrm{~V}^{-1} \mathrm{~s}^{-1}$ ] of inorganic semiconductors lies between $10^{-8}$ and $10^{-2} \Omega^{-1} \mathrm{~cm}^{-1}$. Conductors [insulators], in turn, have bandgap energies of $E_{\mathrm{g}} \approx 0 \mathrm{eV}\left[E_{\mathrm{g}}>3 \mathrm{eV}\right]$, with $E_{\mathrm{F}}$ at $T=0$ $\mathrm{K}$ situated within the conduction band [right in the middle of the bandgap] exhibiting conductivities above [below] $10^{-2}\left[10^{-8}\right] \Omega^{-1} \mathrm{~cm}^{-1}$. The high conductivities in inorganic semiconductors result, inter alia, from high electron $\left[\mu_{\mathrm{e}}\right]$ and hole $\left[\mu_{\mathrm{h}}\right]$ mobilities, e.g., $\mu_{\mathrm{e}} \approx 1400 \mathrm{~cm}^{2} \mathrm{~V}^{-1} \mathrm{~s}^{-1}$ and $\mu_{\mathrm{h}} \approx 500 \mathrm{~cm}^{2} \mathrm{~V}^{-1} \mathrm{~s}^{-1}[\mathrm{Si}], \mu_{\mathrm{e}} \approx 3900 \mathrm{~cm}^{2} \mathrm{~V}^{-1} \mathrm{~s}^{-1}$ and $\mu_{\mathrm{h}} \approx 1900 \mathrm{~cm}^{2} \mathrm{~V}^{-1} \mathrm{~s}^{-1}[\mathrm{Ge}]$, or $\mu_{\mathrm{e}} \approx 40000 \mathrm{~cm}^{2} \mathrm{~V}^{-1} \mathrm{~s}^{-1}$ and $\mu_{\mathrm{h}} \approx 500 \mathrm{~cm}^{2} \mathrm{~V}^{-1} \mathrm{~s}^{-1}$ $[\operatorname{InAs}] \cdot[18]$ 


\subsection{From inorganic to organic materials}

Silicon is one of the most-studied semiconducting material systems in the world and, when used as $p$ - and $n$-doped compounds [doping refers to the controlled introduction of impurities into an intrinsic (i.e., undoped) semiconductor with the aim to modify electrical properties, such as conductivity] in a p-n junction, a prominent representative of solar cells - devices that convert solar energy into electrical power. As such, single-junction crystalline [amorphous] Si-based solar cells currently reach efficiencies as high as $26.7 \%[12$ [ [ $>10 \%[13]$ ]; single-junction GaAs-based solar modules recently broke the $28 \%[13]$ limit. However, the manufacturing processes of inorganic solar cells are expensive and highly energy- and time-demanding due to special purification procedures, high processing temperatures, and complex crystal growing techniques. Hence, a new generation of photovoltaic systems, that is, solar cells based upon organic semiconductors attracted attention in the photovoltaic community. Organic materials are easy to synthesize, low weighted, often solution-processed, offer mechanical flexibility, and are much cheaper to fabricate. Thus, organic photovoltaic applications offer an attractive and economy-friendly alternative to their inorganic-based counterparts. In particular, the high absorption coefficients of organic semiconductors enable the fabrication of thin, yet absorption-efficient optoelectronic devices. While advances in organic chemistry and polymer engineering allow for almost endless variability in organic semiconductor properties, the mechanical flexibility and low-cost fabrication, in turn, open completely new markets for organic photovoltaic applications, e.g., the integration of organic solar cells, light emitting diodes, and photodetectors into buildings, vehicles, and clothing. 


\section{THEORETICAL BACKGROUND AND FUNDAMENTALS}

\subsection{Organic semiconductors}

In contrast to their inorganic crystalline counterparts, organic semiconductors are hydrocarbon compounds that feature a $\pi$-electron system, and are categorized as the group of polymers and materials of low-molecular weight, often referred to as small molecules, or molecular semiconductors. The $\pi$-electron system results from interacting $p_{\mathrm{z}}$-orbitals of nearest neighbouring carbon atoms via alternating single and multiple [most commonly, double] bonds [see Fig. 1.2a]. The molecular backbone, however, is based on $\sigma$-bonds, which result from the $\left[s p, s p^{2}\right.$ and $\left.s p^{3}\right]$ hybridization of carbon atoms [i.e., the $2 s$ and $2 p$ carbon atom orbitals form, according to Hund's rules, up to four energetically equal hybrid orbitals], and are much stronger than the $\pi$ counterpart bonds due to their lower coupling strength. To determine the allowed energy states in an organic semiconductor, the $\pi$-electron systems can be approximated via the particle-in-a-box approach, where the length of the box $L=a N$ is determined by the [average] carbon bond length $a$ between $N$ carbon atoms. Solving the corresponding time-independent Schrödinger equation results in eigenstates with energy $E_{\mathrm{n}}=h^{2} n^{2}\left[8 m_{\mathrm{e}} L\right]^{-1}\left[h=\hbar 2 \pi \approx 6.626 \ldots \times 10^{-34} \mathrm{~m}^{2} \mathrm{~kg} \mathrm{~s}^{-1}\right.$ denotes the Planck constant $]$. The interaction of the involved carbon valence electron wavefunctions, which can be understood as constructive and deconstructive interference, is associated with the formation of energetically lower lying $\pi$ and $\sigma$ orbitals [referred to as bonding orbitals], and energetically higher lying, hence unfavoured, orbitals [referred to as anti-bonding $\pi^{*}$ and $\sigma^{*}$ orbitals]. While the lowest $N / 2$ states $[\pi$ and $\sigma$ orbitals] are occupied by electrons [where the highest occupied orbital is referred to as the highest occupied molecular orbital, or HOMO], the energetically higher lying $N / 2$ states $\left[\pi^{*}\right.$ and $\sigma^{*}$ orbitals] remain unoccupied [referred to as the lowest unoccupied molecular orbital, or LUMO]. Analogous to inorganic semiconductors, the energy gap [or, bandgap] in organic semiconductors is defined as the difference between the HOMO and LUMO levels $\left[E_{\mathrm{g}}=h^{2}\left[8 m_{\mathrm{e}} a^{2} N\right]^{-1} \sim[L]^{1}\right]$ [see Fig. 1.2 $\left.\mathbf{b}\right]$. It is worth noting that the HOMO and LUMO in molecular solids are not 'real' bands [and so is the bandgap], but rather localized eigenstates of the corresponding Schrödinger equation - for convenience in this thesis, however, the phrase 'bandgap' will be adopted as the energy difference between HOMO and LUMO energies. The energetic position of the HOMO and LUMO level 
[and subsequently, the bandgap $E_{\mathrm{g}}$ ] depends on influential, functional end- and sidegroups of the polymer and, in particular, on the length $L$ of the conjugated system. It is, however, worth noting that this one-dimensional quantum well model is a simplified approximation only, and while it can explain the decrease in the bandgap energy of disordered organic semiconductors for an increasing number of involved carbon atoms $N$ [respectively $\pi$-bonds] [e.g., Ethylene $\left(N=2, E_{\text {g,optical }} \approx 6.7 \mathrm{eV}\right) \rightarrow 1.3$ Butadiene $\left(N=4, E_{\mathrm{g}, \text { optical }} \approx 5.8 \mathrm{eV}\right) \rightarrow 1.3 .5$ Hexatriene $\left.\left(N=6, E_{\mathrm{g}, \text { optical }} \approx 4.8 \mathrm{eV}\right)\right]$, it fails for large $N$ [e.g., Poly(acetylene)]. Here, at large enough $N$ the one-dimensional model predicts $E_{\mathrm{g}} \rightarrow 0$ contrary to experimental observations. This discrepancy can be explained by Peierls instability theorem, which can be understood as a distortion of a [molecular] lattice by weak molecule-vibrations leading to a lattice constant transition from $a$ to $2 a$, and hence a formation of new transport bands [energies].

(a) Single conjugated segment
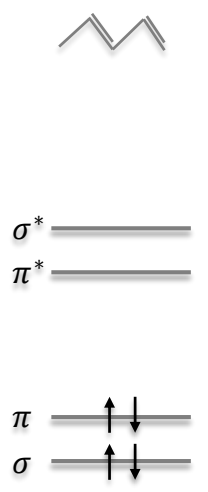

(b) Conjugated segment chain
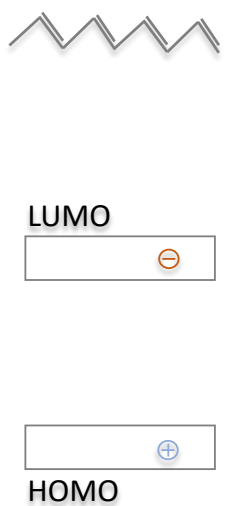

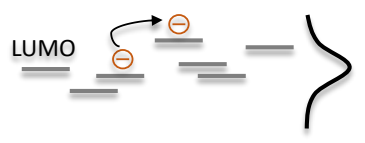

(c)

Disordered semiconductor
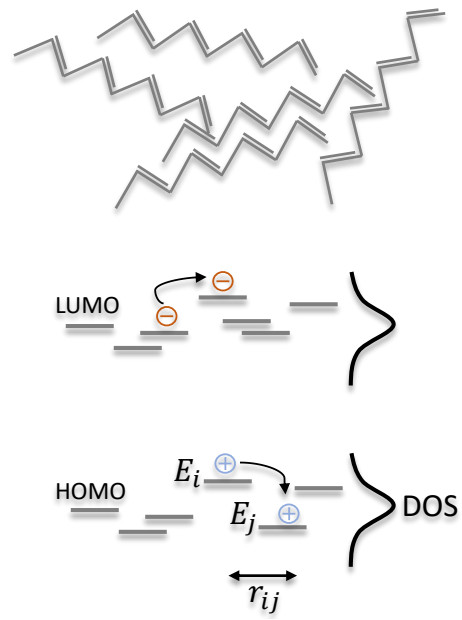

Figure 1.2: (a) Simplified representation of macroscopic and energetic structure of a single conjugated segment with single and double bonds, as well as bonding and antibonding $\sigma$ - and $\pi$-, respectively $\sigma^{*}$ - and $\pi^{*}$-, bonds. (b) A chain of multiple conjugated segments is displayed, where the intra-chain transport of electrons and holes takes place via band-like transport in HOMO and LUMO energy bands. (c) Schematic picture of an organic, disordered semiconductor composed of several weakly Van der Waals bonded, conjugated segment chains. The inter-chain transport of charge carriers in-between the single segment chains takes place via hopping. The energetic distribution of LUMO and HOMO levels is summarized by a Gaussian density of states [DOS]. 


\section{THEORETICAL BACKGROUND AND FUNDAMENTALS}

A more reliable and accurate method to determine the energy of molecular orbitals is the so-called LCAO-MO approach - linear combination of atom orbitals to molecular orbitals. 18, 21 Here, a molecular orbital, $\Psi_{\mathrm{k}}=\sum_{\mathrm{i}} c_{\mathrm{i}} \phi_{\mathrm{i}}$, is expanded as a superposition of involved atomic orbitals, $\phi_{\mathrm{i}}$, which are differently weighted through a pre-factor $c_{\mathrm{i}}$. Solving the corresponding 'Schrödinger-like' equation [specifically, defined as KohnSham equation] and occupying the molecular orbitals with electrons in accordance with Pauli's and Hund's principle ultimately allows the construction of molecular orbitals, and the estimation of the HOMO and LUMO energy values accordingly.

Similar to an inorganic semiconductor, where the bandgap is a property of the crystal, the HOMO-LUMO bandgap in an organic semiconductor is an intrinsic property of the corresponding conjugated system. The energetic disorder inducing irregular structure of thin, organic semiconducting materials [typically amorphous, or polycrystalline] accompanied by relatively weak van der Waals forces, however, does not allow for the formation of well-defined, broad energy bands as it is the case in inorganic semiconductors. On the contrary, charges in organic semiconducting materials are localized to single molecules. With [optical] bandgaps of 1.5 to $3 \mathrm{eV}$ and dielectric constants as low as $\varepsilon \approx 3$, the energy required to generate free charges in organic semiconductors [i.e., to overcome the Coulomb attraction between the LUMO electron and HOMO hole] is much larger compared to their inorganic semiconductor counterparts. Assuming a dielectric constant of $\varepsilon=3$ and a diameter of $r=1 \mathrm{~nm}$, the electron-hole binding energy, $E_{\mathrm{b}}=e^{2}\left[4 \pi \varepsilon \varepsilon_{0} r\right]^{-1}$, is approximately $500 \mathrm{meV}$, and thus much larger than thermal energy at room temperature $[\sim 25 \mathrm{meV}]$. Therefore, upon excitation charges form bound electron-hole pairs known as excitons. Hence, an additional stimulus [typically in form of an electric field, or thermal excess energy] is required to overcome the Coulomb attraction. Given the nature of organic semiconductors [typically treated as a collective of single molecular and polymer units] with charge carriers localized to single units only, the DOSs of single conjugated systems within the ensemble can be mathematically described via multiple, single delta functions [corresponding to a zero-dimensional treatment in $k$-space]. The ensemble of all [energetically distributed] zero-dimensional DOSs is typically assumed to be either Gaussian or exponential, and can be expressed accordingly as: 


$$
D_{\text {gauss }}(E)=\frac{N}{\sigma_{\text {gauss }} \sqrt{2 \pi}} \exp \left[-\frac{E^{2}}{2 \sigma_{\text {gauss }}^{2}}\right]
$$

and

$$
D_{\exp }(E)=\frac{N}{\sigma_{\exp }} \exp \left[-\frac{E}{\sigma_{\exp }}\right]
$$

where $N$ refers the effective density of states [typically on the order of $10^{20} \mathrm{~cm}^{-3}$ ], while $\sigma_{\text {gauss }}$ and $\sigma_{\exp }$ each specify the width of the Gaussian and exponential distribution [often referred to as (energetic) disorder] [see Fig. 1.2. c].

The molecular structure of organic semiconductors, in particular the side- and endchains [typically combinations of heteroatoms such as nitrogen, sulphur, and oxygen] as well as core units, strongly affect the electronic properties, such as $\pi-\pi$ stacking enhancing [electron] mobility, or high electron affinity conferring moieties [e.g., chlorine, or fluorine], or HOMO and LUMO energy levels. Depending on the structural, energetic, optical, and electronic properties organic semiconductors are classified into two categories; electron-donating units [typically referred to as donor] and electron-accepting units [typically referred to as acceptor]. In this regard, it is possible to design molecules tailored to specific needs though modifications of the chemical structure. While in the early stage of organic photovoltaic applications, most acceptor-like semiconductors were based on the $C_{60}$-fullerene and derivatives [with energy orbitals and optical properties difficult to tailor due to their stiff 'ball-like' molecular base structure], more complex, three-dimensional, and non-fullerene based semiconducting molecules are dominating the organic acceptor landscape today. $[22$

In the following section, the basic working principle of an organic solar cell, that is, a light-harvesting devices composed of a blend of different organic donor and acceptor semiconductors, will be reviewed. 


\section{THEORETICAL BACKGROUND AND FUNDAMENTALS}

\subsection{Working principle of an excitonic solar cell}

The basic working principle of any solar cell is to transform a flux of photons of energy $E_{\mathrm{ph}}$ into a flux of charge carriers, which can be extracted at the electrodes. Hence, the overall efficiency [referred to as power conversion efficiency] of any [organic] solar cell is ultimately determined by the balance between photon-absorption and carrier extraction. The initial photon absorption and subsequent charge-related processes, such as generation and transport, take place in a photoactive layer [commonly containing multiple organic semiconducting materials] sandwiched between two external circuit forming terminals [i.e., charge-selective electrodes]. To allow incoming light to enter the photoactive layer, one of the electrodes [ordinarily the anode, as it is the case in solar cells with conventional device architecture] needs to be a semi-transparent, conductive material, e.g., indium tin oxide [ITO]. As mentioned above, the exciton binding energy in organic semiconductors usually cannot be overcome thermally [at room temperature], which makes so-called homojunction solar cells [where the photoactive layer consists of a single organic semiconducting material only] very inefficient. In 1986, Tang and coworkers introduced the concept of so-called heterojunction solar cells [23], where a donor and acceptor semiconductor are combined - either in a simple planar stack [referred to as planar bi-layer heterojunction solar cell], or in an intermixed blend [referred to as bulk-heterojunction (BHJ) solar cell]. While the solar cell designed by Tang was in a planar device stack architecture [which generally suffers from low light harvesting efficiency leading to an overall low power conversion efficiency], the concept of blending an electron donating and accepting unit into a bulk-heterojunction was first applied to build a solar cell in 1991 by Hiramoto[24] and others[25].

On a macroscopic level, the working principle of an organic, excitonic solar cell contains multiple essential steps [26, 27] starting with (i) the absorption of a photon of energy $E_{\mathrm{ph}}$ in either the donor or acceptor unit of the bulk accompanied by an excitation of an electron from the [donor, or acceptor] HOMO to the LUMO followed by quick relaxation to the LUMO lowest energy state [as shown in step (i) in Fig. 1.3. This state is referred to as the singlet exciton state $\left[S_{1}\right]$. The exciton then diffuses to the donor: acceptor interface, shown in step (ii) in Fig. 1.3. When forming a staggered type-II heterojunction [as schematically shown in Fig. 1.3], the difference in HOMO and LUMO energy levels of donor and acceptor unit promote exciton dissociation at the 
donor: acceptor interface. 228] Here, electron [or hole] transfer from the donor [acceptor] LUMO [HOMO], $E_{\mathrm{D}, \mathrm{H}}\left[E_{\mathrm{A}, \mathrm{L}}\right]$, to acceptor [donor] LUMO [HOMO], $E_{\mathrm{A}, \mathrm{L}}\left[E_{\mathrm{D}, \mathrm{H}}\right]$, is energetically favoured [see step (iii) in Fig. 1.3. - commonly referred to as charge generation via channel 1 [2]. [29, 30]

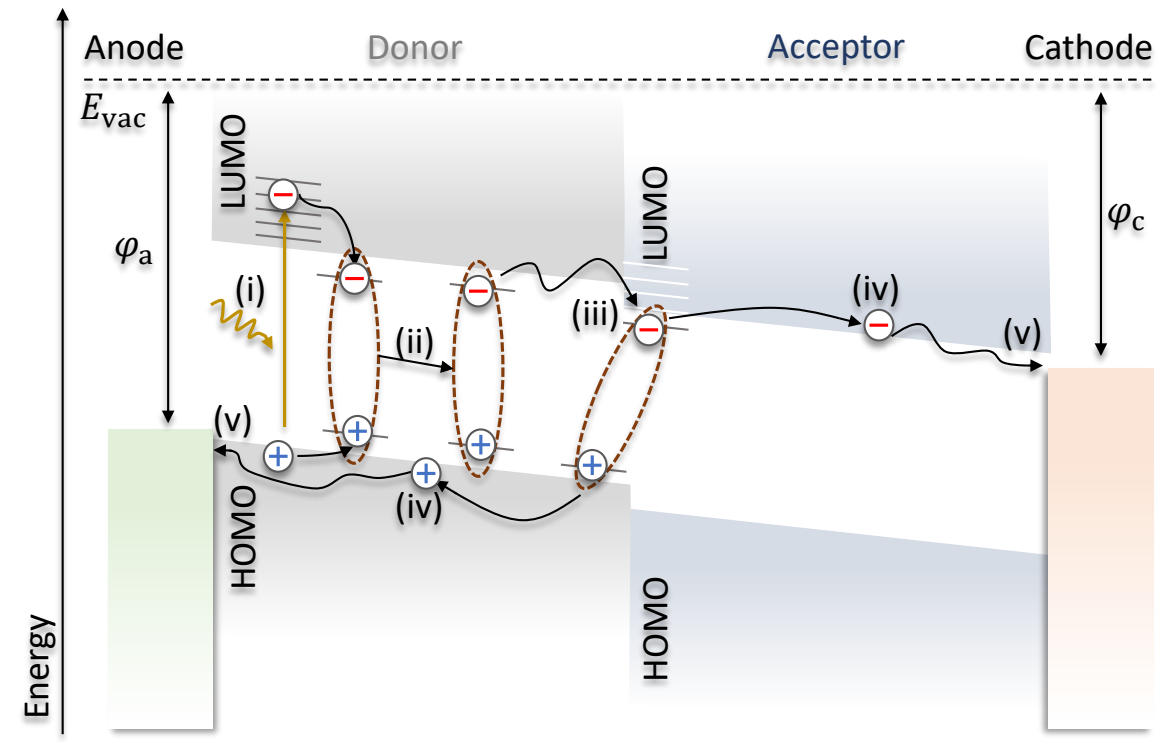

Figure 1.3: Schematic, simplified energy diagram of a donor: acceptor solar cell. Once a photon is absorbed in the donor material [i.e., when the energy of the photon is equal, or larger than the optical gap of the donor material], an electron is excited from the ground state to an [vibronically] excited states, and a Coulombically bound electron-hole pair [exciton] is formed. The exciton diffuses to the donor: acceptor interface. After chargetransfer from the donor LUMO to the acceptor LUMO, a charge transfer [CT] state is formed. The Coulombically bound CT state, energetically determined by the difference in donor HOMO and acceptor LUMO, is lower compared to the singlet excited and charge separated [CS] state. Prior to charge extraction at the electrodes, the free and separated electrons and hole are transported to the anode and cathode after CT state dissociation.

The excitation of this inter-molecular state formed at the interface [referred to as charge transfer (CT) state, where the excited electron in the acceptor LUMO is Coulombically bound to the hole in the donor HOMO] is energetically lower compared to donor and acceptor intra-molecular $S_{1}$ states, respectively. The energetic difference between $S_{1}$ and CT state, $\Delta E=E_{S_{1}}-E_{\mathrm{CT}}$, is often referred to as driving force for charge generation [or exciton dissociation]. While in most fullerene-acceptor based organic solar cells, $\Delta E$ is found to be a few hundred milli electronvolt [31, 32], very low driving forces $[\Delta E \rightarrow 0]$ are observed for non-fullerene acceptor based organic solar cells. [22, 33] It is 


\section{THEORETICAL BACKGROUND AND FUNDAMENTALS}

believed that the high crystallinity, low degree of [energetic] disorder, favourable optical gaps [i.e., narrower to the corresponding semiconducting donors), and long exciton lifetimes [respectively, long diffusion lengths], are the reasons for low driving forces, but yet efficient exciton-to-CT, and CT-to-charge separated [CS] state transitions in non-fullerene acceptor-based organic solar cells. [22] Once the CT state is dissociated and charge carrier separation is achieved [see step (iv) in Fig. 1.3], the free and mobile electron and hole are transported to the electrodes [driven by an internal electric field $E_{\text {elec }}$, generated by the difference in work-function of anode, $\varphi_{\mathrm{a}}$, and cathode, $\varphi_{\mathrm{c}}$ ], where they can be finally extracted [see step (v) in Fig. 1.3.

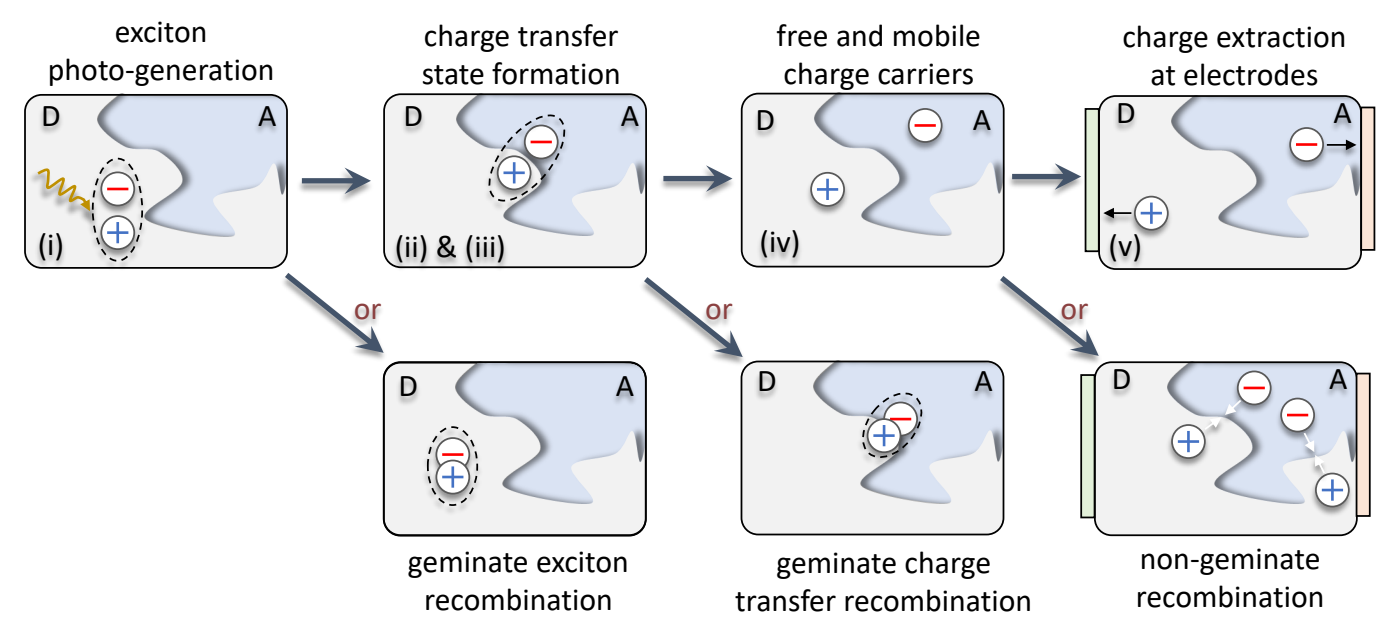

Figure 1.4: After photo-generation of an exciton in the donor (i), the exciton diffuses to the donor: acceptor interface (ii), where a charge transfer [CT] state is formed (iii). Once the CT state is dissociated (iv), the free and mobile charge carriers are transported to the electrodes, where the electrons and holes can be extracted (v). Different recombination pathways act as charge carrier loss channels. As such, the photo-generated exciton and the interfacial CT state can [geminately] recombine/decay back to the ground state, or, separated charge carriers can non-geminately recombine.

The presence of high energy barriers [e.g., induced through non-ohmic and non-selective contacts at the electrode/bulk interface, or non-favourable donor and acceptor HOMO and LUMO energy levels] can lead to a loss of photo-generated electrons and holes most prominently through recombination. As depicted in Fig. 1.4, recombination of charge carriers in the photoactive layer can occur geminately in case of excitons and CT states [referred to as geminate, or initial recombination], or non-geminately between separated and mobile carriers generated independently of each other [referred to 
as non-geminate recombination]. These recombination processes not only take place at different locations within the device [e.g., exciton recombination in single donor (or acceptor) domains; CT state recombination at donor/acceptor interfaces; surface recombination at bulk/electrode interfaces], but also cover a broad time window from pico-seconds [ps], typical of exciton recombination, to micro-seconds [ $\mu \mathrm{s}]$, typical of bulk recombination.

The overall efficiency $\eta_{\text {overall }}$ [or, external quantum efficiency, EQE] of any [excitonic] solar cell depends on the optoelectronic properties of semiconducting [i.e., donor and acceptor] and extracting [i.e., anode and cathode] components [including thin-film morphology], and hence on the single efficiencies of all processes described above, namely photon absorption $\left[\eta_{\text {abs }}\right]$, exciton diffusion $\left[\eta_{\mathrm{E}, \mathrm{diff}}\right]$ and dissociation $\left[\eta_{\mathrm{E}, \mathrm{diss}}\right]$, CT state dissociation $\left[\eta_{\mathrm{CT}, \mathrm{diss}}\right]$ as well as charge transport and collection $\left[\eta_{\text {col }}\right]$. Hence, the overall efficiency of an organic solar cell can be expressed as:

$$
\eta_{\text {overall }}=\eta_{\mathrm{abs}} \eta_{\mathrm{E}, \mathrm{diff}} \eta_{\mathrm{E}, \mathrm{diss}} \eta_{\mathrm{CT}, \mathrm{diss}} \eta_{\mathrm{col}}
$$

It should be noted that only the latter two efficiencies depend on the electric field $E_{\text {elec}}$, while all are influenced by temperature. 26] Thin-film morphology, in turn, has a non-negligible impact on exciton diffusion, CT state dissociation and charge transport/collection efficiency. As such, only excitons that reach the donor: acceptor interface within their limited exciton lifetime $\left[\tau_{\text {exc }}\right]$ can dissociate. In other words, exciton dissociation occurs when the exciton diffusion length $L_{\mathrm{D}}=\sqrt{D \tau_{\mathrm{exc}}}$ [where $D$ is the exciton diffusion coefficient] is larger than the [average] distance between exciton creation and donor: acceptor interface; typically, a few tens of nanometers. 34-36] The domain size of donor and acceptor units and their degree of intermixing determines, and thus limits, the amount of available interfaces and CT states.[37, 38] In a similar manner, thin-film morphology is responsible for the availability of continuous, singledomain pathways required for separated charges [subsequent to CT state dissociation] to reach the electrodes. [39] The thickness $d$ of organic photoactive layers commonly varies between tens and hundreds of nanometers [40], and thus is on the order of ultraviolet $[d<300 \mathrm{~nm}$ ], visible [or VIS; $350 \mathrm{~nm}<d<800 \mathrm{~nm}$ ] and infrared [or IR; $d>800 \mathrm{~nm}$ ] wavelength regions. Subsequently, low-finesse cavity-effects - such as the 


\section{THEORETICAL BACKGROUND AND FUNDAMENTALS}

formation of standing waves inside the active layer of the photovoltaic device due to interference between incident and electrode back-reflected light - need to be considered when probing optoelectronic devices with photo-physical experiments. [41- 43 ]

Based upon Eq. 1.12, different parameters can be defined and measured which are commonly used in the field of organic photovoltaic [including solar cells, and photodetectors] to characterize and evaluate physical processes governing those devices. As such, the external [internal] quantum efficiency EQE [IQE] [defined as the ratio of extracted charge carriers to incoming photons; $\mathrm{EQE}=\eta_{\mathrm{abs}} \eta_{\mathrm{E}, \mathrm{diff}} \eta_{\mathrm{E}, \mathrm{diss}} \eta_{\mathrm{CT} \text {,diss }} \eta_{\mathrm{col}}=$ $\left.\eta_{\text {abs }} \mathrm{IQE}\right]$, the external [internal] generation efficiency EGE [IGE] [defined as the ratio of generated charge carriers to incoming photons; EGE $=\eta_{\mathrm{abs}} \eta_{\mathrm{E}, \mathrm{diff}} \eta_{\mathrm{E} \text {,diss }} \eta_{\mathrm{CT} \text {,diss }}=$ $\left.\eta_{\mathrm{abs}} \mathrm{IGE}\right]$, and the charge generation quantum yield [CGY] [defined as the probability to generate free carriers] have been, among others, established as reliable quantities. Throughout the history of photovoltaic, different steady-state and transiente measurement techniques have been developed, each of which seeks to probe, inter alia, one of above quantities, improve the accuracy of their determination, simplify an existing measurement setup, and/or extend their applicability. 


\subsection{Basic device characterization}

Ohmic and selective contacts allow [in the ideal case] only one type of charge carrier to be injected, while the opposite is extracted. Hence, to drive a current through a solar cell [which can, in the simplest picture, be considered as a p-n diode] in dark, the injected electrons and holes are required to recombine - an event whose probability depends on both electron $[n]$ and hole $[p]$ carrier density. Based upon the mass action law [see Section 1.2], the recombination probability [linked to the product $n p$ ] and the current $i$ are related to one another via $i \propto n p \propto \exp \left(-E_{\mathrm{g}}\left[k_{\mathrm{B}} T\right]^{-1}\right)$ [see Eq. 1.9. By applying a forward bias voltage $V$, the energy barrier can be lowered by $q V$, so that $\exp \left(-E_{\mathrm{g}}\left[k_{\mathrm{B}} T\right]^{-1}\right)$ becomes $\exp \left(\left[q V-E_{\mathrm{g}}\right]\left[k_{\mathrm{B}} T\right]^{-1}\right)$, hence $i \propto \exp \left(q V\left[k_{\mathrm{B}} T\right]^{-1}\right)$. This exponential relation between current and energy barrier is, in slightly modified form, referred to as Shockley diode equation. 44 Even though William Shockley derived this relation for inorganic diodes, the Shockley diode equation has been successfully adapted to organic p-n diode-based applications, such as organic solar cells. 45.

From an electrical point of view, a solar cell can be considered as a diode which is in parallel with (i) a shunt resistance $\left[R_{\mathrm{p}}\right]$ accounting for leakage currents, (ii) an external current source $\left[j_{\mathrm{ph}}\right]$ supplying a certain photocurrent density, and (iii) in series with a resistance $\left[R_{\mathrm{s}}\right]$ to account for contact resistance. Hence, the current density [defined as the current normalized to the area $A$ of the active layer; $j=i[A]^{-1}$, typically in the unit of $\mathrm{mA} \mathrm{cm}^{-2}$ ] versus voltage characteristics of a solar cell can be described by an extended Shockley diode equation taking both series and shunt resistances into account: 46

$$
j=j_{0}\left\{\exp \left[\frac{q\left(V-j R_{\mathrm{s}}\right)}{n_{\mathrm{id}} k_{\mathrm{B}} T}\right]-1\right\}+\frac{V-j R_{\mathrm{s}}}{R_{\mathrm{p}}}-j_{\mathrm{ph}}
$$

Here, $n_{\text {id }}$ denotes the ideality factor representing the predominant recombination mechanism, and takes values between 1 and 2. 44 -49] Fig. 1.5 a shows the current density versus applied voltage $(J-V)$ characteristic of an organic solar cell under illumination [solid green line] [i.e., artificial 1 sun light under air mass [AM] 1.5G condition] and in dark [dashed green line] identifying several characteristic marks. At voltage $V_{\text {oc }}$ [referred to as open-circuit voltage], the net current density is zero. The $V_{\text {oc }}$ can be 


\section{THEORETICAL BACKGROUND AND FUNDAMENTALS}

explicitly expressed as a function of device parameters such as the ideality factor, the photocurrent density, and the dark saturation current density $\left[j_{0}\right]:[50]$

$$
V_{\mathrm{oc}}=\frac{n_{\mathrm{id}} k_{\mathrm{B}} T}{q} \ln \left[\frac{j_{\mathrm{ph}}}{j_{0}}+1\right]
$$

Assuming [bimolecular] recombination [i.e., $n_{\text {id }}=1$ ] of free charge carriers taking place via $\mathrm{CT}$ states to be dominant, one can alternatively express the $V_{\mathrm{oc}}$ as a function of active layer parameters, such as the $\mathrm{CT}$ state energy $\left[E_{\mathrm{CT}}\right]$, the density of available $\mathrm{CT}$ states $\left[N_{\mathrm{CT}}\right]$, the $\mathrm{CT}$ recombination rate $\left[k_{\mathrm{f}}\right]$, and the generation rate $[G]:[51]$

$$
V_{\mathrm{oc}}=\frac{E_{\mathrm{CT}}}{q}-\frac{k_{\mathrm{B}} T}{q} \ln \left[\frac{G}{k_{\mathrm{f}} N_{\mathrm{CT}}}\right] \text {. }
$$

At zero applied voltage, the current density $j_{\mathrm{sc}}$ [referred to as short-circuit current density] flowing through the solar cell corresponds to the photocurrent density generated by the solar cell under the condition of no external load on the cell [referred to as shortcircuit conditions]. The device photocurrent density generated upon illumination, $j_{\mathrm{ph}}$, can be determined from the [wavelength dependent] EQE spectrum of a photovoltaic device, and the spectral flux $\varphi$ of the light source [see Fig. 1.5 b]:

$$
j_{\mathrm{ph}}=q \int_{E_{0}}^{\infty} \operatorname{EQE}(E) \varphi(E) d E
$$

where $E_{0}$ is the photon energy lower limit to the integral. It is worth noting that the photocurrent density depends on incident light intensity [i.e., charge carrier density], the mobility of electrons and holes in the photoactive layer, the presence of sub-gap trap states in the donor: acceptor bulk, and other morphological and electro-optical properties of the solar cell. For applied voltages between zero and $V_{\mathrm{oc}}$, the solar cell generates power [red solid line in Fig. 1.5 a], which is maximized at the so-called maximum power point characterized by a corresponding maximum power point voltage $\left[V_{\mathrm{MPP}}\right]$ and current density [ $\left.j_{\mathrm{MPP}}\right]$, and defining the solar cell operating point. For $V<0$ and $V>V_{\text {oc }}$, in turn, the device consumes power, thus does not operate as a solar cell anymore. The ratio of the product of $V_{\mathrm{MPP}}$ and $j_{\mathrm{MPP}}$ to the product of $V_{\mathrm{oc}}$ and $j_{\text {sc }}$ is defined as the so-called fill factor [FF] [green shaded square in Fig. 1.5a]: 46]

$$
\mathrm{FF}=\frac{V_{\mathrm{MPP}} j_{\mathrm{MPP}}}{V_{\mathrm{oc}} j_{\mathrm{sc}}}
$$


which is used to determine the overall power conversion efficiency $[\mathrm{PCE}]$ :

$$
\mathrm{PCE}=\frac{V_{\mathrm{oc}} j_{\mathrm{sc}} \times \mathrm{FF}}{P_{\mathrm{in}}}=\frac{P_{\mathrm{out}}}{P_{\mathrm{in}}} .
$$

Here, $P_{\text {out }}$ is the power output from the solar cell and $P_{\text {in }}$ is the power of the incident photon-flux.

The $J-V$ curve under illumination of a photovoltaic device can be used to identify basic power loss channels, which can be generally categorized as intrinsic and extrinsic losses. As schematically shown in Fig. 1.5c, intrinsic, and hence often unavoidable, losses include (i) the spectral mismatch between the emitted solar spectrum and the photovoltaic device bandgap-limited absorption spectrum [i.e., photons with $E_{\mathrm{ph}}<E_{\mathrm{g}}$ are not absorbed], (ii) a thermalization loss due to vibrational interactions of excited charge carriers with phonons in case that $E_{\mathrm{ph}}>E_{\mathrm{g}}$, (iii) the emission loss of a photovoltaic device itself [referred to as Kirchoff's law, which states that an absorber is also an emitter], (iv) thermodynamic losses due to the energy-demanding conversion process of thermal energy into electric power [often referred to as the Carnot cycle], and (v) entropy generation loss due to a solid angle mismatch in absorption and emission of the photovoltaic device. [52] The ensemble of all intrinsic losses defines the theoretical performance limit of a solar cell [referred to as Shockley-Queisser (SQ) limit, see Fig. 1.5c] which is, in case of a single-junction [inorganic] system, $\sim 33 \%$, and was first determined by Shockley and Queisser in 1961 for inorganic p-n junctions. [53]

It is worth noting that the SQ-limit can be varied, inter alia, through combining different active layers with absorption in complementary solar spectrum regions to maximize the light harvesting efficiency [commonly referred to as tandem devices, while solar cells with active layers composed of three semiconductors, in particular, are referred to as ternary solar cells], or by changing the incident concentration of solar spectrum absorbed by the photovoltaic device [e.g., in so-called light-concentrator solar cells]. 


\section{THEORETICAL BACKGROUND AND FUNDAMENTALS}
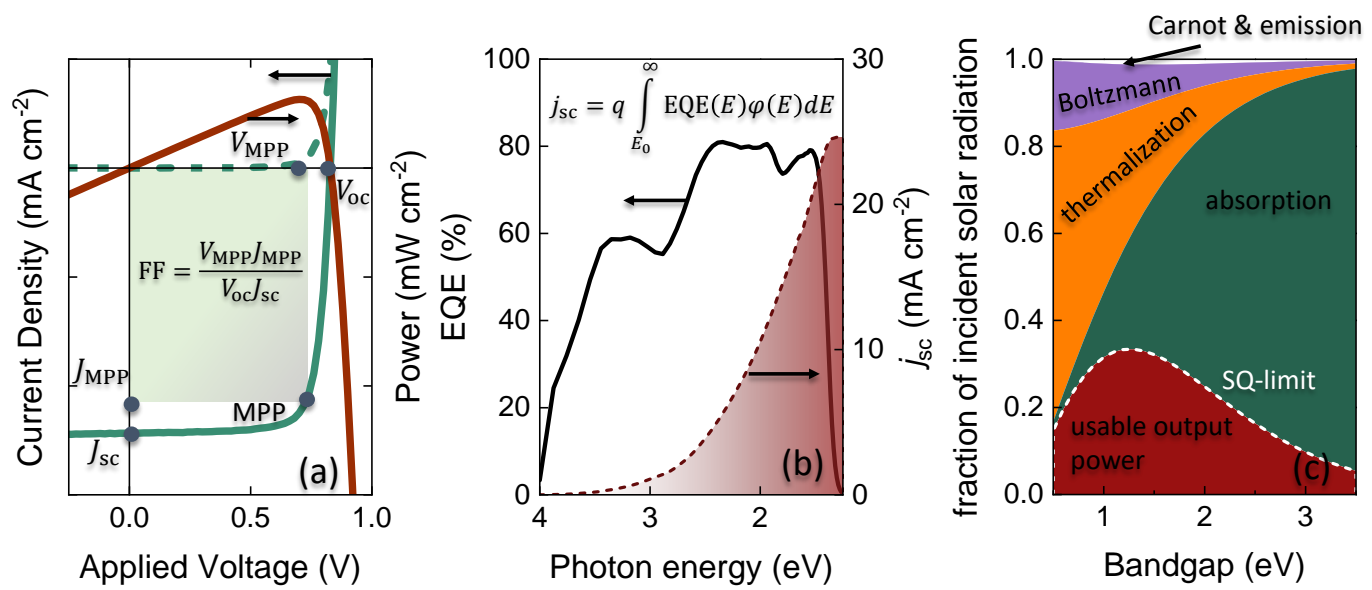

Figure 1.5: (a) Schematic current density versus applied voltage curve in the dark [green dashed line] and under artificial sun light [AM 1.5G condition] [green solid line], and compared with the output power [red line]. Basic parameters are marked: short-circuit current density $\left[j_{\mathrm{sc}}\right]$, open-circuit voltage $\left[V_{\mathrm{oc}}\right]$, maximum power point current density $\left[j_{\mathrm{MPP}}\right]$, maximum power point voltage $\left[V_{\mathrm{MPP}}\right]$, and maximum power point $[\mathrm{MPP}]$. The green shaded square illustrates the fill factor $[\mathrm{FF}]$ of the solar cell. (b) External quantum efficiency [EQE] spectrum [left axis] and calculated integrated photocurrent density, $j_{\mathrm{ph}}$, [right axis] of a solar cell plotted as a function wavelength. The maximum photocurrent density corresponds to the upper limit of the integral of EQE and solar flux spectrum product. (c) Schematic representation of the bandgap-dependent intrinsic losses in solar cells calculated along [52]. The Shockley-Queisser [SQ] limit is displayed for comparison.

Due to the excitonic and disordered nature of organic semiconductors it is assumed that the efficiency limit for [single-junction] organic solar cells is approximately 25 $\%[22,54]$ and hence slightly lower compared to their inorganic single-junction counterparts. Extrinsic losses, in turn, come from inefficient generation, transport, and extraction processes. As such, low photocurrents and photovoltages in light $J$ - $V$ curves of organic solar cells are typically expressions of poor charge transport [i.e., $\eta_{\text {col }}<1$, see Eq. 1.12], non-ideal bulk and interface energetics [i.e., $\eta_{\mathrm{E}, \mathrm{diff}}, \eta_{\mathrm{E}, \mathrm{diss}}, \eta_{\mathrm{CT}, \mathrm{diss}}, \eta_{\mathrm{col}}<1$, see Eq. 1.12], and non-optimized morphology [i.e., $\eta_{\mathrm{E}, \mathrm{diff}}, \eta_{\mathrm{CT}, \mathrm{diss}}, \eta_{\mathrm{col}}<1$, see Eq. 1.12. As such, large donor: acceptor domains [e.g., due to high molecular weight [55]], large energy gaps [e.g., due to a spectral absorption mismatch between donor and acceptor units], and various photocurrent loss mechanisms [e.g., trap-assisted recombination, build-up of space-charge, bimolecular recombination, series resistance limitations, etc.] can result in small photocurrents. Non-radiative recombination [i.e., the interaction of vibrational and electronic states in the bulk], surface recombination [56] [i.e., 
the extraction of minority carriers at the 'wrong' electrode], and small donor: acceptor bandgaps [57] can, in turn, lead to low open-circuit voltages or high photovoltage losses. All the above mentioned sources of photocurrent and photovoltage losses are direct consequence of non-optimized device architecture and inefficient physical processes [see Eq. 1.12], e.g., the transport and collection of electrons and holes, or the generation and recombination of charge carriers in the donor: acceptor bulk] governing the photovoltaic device. A precise and careful examination of the fundamental charge processes is thereby essential for a correct identification of carrier loss channels. Based on this understanding device fabrication can be optimized and higher device efficiency can be achieved.

The following sections will draw the fundamental concepts of charge generation and recombination processes in thin-film optoelectronic devices and thereby chronologically link $\eta_{\mathrm{E}, \text { diff, }} \eta_{\mathrm{E}, \text { diss }}, \eta_{\mathrm{CT} \text {,diss }}$ and $\eta_{\mathrm{col}}$ to basic, electro-optical properties of organic, photovoltaic devices. 


\section{THEORETICAL BACKGROUND AND FUNDAMENTALS}

\subsection{Charge generation and geminate recombination}

In excitonic materials, charge generation often refers to a combination of mechanisms, based on which different electronic states can be defined [see Fig. 1.6a]. 27, 58], most importantly singlet excited states [or, ES], and intermediate and Coulombicallybound CT states. As mentioned in Section 1.5, the latter ones are energetically lower compared to the donor and acceptor ES - most prominently seen in photovoltaic external quantum efficiency [EQE $\mathrm{PV}]$ and electroluminescence [EL] experiments. [49, 59, 60] While in $\mathrm{EQE}_{\mathrm{PV}}$, the $\mathrm{CT}$ state is populated directly through photon absorption, EL refers to the case of indirect $\mathrm{CT}$ state population via injected charge recombination at the donor: acceptor interface.
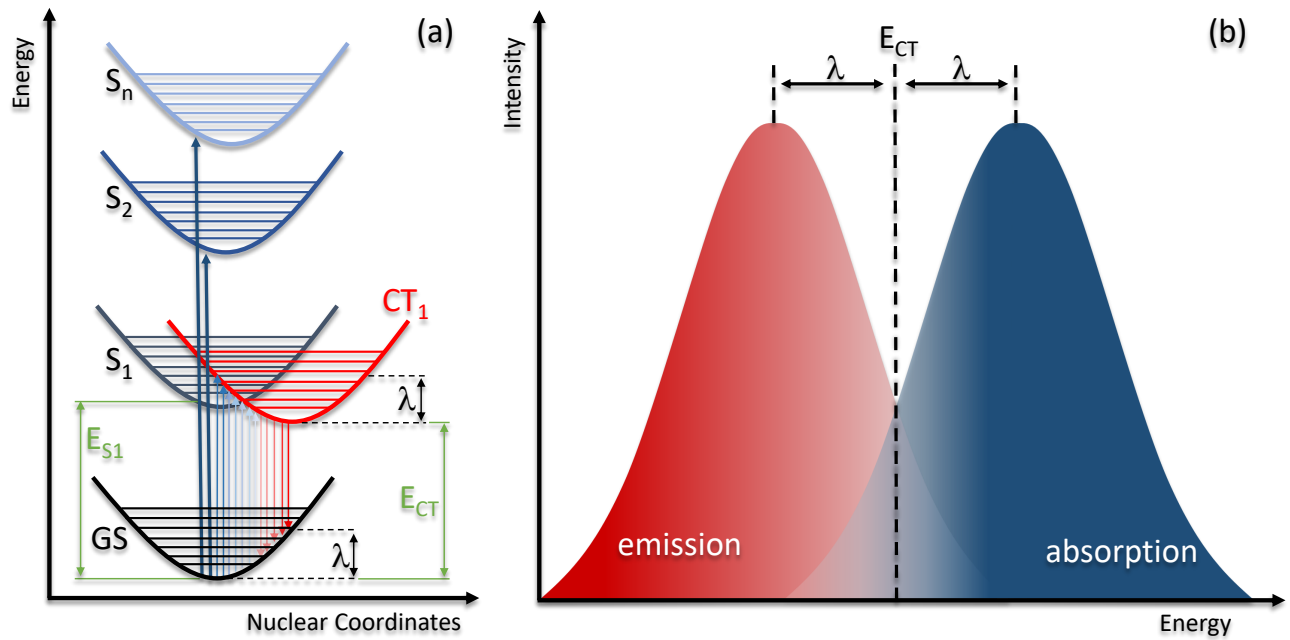

Figure 1.6: (a) Electronic states involved in the charge generation process. After absorption of a photon, an electron-hole pair is excited in either the donor or acceptor molecule from the ground state (GS) to an excited state $\left(S_{1}, S_{2}, \ldots, S_{\mathrm{n}}\right)$. Blue arrows depict optical transitions (i.e., absorption). The red vertical arrows depict the optical transition (i.e., emission) from the CT state back to the GS. (b) Emission (red) and absorption (blue) spectra for optical transitions between GS and CT state. $\lambda$ is the reorganization energy, while $E_{\mathrm{CT}}$ is the energy of the charge transfer state.

Inter- and intra-molecular vibrations [i.e., phonons] in a single molecule, and between multiple conjugated systems, lead to [low- and high-frequency] sub-levels [typically referred to as vibronic modes] in electronic states [see Fig. 1.6a]. The contribution of such vibronic modes on optical transitions is commonly expressed in the reorganisation energy $[\lambda]$ of a system. [51] Given that the electronic states in organic semi- 
conductors are populated following Boltzmann statistics, optical transitions [that are, photon-absorption and radiative decay] between ground state [GS] and excited states are homogeneously broadened rather than featured by discrete peaks as one would expect based upon the Frank-Condon principle [see Fig. 1.6b]. Here, the emitted photon flux $\left[\varphi_{\mathrm{ph}, \mathrm{emitted}}(E)\right]$ and absorption $[A(E)]$ spectrum are related to one another via $\varphi_{\text {ph,emitted }}(E) \propto A(E) E^{2} \exp \left\{-E\left[k_{\mathrm{B}} T\right]^{-1}\right\}$. [51, 61] It is worth noting that the broadening of GS-to-ES and ES-to-GS optical transitions are also induced by intrinsic variations in molecular conformations, and conjugated system packing of adjacent molecules in donor: acceptor blends, referred to as static disorder. [62] 


\section{THEORETICAL BACKGROUND AND FUNDAMENTALS}

\subsubsection{From excitons to charge transfer states}

Based upon the average distance between the excited electron and the remaining hole, that is, the exciton-radius, two types of excitons are distinguished: Frenkel-type and Wannier-Mott-type. While the former one typically refers to as excitons in disordered, low dielectric constant [i.e., organic] semiconductors having strong Coulomb binding energies [typically several hundreds of milli electronvolt [63]] and hence, an exciton-radius comparable to a single molecular unit, the latter one refers to weakly-bound excitons [typically a few tens of milli electronvolt] in crystalline, ordered, and high dielectric constant materials having an exciton-radius larger than the lattice constant.

Semiconductors with low enough binding energy [e.g., where $E_{\mathrm{b}}$ is smaller than thermal energy at room temperature] spontaneously separate excitons into unbound electrons and holes and are therefore classified as non-excitonic [ordinarily inorganic semiconductors, such as $\mathrm{Si}$, and GaAs]. With increasing binding energy, the separation of excitons at room temperature becomes less likely, and the electronic properties are dominated by the excitons - semiconductors that exhibit this behaviour are classified as excitonic [typically organic semiconductors]. Once an exciton is created, it can recombine [in case of Frenkel-type], relax back to the ground state via lattice phonon interaction [in case of Wannier-Mott-type], or diffuse towards a lower exciton concentration part of the bulk. Hence, exciton recombination and relaxation to the GS lead to $\eta_{\mathrm{E} \text {,diff }}<1$. Only if the exciton reaches the donor: acceptor interface, it can form a charge transfer state - the precursor step for free charge formation [see Section 1.5. The thermally activated process of exciton dissociation is commonly described as a charge transfer process obeying Marcus theory. 64 ] 


\subsubsection{From charge transfer states to free carriers}

For the case that the exciton diffuses to the donor: acceptor interface, it relaxes to a lower-energy, Coulombically-bound CT state [or, charge-transfer exciton]. As mentioned before, excited states [including excited charges, excitons, and CT states] can relax to the lowest, excited state via so-called thermalization [defined as an energy transfer through phonon emission, or hopping to a lower lying site in an (energetically) disordered conjugated system]. The role of excess energy and thermalization on the generation pathway to free charge carriers is still subject of current debate. On the one hand, CT state dissociation is found to proceed through excited CT states [often referred to as hot charge transfer states] generated by high energy excitons and thus, depending on the excess energy of the $E_{S_{1}}$ state. [65] On the other hand, studies have reported charge generation in polymer: fullerene BHJ blends to be independent of excess energy. [61, 66] By conducting time delayed collection field [TDCF] experiments, Kurpiers et al. found [electric field and temperature dependent] charge generation to proceed through thermalized and relaxed CT states - independent of the energetic offset between $\mathrm{CT}$ and $S_{1}$ states [referred to as driving force for exciton dissociation]. [66] It is assumed that this energetic offset is a requirement for efficient charge generation, an assumption that has recently been challenged by the emergence of the non-fullerene acceptors. 22. In this regard, Perdigón-Toro et al. reported a barrierless free charge generation in state-of-the-art high-efficiency PM6:Y6 thin-film solar cells. [33]

The first description of the dissociation/recombination of interfacial CT states was based upon a work by Onsager et al. in 1938. In this work the description of the generation of free charges in a weak electrolyte was based upon the Brownian diffusioninduced motion and the assumption that the CT state electron and hole have an initial distance $r_{0} \cdot[67$ Onsager's models assumed a temperature-activated charge generation with an activation energy given by the Coulomb attraction [of the electron/hole pair at $r_{0}$ ]. However, while it could correctly describe the relation between CT state dissociation and applied electric field in few organic semiconductor systems, the model failed when systems exhibited electric-field independent dissociation. In 1984, Onsager's model was revised and extended by Braun through a kinetic approach involving the consideration of CT state lifetimes $\tau$. [68] Herein, CT states are assumed to either recombine geminately to the ground state [with the rate constant $k_{\mathrm{f}}$ ] or dissociate 


\section{THEORETICAL BACKGROUND AND FUNDAMENTALS}

into free carriers [with the rate constant $k_{\mathrm{d}}$ ]. The efficiency $\eta_{\mathrm{CT} \text {,diss }}$ for a CT state to dissociate into free carriers can then be expressed as: 68 ]

$$
\eta_{\mathrm{CT}, \mathrm{diss}}(E)=\frac{k_{\mathrm{d}}(E)}{k_{\mathrm{d}}(E)+k_{\mathrm{f}}}=k_{\mathrm{d}}(E) \tau_{\text {total }}(E)
$$

where $\tau_{\text {total }}=\left[k_{\mathrm{d}}(E)+k_{\mathrm{f}}\right]^{-1}$ defines the total CT state lifetime. It should be noted that, other than the dissociation component, the geminate recombination rate is assumed to be independent of the electric field.
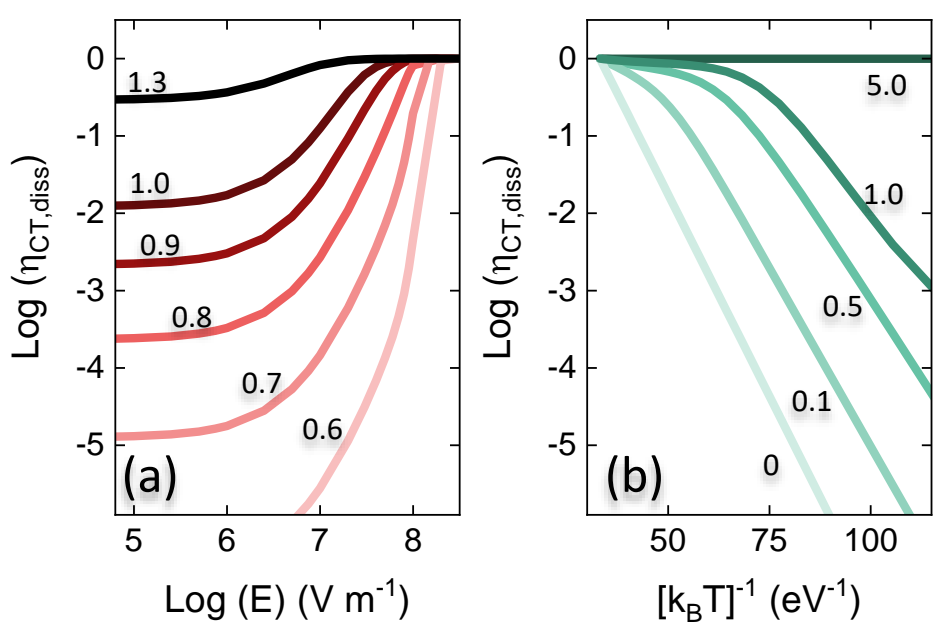

Figure 1.7: (a) Dissociation probability calculated in accordance with the Onsager-Braun model, and plotted as a function of electric field. The electron-hole distance was varied between $1.3 \mathrm{~nm}$ and $0.6 \mathrm{~nm}$, while a dielectric constant of $\varepsilon=3$ and a carrier mobility of $\mu=10^{-4} \mathrm{~cm}^{2} \mathrm{~V}^{-1} \mathrm{~s}^{-1}$ were assumed. The curves are labelled with the value of electronhole distance in $[\mathrm{nm}]$. (b) Dissociation probability plotted as a function of inverse thermal energy. The electron-hole distance was set to $2 \mathrm{~nm}$, and $\varepsilon=3$ and $\mu=10^{-4} \mathrm{~cm}^{2} \mathrm{~V}^{-1} \mathrm{~s}^{-1}$ were assumed. The curves are labelled with the assumed electric field $\left[i n 10^{7} \mathrm{Vm}^{-1}\right]$.

Braun assumed that during one lifetime, a CT state can partially dissociate and recombine [with a bimolecular recombination rate constant $k_{\mathrm{r}}=e\left(\mu_{\mathrm{h}}+\mu_{\mathrm{e}}\right)\left[\varepsilon \varepsilon_{0}\right]^{-1}$, in accordance with Langevin theory, see Section 1.8.1 below] and subsequently re-generate CT states. This is in contrast with Onsager's theory, where recombined species are not further considered. In Braun's model, the temperature and electric field dependent dissociation rate can be expressed as: 68 .

$$
k_{\mathrm{d}}=\frac{3 \mu e}{4 \pi \varepsilon \varepsilon_{0} a^{3}} \times \exp \left[-\frac{\Delta E}{k_{\mathrm{B}} T}\right] \times\left[1+b+\frac{b^{2}}{2}+\frac{b^{3}}{18}+\ldots\right],
$$


where $a$ denotes the ion pair radius, $b=e^{3} E\left[8 \pi \varepsilon \varepsilon_{0}\left(k_{\mathrm{B}} T\right)^{2}\right]^{-1}$, and $\Delta E=e^{2}\left[4 \pi \varepsilon \varepsilon_{0} a\right]^{-1}$ is the free carrier dissociation [energy] barrier [i.e., the CT state binding energy] in the case of ion pair assumption. Following $k_{\mathrm{d}}(E, T)$, the CT state dissociation probability also depends on the temperature and the electric field [see Fig. 1.7]. As shown in Fig. 1.7 a, efficient charge generation is [assuming typical device parameters, such as $d=100 \mathrm{~nm}, \varepsilon=3, \mu=10^{-4} \mathrm{~cm}^{2} \mathrm{~V}^{-1} \mathrm{~s}^{-1}, T=300 \mathrm{~K}$, and a built-in voltage of $V_{\mathrm{bi}}=1$ $\mathrm{V}]$ expected to occur at electric fields $E_{\text {elec }}=\left|V_{\mathrm{appl}}-V_{\mathrm{bi}}\right| d^{-1}$ as large as $10^{7} \mathrm{Vm}^{-1}$ [which corresponds to $\sim 0 \mathrm{~V}$ applied bias voltage]. Hence, for most neat, organic solar cells, inefficient CT state dissociation is expected under open-circuit conditions [i.e., when the internal electric field approaches zero, corresponding to applied voltage of $\left.\sim V_{\mathrm{bi}}\right]$. 


\section{THEORETICAL BACKGROUND AND FUNDAMENTALS}

\subsection{Charge collection and non-geminate recombination}

As discussed above, small, conjugated molecular units in disordered, low-mobility semiconducting materials accompanied by energetic and spatial disorder make the bandtransport model [which assumes delocalized charges as it is the case in ordered, crystalline semiconductors] not applicable for organic semiconductors [see Fig. 1.2. Instead, a hopping-transport model is used in organic semiconductors, in which charge transport is described as a hopping process [often referred to as thermally activated tunnelling] of charge carriers between differently aligned, adjacent molecule sites, as formulated in the Miller-Abraham theory: 69 ]

$$
k_{\mathrm{ij}}=\nu_{0} \exp \left[-\gamma r_{\mathrm{ij}}\right] \begin{cases}\exp \left[-\frac{E_{\mathrm{j}}-E_{\mathrm{i}}}{k_{\mathrm{B}} T}\right] & \text {,if } E_{\mathrm{j}}>E_{\mathrm{i}} \\ 1 & \text {,if } E_{\mathrm{j}} \leq E_{\mathrm{i}} .\end{cases}
$$

Here, the hopping rate $k_{\mathrm{ij}}$ depends on the maximum hopping rate $\nu_{0}$, the distance $r_{\mathrm{ij}}$ between two sites $i$ [with energy $E_{\mathrm{i}}$ ] and $j$ [with energy $E_{\mathrm{j}}$ ] [as schematically shown in Fig. 1.2 c] , and the inverse localization radius $\gamma$ [related to how well a tunnel process of charges from site $i$ to $j$ is]. It becomes clear that while the first exponential term in Eq. 1.21 is related to the tunnelling process, the second exponential term in Eq. 1.21 accounts for the thermal contribution to hopping. Furthermore, it is important to note that the hopping rate depends on the energy of the available states [i.e., $E_{\mathrm{i}}$ and $E_{\mathrm{j}}$ ], hence on the distribution reflected in the density of states, DOS. Hopping processes with $E_{\mathrm{j}}>E_{\mathrm{i}}\left[E_{\mathrm{j}} \leq E_{\mathrm{i}}\right]$ are defined as thermally activated upward [independent downward] hops. The hopping velocity $v_{\mathrm{ij}}$ is proportional to the hopping rate and can be expressed as $v_{\mathrm{ij}}=r_{\mathrm{ij}} k_{\mathrm{ij}}=r_{\mathrm{ij}} t_{\mathrm{ij}}^{-1}$, where $t_{\mathrm{ij}}$ denotes the required time for a single hop of a charge from one to another site. The mobility of charge carriers $\mu$ is then given by $\mu=v_{\mathrm{ij}} E_{\text {elec }}^{-1}$, and defines the time $t_{\mathrm{tr}}=d^{2} \mu^{-1} \mid U^{-1}$, photogenerated charge carriers need to be extracted at the electrodes [or alternatively, the time to transit the distance $d$ from one to another electrode, $\left.\tau_{\mathrm{tr}}\right]$ under the influence of a potential $U=V-V_{\mathrm{bi}}$. The temperature dependence of hopping rate and DOS are reflected in the charge carrier mobility via $\mu(T)=\mu_{0} \exp \left\{-\left[\frac{2 \sigma}{3 k_{\mathrm{B}} T}\right]^{2}\right\}$, where $\sigma$ denotes the energetic disorder. [70] The spatial distribution of hopping sites [often referred to as spatial disorder] is typically described via an electric field dependent mobility $\mu\left(E_{\text {elec }}\right) \sim \exp \left(\beta_{\text {disorder }} \times \sqrt{E_{\text {elec }}}\right)$ 
$\left[\beta_{\text {disorder }}\right.$ denotes a pre-factor which is related to the spatial disorder $\left.\Pi\right]$. Given the finite lifetime $\tau$ of excited and separated charge carriers [referring to carriers after CT dissociation], one needs to consider non-geminate recombination when evaluating charge transport processes in optoelectronic devices. Different types of recombination are distinguished based upon their reaction-order, which states how many reactants are involved into a [non-geminate, or geminate] recombination event. In particular, non-geminate recombination processes [taking place after CT state dissociation] will directly influence the charge transport of extractable charge carriers, hence will lead to $\eta_{\mathrm{col}}<1$. 


\section{THEORETICAL BACKGROUND AND FUNDAMENTALS}

\subsubsection{Bimolecular recombination and Langevin theory}

A bimolecular [or, second-order] recombination process can be understood as an annihilation of two oppositely charged particles [e.g., electrons and holes], hence the [net-] recombination rate $R$ [typically in the units of $\mathrm{cm}^{-3} \mathrm{~s}^{-1}$ ] depends on both electron $[n]$ and hole $[p]$ bulk densities: $R=k_{\text {bulk }} n p$. Here, $k_{\text {bulk }}$ denotes the bimolecular recombination coefficient [in the units of $\mathrm{cm}^{3} \mathrm{~s}^{-1}$ ] and is associated with the electron [hole] carrier lifetime $\tau_{\mathrm{e}}=\left[k_{\mathrm{bulk}} p\right]^{-1}\left[\tau_{\mathrm{p}}=\left[k_{\mathrm{bulk}} n\right]^{-1}\right]$. As a direct consequence, only carriers with $\tau_{\mathrm{e}}>\tau_{\mathrm{tr}}\left[\tau_{\mathrm{p}}>\tau_{\mathrm{tr}}\right]$ can be extracted at the electrodes. Alternatively, one can define the recombination probability based upon the balance between the carrier Coulomb capture radius and their mean free path. Here, the so-called Coulomb capture radius [also referred to as Onsager radius], $r_{\mathrm{c}}=q^{2}\left[4 \pi \varepsilon \varepsilon_{0} k_{\mathrm{B}} T\right]^{-1}$, defines the minimum distance, at which electron and hole do not interact with one another. From an energetic point of view, the Coulomb capture radius can be pictured as the distance at which the temperature-induced kinetic energy of a charge $\left[E_{\text {therm }}=k_{\mathrm{B}} T\right]$ equals the Coulomb potential energy, $E_{\mathrm{C}}=q^{2}\left[4 \pi \varepsilon \varepsilon_{0} r\right]^{-1}$ - hence, electrons and holes are condemned to recombine as soon as they encounter. Assuming a dielectric constant of $\varepsilon=3$ and $k_{\mathrm{B}} T \approx 25 \mathrm{meV}$ [i.e., thermal energy at room temperature], the Coulomb capture radius is $\sim 20 \mathrm{~nm}$.

The bimolecular recombination probability is typically described in accordance with Langevin theory from 1903. [71, 72] Langevin described the recombination probability of two oppositely charged ions in a simple gas as a function of their distance. Here, the recombination coefficient depends on the recombination cross section of mobile carriers $\left[\sigma_{\mathrm{r}}=4 \pi r^{2}\right]$ and the carrier velocity $\left[\nu=\mu E_{\mathrm{C}}(q r)^{-1}\right]$. The Langevin recombination coefficient $\left[k_{\mathrm{L}}\right]$ can be expressed as $k_{\mathrm{L}}=e\left(\mu_{\mathrm{e}}+\mu_{\mathrm{h}}\right)\left[\varepsilon \varepsilon_{0}\right]^{-1}$. It is worth noting that $k_{\mathrm{L}}$ defines an upper limit, and while the Langevin theory successfully describes bimolecular recombination processes observed in single-layer [often neat] organic solar cells, donor: acceptor blended BHJ systems [where charge encounter occurs at donor: acceptor interfaces only] show a suppressed recombination coefficient $k_{\text {bulk }} \ll k_{\mathrm{L}}$. This suppression of the Langevin recombination coefficient is accounted for by introduction of a so-called Langevin reduction factor $\zeta_{\mathrm{L}}=k_{\text {bulk }}\left[k_{\mathrm{L}}\right]^{-1}$, which is found to take values between $10^{-3}$ and 1. 73-75] Koster et al. postulated a mobility-limited charge recombination in BHJ solar cells, where the faster carriers [i.e., those arriving first at a BHJ interface] must 
wait for the slower carrier, before recombination can take place. [76] The corresponding encounter rate coefficient is then given by $k_{\text {slower }}=e \mu_{\text {slower }}\left[\varepsilon \varepsilon_{0}\right]^{-1}$. However, it is worth noting that the applicability of encounter limited recombination depends on the donor: acceptor interconnected network and phase separations. As such, while Langevin recombination is dominant at small phase separations [typically less than 5 $\mathrm{nm}$ ], recombination processes in BHJ systems with large phase separation were found to depend on the slower carrier mobility. In the limit of moderate phase separation [i.e., between $10 \mathrm{~nm}$ and $35 \mathrm{~nm}$, respectively], the recombination rate coefficient was assumed to be reflected by the geometrical mean of the mobilities [i.e., $k \propto \sqrt{\mu_{\mathrm{n}} \mu_{\mathrm{p}}}$ ]. [77] 


\section{THEORETICAL BACKGROUND AND FUNDAMENTALS}

\subsubsection{Monomolecular recombination and trap states}

Monomolecular [or, first-order] recombination is a non-geminate recombination process taking place with one reactant only. Here, the recombination rate $R=d n / d t$ depends linearly on one charge carrier density [e.g., the electron density, $n$ ], and can be expressed as $R_{\text {mono }}=d n / d t=k_{\text {mono }} n$, where $k_{\text {mono }}$ denotes the monomolecular recombination coefficient. If trap states in the bulk of a solar cell are present, either being hole traps [referred to as donor-like trap states, which are neutral (positively charged) when occupied (empty)], or electron traps [referred to as acceptor-like trap states, which are negatively charged (neutral) when occupied (empty)], first-order recombination may take place via those states. The corresponding trap-mediated recombination is commonly described in accordance with Shockley-Read-Hall [SRH] statistics[78], where the associated bimolecular recombination rate coefficient is given by:

$$
\beta_{\mathrm{SRH}}=\frac{C_{\mathrm{n}} C_{\mathrm{p}} N_{\mathrm{t}}}{C_{\mathrm{n}}\left(n+n_{1}\right)+C_{\mathrm{p}}\left(p+p_{1}\right)}
$$

where $n_{1}=N_{\mathrm{C}} \exp \left[\left(E_{\mathrm{t}}-E_{\mathrm{C}}\right)\left[k_{\mathrm{B}} T\right]^{-1}\right]$ and $p_{1}=N_{\mathrm{V}} \exp \left[\left(E_{\mathrm{V}}-E_{\mathrm{t}}\right)\left[k_{\mathrm{B}} T\right]^{-1}\right]$ define the electron and hole concentration in conduction and valance band in the case that the Fermi-level and corresponding trap energy level coincide. Here, $E_{\mathrm{t}}$ defines the [monoenergetic] trap state energy level and $N_{\mathrm{t}}$ is the density of [available] trap states [typically in the unit of $\mathrm{cm}^{-3}$ ]. The coefficients $C_{\mathrm{n}}$ and $C_{\mathrm{p}}$ correspond to the electron and hole capture [or trapping] coefficients and are defined as $C_{\mathrm{n}}=e \mu_{\mathrm{n}}\left[\varepsilon \varepsilon_{0}\right]^{-1}$ and $C_{\mathrm{p}}=e \mu_{\mathrm{p}}\left[\varepsilon \varepsilon_{0}\right]^{-1}$. In the case of shallow trap states, $E_{\mathrm{F}}<E_{\mathrm{t}}$ and thus, $n_{1} \gg n, p$ leading to a recombination rate coefficient $\beta_{\mathrm{SRH}}$ that simplifies to $\beta_{\mathrm{SRH}} \approx C_{\mathrm{p}} N_{\mathrm{t}}\left[n_{1}\right]^{-1}$. Hence, the trap-assisted recombination rate, $R_{\mathrm{SRH}}=\beta_{\mathrm{SRH}} n p$, becomes effectively bimolecular. Deep trap states, on the other hand, refer to the case when $E_{\mathrm{F}}>E_{\mathrm{t}}$, and thus, $n_{1} \ll n, p$. Assuming $n \approx p$, the SRH recombination rate coefficient can then be expressed as $\beta_{\mathrm{SRH}}=C_{\mathrm{p}} C_{\mathrm{n}} N_{\mathrm{t}}\left[n\left(C_{\mathrm{n}}+C_{\mathrm{p}}\right)\right]^{-1}$ and the SRH recombination becomes effectively monomolecular. Based upon the expression for $C_{\mathrm{n}}\left[C_{\mathrm{p}}\right]$ and $n_{1}\left[p_{1}\right]$, the so-called trap release time, $\tau_{\text {rel }}$, [defined as the time after which a trapped charge is released by thermal activation] can be estimated as follows: [79]

$$
\tau_{\text {rel }}=\frac{1}{C_{\mathrm{n}} N_{\mathrm{C}}} \exp \left[\frac{\Delta_{\mathrm{t}}}{k_{\mathrm{B}} T}\right],
$$


where $\Delta_{\mathrm{t}}$ is the trap depth [defined as the energetic difference between trap state and transport level edge]. Assuming typical values of $\mu=10^{-4} \mathrm{~cm}^{2} \mathrm{~V}^{-1} \mathrm{~s}^{-1}, \varepsilon=3$, $N_{\mathrm{C}}=10^{20} \mathrm{~cm}^{-3}$, and $k_{\mathrm{B}} T \approx 0.025 \mathrm{eV}$ [i.e., thermal energy at room temperature], Eq. $\mathbf{1 . 2 3}$ then becomes $\tau_{\text {rel }} \approx 1.7 \times 10^{-10} \exp \left[\frac{\Delta_{\mathrm{t}}}{0.025}\right] \mathrm{s}$ [with $\Delta_{\mathrm{t}}$ given in $\left.(\mathrm{eV})\right]$. Tab. $\mathbf{1 . 1}$ lists rounded trap release times for different trap depths, as expected for above values and calculated along [79].

\begin{tabular}{c||c}
\hline Trap depth $\Delta_{\mathrm{t}}$ in $[\mathrm{eV}]$ & Trap release time $\tau_{\text {rel }}$ \\
\hline 0.05 & $10^{-9} \mathrm{~s}$ \\
0.23 & $10^{-6} \mathrm{~s}$ \\
0.41 & $10^{-3} \mathrm{~s}$ \\
0.58 & $1 \mathrm{~s}$ \\
0.69 & $1 \mathrm{~min}$ \\
0.79 & $1 \mathrm{~h}$ \\
0.88 & $1 \mathrm{~d}$ \\
1.03 & $1 \mathrm{y}$ \\
1.59 & $1 \mathrm{ae}$ \\
\hline
\end{tabular}

Table 1.1: Trap release times $\tau_{\text {rel }}$ estimated from Eq. 1.23 and listed for different trap depths $\Delta_{\mathrm{t}}$ assuming of $\mu=10^{-4} \mathrm{~cm}^{2} \mathrm{~V}^{-1} \mathrm{~S}^{-1}, \varepsilon=3, N_{\mathrm{C}}=10^{20} \mathrm{~cm}^{-3}$, and $k_{\mathrm{B}} T \approx 0.025$ $\mathrm{eV}$ [room temperature]. Trap release times vary between seconds [s], minutes [min], hours [h], days [d], years [y], and the age of the universe [ae, approximately 13.77 billion years].

It becomes clear that when the trap depth starts to exceed $\sim 0.7 \mathrm{eV}$, the trap release time becomes extraordinary long [increasing from seconds (s), minutes (min), hours (h), days (d), years (y) to the age of the universe (ae; approximately 13.77 billion years)]. Hence, the contribution of trap states to charge transport processes needs to be considered when probing the response [e.g., the photocurrent] of an optoelectronic device - especially when probed with signal-alternating measurement techniques. 


\section{THEORETICAL BACKGROUND AND FUNDAMENTALS}

\subsection{Motivation and scope}

Both generation and recombination of charge carriers play a central role in the working principle of thin-film solar cells, and set, along with absorption, charge transport and collection, the overall efficiency of any optoelectronic device. While the low permittivity of organic semiconductors causes free charge generation in these excitonic system to be incomplete, the disordered nature of low-mobility, organic materials leads to a hopping-like charge transport, and carrier loss channels predominantly set by recombination and the diffusion-governed build-up of space-charge. These subtle, but important dissimilarities in electro-optical properties between low-mobility, disordered organic, and high-mobility, crystalline inorganic semiconductors limit measurement techniques, which were previously developed to probe inorganic semiconductors and solar cells, to directly apply to their organic counterparts. However, to further enhance the performance of organic photovoltaic devices through optimization of fabrication processes, it is essential to examine the generation and recombination processes precisely, and to correctly evaluate the findings. Motivated by this challenge, the aim of the work presented in this thesis is to provide tools for probing charge generation and recombination processes in solar cells composed of organic semiconductors accurately, and to convey pathways for a better understanding of the physical processes of charge generation and recombination.

In Chapter 2, electrical and optical noise sources limiting the dynamic range of external quantum efficiency [EQE] measurements will be identified and discussed from an apparatus and device perspective. It will be demonstrated, how the sensitivity of EQE measurements can be enhanced when minimizing the influence of these parasitic noise sources, and dynamic ranges as large as $100 \mathrm{~dB}$ can be achieved. Ultra-sensitive EQE measurements conducted on inorganic, organic and perovskite photovoltaic devices will be used to reveal the contribution of various intra-and intermolecular species to photocurrent generation. In Chapter 3, a newly developed approach for accurate determination of charge generation quantum yields in thin-film organic solar cells based upon temperature dependent, ultra-sensitive EQE measurements, will be the subject matter. Apart from applying the new methodology to organic, state-of-the-art nonfullerene acceptor based thin-film solar cells, the subtle link between charge generation and carrier bulk recombination will be discussed, and applied to organic BHJs resulting 
in high-efficient, thick-junction organic solar cells. In Chapter 4, a new measurement technique, that is, low-intensity integral-time-of-flight [LIITOF], will be described, and demonstrated on thin-film organic and perovskite solar cells. This easy-to-use technique is based upon the integral-time-of-flight technique, but extended to the low-intensity regime, and combined with device capacitance measurements. The field dependence of charge generation will be examined, and results will be correlated to those obtained via time-delayed collection field [TDCF] technique.

While chapter $2-4$ will be mainly linked to photocurrent generation, the aspect of charge carrier recombination will be of subject matter in chapters 5 and 6 . As such, Chapter 5 will focus on the light intensity dependence of thin-film optoelectronic devices, and the influence of different photocurrent loss mechanisms will be reviewed. Herein, the photocurrent loss-inducing character of trap-assisted recombination, the build-up of space-charge due to imbalanced mobilities, the series resistance limitation, and bimolecular recombination will be clarified. Furthermore, it will be highlighted how intensity dependent photocurrent [IPC] measurements, when performed sensitively over a broad range of light intensity, can be used to identify predominant recombination and photocurrent loss mechanisms in thin-film optoelectronic devices. Equipped with the theoretical framework of photocurrent intensity dependence, and the sensitive IPC measurement technique, a comprehensive study on recombination mechanisms in fullerene and non-fullerene acceptor based organic solar cells will be described in Chapter 6. Universally present trap states in the donor: acceptor bulk will be revealed, and their energetics and contribution to photocurrent losses under operational conditions will be quantified. By combining sensitive IPC measurements with ultra-sensitive EQE measurements, the charge-generating and photocurrent-loss inducing character of trap states will be exposed, and the subtle link between the fundamental process of charge generation and recombination will be revealed.

Appendix A will be equipped with information to device fabrication, names and chemical structures of materials, photovoltaic parameter statistics, and drift-diffusion [DD] model parameters; Appendix B will contain additional experimental and DD simulation data and figures. All DD simulations were conducted by Dr. O. J. Sandberg. Unless otherwise noted, detailed information to the DD model are provided in [80] and [81]. 


\section{Chapter 2}

\section{Sensitivity of sub-gap external quantum efficiency measurements}

The measurement of the external quantum efficiency [EQE] for photocurrent generation at photon energies below the bandgap of semiconductors has always been an important tool for understanding phenomena such as charge photogeneration via tail and trap states. The shape of the sub-gap EQE can also reveal the subtle but important physics of inter- and intramolecular states that lay at the heart of charge photogeneration in molecular systems such as organic semiconductors. In this chapter, the influence of optical and electrical noise on the sensitivity of EQE measurements under electrical and optical bias conditions are examined. It is demonstrated how the dynamic range of the EQE apparatus can be enhanced to an unprecedented $>100 \mathrm{~dB}$. Several apparatusand device-related factors limiting the sensitivity including the electrical noise floor of the measurement system and probe light source stray light are discussed and elucidated. By understanding and minimizing the influence of these factors, EQE signals derived from weak sub-gap absorption features in organic, inorganic and perovskite solar cell systems at photon energies well below their bandgaps can be detected.

This chapter is written based upon a collaborative work published by the author in the journal ACS Photonics in 2020 [82]. 


\section{SENSITIVITY OF SUB-GAP EXTERNAL QUANTUM EFFICIENCY MEASUREMENTS}

\subsection{Sub-gap absorption in disordered semiconductors}

Sensitive measurements of photocurrent in solar cells and photodetectors is becoming increasingly important for achieving a better understanding of the mechanisms of light absorption and charge generation. This is particularly the case for semiconductors [such as organics] which have a high degree of disorder. Accurate measurement of photocurrents, and in particular the external quantum efficiency [EQE], below the semiconductor optical bandgap is challenging because the absorption coefficients are orders of magnitude smaller than those measured at energies above the gap. In most crystalline inorganic and perovskite semiconductor devices [such as solar cells and photodetectors] the sub-gap EQE reveals information about the sub-band states such as traps [83, 84] and energetic disorder [85, 87]. In the equivalent organic semiconductor devices, the sub-gap EQE is often used to investigate so-called charge transfer [CT] states. [50, 60, 61] The contribution of these sub-gap states to the photocurrent also plays an important role in the determination of the radiative limit of the open-circuit voltage. 88

In several seminal works, the sub-gap photocurrent has been determined using Fourier transform photocurrent spectroscopy as a fast and sensitive method. 89 91] This technique can reveal the contribution of photo-active species to the photocurrent with absorption coefficients orders of magnitude lower than direct band-to-band transitions. These and related EQE measurements have also proven to be sensitive enough to quantify the contribution of sub-gap states such as the CT states in organic semiconductors and thus, have made a significant impact on the understanding of the mechanism of charge generation in organic optoelectronic devices and particularly the physics of CT states 61, as previously discussed in Chapter 1, Section 1.5. For example, by measuring the EQE sensitively in combination with photo-thermal deflection spectroscopy [PDS] Vandewal et al. have shown relaxed CT states [directly excited by low energy photons] can contribute to the photocurrent as efficiently as those CT states populated via singlet excitons with energies in excess of the CT state energy.[61] Sensitive EQE measurements are now often used in the literature for determination of CT state energies as well as their reorganization energies. [66, 92] Absorption coefficients and crosssections of CT states have also been inferred from sensitive EQE measurements. [54] All of these parameters are crucial for understanding the open-circuit voltage of organic 
solar cells and to determine non-radiative voltage losses versus CT state energy in conjunction with electroluminescence measurements via the detailed balance principle. [93]

The importance of measuring EQE spectra sensitively is thus increasingly appreciated, and motivated the work described in this chapter. In this regard, the aim was to push the boundaries of EQE sensitivity by understanding what fundamentally limits its measurement and thus how to improve it. To achieve this, a commercial spectrophotometer with a double monochromator architecture was used as an ultra-stable, wide spectral range light source, and inorganic, organic, and perovskite solar cells as model test systems were examined to probe noise and sensitivity. The influential and EQE sensitivity-limiting characters of electrical and optical noise sources were demonstrated, and simple means to reduce them and thus increase the minimum detectable EQE were proposed. This is especially important for measuring EQE sensitively under white light or electrical bias, where the device electrical shot noise or light bias optical shot noise are limiting factors. Finally, it is shown that with an apparatus limit of 100 $\mathrm{dB}$ [equivalent to a minimum detectable EQE on the order of $10^{-10} \mathrm{~Hz}^{-1 / 2}$ ], EQEs can be measured down to the thermal noise limit at short-circuit, the electrical shot noise limit under electrical bias, and the photon shot noise for white-light biased devices. 


\section{SENSITIVITY OF SUB-GAP EXTERNAL QUANTUM EFFICIENCY MEASUREMENTS}

\subsection{Basic considerations for sensitivity and dynamic range}

The responsivity, $R$, is defined as the first derivative of the device photocurrent $I$ with respect to incident light power $L$ :

$$
R=\frac{d I}{d L}=\frac{q \lambda}{h c} \times \frac{\phi_{\mathrm{e}}}{\phi_{\mathrm{ph}}}
$$

where $\lambda$ is the excitation wavelength, $q$ the elementary charge, $h$ the Planck constant and $c$ the speed of light in vacuum. Here, $\phi_{\mathrm{e}}$ denotes the flux of extracted photogenerated electrons, whereas $\phi_{\mathrm{ph}}$ denotes the flux of incident photons of energy $\frac{h c}{\lambda}$. The ratio of $\phi_{\mathrm{e}}$ and $\phi_{\mathrm{ph}}$ is then defined as the external quantum efficiency [EQE]:

$$
\mathrm{EQE}=\frac{\phi_{\mathrm{e}}}{\phi_{\mathrm{ph}}}=\frac{h c}{q \lambda} \times R(\lambda)
$$

An important quantity characterizing an EQE measurement system and its ability to detect weak EQE signals sensitively over several orders of magnitude is the dynamic range $[\mathrm{DR}]$ defined as the ratio of the highest $\left[\mathrm{EQE}_{\max }\right]$ and lowest $\left[\mathrm{EQE}_{\min }\right]$ signal that is detectable above the noise floor. Assuming $\mathrm{EQE}_{\max }$ to be 1 [i.e., in absence of any gain], the $\mathrm{DR}$ is then ultimately depending on $\mathrm{EQE}_{\text {min }}$ :

$$
\mathrm{DR}=10 \times \log \left[\frac{1}{\mathrm{EQE}_{\min }}\right]=10 \times \log \left[\frac{q \lambda}{h c} \times \frac{L(\lambda)}{I_{\text {noise }}}\right],
$$

where $I_{\text {noise }}$ [given by $I_{\mathrm{NSD}} \times \sqrt{\Delta f}$ with $I_{\mathrm{NSD}}$ being the noise current spectral density (NSD) and $\Delta f$ denoting the electrical bandwidth] is the device average noise current. While most conventional solar cells are designed to achieve the highest EQE in the visible [Vis] wavelength regime, $\mathrm{EQE}_{\text {min }}$ will be typically located at longer wavelengths [i.e., at photon energies below the bandgap] in the near infrared [NIR]. Subsequently, achieving a high DR [low $\mathrm{EQE}_{\min }$ ] requires in the first instance a light source with particularly high power in the NIR and secondly, a low noise current $I_{\text {noise }}$. It is worth noting that $I_{\text {noise }}$ may be frequency dependent if flicker noise is present and at large enough frequencies it is determined by the thermal noise [dependent on the device shunt resistance] and/or the shot noise [see Box 2.1. 93 96] 
From Eq. 2.2 it follows that the noise equivalent EQE is given by:

$$
\mathrm{EQE}_{\mathrm{NE}}=\frac{h c}{q \lambda} \times \frac{I_{\mathrm{NSD}} \sqrt{\Delta f}}{L} .
$$

It is worth noting that, as a consequence of Eq. 2.4, every experimentally measured EQE signal $\left[\mathrm{EQE}_{\exp }\right]$ can be assigned to either a real [i.e., $\mathrm{EQE}_{\exp }>\mathrm{EQE}_{\mathrm{NE}}$ ] or noise [i.e., $\left.\mathrm{EQE}_{\exp } \leq \mathrm{EQE}_{\mathrm{NE}}\right]$ signal.

\section{Background information to noise current}

\section{Box 2.1}

The noise current $i_{\text {noise }}$ of any optoelectronic device can be calculated from the sum of shot, thermal and flicker noise:

$$
\left\langle i_{\text {noise }}^{2}\right\rangle=\left\langle i_{\text {shot }}\right\rangle^{2}+\left\langle i_{\text {thermal }}\right\rangle^{2}+\left\langle i_{\text {flicker }}\right\rangle^{2}
$$

While flicker noise is the predominant noise term at low frequencies, at large enough frequencies, $i_{\text {flicker }}$ is negligibly small and Eq. 2.5 can be written as:

$$
\left\langle i_{\text {noise }}^{2}\right\rangle=[\underbrace{2 q \Delta f i_{\mathrm{d}}}_{\text {shot noise }}+\underbrace{\frac{4 k_{\mathrm{B}} T \Delta f}{R_{\text {shunt }}}}_{\text {thermal noise }}], 97
$$

where $i_{\mathrm{d}}$ denotes the average dark current, $k_{\mathrm{B}}$ is the Boltzmann constant, $T$ the temperature, and $R_{\text {shunt }}$ defines the device shunt resistance. Here, shot noise is related to the random arrival of charge carriers, while thermal noise is related to fluctuations of charge carrier densities in the circuit. It follows that the lower the noise current, the higher dynamic range, $\mathrm{DR} \propto \log \left[\frac{1}{I_{\text {noise }}}\right]$. 


\section{SENSITIVITY OF SUB-GAP EXTERNAL QUANTUM EFFICIENCY MEASUREMENTS}

\subsection{Home-built, ultra-sensitive EQE apparatus}

Fig. 2.1 shows a schematic of the system for the high sensitivity EQE measurements. In the apparatus, a commercial, high-performance spectrophotometer Lambda950 from PerkinElmer, conventionally used for reflection, transmission and absorption measurements, acts as the [probe] light source. A combined deuterium and tungsten halogen lamp [LS1 in Fig. 2.1] provide an extended spectral wavelength regime from 175 $\mathrm{nm}$ up to $3300 \mathrm{~nm}$. Several different optical components, such as filters, mirrors and lenses, together with two double holographic grating monochromators providing a spectral output [slit] width of $5 \mathrm{~nm}$ in VIS and $20 \mathrm{~nm}$ in NIR, and are used to provide an ultra-low stray light source with high temporal stability and flexibility to control spot size and polarization.

\section{Background information to lock-in method}

\section{Box 2.2}

The lock-in method describes a phase-sensitive detection and measurement technique of very small alternate current $[\mathrm{AC}]$ signals. Herein, the input signal of a device under test [DUT] is modulated at a certain reference frequency, $\omega_{\text {ref }}=2 \pi f_{\text {ref. }}$ It is worth noting that the input signal can contain a variety of other frequency-components [at frequency $f_{\mathrm{i}}$ and amplitude $A_{\mathrm{i}}$ ]. Subsequently, the input signal can be expressed as:

$$
A_{\mathrm{ref}} \sin \left(\omega_{\mathrm{ref}} t\right)+\sum_{\mathrm{i}} A_{\mathrm{i}} \sin \left(\omega_{\mathrm{i}} t\right)
$$

A so-called lock-in amplifier detects the DUT input signal and multiplies a sine wave [with the reference frequency $\omega_{\text {ref }} ; \sin \left(\omega_{\text {ref }} t\right)$ ] to the input signal. For all input signals with $\omega_{i} \neq \omega_{\text {ref }}$, the averaged output signal is zero, leaving only

$$
A_{\mathrm{ref}} \sin \left(\omega_{\mathrm{ref}} t\right) \times \sin \left(\omega_{\mathrm{ref}} t\right)=\frac{A_{\mathrm{ref}}}{2} \times\left[1-\cos \left(2 \omega_{\mathrm{ref}} t\right)\right]
$$

as the remaining output signal part. While the $2 \omega_{\text {ref-component }}$ of the output signal in Eq. 2.8 [right-hand side] is filtered out by lock-in amplifier internal low [high] pass filters, a constant direct current [DC] signal remains. 
The output light from the spectrometer source is physically chopped by a multi-blade chopper wheel [OC in Fig. 2.1] at frequency $f=\frac{\omega}{2 \pi}$ [Thorlabs MC2000B] and different long pass filters [LPF, Edmunds Optics OD4] were used to filter out remaining, parasitic stray light. As shown in Fig. 2.1, switch $\mathrm{S} 1$ allows for the detection of the photocurrent signal with a lock-in amplifier [Stanford Research Systems, SR860] passing through a current pre-amplifier [FEMTO, DLPCA-200] with variable gain [up to $10^{9} \mathrm{VA}^{-1}$ at low noise] and integrated low noise voltage source allowing for adjustable bias voltages to be applied $[ \pm 10 \mathrm{~V}]$. For EQE measurements under open-circuit conditions, switch S2 enables the use of a high input impedance [1 T $\Omega$ ] voltage-pre-amplifier [FEMTO, DLPVA-100-F-S] with variable gain [up to $80 \mathrm{~dB}$ ] in series with the device under test [DUT] [see Fig. 2.1]. A DC blocker [Thorlabs, EF500] is used to maintain the open-circuit condition when light bias is used. This component is not blocking at the frequency $f[\gg 1 \mathrm{~Hz}]$ so that the total DC current is zero [i.e., open circuit] but the $\mathrm{AC}$ component can be measured. The $100 \mathrm{k} \Omega$ resistor in parallel with the voltage pre-amplifier is used to regulate the input impedance of this pre-amplifier to reduce the $R C$-time constant of the circuit. The lock-in amplifier, providing integration times [electrical bandwidths] from $1 \mu \mathrm{s}\left[10^{6} \mathrm{~Hz}\right]$ up to $30 \mathrm{ks}\left[\sim 3.33 \times 10^{-5} \mathrm{~Hz}\right]$, detects the preamplified photocurrent of the DUT. Here, the OC is used as an external reference source for the AC modulation frequency [see Box 2.2. For EQE measurements under light bias, an additional [pump] light source [LS2 in Fig. 2.1] with variable output power can be used to illuminate the DUT. For the calibration process, a Newport NIST calibrated silicon 818-UV [for wavelengths between $200 \mathrm{~nm}$ and $1100 \mathrm{~nm}$ ], germanium 818-IR [for wavelengths between $780 \mathrm{~nm}$ and $1800 \mathrm{~nm}$ ] and Thorlabs indium gallium arsenide S148C [for wavelengths between $1200 \mathrm{~nm}$ and $2500 \mathrm{~nm}$ ] photodiode sensor were used. A resolution and video bandwidth of $1 \mathrm{~Hz}$ were utilized for measurements of the NSD using a Keysight Spectrum Analyzer N9010B [SA in Fig. 2.1] operating under AC-coupling.

\footnotetext{
${ }^{1}$ National Institute of Standards and Technology
} 


\section{SENSITIVITY OF SUB-GAP EXTERNAL QUANTUM EFFICIENCY MEASUREMENTS}

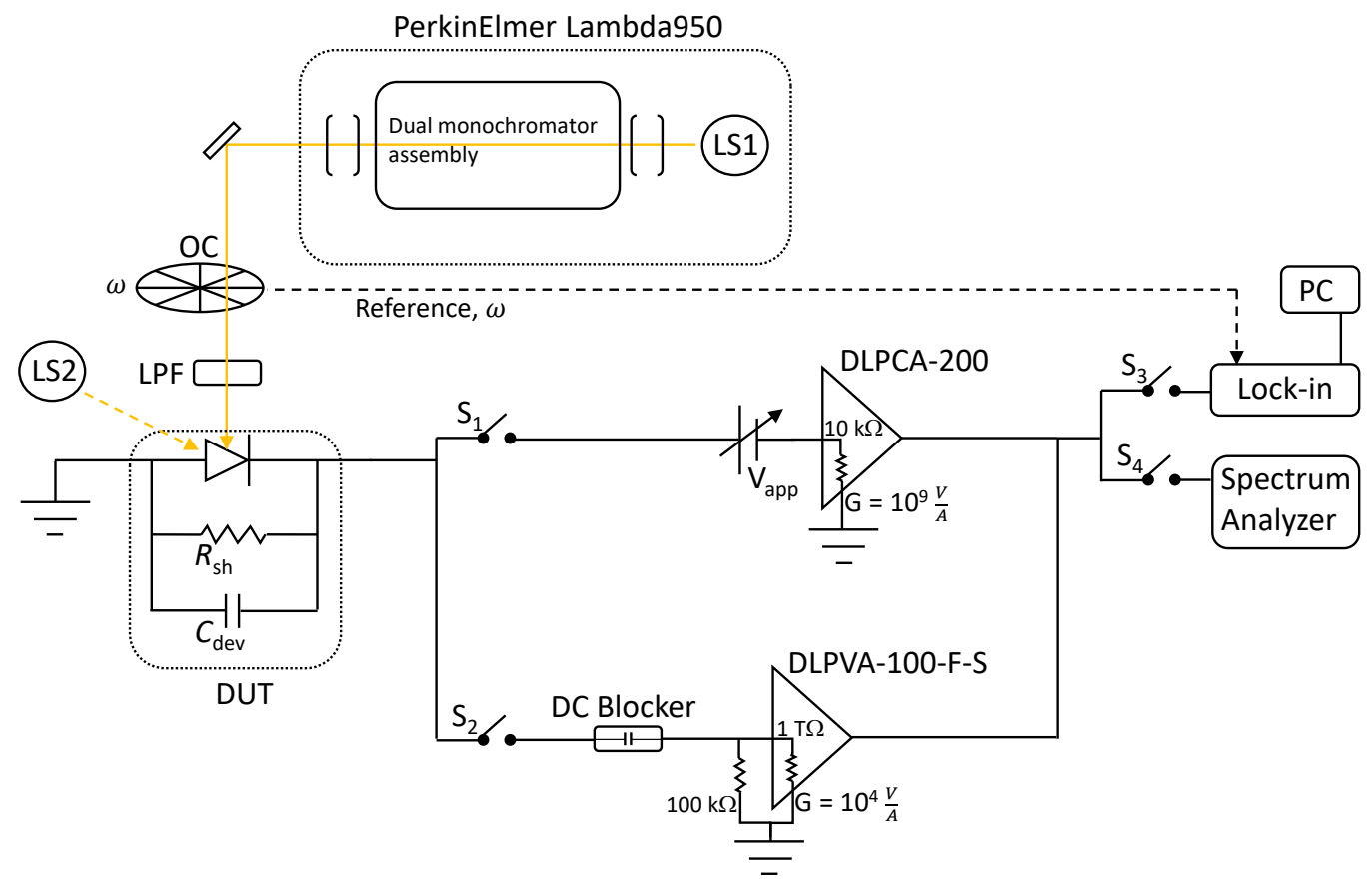

Figure 2.1: Schematic of the experimental setup for sensitive external quantum efficiency [EQE] measurements using a Lambda950 [PerkinElmer] spectrophotometer. An optical chopper $[\mathrm{OC}]$ is used for modulating the monochromatic light source. Prior to focusing the light on the device under test [DUT], different long pass filters [LPF] can be used to filter out parasitic stray light and harmonics of the monochromator. Using switch S1, the current pre-amplifier amplifies the photocurrent signal and allows for biasing the DUT using its internal low noise voltage source. Switch S2 optionally allows for the measurement of EQE under open-circuit conditions using the high input impedance $[1 \mathrm{~T} \Omega]$ voltage pre-amplifier in series with a DC blocker and a $100 \mathrm{k} \Omega$ resistor [corresponds to a gain of $100 \mathrm{~dB}$ ]. A lock-in amplifier is used to read the amplified AC photocurrent signal of the DUT at a specified electrical bandwidth [switch S3]. Switch S4 enables the noise spectral density [NSD] measurements via a spectrum analyser. Optionally, an additional light source [LS2] with variable intensity can be used for measurements of the EQE sensitively under light bias. 


\subsubsection{Dynamic range optimization}

In order to optimize the EQE apparatus and achieve the large DRs needed to detect weak signals at photon energies well below the bandgap, identification of optical and electrical noise sources is of the utmost importance. The electrical noise of the EQE apparatus was examined by measuring the NSD of the [current] pre-amplifier at the highest gain of $10^{9} \mathrm{VA}^{-1}$ as a function of frequency [see Fig. 2.2 a]. The noise level determines the apparatus limit of the EQE measurement when an ideal device [with noise level smaller than the pre-amplifier] is used. In Fig. 2.2 a, the vertical, dashed line marks the frequency of $273 \mathrm{~Hz}$, at which the EQE measurements were performed. Importantly, the frequency of $273 \mathrm{~Hz}$ consciously avoids the flicker noise at very low frequencies $[<10 \mathrm{~Hz}$ ] and the mains hum noise peaks at $50 \mathrm{~Hz}$ and multiples [both flicker and hum reduce at higher frequencies]. The frequency of the measurement must clearly be chosen to be different to the hum noise peaks. Failing to do so will result in a substantial increase to the background noise of the measurement which cannot be improved by lowering the electrical bandwidth as the hum noise peaks will then appear as parasitic 'signal' with constant phase such that they cannot be averaged out by longer integration times. However, one should also be aware that the choice of frequency is not only limited by the technical aspects of the EQE system [e.g., mechanical limitations of the chopper blade or noise peaks of the pre-amplifier], but also by the underlying physical processes of the DUT, such as charge transport and $R C$-time of the device/circuit as well as trapping and de-trapping lifetimes of charge carriers within the active layer. Therefore, higher frequencies greater than the cutoff frequency of the device/circuit combination must additionally be avoided. Failing to do so will result in an underestimation of the EQE. As shown in Fig. 2.2 a, a pre-amplifier NSD of $1.62 \times 10^{-15} \mathrm{AHz}^{-1 / 2}$ [at gain $10^{9} \mathrm{VA}^{-1}$ ] at a frequency of 273 $\mathrm{Hz}$ [see Fig. 2.2 a, vertical dashed line] was measured with the spectrum analyzer under dark conditions using an electrical bandwidth of $1 \mathrm{~Hz}$. Fig. 2.2 b [left axis; black line] shows the output light power $L$ of the Lambda950 measured with calibrated photodiodes. The EQE $\mathrm{NE}_{\mathrm{NE}}$ was calculated from Eq. 2.4 and is shown as the red curve in Fig. 2.2b [right axis]. This represents the fundamental limit of the apparatus at an electrical bandwidth of $1 \mathrm{~Hz}$. As shown in Fig. 2.2 b, a minimum EQE $\mathrm{NE}_{\mathrm{NE}}$ of approximately $1.14 \times 10^{-10} \mathrm{~Hz}^{-1 / 2}$ was achieved at $1200 \mathrm{~nm}$, while an EQE of 2.15 


\section{SENSITIVITY OF SUB-GAP EXTERNAL QUANTUM EFFICIENCY MEASUREMENTS}

$\times 10^{-9}$ was obtained at $2500 \mathrm{~nm}$. It is worth noting that the noise equivalent EQE ultimately depends on the electrical bandwidth [see Eq. 2.4] and subsequently, one has to decrease the electrical bandwidth in order to reduce the total noise, measure a lower EQE and thus achieve a higher DR. This, of course, slows down the speed of the measurement.
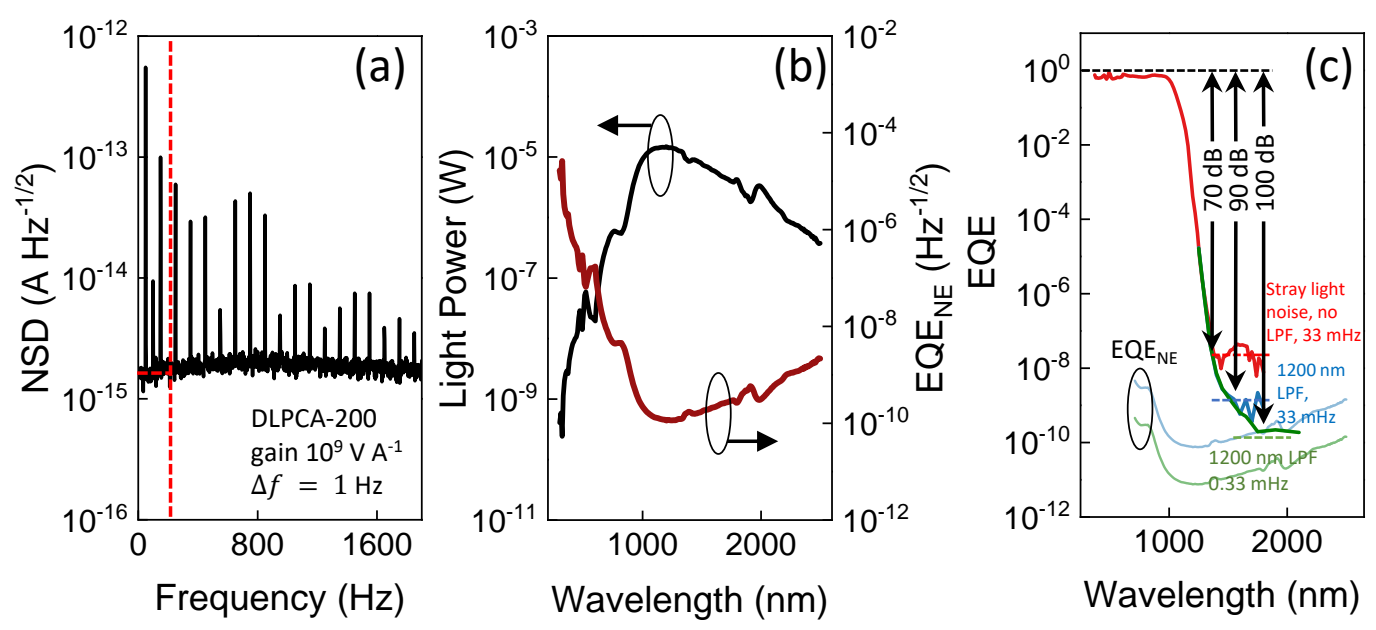

Figure 2.2: (a) Noise spectral density $[\mathrm{NSD}]$ of the current pre-amplifier plotted as a function of frequency. The vertical dashed line marks the chopping frequency of $273 \mathrm{~Hz}$, at which the sensitive external quantum efficiency $[\mathrm{EQE}]$ measurements were performed. Flicker noise is dominant at very low frequencies $[<10 \mathrm{~Hz}]$ and hum noise peaks at harmonics of $50 \mathrm{~Hz}$ are evident. (b) Output power of the Lambda950 spectrophotometer [left axis; black line] measured with calibrated silicon, germanium and indium gallium arsenide photodiodes, and calculated apparatus noise equivalent $\mathrm{EQE}\left[\mathrm{EQE}_{\mathrm{NE}}\right]$ spectrum of the current pre-amplifier [right axis; red line] representing the minimum detectable EQE spectrum of the apparatus at an electrical bandwidth of $1 \mathrm{~Hz}$. (c) EQE and calculated $\mathrm{EQE}_{\mathrm{NE}}$ spectra of a calibrated silicon photodiode measured at different electrical bandwidths and long-pass filters [LPF]. Vertical dotted lines mark the calculated thermal noise limits.

In this regard, it should be noted that the total EQE measurement time depends on both electrical bandwidth and wavelength step size. For large EQE signals [typically at photon energies above the bandgap] the electrical bandwidth [integration time constant] can be increased [decreased] to $1 \mathrm{~Hz}$ [1 s], while for EQE signals below the bandgap the electrical bandwidth is decreased to much lower values down to $0.33 \mathrm{mHz}$ corresponding to a time constant of $3 \mathrm{ks}$. The measurement time is also defined by the relaxation time of the lock-in amplifier which is typically four times larger than the time constant. Subsequently, a total measurement time for a highly sensitive EQE measurement over a 
broad wavelength regime can easily take up to 24 hours to complete. Having understood and characterized the apparatus-limited $\mathrm{EQE}_{\mathrm{NE}}$, a crystalline silicon photodiode [818UV, Newport] was investigated as a DUT [see Fig. 2.2 c]. The EQE measurements were performed at short-circuit at an electrical bandwidth of $33 \mathrm{mHz}$. The EQE $\mathrm{NE}_{\mathrm{NE}}$ was calculated from the noise spectral density of the silicon photodiode and plotted in the same panel as a dotted curve. When using the Lambda950 as a light source only [i.e., without additional long pass filters], the experimentally obtained $\mathrm{EQE}_{\exp }$ [see solid, red line in Fig. 2.2 c] exhibits an apparent sub-bandgap plateau with an EQE of $10^{-7}$. Using an additional $1200 \mathrm{~nm}$ long pass filter, this plateau disappears and the $\mathrm{EQE}_{\exp }$ agrees with the thermal-noise-limited $\mathrm{EQE}_{\mathrm{NE}}$ at long enough wavelengths [see solid, blue line in Fig. 2.2 c]. This implies that in absence of the long-pass filter the minimum detectable EQE was limited by the optical [parasitic stray light] noise of the monochromator which in this case was on the order of $-70 \mathrm{~dB}$ relative to the main monochromator signal. To further improve the sensitivity, a 100 times lower electrical bandwidth of $0.33 \mathrm{mHz}$ was used resulting in an $\mathrm{EQE}_{\mathrm{NE}}$ at $1800 \mathrm{~nm}$ of approximately $10^{-10}$ [see solid, green line in Fig. 2.2 c] and a DR of $100 \mathrm{~dB}$ was thus achieved. 


\section{SENSITIVITY OF SUB-GAP EXTERNAL QUANTUM EFFICIENCY MEASUREMENTS}

\subsubsection{The influence of optical bias on sensitivity}

In the following section, the sensitive EQE under light bias conditions is discussed. Under these conditions, the overall noise current is limited by the thermal noise of the device and the photon shot noise of the light bias source with the latter being dominant at relevant light intensities up to 1-sun equivalent illumination. Fig. 2.3a shows the NSD of an organic PBDB-T:ITIC solar cell plotted as a function of short-circuit current density, $J_{\mathrm{sc}}$. Details to device fabrication and dark [light] $J$ - $V$ curves are provided in Appendix $\mathbf{A}$ and Fig. B.1 in Appendix B. Different short-circuit current densities were obtained by varying the output power of three different bias light sources - a laser diode, a light-emitting diode [LED], and a Halogen lamp. The dashed line in Fig. 2.3a represents the ideal NSD of the PBDB-T:ITIC solar cell as if it was light-biased with a shot noise-limited light source [i.e., an ideal laser] with the photon noise of $\delta n=\sqrt{\langle n\rangle}$, where $\langle n\rangle$ is the average photon number. It is worth noting that an ideal laser is shot noise-limited and clearly, the laser used here is far from that limit. The $520 \mathrm{~nm}$ laser diode source exhibits the largest noise which is approximately three orders of magnitude higher than the photon shot noise. The Halogen lamp exhibits the lowest noise and about 10 times larger than the photon shot noise of an ideal coherent source. A thermal light source exhibits super-Poissonian photon statisticst hence photon noise larger than the shot noise is expected. Based upon the measured noise currents under illumination the $\mathrm{EQE}_{\mathrm{NE}}$ spectra for the three given different light sources can be calculated.

The results for the calculated $\mathrm{EQE}_{\mathrm{NE}}$ spectra under light bias with different light sources are shown in Fig. 2.3b. The dahsed line represents the expected $\mathrm{EQE}_{\mathrm{NE}}$ for the PBDB-T:ITIC solar cell when a shot-noise-limited light source is used. Amongst the three light sources, the halogen lamp exhibits the lowest noise thus lower EQE $E_{\mathrm{NE}}$. Therefore, for the rest of the experiment this light source was employed. Here the importance of the choice of the bias light source for measuring light-biased sensitive EQEs should be emphasized. A thermal light source such as a halogen lamp is often accessible whilst low noise [i.e., shot-noise-limited] lasers with high enough power are rather expensive. By using an intensity-squeezed light source where the photon statistics are sub-Poissonian ${ }^{\square}$ [hence photon noise below the shot noise], the EQE $\mathrm{NE}_{\mathrm{NE}}$

\footnotetext{
${ }^{1}$ Based on the relation of average photon number $\langle n\rangle$ to variance $\delta n$, photon statistics of light sources are classified as Poissonian [i.e., $\delta n^{2}=\langle n\rangle$ ], sub-Poissonian [i.e., $\delta n^{2}<\langle n\rangle$ ], and superPoissonian [i.e., $\left.\left.\delta n^{2}\right\rangle\langle n\rangle\right]$.
} 
can be further reduced. However, such sources are again expensive and difficult to achieve high enough powers for light biasing. By decreasing the electrical bandwidth similar improvements can be achieved [note, however, that the measurement time then increases].
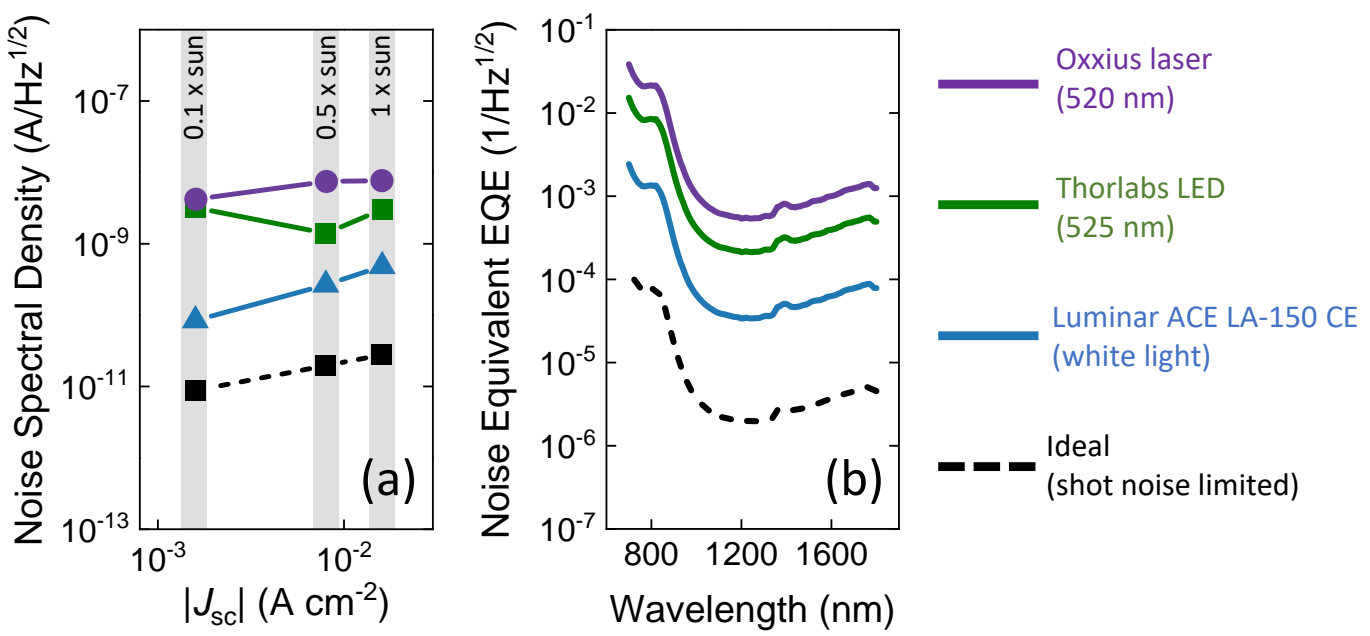

Figure 2.3: (a) Noise spectral density [NSD] at $\Delta f=1 \mathrm{~Hz}$ and $f=273 \mathrm{~Hz}$ of a PBDBT:ITIC solar cell [at short-circuit] plotted as a function of short-circuit current density, and compared for the three different light sources. Increasing the pump light intensity [or, the short-circuit current density, $J_{\mathrm{sc}}$ results in a larger noise current due to the shot noise associated with the DC background current. (b) Calculated noise equivalent external quantum efficiency $\left[\mathrm{EQE}_{\mathrm{NE}}\right]$ spectra under constant 1 sun equivalent pump intensity and compared for three different light sources. The dashed black line represents the ideal noise equivalent EQE which can be only achieved when a shot-noise-limited light source [ideal laser] is used for light-biasing. The $\mathrm{EQE}_{\mathrm{NE}}$ [representing the minimum detectable EQE, normalized to the electrical bandwidth] strongly depends on the [optical] noise of the pump light source.

Fig. 2.4 a shows the EQE $E_{\exp }$ [solid lines] spectra of a PBDB-T:ITIC solar cell measured at zero applied bias voltage [short-circuit] for the different bias powers corresponding to a range from 0 to 1 sun. The red solid lines indicate the EQE $E_{N E}$. The EQE $E_{\exp }$ strongly depends on the shot noise of the solar cell [see Fig. 2.3 a] induced by the DC illumination and limits the sensitivity: while a minimum $\mathrm{EQE}_{\exp }$ at high wavelengths of approximately $10^{-7}$ could be obtained without additional illumination [see black solid line in Fig. 2.4 a], only $10^{-4}$ [see blue solid line in Fig. 2.4 a] could be achieved at a 1 sun equivalent output power. The calculated $\mathrm{EQE}_{\mathrm{NE}}$ again confirms the strongly shot noise limited EQE spectra [see dotted lines in Fig. 2.4 a]. Fig. 2.4 b shows the EQE $E_{\exp }$ 


\section{SENSITIVITY OF SUB-GAP EXTERNAL QUANTUM EFFICIENCY MEASUREMENTS}

of the PBDB-T:ITIC solar cell measured under 1 sun equivalent DC output power of the halogen lamp both under short- and open-circuit conditions, and compared with the EQE spectrum obtained at zero bias without bias illumination. For the measurement of the EQE sensitively under the open-circuit condition, a high impedance voltage pre-amplifier was used [see Fig. 2.1]. The horizontal dashed and dotted lines mark the corresponding noise floors at higher wavelengths. In this case the $\mathrm{EQE}_{\exp }$ obtained under the open-circuit condition is almost 4 orders of magnitude lower than the $\mathrm{EQE}_{\exp }$ measured at short-circuit. Normalization of the $\mathrm{EQE}_{\exp }$ spectra reveals no significant change in the spectral shape of the contribution of intra-and intermolecular states to the EQE between short-and open-circuit conditions.

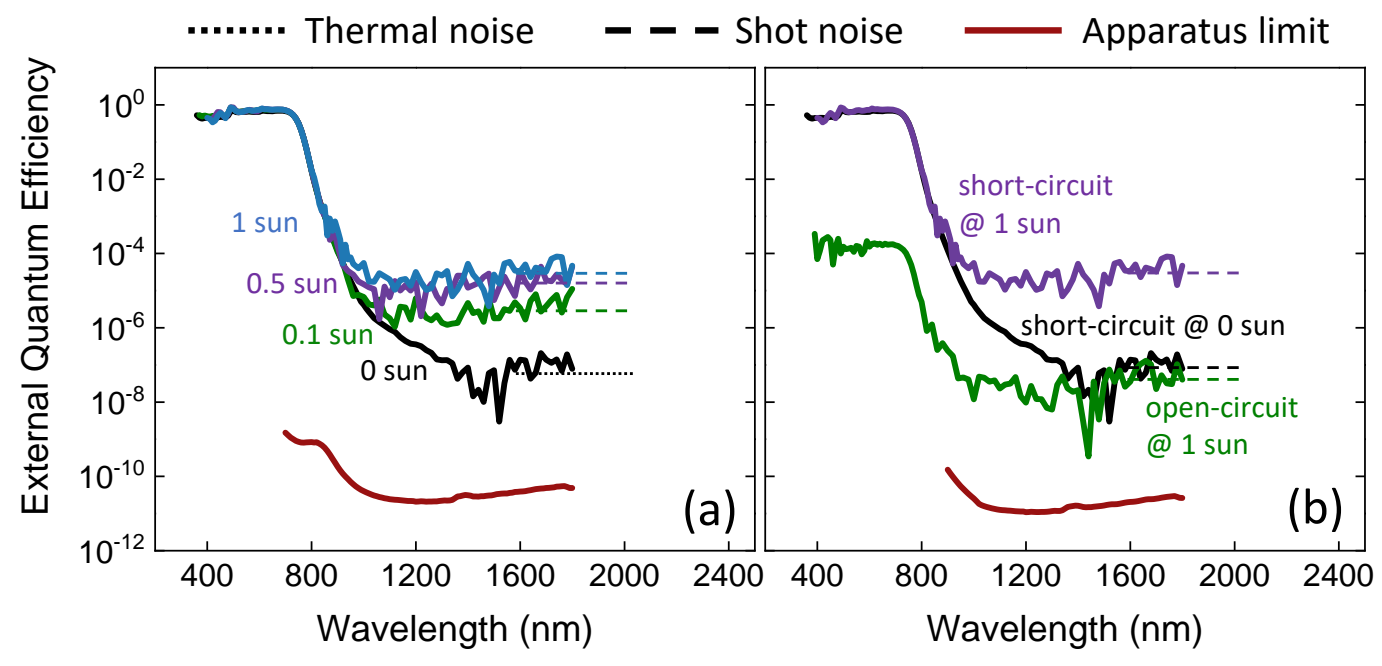

Figure 2.4: (a) External quantum efficiency [EQE] [solid lines] and calculated noise equivalent EQE [EQE $\mathrm{EE}_{\mathrm{NE}}$ ] [red solid line] spectra of a PBDB-T:ITIC solar cell measured at $f=273 \mathrm{~Hz}, \Delta f=33 \mathrm{mHz}$ and $V_{\text {bias }}=0 \mathrm{~V}$, and compared for three different white light Halogen pump illumination intensities. The dotted and dashed lines represent the corresponding calculated noise equivalent EQE spectrum. (b) EQE spectrum of a PBDBT:ITIC solar cell under constant 1 sun equivalent pump intensity measured under shortcircuit and open-circuit, and compared with the EQE spectrum at zero pump intensity [at short-circuit]. The red solid line represent the corresponding calculated noise equivalent EQE spectrum. Dashed and dotted lines mark the corresponding noise floors at higher wavelengths. The chopper frequency was set to $f=273 \mathrm{~Hz}$ and an electrical bandwidth of $\Delta f=33 \mathrm{mHz}$ was used. 


\subsubsection{The impact of electrical bias on dynamic range}

Fig. 2.5a shows the NSD of the PBDB-T:ITIC solar cell measured at a frequency of $273 \mathrm{~Hz}$ and an electrical bandwidth of $1 \mathrm{~Hz}$ plotted as a function of applied bias. All NSD measurements were performed in the dark using a Spectrum Analyzer [SA in Fig. 2.1 and different bias voltages between $-1.5 \mathrm{~V}$ and $0.85 \mathrm{~V}$ were applied using the internal, low noise voltage source of the pre-amplifier. The horizontal solid line in Fig. 2.5a marks the NSD of the pre-amplifier [at the highest gain of $10^{9} \mathrm{VA}^{-1}$ ] of approximately $1.62 \times 10^{-15} \mathrm{AHz}^{-1 / 2}$ and therefore, represents the noise floor of the EQE apparatus. At zero bias [short-circuit] the thermal-noise-limited NSD of the PBDB-T:ITIC solar cell is approximately $1.36 \times 10^{-12} \mathrm{AHz}^{-1 / 2}$.
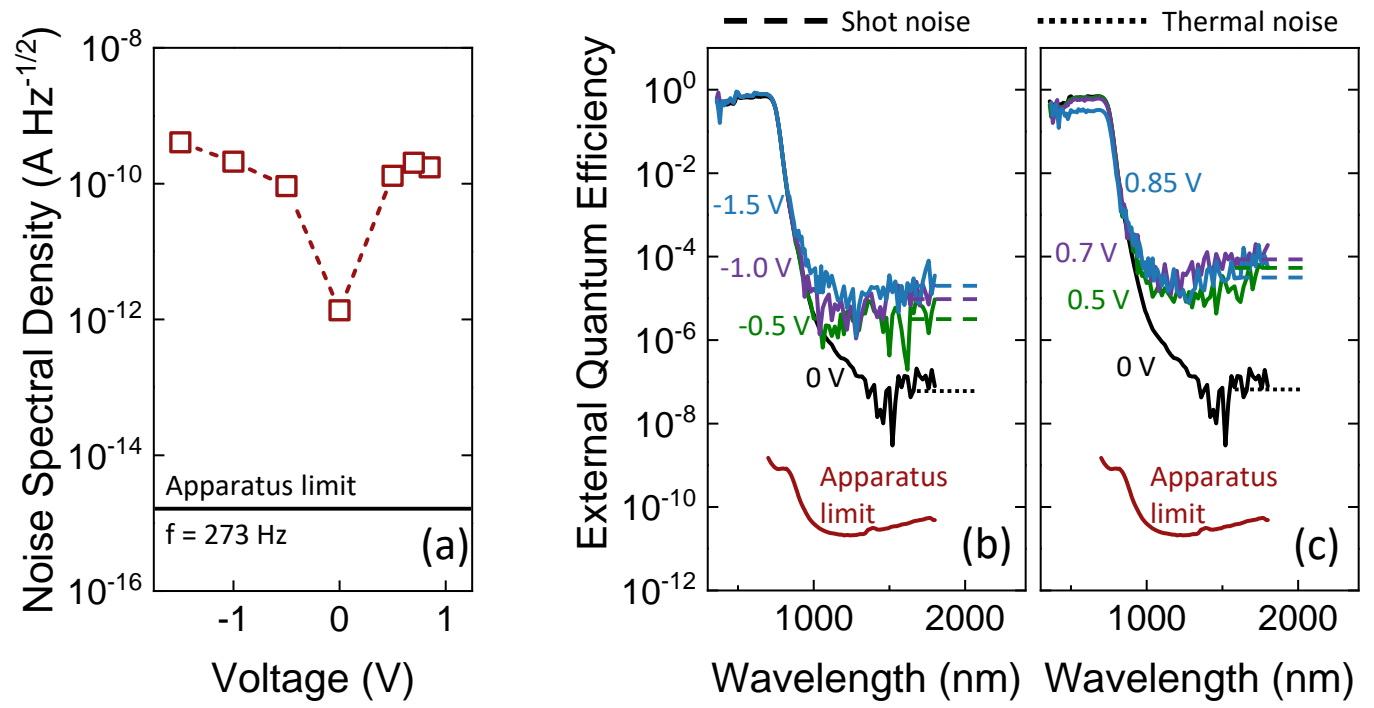

Figure 2.5: (a) Noise spectral density [NSD] of a PBDB-T:ITIC solar cell plotted as a function of the applied bias. Due to the finite shunt resistance of the device, the noise at zero bias [short-circuit] is larger than the apparatus noise floor [horizontal solid line]. Increasing the applied [forward or reverse] bias voltage results in a larger noise current due to the shot noise associated with the background DC current from the applied voltage. (b) External quantum efficiency [EQE] spectra of a PBDB-T:ITIC solar cell measured at four different applied reverse bias voltages [solid lines], and compared with the corresponding calculated noise equivalent EQE spectra $\left[\mathrm{EQE}_{\mathrm{NE}}\right]$ [dotted and dashed lines] for each bias. While at zero bias a minimum EQE of approximately $10^{-7}$ could be achieved, the bias dependent shot noise leads to a minimum EQE of only $10^{-4}$ at an applied voltage of -1.5 V. (c) Repetition of panel b) but under different applied forward biases varying between $0 \mathrm{~V}$ and $0.85 \mathrm{~V}$. 


\section{SENSITIVITY OF SUB-GAP EXTERNAL QUANTUM EFFICIENCY MEASUREMENTS}

Here, the thermal noise due to the finite shunt resistance of the device leads to an increased NSD of approximately three orders of magnitude above the apparatus noise limit. Note that the [thermal] noise current [limit] of the device can be also estimated from the shunt resistance of the device [see Eq. 2.6 in Box 2.1]. As shown in Fig. 2.5a, when increasing the forward or reverse bias, the electrical noise of the PBDBT:ITIC solar cell rapidly increases by almost three orders of magnitude to roughly $10^{-9}$ $\mathrm{AHz}^{-1 / 2}$ at an applied reverse bias of $-1.5 \mathrm{~V}$, respectively $0.85 \mathrm{~V}$. This is due to the shot noise induced by the bias current. Fig. 2.5 b,c show the experimentally obtained $\mathrm{EQE}_{\exp }$ [solid lines] and the noise floors [dotted and dashed lines] corresponding to the $\mathrm{EQE}_{\mathrm{NE}}$ spectra of the PBDB-T:ITIC solar cell measured over a broad range of wavelengths at different applied reverse and forward bias voltages. As shown in Fig. 2.5 b,c, the voltage dependent shot noise of the DUT strongly influences the sensitivity of the EQE measurements: while a minimum $\mathrm{EQE}_{\exp }$ at longer wavelengths of $10^{-7}$ at zero bias could be obtained [see black solid line in Fig. 2.5 b,c), only $10^{-4}$ could be achieved at an applied reverse bias voltage of $-1.5 \mathrm{~V}$ [see blue solid line in Fig. 2.5 b,c], respectively forward bias of $0.85 \mathrm{~V}$. The noise floors shown by the dotted and dashed lines were obtained by blocking the monochromator beam. These values agree with the $\mathrm{EQE}_{\mathrm{NE}}$ spectra calculated from the noise currents of Fig. 2.5 a confirming that the EQE is predominantly limited by the shot noise under electrical bias and by the thermal noise at short circuit condition. 


\subsection{Ultra-sensitive EQE of photovoltaic devices}

These ultra-sensitive EQE measurements obtained through minimizing the electrical and optical noise allowed for the detection of photocurrent at photon energies well below the bandgap of the crystalline silicon photodiode. As shown in Fig. 2.2c, deep mid-gap states are revealed in the EQE spectrum at wavelengths above $1400 \mathrm{~nm}$ [photon energies smaller than $0.89 \mathrm{eV}$ ]. The EQEs of other inorganic optoelectronic devices including crystalline [c-] [part number KXOB22-12X1, IXYS], poly-crystalline [poly-] [JY03264, Shenzhen Yibai Network Technology Co., Ltd.] and hydrogenated amorphous [a-] [TRONY, model number SC8125 S-8] silicon solar cells as well as a germanium [Ge] photodiode [818-IR, Newport] were then examined. These measurements likewise revealed similar sub-gap absorption features at photon energies well below the constituent semiconductor bandgaps [see Fig. 2.6 a]. A mid-gap trap induced tail is revealed in the EQE spectra at extremely low responsivities [-60 $\mathrm{dB}$ to $-80 \mathrm{~dB}$ relative to the above-bandgap EQE] of c-Si and poly-Si solar cells, and the c-Si photodiode. One can also naturally see that in the a-Si:H solar cell the band-edge is much broader than that in c-Si and poly-Si solar cells/photodiodes due to the intrinsic and well-studied energetic disorder. The trap-induced tail of the EQE in a-Si:H is considerably stronger than that of c-Si and poly-Si with three distinct sub-bandgap peaks due to chargedtraps and defect states. [98]

Ultra-sensitive EQE measurements conducted on a large variety of fullerene and nonfullerene acceptor based organic solar cells were subject of a recently published article by Zarrabi et al. in 2020. [49] Here, Zarrabi and co-workers directly observed universally present charge-generating sub-gap trap states via EQE measurements performed sensitively over a broad range of photon energies. The trap states were found to be situated in the middle of the donor: acceptor interfacial bandgap. In this regard, Fig. 2.6b shows the EQE spectrum of an organic PCDTBT:PC ${ }_{70} \mathrm{BM}$ solar cell measured sensitively over a broad range of photon energies.

In 2020, Kaiser et al. developed an approach to determine low finesse cavity-free, ultra-low absorption coefficients in organic semiconductor materials based upon ultrasensitive EQE measurements conducted on active layer thickness dependent organic solar cells, combined with a transfer-matrix model. [42]. Based upon those findings, 


\section{SENSITIVITY OF SUB-GAP EXTERNAL QUANTUM EFFICIENCY MEASUREMENTS}

Armin et al. demonstrated the parasitic influence of low finesse cavity effects on charge transfer state parameterization based upon EQE measurements conducted on active layer thickness dependent organic solar cells. [43] These low finesse cavity effects are also present in perovskite thin-film solar cells, as seen in Fig. 2.6c.
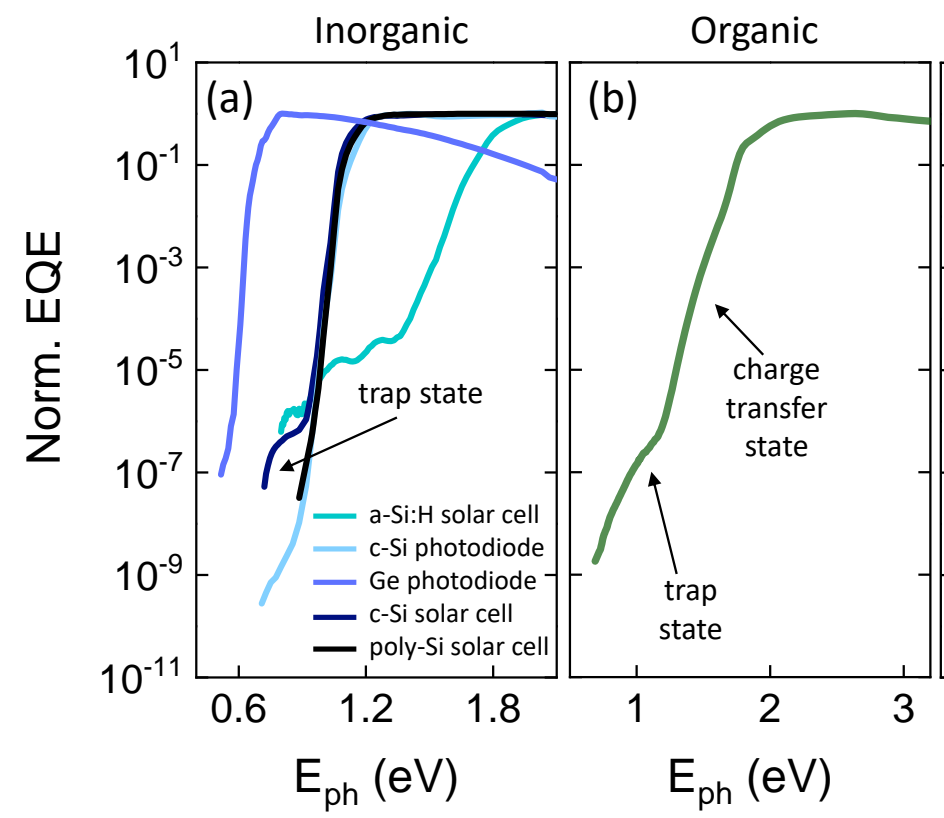

Perovskite

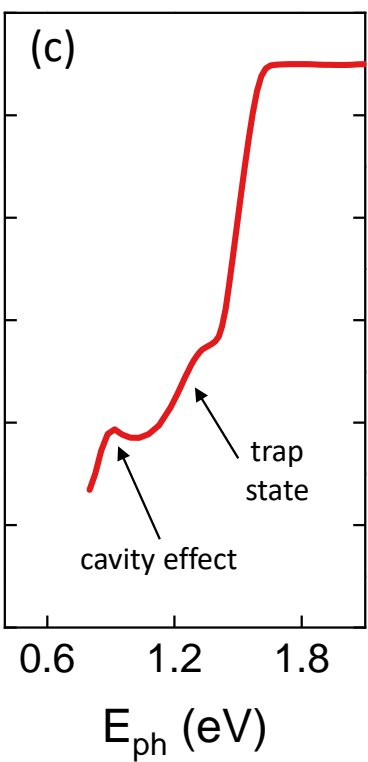

Figure 2.6: Normalized external quantum efficiency [EQE] spectrum of (a) a crystalline silicon [c-Si], poly-crystalline silicon [poly-Si], and hydrogenated amorphous silicon [a-Si:H] solar cell, a silicon and germanium [Ge] photodiode sensor, (b) an organic PCDTBT:PC ${ }_{70} \mathrm{BM}$ solar cell, and (c) a perovskite solar cell plotted as a function of photon energy.

These ultra-sensitive EQE measurements were also key to a series of other collaborative works by the author published in the journals Nature Energy, Physical Review Letters and ACS Energy Letters, in which respectively high-efficiency colloidal $\mathrm{Cs}_{1-\mathrm{x}} \mathrm{Fa}_{\mathrm{x}} \mathrm{PbI}_{3}$ quantum dot solar cells [99], charge carrier transport and generation via trap-mediated optical release in organic semiconductor devices [100], and the relation between exciton emission and voltage losses in PM6:Y6[101] were investigated. These works will not be described in detail in this thesis but show the importance and utility of [ultra-] sensitive EQE measurements. 


\subsection{Conclusion}

In a broader sense, the work detailed in this chapter demonstrated how a relatively simple direct photocurrent methodology can be optimised to reliably measure EQEs as low as $10^{-10}$ with dynamic ranges as high as $100 \mathrm{~dB}$ and to observe sub-gap absorption features at photon energies well below the bandgap in inorganic, organic and perovskite optoelectronic devices. This has considerable utility for probing subtle electro-optical physics and is therefore of great importance for a better understanding of the nature of inter- and intramolecular states and other sub-gap absorption features in organic, inorganic, and perovskite thin-film, optoelectronic devices. [42, 43, 49, 99 101]

A guide to improve the dynamic range (DR) of sensitive external quantum efficiency (EQE) measurements

\section{Box 2.3}

The dynamic range $[\mathrm{DR}]$ of any EQE apparatus depends on three main elements: (i) the light power $L$, (ii) the DUT noise current $I_{\mathrm{NSD}}$, and (iii) the electrical bandwidth $\Delta f$ :

$$
\mathrm{DR}=10 \times \log \left[\frac{q \lambda}{h c} \times \frac{L(\lambda)}{I_{\mathrm{NSD}} \sqrt{\Delta f}}\right]
$$

To increase the DR of an EQE measurement required to detect weak photocurrents [e.g., at photon energies well below an organic semiconductor bandgap], one needs to have:

- a low optical noise light source [i.e., low parasitic stray light, low light intensity instability, and absence of lower harmonics],

- low electrical noise electronic components [i.e., low noise cables, high gain pre-amplifiers with integrated low noise voltage sources, and a Faraday cage for the DUT],

- a high [low] DUT shunt resistance [current] for low thermal noise, and

- small [high] electrical bandwidths [integration times]. 
2. SENSITIVITY OF SUB-GAP EXTERNAL QUANTUM EFFICIENCY MEASUREMENTS 


\section{Chapter 3}

\section{Organic solar cells with near-unity charge generation yield}

The subtle link between charge generation yield [CGY] and bimolecular recombination in organic semiconductor-based photovoltaics is relatively well established as a concept but has proven extremely challenging to demonstrate and probe. Received wisdom teaches that charge generation in excitonic systems will always be lower than non-excitonic semiconductors such as GaAs - but this view is being challenged with the advent of organic semiconductor blends based upon non-fullerene acceptors [NFAs]. This chapter, built-upon the advancement in the sensitivity of the external quantum efficiency measurements described in the previous chapter, presents a newly developed approach to probe the CGY in photovoltaic devices. Near-unity CGY in several model NFA-based systems is observed and measured with unprecedented accuracy. A relatively small increase in yield from 0.984 to 0.993 is found to lead to a reduction in bimolecular recombination from 400 times to 1000 times relative to the Langevin limit. This reduction delivers one of the best thick-junction performance to date in any organic solar cell - notably $16.2 \%$ at $300 \mathrm{~nm}$. These results clearly reveal the relationship between photo-generation and recombination in excitonic semiconductor photovoltaics thus providing a bridge between basic device physics and practical cell engineering. The work presented in this chapter is based upon a collaborative work submitted to the journal Energy \& Environmental Science in 2021.[102] 


\section{ORGANIC SOLAR CELLS WITH NEAR-UNITY CHARGE GENERATION YIELD}

\subsection{Charge generation in non-fullerene acceptor-based or- ganic solar cells}

The performance of organic solar cells [OSCs] has steadily improved in the past two decades but very recently there has been a sharp increase in power conversion efficiencies [PCE]. This has been driven by radical improvements in the constituent materials properties via the development of new non-fullerene electron acceptors [NFAs] - the n-type component. 222] Throughout the history of OSCs, one of the most significant factors limiting the PCE of bulk heterojunction cells [BHJs; the dominant and highest-performing architecture] has been large photocurrent and photovoltage losses. These losses are associated with incomplete charge generation and the excitonic nature of organic semiconductors, as compared to other semiconductors used in photovoltaics such as perovskites, GaAs, and silicon in which charges can be generated with near-unity probability once a photon is absorbed. [103] As described in detail in Chapter 1, Section 1.4, the low dielectric constants of organic semiconductors mean that photo-excitations at room temperature predominantly favor Coulombically bound electron-hole pairs [excitons]. Hence, for excitons to efficiently dissociate, electron donor:acceptor interfaces are required in a BHJ interconnected network. Free charge carriers can be subsequently generated at the interfaces and collected at the corresponding electrodes generating a photocurrent. 104] According to former models based on fullerene-based electron acceptors, efficient charge generation was understood to require a driving force for exciton dissociation, which is generally believed to be provided by the energy difference between the charge transfer [CT] state and the photoexcited exciton [S1] state: $\Delta E_{\mathrm{CT}}=E_{\mathrm{S}_{1}}-E_{\mathrm{CT}_{1}} \cdot[37,105]$ Consequently, the prevailing wisdom is that an energy offset of about $0.3 \mathrm{eV}$ is required to drive the charge genera-

tion process. 106. However, the existence of this driving force also creates a problematic trade-off between the short-circuit current $\left[J_{\mathrm{sc}}\right]$ and open-circuit voltage $\left[V_{\mathrm{oc}}\right]$, which inevitably reduces the maximum PCE for OSCs. 105, 107.

In several recent high-efficiency organic solar cells based on NFAs, in particular the new 'Y6' series, efficient charge generation at low energy offsets has been reported.108 This means that even though the energetic offset between the donor and acceptor is smaller than $0.3 \mathrm{eV}$ [but still larger than $0.1 \mathrm{eV}$ [101, 109]], highly efficient solar cells can be created. Combined with the complementary light absorption of donor and NFA 
semiconductor materials, the smaller energetic offset allows for an increase of $J_{\mathrm{sc}}$ and $V_{\mathrm{oc}}$ simultaneously 110 which ultimately provides for PCEs exceeding $18 \%$. 111 113 These new findings are extremely promising for OSCs technologically and have also encouraged further fundamental work on the dynamics of charge generation. $114-116$ This new insight into NFA-based OSCs [33, 117, 118] has raised important, and potentially field re-defining questions such as: what is the maximum charge generation yield that can be realized in organic semiconductor photovoltaics; is a driving force for efficient charge generation always required; and what role do charge generation kinetics play in determining the charge carrier recombination and device performance?

Even though PCEs as high as $18 \%$ have been realized, $20 \%$ in sight, and a benchmark $25 \%$ optimistically predicted[22], several limitations still hold back OSCs from industrial-scale production with one important limitation being intolerance of the PCE to increasing the thickness of the active layer. As a general rule, OSC performance in most systems is optimized at active layer thicknesses of around $100 \mathrm{~nm}$ - increasing the thickness of the active layer in most cases results in a dramatic loss of fill factor [FFs] and PCE due to relatively poor free carrier transport. However, thin active layer thicknesses are challenging for large-scale production [via high throughput, low-cost methods such as roll-to-roll] and generally also suffer from photocurrent losses due to incomplete above-gap absorption. As such, significant recent efforts have been expended to identify high performance OSC systems that can tolerate thick active layers [i.e., $300 \mathrm{~nm}$ and above].[119, 120] Several such systems have been identified, all of which exhibit a common feature of strongly reduced second order [bimolecular] recombination relative to the Langevin limit. 121 123 In this regard, reduction factors as large as 2000 times have been reported for fullerene-based systems, [119] but they unfortunately suffer from large photovoltage losses and relatively low short-circuit currents. The obvious question arises as to whether any low-offset, low-loss NFA-based system exhibit similarly high reduction factors. To address this question, one must appreciate the subtle relationship between the efficiency of charge photo-generation and bimolecular recombination - as the former approaches unity, the latter should in principle reduce considerably. This important link, although historically appreciated, has been difficult to probe and fully understand, especially under operationally relevant, steady state conditions.

Motivated by these considerations and challenges, in this chapter a study concerning the charge generation quantum yield [CGY] of several NFA-based organic solar cells 


\section{ORGANIC SOLAR CELLS WITH NEAR-UNITY CHARGE GENERATION YIELD}

is described using a new approach involving a kinetic model applied to temperaturedependent, ultra-sensitive external quantum efficiency measurements. The results reveal for the first time, that it is possible to achieve near-unity charge generation quantum yields and realize fully Shockley-type solar cells with organic semiconductors by utilizing state-of-the-art NFA materials. In particular, it is shown that for PM6:BTPeC9, the charge generation efficiency can be as high as 0.993. In addition to the energetics, which have been the subject of several recent and important studies [33, $114-118]$, the central role of kinetics in charge generation is also shown. The results reveal that charge generation in this system involves an energetic barrier which is several times larger than the thermal energy at room temperature [in accordance with previous reports on similar systems [124]] yet is extremely efficient due to the faster dissociation rate of bound states to free charges compared with their decay rate. This means that even though the charge generation mechanism is energetically disadvantaged, it is kinetically driven. 125] The near-unity CGY of PM6:BTP-eC9 results in a reduction of the bimolecular recombination rate constant by more than 1000 times [relative to the Langevin limit] ultimately enabling organic solar cells with active thicknesses of approximately $300 \mathrm{~nm}$ to be realized with a maximum PCE of $16.2 \%$ and FF greater than $71 \%$. The control systems exhibit [considerably] smaller charge generation yields and hence are unable to achieve high efficiencies in the thick-junction limit. The results for PM6:BTP-eC9 solar cells indicate that this system is suitable for lab-to-fab scaling and additionally, the presented approach provides a route for recognizing and developing other systems of such potential. It is also a means to probe in accurate detail the link between charge generation and recombination in excitonic semiconductors. 


\subsection{Energetic offset and device performance}

Four different polymeric donor:NFA systems PM6:Y6, PM6:BTP-eC9, PM6:ITIC, and PBDB-T:EH-IDTBR were employed to study their charge generation yields. Fig. 3.1 a shows the highest occupied molecular orbital [HOMO] and lowest unoccupied molecular orbital [LUMO] energy levels of the four systems PM6:Y6, PM6:BTP-eC9, PM6:ITIC and PBDB-T:EH-IDTBR obtained previously. [108, 112, 126, 127]
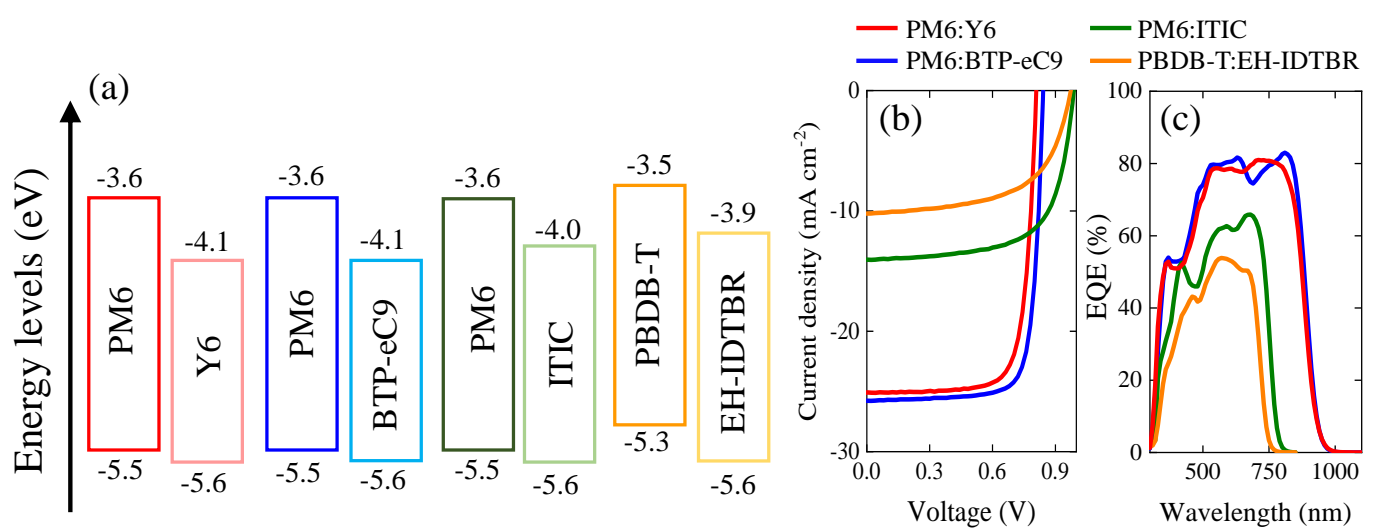

Figure 3.1: (a) Energy levels of polymer donors and non-fullerene acceptors reported from previous work [108, 112, 126, 127], (b) current density versus voltage [J-V] characteristics under artificial 1 sun AM 1.5G conditions, and (c) external quantum efficiency [EQE] of $\sim 100 \mathrm{~nm}$ thick PM6:Y6, PM6:BTP-eC9, PM6:ITIC, and PBDB-T:EH-IDTBR solar cells. Both panels (b) and (c) represent hero devices; the full statistics on multiple devices are provided in the Tab. A.5 - A.8 in Appendix A.

While the former three systems possess rather small HOMO-HOMO energy offsets of roughly $\Delta E_{\text {HOMO-Hомо }} \approx 0.1 \mathrm{eV}$, PBDB-T:EH-IDTBR has a relatively large offset of $\Delta E_{\mathrm{HOMO}}$-номо $\approx 0.3 \mathrm{eV}$. The current density versus voltage $[J-V]$ characteristics along with the photovoltaic external quantum efficiency $\left[\mathrm{EQE}_{\mathrm{PV}}\right]$ spectra of the corresponding optimized OSC BHJ devices are shown in Fig. 3.1 b,c. Experimental details of $J-V$ and EQE measurements are provided in Box 3.1 and Chapter 2; chemical definitions, molecular structures, and a detailed device fabrication are provided in the Appendix A. Despite the low energy offset, PM6:ITIC provides a respectable photovoltaic performance of $\mathrm{PCE}=9.2 \%[9.0 \pm 0.2 \%]$ and maximum $\mathrm{EQE}_{\mathrm{PV}}$ of $66 \%$ [at $\lambda \approx 680 \mathrm{~nm}]$, while superior PCEs of $15.7 \%$ [15.3 $\pm 0.4 \%]$ and $17.1 \%[16.7 \pm 0.2$ \%] were obtained for PM6:Y6 and PM6:BTP-eC9, respectively, both accompanied by 


\section{ORGANIC SOLAR CELLS WITH NEAR-UNITY CHARGE GENERATION YIELD}

$\mathrm{EQE}_{\mathrm{PV}}$ of over $80 \%$ at around $780 \mathrm{~nm}$ excitation wavelength [see Fig. 3.1c], consistent with values published recently. 108, 114 In contrast, PBDB-T:EH-IDBTR achieved a lower PCE of $5.9 \%$ [5.6 $\pm 0.3 \%$ ] and maximum EQE $E_{\mathrm{PV}}$ of $54 \%$ at $570 \mathrm{~nm}$ excitation wavelength. Clearly there is not just a simple correlation between energetic offset and efficiency and so one is led to question as to what drives the relative performance in this small but diverse set of models.

Experimental details of light and dark current density versus applied voltage $(J-V)$ characterization

\section{Box 3.1}

$J-V$ curves under artificial 1 sun AM 1.5G illumination [Oriel LCS- $100^{\mathrm{TM}}$ solar simulator, model 94011A] of the devices [active (pixel) area: $0.04 \mathrm{~cm}^{2}$ ] were measured through a shadow mask with area $0.0256 \mathrm{~cm}^{2}$ [determined with optical microscope] using an Ossila Solar Cell I-V Test System. The solar simulator was calibrated using a silicon reference cell certified by the National Renewable Energy Laboratory [model number $91150 \mathrm{~V}$, series number 2087]. $J-V$ curves were measured in forward direction with $0.5 \mathrm{Vs}^{-1}$ and $0.02 \mathrm{~V}$ step size from $-1 \mathrm{~V}$ to $1.1 \mathrm{~V}$. The approximate values of the series and shunt resistances of devices were (i) directly obtained from the Ossila Solar Cell I-V Test System and (ii) calculated from the inverse of the gradient at the appropriate points of the $J$ - $V$ curve.

Dark $J$ - $V$ curves were measured by a Keithley source-measure unit [model 2400] using an electric-shielded, Faraday cage-like sample holder from Linkam. 


\subsection{Charge generation quantum yield}

To quantify the charge generation quantum yield in the above four systems, a new approach based upon temperature dependent, ultra-sensitive $\mathrm{EQE}_{\mathrm{PV}}$ measurements [see Fig. 3.2] was employed. In general, the EQE is given by

$$
\mathrm{EQE}_{\mathrm{PV}}=\eta_{\mathrm{abs}}(T) \times \mathrm{IQE}(T),
$$

noting that the absorption probability, respectively efficiency, of the active layer $\eta_{\mathrm{abs}}$ is temperature dependent [see Box 3.2]. Here, $\operatorname{IQE}(T)$ denotes the internal quantum efficiency and is related to the charge generation yield via

$$
\mathrm{IQE}(\mathrm{T})=\eta_{\mathrm{CC}} \times \mathrm{CGY}(\mathrm{T}),
$$

where $\eta_{\mathrm{CC}}$ is the charge collection efficiency. Since charge carrier recombination is expected to be small due to the low light intensities [at which the EQEs were probed], changes in $\eta_{\mathrm{CC}}$ with temperature were assumed to be negligible. Hence, to probe charge generation, the normalized $\operatorname{IQE}(T)$ is used, which is given by

$$
\operatorname{IQE}^{*}(T)=\frac{\operatorname{IQE}(T)}{\operatorname{IQE}\left(T_{\max }\right)}=\frac{\mathrm{EQE}_{\mathrm{PV}}(T)}{\operatorname{EQE}_{\mathrm{PV}}\left(T_{\max }\right)} \times\left[\frac{\eta_{\mathrm{abs}}(T)}{\eta_{\mathrm{abs}}\left(T_{\max }\right)}\right]^{-1} .
$$

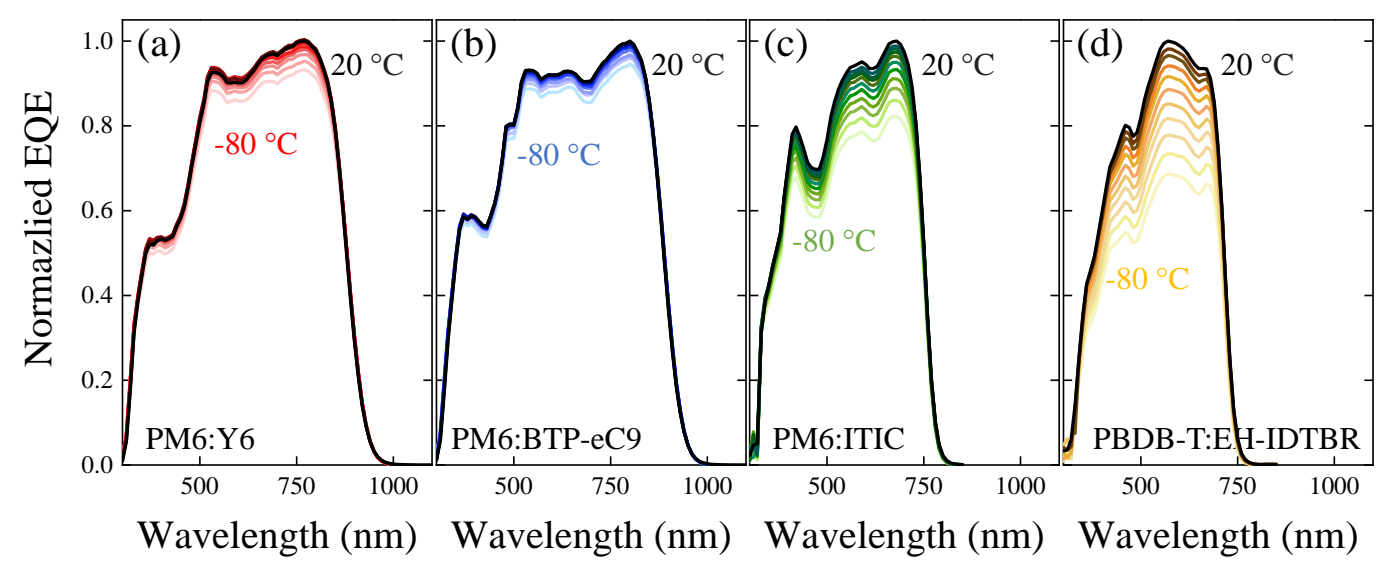

Figure 3.2: Temperature dependent external quantum efficiency [EQE] spectra at zero applied bias voltage [short-circuit] of [a] PM6:Y6, [b] PM6:BTP-eC9, [c] PM6:ITIC, and [d] PBDB-T:EH-IDTBR. 


\section{ORGANIC SOLAR CELLS WITH NEAR-UNITY CHARGE GENERATION YIELD}

In Fig. 3.3 a, the logarithm of IQE* of the four performance-optimized NFA-based systems at an excitation wavelength of $\lambda_{\text {exc }}=650 \mathrm{~nm}$ are shown as a function of the inverse thermal energy $\left[k_{\mathrm{B}} \mathrm{T}\right]^{-1}$ [with $k_{\mathrm{B}}$ being the Boltzmann constant]. The experimental data [symbols] are fitted to a kinetic model [solid lines] which accounts for the competition between thermally-activated dissociation [of rate constant $k_{\mathrm{d}}(T)$ ] and recombination [with rate constant $k_{\mathrm{f}}$ ] of bound electron-hole pairs:

$$
\operatorname{CGY}(T)=\left[1+\frac{k_{\mathrm{f}}}{k_{\mathrm{d}}(T)}\right]^{-1}, 128
$$

with

$$
k_{\mathrm{d}}(T)=k_{0} \times \exp \left[-\frac{E_{\mathrm{a}}}{k_{\mathrm{B}} T}\right],
$$

where $k_{0}$ corresponds to the rate $k_{\mathrm{d}}$ at infinite temperature and $E_{\mathrm{a}}$ is the activation energy of the charge dissociation.

\section{Experimental details of temperature dependent absorptance measurements}

\section{Box 3.2}

A liquid nitrogen-based, temperature-controlled sample holder from Linkam in combination with a high-performance spectrophotometer Lambda950 from PerkinElmer and the integrating sphere module are used to measure temperature dependent absorptance of films on glass. Relative changes in absorptance [see Eq. 3.3; last term] are calculated by normalizing the absorptance spectra to the maximum temperature. Temperature dependent absorptance spectra of PM6:Y6, PM6:BTP-eC9, PM6:ITIC, and PBDB-T:EH-IDTBR are provided in Fig. B.2 in Appendix A.

From the fits, the activation energies of PM6:BTP-eC9 $\left[E_{\mathrm{a}}=118 \pm 10 \mathrm{meV}\right], \mathrm{PM} 6: \mathrm{Y} 6$ $\left[E_{\mathrm{a}}=103 \pm 6 \mathrm{meV}\right]$, PBDB-T:EH-IDTBR $\left[E_{\mathrm{a}}=45 \pm 2 \mathrm{meV}\right]$ and PM6:ITIC $\left[E_{\mathrm{a}}=53\right.$ $\pm 4 \mathrm{meV}$ ] were determined [see Fig. 3.3 b]. Based on the analysis, all four BHJ systems exhibit a small, yet non-negligible energy barrier for charge generation, strongly suggesting these systems possess a kinetically driven CT state dissociation and thermally activated $k_{\mathrm{d}}$ rate at room temperature [RT] [i.e., $E_{\mathrm{a}}>25 \mathrm{meV}$ ]. The corresponding 
CGYs of the four OSCs at RT are shown in Fig. 3.3c. Relatively low CGYs of CGY $=0.633 \pm 0.026$ for PBDB-T:EH-IDTBR and CGY $=0.857 \pm 0.019$ for PM6:ITIC were obtained, consistent with their quite low $\mathrm{EQE}_{\mathrm{PV}}$ of approximately $50 \%$ and 64 $\%$ at $650 \mathrm{~nm}$ [open symbols in Fig. 3.3c], respectively. Interestingly, while PM6:Y6 shows a very high charge generation yield of CGY $=0.984 \pm 0.003$ close to unity, this value increases even further to $\mathrm{CGY}=0.993 \pm 0.003$ for the PM6:Y6 derivative PM6:BTP-eC9.
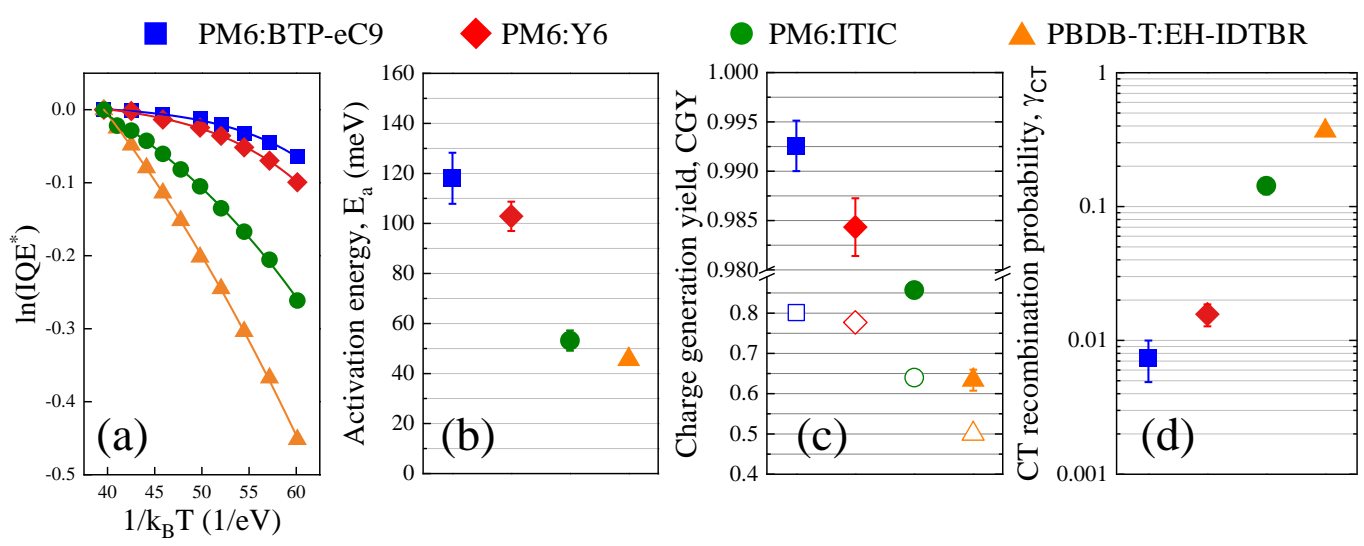

Figure 3.3: (a) Logarithm of the normalized internal quantum efficiency [IQE*] of $\sim 100$ nm thick PM6:BTP-eC9, PM6:Y6, PM6:ITIC and PBDB-T:EH-IDTBR solar cells. The experimental data are indicated by the symbols, while solid lines show the corresponding fits to the kinetic model of the charge generation yield [CGY]. The excitation wavelength was set to $650 \mathrm{~nm}$ and no bias voltage was applied. (b) The extracted activation energy $\left[E_{\mathrm{a}}\right]$, (c) charge generation yield [CGY] [filled symbols], and (d) CT recombination probability $\left[\gamma_{\mathrm{CT}}\right]$ for PM6:BTP-eC9, PM6:Y6, PBDB-T:EH-IDTBR and PM6:ITIC based on the fits. For comparison, the $\mathrm{EQE}_{\mathrm{PV}}$ [open symbols] at RT and $650 \mathrm{~nm}$ are included in panel (c).

This new approach combining ultra-sensitive, temperature dependent and absorptancecorrected EQE measurements with a kinetic rate model, allows one to not only evaluate the CGY with very high accuracy, but also proves that near-unity charge generation quantum yields in excitonic organic solar cells are indeed possible. Although all four OSC systems exhibit similar activation energies, a near-unity CGY was only obtained in PM6:Y6 and PM6:BTP-eC9 suggesting different CT state recombination kinetics. The corresponding recombination probability for $\mathrm{CT}$ states

$$
\gamma_{\mathrm{CT}}=1-\mathrm{CGY}
$$




\section{ORGANIC SOLAR CELLS WITH NEAR-UNITY CHARGE GENERATION YIELD}

critically determined by the rate constant ratio $k_{\mathrm{f}} / k_{\mathrm{d}}$, is shown in Fig. 3.3d at RT. Indeed, while the PBDB-T:EH-IDTBR and PM6:ITIC devices showed a relatively high $\gamma_{\mathrm{CT}}$ of $0.37 \pm 0.03$ and $0.14 \pm 0.02$, respectively, a $\gamma_{\mathrm{CT}}=0.016 \pm 0.03$ for PM6:Y6 and an approximately two times smaller $\gamma_{\mathrm{CT}}=0.007 \pm 0.003$ for PM6:BTP-eC9 were found. The above findings demonstrate that both energetics and kinetics determine the CGY with the latter being the key factor underpinning the better device performance in PM6:BTP-eC9 and PM6:Y6. The low CT state recombination probabilities have direct implications for the bimolecular recombination coefficient $\beta$ in PM6:Y6 and PM6:BTPeC9. In this regard, the important figure-of-merit is the Langevin reduction factor

$$
\gamma=\frac{\beta}{\beta_{\mathrm{L}}}
$$

where $\beta_{\mathrm{L}}$ is the Langevin recombination coefficient given by

$$
\beta_{\mathrm{L}}=q\left[\frac{\mu_{\mathrm{n}}+\mu_{\mathrm{p}}}{\varepsilon \varepsilon_{0}}\right]
$$

with $q$ being the elementary charge, $\mu_{\mathrm{n}}\left[\mu_{\mathrm{p}}\right]$ the electron [hole] mobility, and $\varepsilon$ the permittivity of the active layer [see Chapter 1, Section 1.8.1]. However, the CGY is related to the Langevin-reduction factor via

$$
\gamma=\gamma_{\mathrm{CT}} \times \gamma_{\text {geo }}
$$

where

$$
\gamma_{\text {geo }}=\frac{\beta_{\text {enc }}}{\beta_{\mathrm{L}}}
$$

is the geometrical reduction factor with $\beta_{\text {enc }}$ being the encounter rate coefficient for charge carriers to form CT states. [129] Therefore, significantly reduced Langevin reduction factors in these two systems are expected. 


\subsection{On the origin of strongly suppressed recombination}

To verify the findings described in Section 3.3, charge transport and recombination measurements were conducted to estimate $\gamma$. Firstly, resistance-dependent photovoltage [RPV, see Box 3.3 measurements at zero applied bias voltage were applied to obtain the mobilities in PM6:Y6 and PM6:BTP-eC9 operational devices [see Fig. 3.5]. For PM6:Y6, slower and faster charge carrier mobilities of $\mu_{\text {slow }} \approx 2 \times 10^{-4} \mathrm{~cm}^{2} \mathrm{~V}^{-1} \mathrm{~s}^{-1}$ and $\mu_{\text {fast }} \approx 1.2 \times 10^{-3} \mathrm{~cm}^{2} \mathrm{~V}^{-1} \mathrm{~s}^{-1}$ were extracted, respectively, whereas $\mu_{\text {slow }} \approx 4 \times 10^{-4}$ $\mathrm{cm}^{2} \mathrm{~V}^{-1} \mathrm{~s}^{-1}$ and $\mu_{\text {fast }} \approx 2 \times 10^{-3} \mathrm{~cm}^{2} \mathrm{~V}^{-1} \mathrm{~s}^{-1}$ were found in PM6:BTP-eC9.

Experimental details of resistance-dependent photovoltage (RPV) measurements

\section{Box 3.3}

For resistance-dependent photovoltage measurements, the device under test [DUT] with active layer thickness $d$ is in series with a variable load resistance, $R_{\text {load }}$ [see Fig 3.4; upper panel]. A diode pumped, Q-switched Nd:YAG laser [Viron, Quantel Laser] is used to create a short laser pulse, thus photo-generating charge carriers in the active layer of the DUT. An oscilloscope [Rohde \& Schwarz, RTM 3004], in parallel to the DUT, is used to measure the photo-pulse induced photovoltage of the DUT. The load resistance is stepwise increased from $50 \Omega$ to $1 \mathrm{M} \Omega$.

From the RPV transients and the corresponding transit times $\left[t_{\mathrm{tr}}\right]$ [see Fig 3.4; lower panel] the charge carrier mobility is calculated via

$$
\mu=\frac{d^{2}}{t_{\mathrm{tr}} \times\left|V-V_{\mathrm{bi}}\right|},
$$

where $V_{\mathrm{bi}}$ denotes the built-in voltage, and $V$ corresponds to the applied bias voltage. 1102, 119, 130, 131]

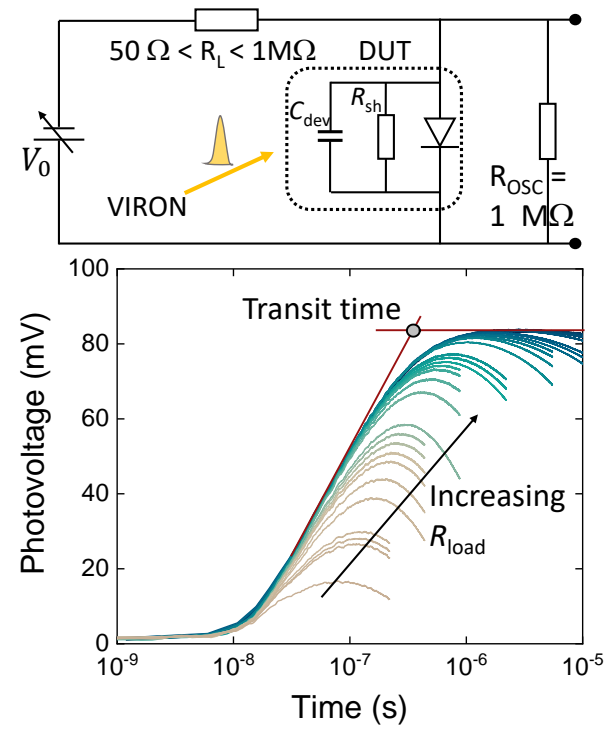

Figure 3.4: Simplified schematic of the RPV setup [upper panel] and RPV transients for transit time determination [lower panel]. 


\section{ORGANIC SOLAR CELLS WITH NEAR-UNITY CHARGE GENERATION YIELD}

Note that the mobility values obtained for PM6:Y6 are close to the ones estimated via space-charge limited current [SCLC] measurements [see Box 6.3] by Shoaee and co-workers. 132, 133. Subsequently, electrons [holes] were assigned to the faster [slower] carriers.

Next, the corresponding $\beta$ was quantified from steadystate double injection [DoI] currents [see Box 3.4]. Based on the DoI measurements, $\gamma \approx 2.5 \times$ $10^{-3}$ for PM6:Y6 and $\gamma \approx$ $1.0 \times 10^{-3}$ for PM6:BTPeC9 were found, respectively, corresponding to 400 times and 1000 times reduced recombination relative to the Langevin limit. This confirms the presence of strongly reduced recombination coefficients in PM6:Y6 and PM6:BTPeC9, as expected from the near-unity CGY. From the

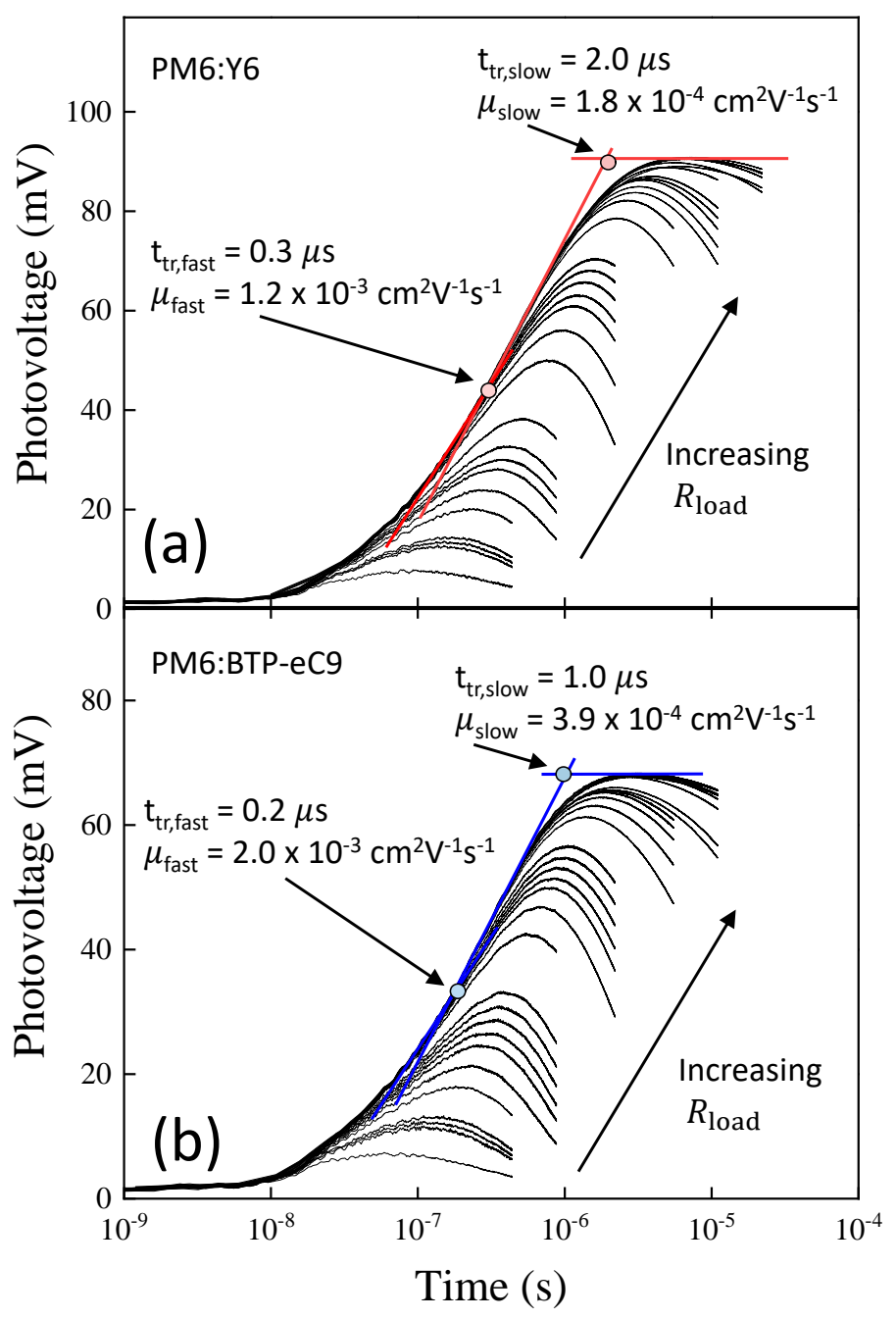

Figure 3.5: Resistance-dependent photovoltage [RPV] transients of a (a) $200 \mathrm{~nm}$ thick PM6:Y6 and a (b) 210 $\mathrm{nm}$ thick PM6:BTP-eC9 device. The laser excitation wavelength was set to $525 \mathrm{~nm}$, and no bias voltage was applied [i.e., short-circuit]. The fast and slow carrier mobilities are quantified from their corresponding transit times $\left[t_{\mathrm{tr}}\right]$ shown in panel (a) and (b) assuming built-in voltages of $V_{\mathrm{bi}}=1.1$ V for both PM6:Y6 and PM6:BTP-eC9. Experimental details of the RPV setup are provided in Box 3.3.

Langevin-reduction factor $\gamma$ and the CT state recombination probability $\gamma_{\mathrm{CT}}$, the geometrical Langevin-reduction factors were estimated to $\gamma_{\text {geo }} \approx 0.16$ and $\gamma_{\text {geo }} \approx 0.13$ for PM6:Y6 and PM6:BTP-eC9, respectively. These values are consistent with theoretically expected values for $\gamma_{\text {geo }}$, [77] verifying the accuracy of the obtained CGY values. 
Experimental details of (dark) steady-state double-injection (DoI) current measurements

\section{Box 3.4}

The space-charge-limited current [of electrons and holes], $J_{\mathrm{sc}}$, in the forward-bias $\left[V \gg V_{\mathrm{bi}}\right]$ is limited by the recombination of electrons and holes injected from the contacts [here, $V$ is the applied voltage and $V_{\mathrm{bi}}$ the built-in voltage]. For $\beta \gg \beta_{\mathrm{L}}$ [with $\beta$ being the bimolecular recombination coefficient and $\beta_{\mathrm{L}}$ denoting the Langevin coefficient, see Eq. 3.8], the injected electrons and holes from the electrodes recombine as soon as they encounter each other in the active layer. In this limit, the total current $J$ is given by the combined space-charge limited currents from the separate electron- and hole-dominated regions:

$$
J_{\mathrm{SCL}}=\frac{9}{8} \varepsilon \varepsilon_{0}\left(\mu_{\mathrm{n}}+\mu_{\mathrm{p}}\right) \frac{V_{\mathrm{dev}}^{2}}{d^{3}} .
$$

Here, $V_{\mathrm{dev}}$ is the applied voltage across the device and $d$ is the thickness of the active layer. For $\beta \ll \beta_{\mathrm{L}}$, corresponding to the case when the recombination is reduced with respect to the Langevin rate, the electron and hole currents do not immediately annihilate each other upon meeting in space, resulting in the establishment of an injected electron-hole plasma in the bulk. In this case, the total current $J$ is given by:

$$
J_{\mathrm{DI}}=\frac{9}{8} \varepsilon \varepsilon_{0}\left(\mu_{\mathrm{eff}}\right) \frac{V_{\mathrm{dev}}^{2}}{d^{3}},
$$

where

$$
\mu_{\mathrm{eff}}=\frac{2}{3} \sqrt{\frac{4 \pi \mu_{\mathrm{n}} \mu_{\mathrm{p}}}{\gamma}} .
$$

Finally, the externally applied voltage $V$ is related to the device voltage via

$$
V_{\mathrm{dev}}=V-J R_{\text {series }} \times A,
$$

where $R_{\text {series }}$ is the external series resistance and $A$ is the device area. Neglecting the presence of an external series resistance [especially in thin devices] generally results in an underestimation of $\gamma$. Note that the DoI theory is only valid for $V \gg V_{\mathrm{bi}}$; therefore, to account for the presence of a built-in voltage, $V$ is usually replaced by $V-V_{\mathrm{bi}}$ in the above relations for $J_{\mathrm{SCL}}$ and $J_{\mathrm{DI}}$. 


\section{ORGANIC SOLAR CELLS WITH NEAR-UNITY CHARGE GENERATION YIELD}

\subsection{From thin- to thick-junction organic solar cells}

The strongly suppressed recombination [low $\gamma$, being a direct consequence of the nearunity CGY, is expected to translate into improved device performance in thick junctions. Concomitantly, PM6:Y6, PM6:BTP-eC9, PM6:ITIC and PBDB-T:EH-IDTBR devices were fabricated with different active layer thickness from 30 to $470 \mathrm{~nm}$. The $J-V$ curves of all four systems are shown in Fig. 3.6 .

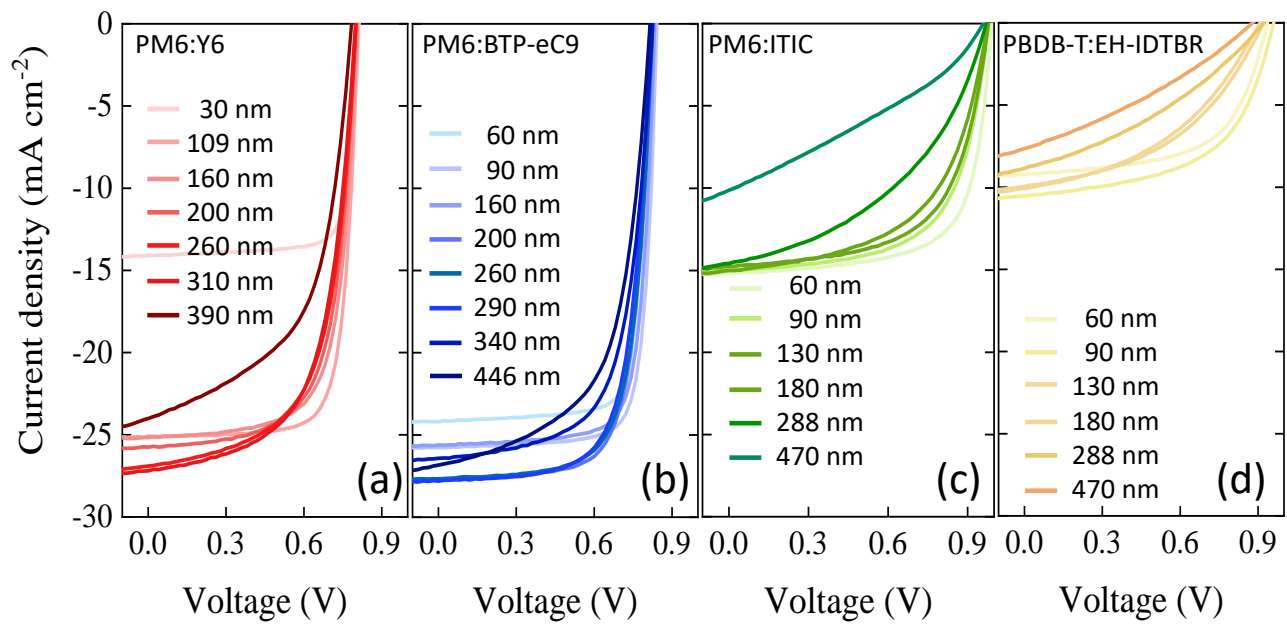

Figure 3.6: Current density versus applied voltage characteristics of (a) PM6:Y6, (b) PM6:BTP-eC9, (c) PM6:ITIC, and (d) PBDB-T:EH-IDTBR solar cells under artificial 1 sun AM 1.5G illumination. Photovoltaic parameters are provided in Tab. A.5-A.8 in Appendix A.

Fig. 3.7 a,b show the corresponding FF and PCE at different active layer thicknesses. The PCE of PBDB-T:EH-IDTBR and PM6:ITIC systems drop off rapidly with increasing active layer thickness due to a significant reduction of both $J_{\mathrm{sc}}$ and FF. In contrast, PM6:Y6 shows a much less severe thickness dependency, with $\mathrm{FF}=61.4 \%$, $J_{\mathrm{sc}}=26.8 \mathrm{~mA} \mathrm{~cm}^{-2}$ and $\mathrm{PCE}=13.1 \%$ for the hero device at a thickness of $300 \pm$ $10 \mathrm{~nm}$, confirming the relation between CGY and efficiency of thick-junction OSCs. However, although PM6:Y6 exhibits respectable device performance in thick junctions, a substantial degradation in efficiency is still observed when compared with the $100 \mathrm{~nm}$ thick junction. Finally, the best thickness dependent device performance is obtained for PM6:BTP-eC9 showing a hero PCE of $16.2 \%$, together with an FF of $71.3 \%$, in a $300 \pm 10 \mathrm{~nm}$ thick junction. At the time of writing, this $\sim 300 \mathrm{~nm}$ thick PM6:BTP- 
eC9 BHJ is one of the most efficient binary OSC at this active layer thickness, leaving PM6:BTP-eC9 as a promising candidate for lab-to-fab up-scaling. To further

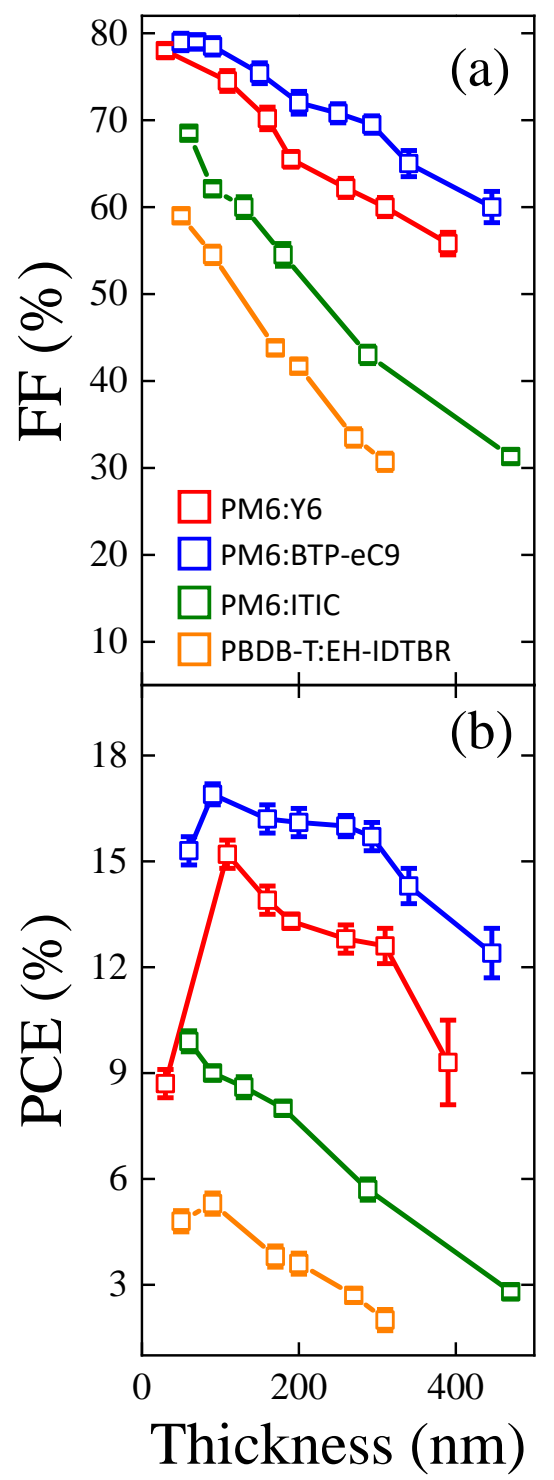

Figure 3.7: (a) Averaged fill factors $[\mathrm{FFs}]$ and $(\mathbf{b})$ power conversion efficiencies [PCEs] of PM6:Y6, PM6:BTP-eC9, PM6:ITIC, and PBDB-T:EH-IDTBR OSCs plotted as a function of active layer thickness. Error bars represent standard deviations. confirm that the thickness-dependent device performance is related to the suppressed recombination [and thus the CGY], electro-optical device simulations for PM6:Y6 and PM6:BTP-eC9 devices were performed, based on a drift-diffusion [DD] model. 80, 81] An optical transfer-matrix model which accounts for optical interference effects in the device stack was used to calculate the generation profile within the active layer. 134, 135] Refractive indices of PM6:Y6 and PM6:BTP-eC9 [see Fig. B.3 in Appendix B were used as input for the optical model. The generated 'charge generation' profiles under AM 1.5G condition were then imported to the DD model to calculate the corresponding $J-V$ parameters. In

Fig. 3.8, the experimentally measured [symbols] and simulated [solid lines] PV parameters are plotted as a function of active layer thickness. A good overall agreement between simulations and experimental data is obtained, validating the measured transport and recombination parameters. It is worth noting that a considerable deviation between experimental and simulation data is only seen for the thickest PM6:Y6 device [see Fig. 3.8a,b]. A possible explanation for this deviation could be the presence of trap states[49], which are expected to become increasing important at larger thicknesses, [136] or morphological non-uniformities due to different post-deposition active layer drying rates. 


\section{ORGANIC SOLAR CELLS WITH NEAR-UNITY CHARGE GENERATION YIELD}

Importantly, the enhanced performance of $\mathrm{PM} 6: \mathrm{BTP}-\mathrm{eC} 9$, relative to $\mathrm{PM6:Y6}$, is well-reproduced by the DD model confirming the lower recombination coefficient in PM6:BTP-eC9 to be the primary underlying reason for the relative improvement. Since $\gamma \sim 1-$ CGY, the value of the Langevin reduction factor is very sensitive to small changes of CGY in systems where CGY $\approx 1$. This also explains why the modest increase of only 0.9 \% in the CGY in PM6:BTP-eC9, relative to PM6:Y6, can result in noticeably smaller $\gamma$ and subsequently considerably improved device performance in thicker junctions.

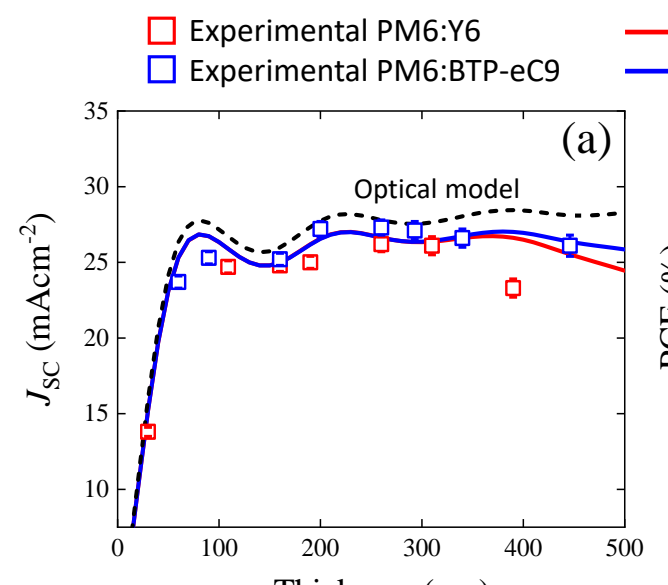

Electro-optical simulated PM6:Y6

- Electro-optical simulated PM6:BTP-eC9
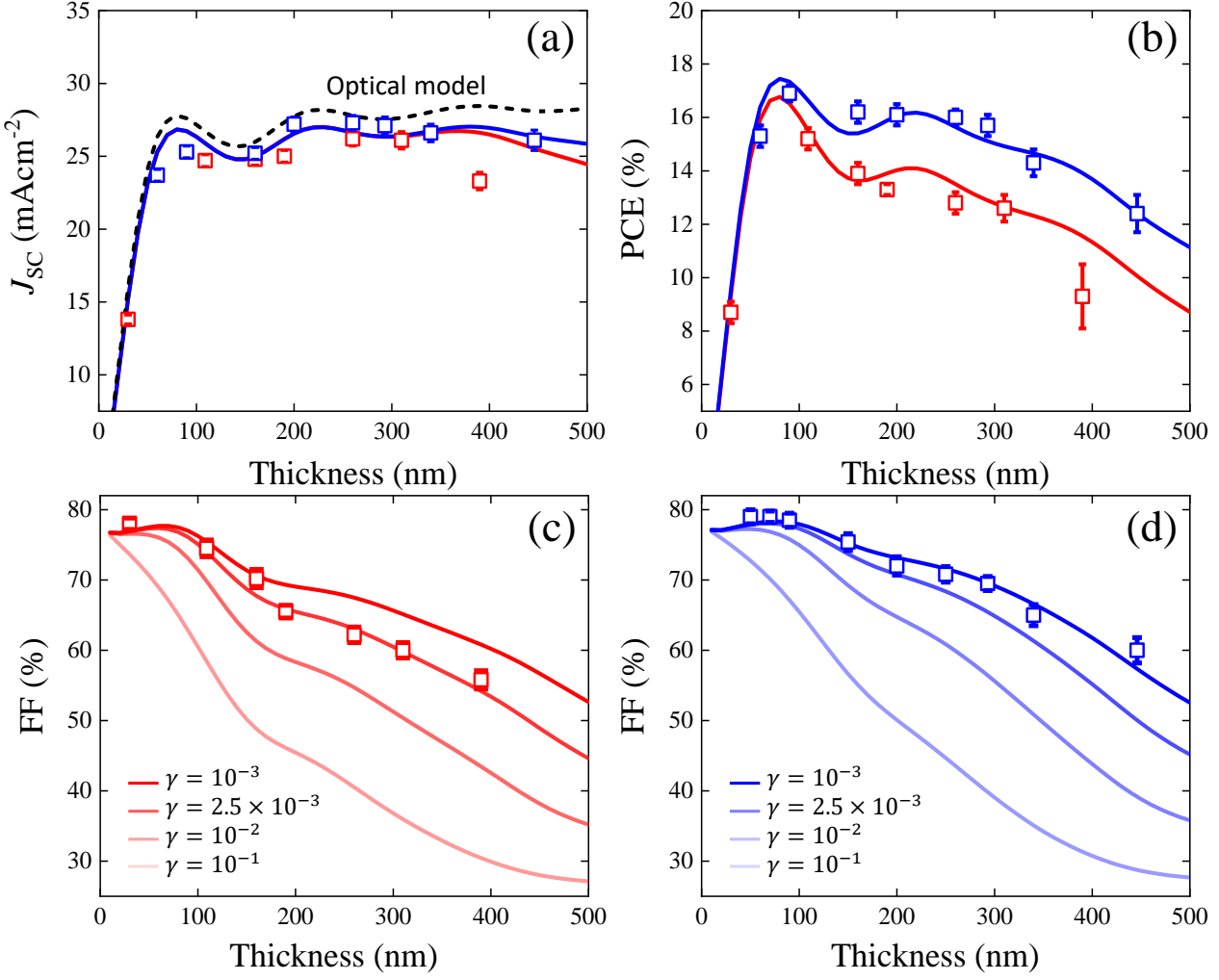

Figure 3.8: (a) Short-circuit current density $\left[J_{\mathrm{sc}}\right]$ and (b) power conversion efficiency [PCE] of PM6:Y6 and PM6:BTP-eC9 plotted as a function of active layer thickness. The symbols are experimental data [averages with standard deviations as error bars], solid [dashed] lines correspond to drift-diffusion [optical transfer-matrix model] simulations. (c) Experimentally obtained fill factors [FFs] [symbols] of PM6:Y6 plotted as a function of active layer thickness and compared with DD simulations [solid lines] assuming different Langevin reduction factors. (d) Repetition of panel (c) but for PM6:BTP-eC9 OSCs. The input parameters for the simulations are provided in Tab. A.4 in Appendix A; thickness dependent EQE spectra are shown in Fig. B.4 in Appendix B. 
For reference, the FF of PM6:Y6 and PM6:BTP-eC9 devices with different $\gamma$ [but keeping the mobilities the same as before] were also simulated and are shown in Fig. 3.8c,d. It is clear that both systems benefit from reduced recombination enabling high efficiencies in thick junctions, whereas the degradation of the FF at thicker junctions is much more pronounced for larger recombination coefficients. It is worth noting, however, that a sufficiently high electron mobility is also vital to ensure balanced charge extraction in conventional thick OSC junctions [where charge carriers are predominantly generated near the transparent electrode], as discussed in previous works. [40, 137] 


\section{ORGANIC SOLAR CELLS WITH NEAR-UNITY CHARGE GENERATION YIELD}

\subsection{Organic solar cells with Shockley-type behaviour}

Finally, the competition between charge extraction and recombination in PM6:Y6 and PM6:BTP-eC9 devices at different thicknesses were quantified using the modified Shockley model proposed by Neher et al. 139 The associated figure-of-merit $\alpha_{\mathrm{FOM}}$ is given by:

$$
\alpha_{\mathrm{FOM}}^{2}=\frac{q \beta J_{\mathrm{G}} d^{3}}{4 \mu_{\mathrm{n}} \mu_{\mathrm{p}}\left(k_{\mathrm{B}} T\right)^{2}},
$$

where $J_{\mathrm{G}}$ is the photogeneration current density and $d$ is the active layer thickness. In this context, if $\alpha_{\mathrm{FOM}}<1$, the device is not limited by transport and thought to be Shockley-type. In Fig. 3.9 a, the FFs of PM6:Y6 and PM6:BTP-eC9 at different thicknesses are shown as a function of $\alpha_{\text {FOM }}$ estimated from the measured charge transport and recombination parameters. Both systems exhibit with increasing active layer thickness a regime of Shockley-type behavior. This behavior is maintained up to active layer thicknesses of $109 \mathrm{~nm}$ and $200 \mathrm{~nm}$ for PM6:Y6 and PM6:BTP-eC9, respectively. For comparison, in Fig. 3.9a the analytical approximation for the $\mathrm{FF}$ as per the modified Shockley model [dashed line] is also included. [139] To further support the presence of Shockley-type behavior, the effective series resistance $\left[R_{\mathrm{S} \text {,eff }}\right]$ is calculated from the inverse slope of the light $J-V$ 
curve at $V=V_{\text {oc }}$, for cells of different junction thicknesses. Based on the modified Shockley equation [139], $R_{\mathrm{S}, \mathrm{eff}}=R_{\mathrm{S}}+k T\left[q J_{\mathrm{G}}\right]^{-1} \times\left[1+\alpha_{\mathrm{FOM}}\right]$ is found, where $R_{\mathrm{S}}$ is the combined series resistance from the contacts, the electrodes, and the external wires. As shown in Fig. 3.9b, upon comparing $R_{\mathrm{S}, \text { eff }}$ from experimental data [symbols] with the analytical $R_{\mathrm{S} \text {,eff }}$ using the estimated $\alpha_{\mathrm{FOM}}$ [solid lines], a good overall agreement was indeed obtained.

From the above combined experimental and simulation results it is clear that the CGY of a thin-film OSC is linked to both donor:acceptor interfacial energetics [e.g., energy offset and activation energy] and kinetics, inevitably reflected in the efficiency of the corresponding thick junction BHJ. Based on this framework it becomes clear that a $0.9 \%$ higher, near-unity CGY, translating into a $\sim 2.5$ stronger suppressed recombination, leads to a drastic improvement in PM6:BTP-eC9 relative to PM6:Y6 not only in thin junction BHJs, but more importantly in the thick-junction limit. This subtle link between photo-generation and charge recombination provides a powerful tool to merge basic device physics and practical solar cell engineering, allowing for promising candidates for industrial large-area solar cell fabrication to be identified. 


\section{ORGANIC SOLAR CELLS WITH NEAR-UNITY CHARGE GENERATION YIELD}

\subsection{Conclusion}

The work detailed in this chapter focused on the charge generation dynamics of several state-of-art organic solar cells, where a new kinetic was model, based upon temperature dependent ultra-sensitive external quantum efficiency measurements described in the previous chapter, was introduced. Notably, near-unity charge generation quantum yields [0.993] were found in a recently reported high efficiency PM6:BTP-eC9 system, outperforming its PM6:Y6 [CGY = 0.984] counterpart and other non-fullerene solar cell models. This apparently small difference in the CGY makes these two systems very different in how they perform in the industrially-relevant thick-junction limit. The work described in this chapter not only confirmed it is possible to achieve nearunity charge generation quantum yields using organic semiconductors and to realize Shockley-type solar cells [i.e., not limited by charge transport], but also showed the importance of kinetics in charge generation in addition to the energetics. More significantly, PM6:BTP-eC9 was found to exhibit a faster dissociation rate of bound states to free charges and slower decay rate compared to PM6:Y6, enabling a reduction of bimolecular recombination rate constant by more than 1000 times. As a result, an unprecedented power conversion efficiency over $16 \%$ with an FF greater than $71 \%$ was achieved in PM6:BTP-eC9 solar cell with an active layer thicknesses of approximately $300 \mathrm{~nm}$. These results not only reveal the interplay between charge generation, recombination, and device efficiency in novel state-of-art non-fullerene solar cells, but also present a route for recognizing and developing other systems with similar potential. 


\section{Chapter 4}

\section{Low intensity integral time-of-flight technique}

The generation of free charges in light-harvesting devices is a multi-step process, which can be challenging to probe due to the complexity of contributed energetic states and the competitive character of different mechanisms driving the generation process. In this chapter, a new technique is described to probe the charge generation in thinfilm solar cells. The technique combines capacitance measurements with the integralmode time-of-flight method extended to the low intensity regime [LIITOF], and allows the sensitive measurement of photogenerated charge carrier densities. The theoretical framework of the method is verified by drift-diffusion simulations, and the applicability of LIITOF is demonstrated for organic semiconductor and perovskite thin-film solar cells. Further, the field dependence of the charge generation efficiency is examined in detail and the LIITOF results are correlated with those obtained via time delayed collection field conducted on the same devices.

The work presented in this chapter is based on a collaborative work of the author currently under preparation for submission. 


\section{LOW INTENSITY INTEGRAL TIME-OF-FLIGHT TECHNIQUE}

\subsection{Debates on charge generation pathways}

As discussed in previous chapters, neat organic semiconducting materials are characterized by incomplete free charge generation [at room temperature] which is directly related to their excitonic nature by a virtue of their low permittivity. The involved mechanism of charge transfer $[\mathrm{CT}]$ state dissociation into free charge carriers is hereby not well understood. While the work of Braun[68] suggested that CT dissociation in organic solar cells [OSCs] is field dependent [see Chapter 1, Section 1.7.2, most efficient donor: acceptor blends show either no or only week dependence on the electric field. [66, 140-142] Hence, more advanced models have been proposed to explain the fast and efficient dissociation of CT states to free charges. Clarke and Durrant, for instance, considered the role of entropy in CT dissociation events[27], while other models included the role of energetic disorder [118], delocalization[118, 143], and vibronically excited [i.e., 'hot'] states[144] in the formation of free, separated charges. The role of 'hot CT states' was challenged by Kurpiers and co-workers, who found the electric field and temperature dependent charge generation in fullerene acceptor [FA] based organic bulk-heterojunctions [BHJs] to be independent of excess energy. [66] They concluded, in line with past findings by Vandewal et al. [61], that charge generation proceeds through thermalized $\mathrm{CT}$ states independent of activation energies and the energetic offset between relaxed singlet excited states $\mathrm{S}_{1}$ and $\mathrm{CT}$ states. This is also to be expected in the new class of state-of-the-art BHJs based non-fullerene acceptors [NFA] exhibiting low energetic offsets. Despite this, recent studies on the CT dissociation conducted on NFA organic solar cells suggested an electric field and excess energy dependent charge generation. 145] Furthermore, Karuthedath and co-workers proposed a model based on interfacial D: A band-bending inducing quadrupole moments suggesting the requirement of an ionization energy offset to drive charge generation in FA and NFA OSCs sufficiently. [146, 147]

To gain more insight into the process of CT state dissociation, methods capable of probing free charge generation efficiency in thin-film solar cells independent of bulk recombination are needed. This has proven to be challenging, but if successful, it can be a guide towards a better understanding of the mechanism of charge generation in stateof-the-art OSCs. In the past, several measurement techniques have been employed to investigate free charge generation in optoelectronic devices. While intensity dependent 
photocurrent [IPC] [148] and external [internal] quantum efficiency [EQE [IQE] [29, 41] are prominent examples of steady-state techniques, transient absorption spectroscopy [TAS] 149 151] and time-delayed collection field [TDCF] are, in turn, commonly used time-resolved probing techniques. Probing charge generation using IPC is questionable as the results can be affected by first order losses due to trap-assisted recombination and so-called pseudo-first order recombination near the electrodes. [152, 153] TAS, in turn, has been used to probe free charge generation via detecting geminate recombination at early time scales. [154, 155] However this method is not applicable to high efficiency systems where no geminate losses can be detected. TDCF has been the most useful method and frequently used to study the free charge generation dynamics in organic and perovskite solar cells. [66, 156, 157] However, while TDCF remains a powerful methodology, it uses a complex circuit requiring special current pre-amplifiers with fast bias ramp-up times, and suffers from $R C$-time limitations at short time-scales.

In this chapter an alternative, easy-to-use measurement technique is advanced to probe charge generation in optoelectronic devices. The technique is based upon an extension of the integral-mode time-of-flight method $[158]$ for the low intensity regime, which accounts for capacitive effects associated with the sandwich-type thin-film device structure. In contrast to TDCF, the proposed method does not suffer from limitations induced by $R C$ effects, allows for a sensitive measurement of charge carrier density at very low pulse fluence without a reduced signal accuracy, and does not require ultrasensitive fast pre-amplifiers. The new method, however, has a more limited voltage range than TDCF. The analytical framework behind the technique, low intensity integral time-of-flight [LIITOF], is derived and verified by drift-diffusion [DD] simulations. The technique is applied to thin-film organic and perovskite solar cells and the field dependent external generation efficiency [EGE] is probed finding a good agreement of experimental results obtained via LIITOF and TDCF conducted on the same devices. 


\subsection{Experimental scheme of TDCF and LIITOF}

The schematic and circuit diagram of the LIITOF method is shown in Fig. 4.1 a,b. Here, a large load resistor and an external voltage source [to provide $V_{0}$ to the circuit] are connected in series with the device under test [DUT]. A short laser pulse is used to generate charge carriers in the bulk of the DUT. The voltage transients are recorded with an oscilloscope put in parallel to the DUT.

In particular, a diode-pumped, Q-switched Nd:YAG laser [Quantel, Viron Version A] operating a $532 \mathrm{~nm}$ excitation wavelength, $6.84 \mathrm{~ns}$ pulse width, and $20 \mathrm{~Hz}$ repetition rate is used in combination with a Standa 10MVAA attenuator to generate charge carriers in the bulk of the DUT. A Keithley 2450 is used to apply voltages on the DUT, which is in series with a $1 \mathrm{M} \Omega$ load resistor. The voltage transients are recorded with an oscilloscope [Rohde \& Schwarz, RTM 3004] with $1 \mathrm{M} \Omega$ input resistance put in parallel with the DUT. For dark $C$ - $V$ measurements, a E5061B ENA Network Analyzer with modulation frequency of $1 \mathrm{kHz}$ and a video bandwidth of $10 \mathrm{~Hz}$ is used, respectively. The voltage drop across the DUT is measured by a Keithley 2450 .

(a)

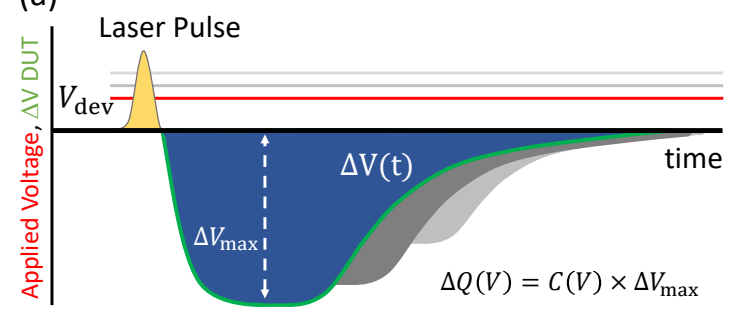

(b)

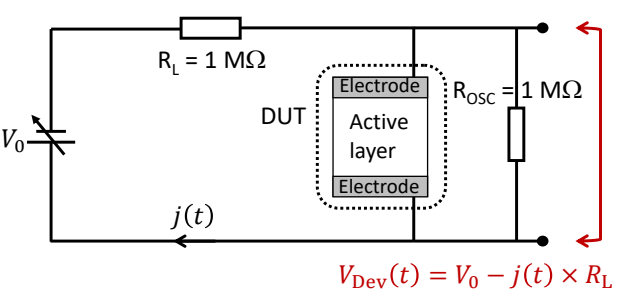

Figure 4.1: (a) Schematic timeline of a low-intensity integral time-of-flight [LIITOF] experiment. While the bias $V_{\mathrm{dev}}$ is applied on the device under test [DUT], a short laser pulse at $t=0$ photo-generates charge carriers in the DUT active layer. The photo-induced change in voltage drop across the DUT active layer is measured by an oscilloscope put in parallel with the DUT. The green [red] solid line indicates the corresponding photovoltage transient [applied device bias $V_{\mathrm{dev}}$ ]. (b) Circuit of a LIITOF experiment. A large load resistance $R_{\mathrm{L}}$ is in series circuit with the DUT, while the change in photo-induced voltage drop across the DUT is measured by an oscilloscope with large input resistance put in parallel to the DUT.

Fig. 4.2 a,b show a simplified schematic circuit and triggering diagram of a typical TDCF experimental setup. Here, a variable pre-bias $V_{\text {pre }}$ is applied on the operational photovoltaic device under test [DUT] using an external voltage source, while a short 
laser photo-pulse leads to the generation of charge carriers in the photoactive layer. After a certain delay time $t_{\text {delay }}$ the photogenerated charges are extracted by applying a collection bias $V_{\text {coll }}$ [typically a high reverse bias]. An oscilloscope is used to record the current flowing through the DUT, and by integrating the extraction photocurrent transient, the total number of extracted charge carriers can be obtained.

In particular, a Q-switched Nd:YAG laser [AOT1 picolo] with $0.162 \mu \mathrm{J} \mathrm{cm}^{-2}, \lambda_{\text {exc }}=532$ $\mathrm{nm}$, and $1 \mathrm{~ns}$ delay time is used to generate a short laser pulse exciting charge carriers in the bulk of the DUT. While an Agilent $81150 \mathrm{~A}$ pulse generator provides pre- and collection bias voltages, an oscilloscope from Agilent [DSO9104H] is used to record the current transients. Details of the TDCF setup are provided elsewhere, and are outside the scope of this thesis. 159

(a)

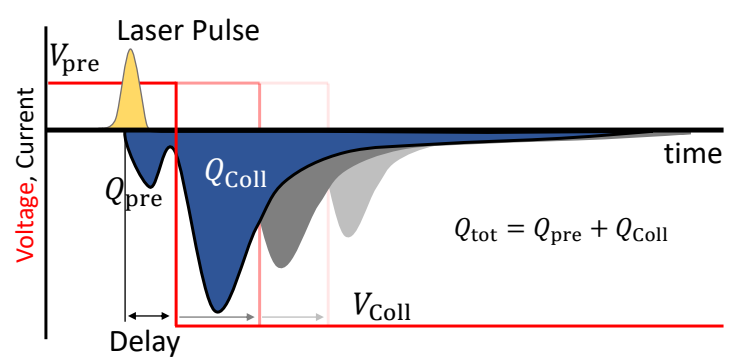

(b)

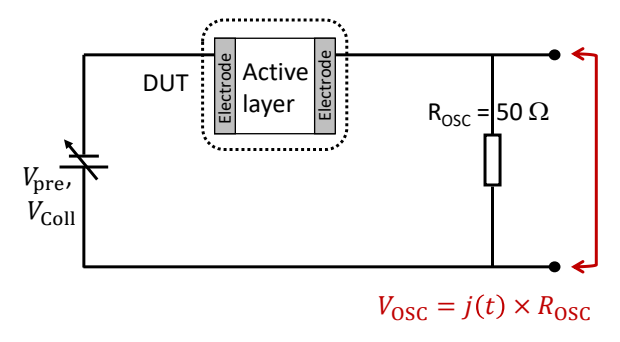

Figure 4.2: (a) Schematic timeline of a time delayed collection field [TDCF] experiment. At $t=0$, a short laser pulse photo-generates charge carriers in the active layer of a device under test [DUT], while it is hold under a pre-bias $V_{\text {pre }}$. After a short delay time, a high reverse collection bias $V_{\text {coll }}$ is applied on the DUT to extract all photogenerated charge carriers. The red [black] solid line indicates the corresponding applied voltage [photocurrent] transient. (b) Simplified circuit of a TDCF experiment, where the DUT is in series circuit with an oscilloscope with $R_{\mathrm{osc}}=50 \Omega$ input impedance. 


\section{LOW INTENSITY INTEGRAL TIME-OF-FLIGHT TECHNIQUE}

\subsection{Theoretical framework of LIITOF}

In LIITOF, the sandwich-type thin-film diode or solar cell device is connected in series with a large load resistance $R_{\mathrm{L}}$ and a voltage source applying a DC bias $V_{0}$. The device is initially kept under DC conditions, with the corresponding voltage drop across the device being given by $V_{\mathrm{dev}}=V_{0}-j_{0} R$, where $j_{0}$ is the [small] DC current density through the circuit and $R=R_{L} \times A$ is the load resistance times the device area $A$. At $t=0$, a light pulse is applied to the device, resulting in charge carriers being generated inside the active layer. The photogenerated electrons and holes are subsequently transported under the influence of the internal electric field towards the cathode and anode, respectively, giving rise to a photo-induced voltage transient across the device. In general, with the anode assumed to be located at $x=0$ and the cathode at $x=d$ [ $d$ denotes the active layer thickness], the corresponding total time-dependent current density [induced by the photogenerated charge carriers drifting to their respective electrodes] is independent of the position $x$ in the device and given by [79]:

$$
j_{t}=j_{c}(x, t)+\varepsilon \varepsilon_{0} \frac{\partial E(x, t)}{\partial t},
$$

where $E(x, t)$ is the electric field and $j_{c}(x, t)$ is the conduction current density given by the sum of the individual electron and hole current densities, which both, on the other hand, depend on the position $x$ in the active layer and the time $t ; \varepsilon$ is the relative permittivity, and $\varepsilon_{0}$ is the permittivity of the vacuum. The associated time-dependent voltage drop across the active layer is given by $V(t)=V_{0}-j(t) R$. Furthermore, the photo-induced change in the voltage drop $\Delta V(t)=V(t)-V_{\mathrm{dev}}$ is related to the change of the electric field within the active layer via:

$$
\frac{\partial \Delta V(t)}{\partial t}=\int_{0}^{d} \frac{\partial E(x, t)}{\partial t} d x
$$

Subsequently, upon taking the spatial average over the active layer of the total current in Eq. 4.1, and making use of Eq. 4.2, the following expression is obtained:

$$
\frac{\Delta V(t)}{R}+C_{\mathrm{geo}} \frac{\partial \Delta V(t)}{\partial t}=-\Delta \bar{j}_{c}(t)
$$

where $\Delta \bar{j}_{c}(t)=(1 / d) \int_{0}^{d} j_{c}(x, t) d x-j_{0}$ is the difference between the spatially averaged conduction current densities induced by the light pulse [note that $\Delta \bar{j}_{c}(t)=0$ for $t<0$ ], 
while $C_{\text {geo }}=\frac{\varepsilon \varepsilon_{0}}{d}$ is the geometrical capacitance of the active layer. For large load resistances $\left[R_{L} \rightarrow \infty\right.$, corresponding to $\left.t \ll R C_{\text {geo }}\right]$, Eq. 4.3 simplifies to $\partial \Delta V(t) / \partial t=$ $-\Delta \bar{j}_{c}(t) / C_{\text {geo }}$. Under these conditions, the maximal induced change in the voltage is given $\Delta V_{\max }=\Delta Q / C_{\text {geo }}$, where $\Delta Q=-\int_{0}^{t_{\text {extr }}} \Delta \bar{j}_{c}(t) d t$, is the total charge induced by the light pulse, while $t_{\text {extr }}$ is the time taken for all photogenerated charge carriers to be extracted at the electrodes. After accounting for non-uniform charge distributions in the active layer, it can be shown that $\Delta Q$ is related to the charge carrier densities inside the active layer via $160-162$

$$
\Delta Q=\frac{q}{d} \int_{0}^{d}[x \Delta p(x)+(d-x) \Delta n(x)] d x
$$

assuming negligible charge carrier recombination [i.e., low intensity condition] and no trapping during the extraction process $\left[0<t \leq t_{\mathrm{extr}}\right]$. Here, $\Delta p(x)=p(x, 0)-p\left(x, t_{\mathrm{extr}}\right)$ and $\Delta n(x)=n(x, 0)-n\left(x, t_{\text {extr }}\right)$, where $p(x, t)[n(x, t)]$ is the hole [electron] density within the active layer at position $x$ and time $t$.

In general, $\Delta p(x)$ and $\Delta n(x)$ can be expressed as $\Delta p(x)=n_{\mathrm{ph}}(x)+\Delta p_{0}(x)$ and $\Delta n(x)=n_{\mathrm{ph}}(x)+\Delta n_{0}(x)$, where $n_{\mathrm{ph}}(x)$ is the initial photogenerated carrier density at $t=0$ and $\Delta p_{0}(x)\left[\Delta n_{0}(x)\right]$ is the related induced change in the dark background hole [electron] density inside the active layer. In the case of an undoped device with non-injecting contacts, the background densities are negligibly small, and the active layer may be treated as an insulator; for this simplified case, Eq. 4.4 reduces to $\Delta Q=q n \bar{n}_{\mathrm{ph}} d$, where $\bar{n}_{\mathrm{ph}} \equiv(1 / d) \int_{0}^{d} n_{\mathrm{ph}} d x$ is the spatial average of the photogenerated carrier density at $t=0$. However, most OSCs employ ohmic contacts. In these devices, there exists a non-zero dark background density of electrons and holes, diffused from the contacts, accumulating near the anode and cathode contact, respectively. [162] These dark charge distributions near the contacts effectively reduces the thickness of the insulator-like region in the active layer, resulting in an increased device capacitance relative to $C_{\text {geo }}$.

Accounting for the presence of dark charge carriers, Eq. 4.4 can be then expressed as $\Delta Q=q \bar{n}_{\mathrm{ph}} d-\Delta Q_{0}$. Here, $\Delta Q_{0}=-\frac{q}{d} \int_{0}^{d}\left[x \Delta p_{0}(x)+(d-x) \Delta n_{0}(x)\right] d x$ represents the corresponding charge induced by the difference between the background charge density profiles between $t=0$ and $t=t_{\text {extr. }}$. However, since the background charge carrier profiles are determined by the prevailing applied voltage and electric field distribution, in 


\section{LOW INTENSITY INTEGRAL TIME-OF-FLIGHT TECHNIQUE}

contrast to the photogenerated charge $q \bar{n}_{\mathrm{ph}} d, \Delta Q_{0}$ is capacitive, associated with a redistribution of the background charge profiles induced by the voltage change $\Delta V_{\max }$ across the device. For small voltage perturbations $\Delta V_{\max } \ll V_{\text {dev }}, \Delta Q_{0}=\left(\partial Q_{0} / \partial V\right) \Delta V_{\max }$ is thus expected. Provided that $t_{\text {extr }} \ll R C$ [large $R_{\mathrm{L}}$ ],

$$
\bar{n}_{\mathrm{ph}}=\frac{C}{q d} \Delta V_{\max }
$$

is obtained, where

$$
C=C_{\text {geo }}+\frac{\partial Q_{0}}{\partial V}
$$

is the voltage-dependent [steady-state] capacitance of the device in the dark at $V=$ $V_{\mathrm{dev}}$. Hence, by measuring $\Delta V_{\max }$ via LIITOF as a function of the voltage $V_{\mathrm{dev}}$, in conjunction with device capacitance $C$, allows for $\bar{n}_{\mathrm{ph}}$ versus $V_{\mathrm{dev}}$ to be calculated, which [for a fixed light intensity] reflects the corresponding external generation efficiency [EGE]. 


\subsection{Limitations of LIITOF and how to address them}

To verify the analytical treatment, it was first applied to the result obtained from time-dependent drift diffusion [DD] simulations. The details of the DD model have been provided elsewhere 80, 81, and are outside the scope of this thesis. 162 Briefly, in the simulations, a trap-free and undoped active layer with a thickness of $100 \mathrm{~nm}$, a dielectric constant $\varepsilon=3$, balanced mobilities of $10^{-4} \mathrm{~cm}^{2} \mathrm{~V}^{-1} \mathrm{~s}^{-1}$, and a bimolecular recombination coefficient of $\beta=5 \times 10^{-12} \mathrm{~cm}^{3} \mathrm{~s}^{-1}$ were assumed. Furthermore, ohmic contacts that are perfectly selective for the extraction of electrons and holes at the cathode and anode contact, respectively, were assumed. The device was assumed to have an electrical device area of $A=0.04 \mathrm{~cm}^{2}$ and to be connected in series with a large load resistance of $R_{\mathrm{L}}=1 \mathrm{M} \Omega$, corresponding to $R=4 \times 10^{4} \Omega \mathrm{cm}^{2}$. With the geometric capacitance of the device given by $C_{\text {geo }}=26.56 \mathrm{nF} \mathrm{cm}^{-2}$, this amounts to $R C_{\text {geo }} \approx 1$ ms. Finally, the photogenerated carriers [introduced at $t=0$ ] were generated with a uniform rate inside the active layer, with the corresponding density $\bar{n}_{\mathrm{ph}}=n_{\mathrm{ph}}$ assumed to be directly proportional to the pulse fluence.

Fig. 4.3 a shows the simulated voltage transients [solid lines] for different $V_{\text {dev }}$ ranging between $-1 \mathrm{~V}$ and $0.7 \mathrm{~V}$. The corresponding $\Delta V_{\max }$ are plotted as a function of pulse fluence for different $V_{\text {dev }}$ in Fig. 4.3 b. In Fig. 4.3c, on the other hand, the device capacitance $C$ under steady-state conditions in the dark [corresponding to low frequencies] is simulated as a function of $V_{\mathrm{dev}}$. In general, it can be seen that $\Delta V_{\max }$ follows a linear dependence with the fluence at small $\Delta V_{\max }$. At large enough fluences, however, $\Delta V_{\max }$ eventually deviates from linearity as both higher order recombination and screening of the prevailing electric field start to play a role [as $\Delta V_{\max }$ becomes comparable to $\left.V_{\mathrm{dev}}\right]$. On the other hand, $\Delta V_{\max }$ is seen to strongly depend on $V_{\mathrm{dev}}$ at low fluences. It is worth noting that this dependence is present even for the idealized case when no recombination of charge carriers is present $[\beta=0$, dashed lines]. Instead, the $V_{\text {dev }}$ dependence of $\Delta V_{\max }$ is a consequence of the associated induced redistribution of the dark background charge carrier profile inside the active layer. As $V_{\mathrm{dev}}$ is increased, the diffusion of injected dark charges [from the electrodes] penetrates deeper into the bulk, effectively reducing the thickness of the neutral [insulator-like] region in active layer, manifested as an increased device capacitance relative to the geometrical capacitance $C_{\text {geo }}[$ see Eq. 4.6. 

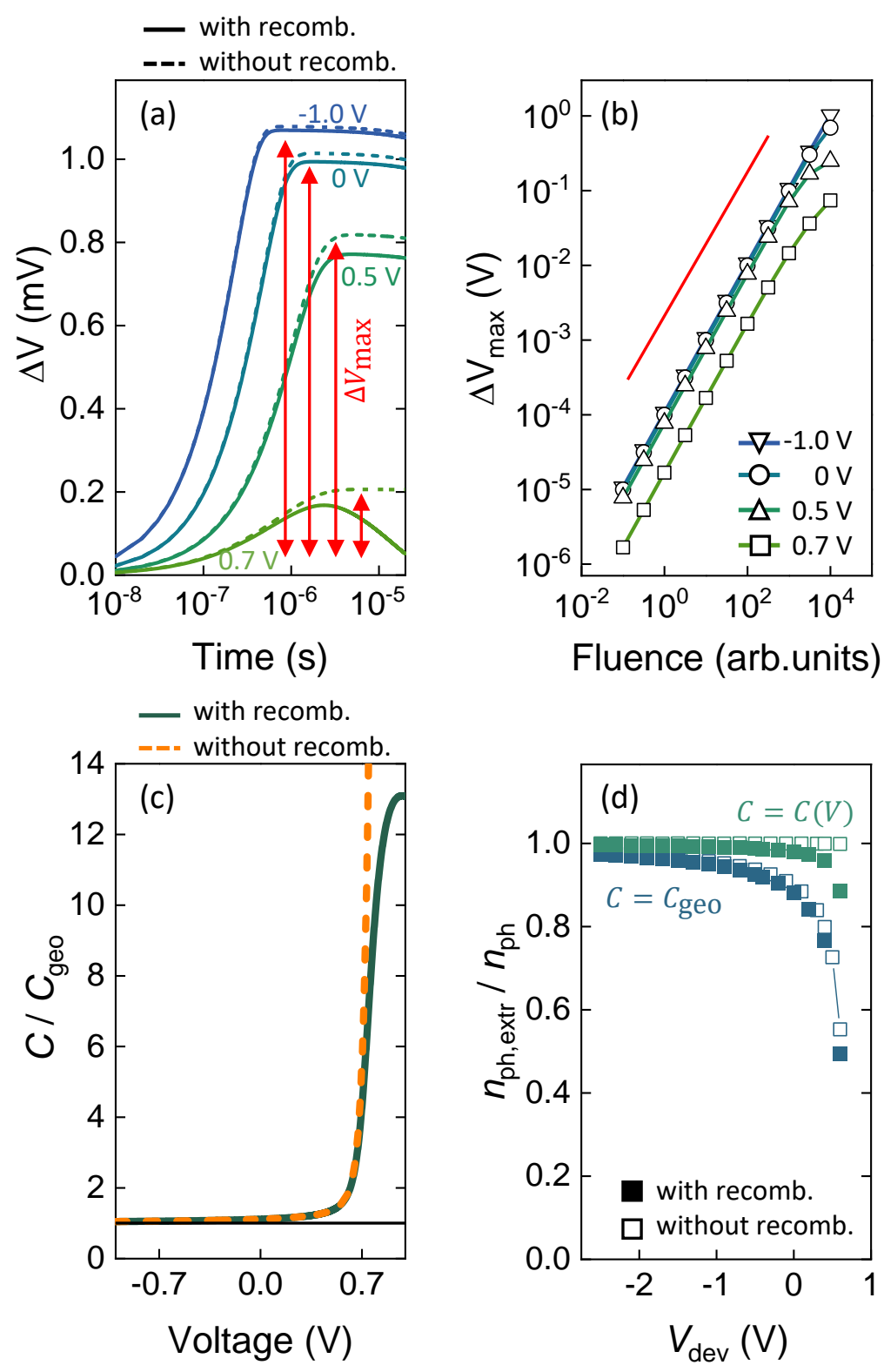

Figure 4.3: (a) Simulated voltage transients for different applied device voltages $V_{\text {dev }}$, and compared for the cases with [solid lines] and without [dashed lines] recombination. (b) Voltage transient maxima, $\Delta V_{\max }$, as obtained from the simulated voltage transients, plotted as a function of laser pulse fluence. The red solid line is a guide to the eye with a slope of 1. (c) Simulated device capacitance plotted as a function of applied voltage. The capacitance is normalized to the geometrical device capacitance $C_{\text {geo }}$ [vertical black line]. The case with [without] recombination is indicated by solid [dashed] lines. (d) The extracted charge carrier density $\left[n_{\mathrm{ph}, \text { extr }}\right]$, normalized to the generated carrier density $\left[n_{\mathrm{ph}}\right]$, as obtained from the simulated voltage transients, and plotted as a function of device voltage $V_{\mathrm{dev}}$. Filled [open] symbols correspond to the case with [without] recombination. 
Fig. 4.3d shows the extracted charge carrier density $n_{\text {ph,extr }}$, as obtained from the simulations using Eq. 4.5, relative to the input photogenerated carrier density $n_{\mathrm{ph}}$. Indeed, $n_{\mathrm{ph} \text {,extr }}$ is closely given by $n_{\mathrm{ph}}$ when the device capacitance $C(V)$ [Fig. 4.3c] is used in Eq. 4.5. In contrast, if $C=C_{\text {geo }}$ is assumed instead, a deviation between $n_{\mathrm{ph}, \mathrm{extr}}$ and $n_{\mathrm{ph}}$ is observed, which may be mistaken as an apparent field dependence of EGE; hence, to correctly obtain $n_{\mathrm{ph}}$, the voltage dependence of the device capacitance must be accounted for. Is it worth noting that there is a small deviation taking place between the cases with [fully filled symbols] and without [open symbols] recombination in the active layer at large $V_{\text {dev }}$ approaching the built-in voltage; this deviation can be attributed to additional [pseudo-] first-order recombination taking place between photogenerated charge carriers and dark background charge carriers [independent of light intensity] near the electrodes. 152, 163. In principle, this additional loss may be minimized by tuning the optical electric field [e.g., careful choice of the laser wavelength or the introduction of optical spacer layer] such that the generation profile peaks in the middle of the active layer and is minimal near the electrodes. It should be stressed that in case of non-ideal contacts, surface recombination [i.e., the collection of minority carriers at the 'wrong' electrode] may become prevalent, presenting an additional voltage dependent first-order recombination channel. [164]

From the above presented theoretical and numerical analysis it can be concluded that [photogenerated] charge carrier densities in thin-film solar cells can be measured sensitively via LIITOF, when (i) higher-order recombination processes are not present, and (ii) [voltage dependent] carrier back-injection and diffusion-mediated redistribution of dark background charges in the photoactive layer of the DUT are accounted for. While (i) can be addressed by recording LIITOF voltage transients at low pulse fluence and avoiding too high $\Delta V_{\max }\left[\Delta V_{\max }\right.$ should be as small as possible, preferably well below $10 \mathrm{mV}$ ], (ii) can be addressed by accurately measuring the voltage dependent device capacitance in dark. In the following Section 4.5, these findings will be implemented and used to probe the EGE on different thin-film organic and perovskite solar cell devices. Details of the device fabrication are provided in Appendix A. 


\subsection{Field dependence of external generation efficiency}

Fig. 4.4 a shows the dark capacitances of all three devices [i.e., neat PCDTBT, PCDTBT:PC ${ }_{70} \mathrm{BM}$ (1:4), and triple cation perovskite] plotted as a function of device voltage, $V_{\mathrm{dev}}$. As shown, the $\mathrm{PCDTBT}: \mathrm{PC}_{70} \mathrm{BM}(1: 4)$ and perovskite thin-film solar cell show changes in device capacitance when $V_{\mathrm{dev}}$ is approaching $V_{\mathrm{bi}}$. Great care was taken to avoid too high and bimolecular recombination-inducing laser pulse fluences when recording the voltage transients at different $V_{\text {dev }}$. Fig. 4.4 $\mathrm{b}$ shows the $\Delta V_{\max }$ at short-circuit conditions, as obtained from the voltage transients, plotted as a function of laser pulse fluence, and compared for all three thin-film solar cell devices. The red solid line in Fig. 4.4 $\mathrm{b}$ is a guide to the eye with a slope of 1 indicating the absence of higher-order recombination processes. The relations between the applied circuit voltages $V_{\text {appl }}$ and the measured voltage drops $V_{\text {dev }}$ across the three thin-film devices are depicted in Fig. 4.4c. The LIITOF voltage transients are shown in Fig. 4.4d-f, from which $\Delta V_{\max }$ were obtained at the corresponding voltage plateaus.

From the $C$ - $V$ curves and voltage transients, the EGEs were calculated, which are determined based upon the photogenerated charge carrier density $\left[n_{\mathrm{ph}}\right]$ and the pulse photon density $\left[N_{\mathrm{ph}}\right]$ via $\mathrm{EGE}=\frac{n_{\mathrm{ph}}}{N_{\mathrm{ph}}}$, where $N_{\mathrm{ph}}=\frac{\lambda F}{h c A d}, \lambda$ is the laser pulse excitation wavelength, $h$ the Planck constant, and $F$ denotes the pulse fluence [in the unit of $J$. These LIITOF results were cross-correlated with those obtained from TDCF conducted on the same devices. Fig. 4.5a compares the current density versus applied voltage $[J-V]$ curve [details to $J-V$ measurements are provided in Box 3.1] of the PCDTBT:PC ${ }_{70} \mathrm{BM}$ (1:4) solar cell [solid line] with the EGE obtained via LIITOF [red symbols] and TDCF [orange symbols]. The EGE in PCDTBT:PC 70 BM (1:4) shows a weak field dependence decreasing slightly at increasing forward bias voltages. Thus the LIITOF EGE results are in excellent agreement with those obtained via TDCF. It should be noted, however, that due to uncertainties in the device capacitance measured at high voltages [i.e., when $V_{\text {dev }}$ approached $V_{\text {bi }}$, the trustable $V_{\text {dev }}$ regime in LIITOF was limited to $\sim 0.47 \mathrm{~V}$ in forward bias direction. This was partly due to the rapid increase of the capacitance with voltage [see Fig. 4.4 a], where the value of $C$ becoming more sensitive to small voltage fluctuations $\left[\Delta V_{\max }\right]$, and partly due to strong recombination and space charge effects affecting the measured capacitance at large bias. 

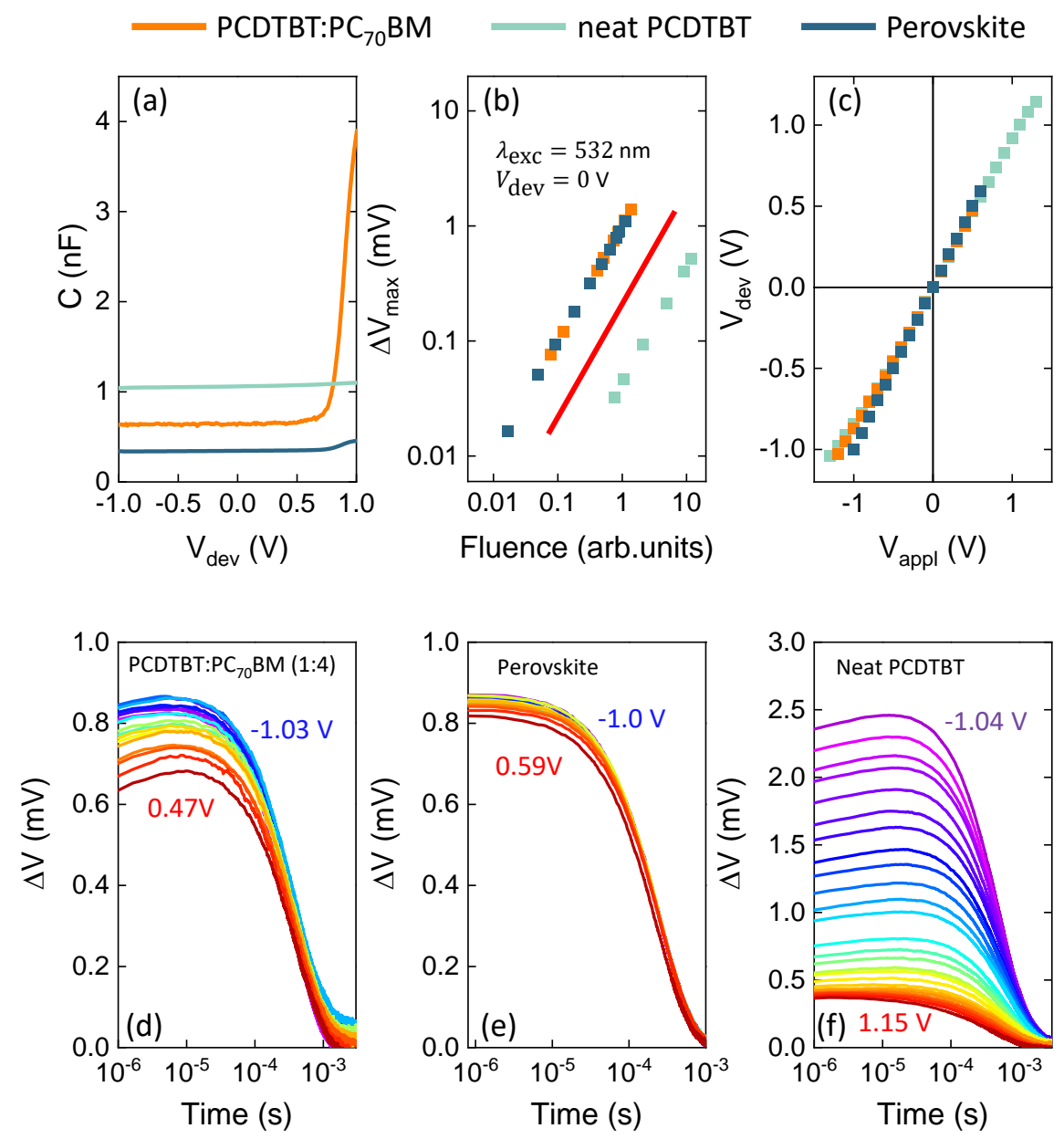

Figure 4.4: (a) Device capacitance in dark plotted as a function of voltage, and compared for a PCDTBT:PC ${ }_{70} \mathrm{BM}(1: 4)$, neat PCDTBT and perovskite thin-film solar cell. A video bandwidth of $10 \mathrm{~Hz}$ and modulation frequencies of $1 \mathrm{kHz}$ [PCDTBT:PC ${ }_{70} \mathrm{BM}$ (1:4), neat PCDTBT] and $5 \mathrm{MHz}$ [perovskite] was used. (b) Maximum change $\Delta V_{\max }$, as obtained from voltage transients, for all three solar cells plotted as a function of laser pulse fluence. The excitation wavelength was set to $\lambda_{\text {exc }}=532 \mathrm{~nm}$, and no bias voltage was applied on the devices [short-circuit]. The red solid line is a guide to the eye with a slope of 1 indicating the absence of higher-order photocurrent loss mechanisms. (c) Relation between applied circuit voltages and the measured voltage drops across the three devices. (d) Voltage transients of a PCDTBT:PC ${ }_{70} \mathrm{BM}$ (1:4) thin-film solar cell compared for different applied bias voltages. (e) Repetition of panel (d), but plotted for a neat PCDTBT solar cell. (f) Repetition of panel (d), but plotted for a perovskite thin-film solar cell.

The EGE in a thin-film perovskite solar cell [see Fig. 4.5b] was found again to be field-independent. The LIITOF results [red symbols] showed good agreement with those obtained via TDCF. Similar to the PCDTBT:PC 70 BM (1:4) device, the trustable $V_{\mathrm{dev}}$ 


\section{LOW INTENSITY INTEGRAL TIME-OF-FLIGHT TECHNIQUE}

window was, when probed by LIITOF, limited to $\sim 0.6 \mathrm{~V}$ in forward bias direction. Finally, a system with electric field dependent EGE was investigated - to this end, a neat PCDTBT thin-film device was used. It is well-established that single-component organic solar cells exhibit weak and strongly field dependent charge generation. [46, 163] Therefore, neat PCDTBT device was an appropriate model system to observe the field dependence. It is worth noting that the capacitance of this device showed a weaker voltage dependence [see Fig. 4.4 a], allowing for the capacitance to be accurately measured over the entire voltage range. Subsequently, as shown in Fig. 4.5c, the field-dependent EGE results obtained via LIITOF [red symbols] and TDCF [orange symbols] were in excellent agreement over the entire bias voltage regime.

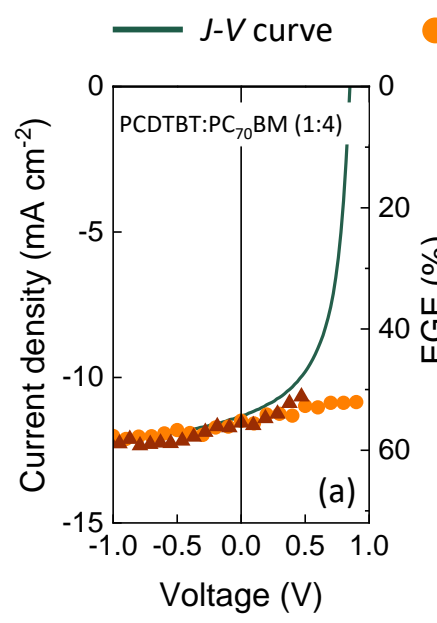

\section{EGE obtained via TDCF}

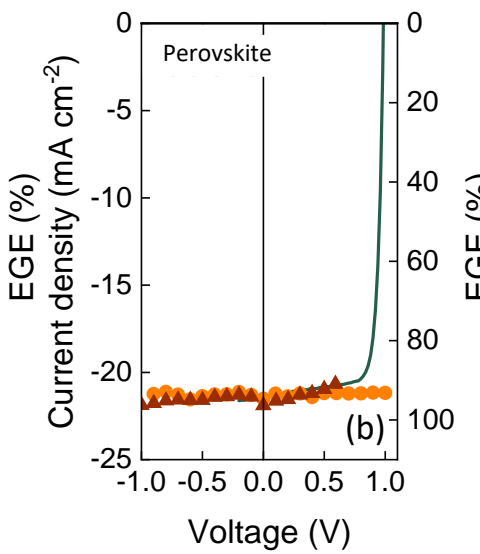

AGE obtained via LIITOF

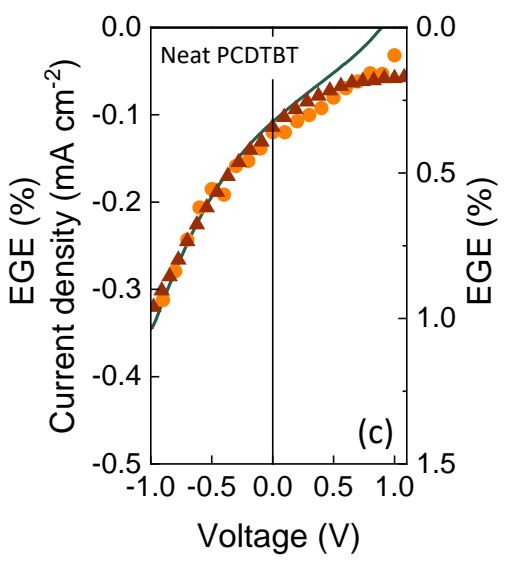

Figure 4.5: (a) Current density versus device voltage $[J-V]$ performance [solid line] of a thin-film PCDTBT:PC $\mathrm{P}_{70} \mathrm{BM}$ (1:4) solar cell measured under artificial 1 sun [AM $1.5 \mathrm{G}$ condition] illumination, and compared with the external generation efficiency [EGE] obtained via TDCF [orange symbols] and LIITOF [red symbols]. (b) Repetition of panel (a) but plotted for a thin-film perovskite solar cell. (c) Repetition of panel (a) but plotted for a neat PCDTBT device.

In contrast to the PCDTBT:PC ${ }_{70} \mathrm{BM}(1: 4)$ and perovskite thin-film device, the accuracy of the neat PCDTBT $C$ - $V$ measurement was not limited at large forward bias voltages by carrier diffusion and back-injection from the electrodes into the photoactive layer - this can mainly be attributed to the non-ohmic injection character of one electrode of this device stack suppressing strong recombination and space charge effects at large voltages. In this regard, it should be noted that the EGE is a property of the 
photoactive layer, hence a modification of the device stack aiming towards a precise $C$ - $V$ measurement [or, suppression of diffusion of injected dark charges, recombination, and the build-up of space charge] allows for accurate LIITOF measurements over the entire voltage regime [i.e., even for $V_{\mathrm{dev}} \geq V_{\mathrm{bi}}$ ]. 


\section{LOW INTENSITY INTEGRAL TIME-OF-FLIGHT TECHNIQUE}

\subsection{Conclusion}

In this chapter, a transient measurement technique, that is, low intensity integral timeof-flight, was described, which is based upon the sensitive measurement of photo-pulse induced changes in voltage drop across the active layer, combined with capacitance measurements. A simple series-circuit with large $R C$-time was used to generate voltage transients at low laser pulse fluence from which the maximum change in active layer voltage drop could be determined. The theoretical framework of LIITOF was derived and verified by DD simulations, and its applicability was demonstrated by probing the field dependence of EGE in thin-film perovskite and organic solar cells. The results were in good agreement with experimental results obtained via TDCF conducted on the same devices.

Despite the limitations of LIITOF at high forward bias voltages due to uncertainties in the accurate measurement of the device capacitance, LIITOF is operating at very low pulse fluence [hence, avoiding any parasitic higher-order recombination] and does not suffer from $R C$-time limitations. Subsequently, LIITOF allows with its much simpler circuit to measure small charge carrier densities sensitively, and can be used complementary to the rather complex TDCF to probe the field dependence of charge generation in thin-film solar cells. 


\section{Chapter 5}

\section{Light intensity dependence of photocurrent}

The competition between recombination and extraction of carriers defines the charge collection efficiency and thereby the overall performance of photovoltaic devices. This competition is particularly important in light-harvesting devices made from disordered and/or low permittivity materials such as organic semiconductors. Time resolved electrical and optical techniques are often employed to investigate different loss mechanisms in such systems. In this chapter, the steady-state light intensity dependence of the photocurrent [so-called IPC] and charge collection efficiency under operational conditions is clarified, and shown how different loss mechanisms can be identified based upon their unique signatures. In particular, it is shown how IPC can be used to distinguish firstorder, trap-assisted recombination from other first-order photocurrent loss mechanisms. The theoretical framework is advanced and verified by a one-dimensional drift-diffusion device model which further enhances the analysis. Finally, the extended IPC analysis is demonstrated on organic thin-film solar cell devices. Thus, the relatively straightforward measurement of light intensity dependent photocurrent over a large dynamic range can be a powerful tool for understanding photovoltaic device fundamentals and optimising performance.

This chapter is written based upon a collaborative work by the author submitted to the journal Physical Review Applied in 2020. 


\section{LIGHT INTENSITY DEPENDENCE OF PHOTOCURRENT}

\subsection{Photocurrent generation and collection efficiency}

The performance of organic solar cells is ultimately dictated by the competition between the recombination and extraction of photo-generated charge carriers. [165] As described in detail in Chapter 1, Section 1.5, the working principle of excitonic, bulk-heterojunction [BHJ] devices involves the formation of singlet excitons in either the electron donor [D: p-type] or acceptor [A: n-type], a D-A interfacial charge transfer $[\mathrm{CT}]$ state, and a charge separated state after CT dissociation into free and mobile charge carriers. 27, 46, 166. Given that not only excitons and CT states can recombine back to the ground state [GS], but also free charge carriers may encounter each other and recombine via non-geminate recombination, it becomes clear that recombination is the main loss channel limiting the charge collection efficiency $\left[\eta_{\text {col }}\right]$ in BHJ solar cells, ultimately limiting the short-circuit current density $\left[J_{\mathrm{sc}}\right]$ and the power conversion efficiency [PCE] as well. [166], 167]

The charge collection efficiency describes the probability for charge carriers, once generated in the active layer, to be extracted at the contacts and avoid recombination. For a constant carrier recombination lifetime $\tau$, the charge collection efficiency generally takes the form

$$
\eta_{\mathrm{col}}=\frac{\tau}{t_{\mathrm{tr}}} \times\left[1-\exp \left\{-\frac{t_{\mathrm{tr}}}{\tau}\right\}\right]
$$

in accordance with the Hecht equation, [167] where $t_{\text {tr }}$ denotes the drift-transport time of charge carriers. Under these conditions, the recombination kinetics are first-order and the short-circuit current is expected to vary linearly with light intensity $I_{\mathrm{L}}$; thus following a $J_{\mathrm{sc}} \propto I_{\mathrm{L}}^{\alpha}$ dependence with $\alpha=1$. Here the parameter $\alpha$ is introduced defining the slope of $J_{\mathrm{sc}}$ versus $I_{\mathrm{L}}$ in a $\log$-log plot with $\alpha=d \log \left(J_{\mathrm{sc}}\right) / d \log \left(I_{\mathrm{L}}\right)$.

This type of first-order kinetics is commonly associated with trap-assisted recombination of charge carriers taking place via deep trap states in the gap, with the associated recombination rate scaling linearly with the carrier density [see Chapter 1, Section 1.8.2. In organic solar cells composed of low mobility semiconductors, however, at operational light intensities the carrier diffusion length is often of similar order to the Coulomb capture radius, resulting in non-geminate, bimolecular recombination [see Chapter 1, Section 1.8.1 dominating. 


\subsubsection{Techniques to probe the photocurrent loss mechanisms}

The fact that different recombination mechanisms scale differently with the generation rate and carrier density has been utilized to understand recombination processes in solar cells by using light intensity dependent open-circuit voltage $\left[V_{\mathrm{oc}}\right]$ and photocurrent $[\mathrm{IPC}]$ measurements as well as transient photovoltage $[\mathrm{TPC}]$ and charge extraction [CE] techniques.

In TPV, the recombination dynamics are probed by measuring the excess charge carrier lifetime by maintaining the device under steady-state illumination at open-circuit and applying a short light pulse. 167 In CE, on the other hand, the current transient is measured after the device is switched from open-circuit [under steady-state illumination] to short-circuit [with no illumination]. 167] By varying the steady-state illumination and open-circuit voltage, TPV and CE allow for the determination of the recombination rate constant and its kinetics. However, it has been recently shown that both techniques suffer from parasitic, capacitive effects limiting the trustworthy measurement range. [162, 168] In particular, at low light intensities, corresponding to low open-circuit voltages, device capacitive effects, $R C$-time constants and non-uniform charge carrier distributions can lead to a misjudgement of the recombination order.

Light intensity dependent $V_{\text {oc }}$ measurements, on the other hand, rely on the determination of the [light] ideality factor $n_{\text {id }}$ of the solar cell used to estimate the predominant recombination process. Here, [light] ideality factors of $n_{\text {id }}=1$ [bimolecular free-carrier recombination], $n_{\mathrm{id}}=2$ [expected for first-order trap-assisted recombination via midgap states] or $1<n_{\text {id }}<2$ [often attributed to trap-assisted recombination via exponential tail states] are usually observed.[47, 48, 169] However, these measurements are very sensitive to parasitic leakage currents at low light intensities and surface recombination of charge carries at the electrodes at high light intensities, which can considerably narrow the applicable intensity range at which $n_{\text {id }}$ is determined. [47, 81] This is quite often not appreciated.

Intensity dependent photocurrent [IPC] measurements offer the possibility to investigate the photocurrent losses as a function of the incident light intensity under different operational conditions and to identify loss mechanisms [e.g., bimolecular recombination], which become dominant at particular light intensities. 129, 154, 170-172. The IPC for organic solar cells has been also extensively used to study the role of charge 


\section{LIGHT INTENSITY DEPENDENCE OF PHOTOCURRENT}

carrier mobility [173], recombination rate constant [129] and space charge effects[154]. However, since incomplete dissociation of excitons and CT states is also first-order, it has been difficult to distinguish first-order, trap-assisted recombination from the former based upon IPC measurements. Additionally, it has also been shown that non-geminate recombination between photo-generated and equilibrium majority charge carriers near the contacts exhibits pseudo-first order dynamics 152.

Given this background, it is evident that methods to reliably quantify and distinguish different recombination mechanisms, especially those that are of first-order, are currently lacking. In this chapter, the influence of different first-and-higher order photocurrent loss mechanisms on the device photocurrent and charge collection efficiency is clarified and supported by drift-diffusion simulations. In particular, it is demonstrated how IPC, when performed sensitively over a broad range of light intensities, can be used to unambiguously differentiate between first-order trap-assisted recombination from other first-order losses. This sets IPC apart from other techniques commonly used to study recombination losses in organic solar cells and opens a new window to investigate the absolute photocurrent loss induced by first-order, trap-assisted recombination. The presented results are very general and also apply to other photovoltaic systems including indoor photovoltaic applications, concentrator solar cells and photodetectors. 


\subsubsection{Basic considerations for charge collection efficiency}

The photocurrent [density] at a given excitation wavelength $\lambda$ and light intensity $I_{\mathrm{L}}$ is directly related to the photovoltaic external quantum efficiency [EQE]. At short-circuit, $J_{\mathrm{ph}}=J_{\mathrm{sc}}$, the corresponding EQE is thus

$$
\mathrm{EQE}=\frac{h c}{\lambda q} \times \frac{J_{\mathrm{sc}}}{I_{\mathrm{L}}}
$$

where $h$ is the Planck constant, $c$ the speed of light in vacuum, and $q$ the elementary charge. Accordingly, for a linear photocurrent [density] versus light intensity, the corresponding EQE is expected to be constant. When higher-order photocurrent loss mechanisms are present, however, the charge collection efficiency $\eta_{\text {col }}$ becomes dependent on the carrier generation rate, which is reflected by a light intensity dependence of the EQE; in general, this may be expressed as

$$
\mathrm{EQE} \propto \eta_{\mathrm{col}} \propto I_{\mathrm{L}}^{S}
$$

where the parameter $S=d \log (\mathrm{EQE}) / d \log \left(I_{\mathrm{L}}\right)=\alpha-1$ is introduced defining the slope of EQE versus $I_{\mathrm{L}}$ in a log-log plot.

To clarify the effect of recombination on the intensity dependence of the charge collection efficiency, a one-dimensional drift-diffusion model has been used, [80, 81 treating the BHJ active layer as an effective semiconductor [although in reality it is a molecular blend of electron acceptor and donor]. For a given [average] generation rate $G$ of free [separated] charge carriers, the device model calculates the total current density based on the prevailing charge transport properties, space charge effects and recombination within the effective semiconductor layer. The charge collection efficiency at short-circuit is defined as

$$
\eta_{\mathrm{col}}=\frac{\left|J_{\mathrm{sc}}\right|}{q G d}
$$

where $d$ is the thickness of the active layer, and $G$ the photo-generation rate. For the simulations, a device having an active layer thickness of $100 \mathrm{~nm}$, with a permittivity of $\varepsilon=3.5$ and an electrical energy level gap of $1.3 \mathrm{eV}$, is assumed. Furthermore, the contacts are selective for the extraction of majority carriers, having injection barriers of $0.1 \mathrm{eV}$. The carrier photo-generation rate, assumed to be uniform throughout the 


\section{LIGHT INTENSITY DEPENDENCE OF PHOTOCURRENT}

active layer, depends linearly on the incident light intensity, i.e., $G \propto I_{\mathrm{L}}$. A light intensity of 1 sun is assumed to correspond to a photo-generation rate $G=1.1 \times 10^{22}$ $\mathrm{cm}^{-3} \mathrm{~s}^{-1}$. Unless otherwise stated, the active layer is assumed trap-free with balanced charge carrier mobilities $\mu_{\mathrm{n}}=\mu_{\mathrm{p}}$, where $\mu_{\mathrm{n}}\left[\mu_{\mathrm{p}}\right]$ is the electron [hole] mobility.

\subsection{Negligible recombination under ideal conditions}

Under ideal conditions when the recombination of photo-generated charge carriers is negligible, the magnitude of the photocurrent density at an applied device voltage of $V=0 \mathrm{~V}$ [short-circuit] is given by $\left|J_{\mathrm{ph}}\right|=q G d$. In this case the photocurrent is linear with respect to the light intensity [i.e., $J_{\mathrm{ph}} \propto G^{\alpha}$ with $\alpha=1$ ]. Under these ideal conditions, the charge collection efficiency is $100 \%$ and only first-order losses related to absorption and the charge carrier generation efficiency [geminate recombination of excitons or interfacial charge transfer states] are present. In real devices, however, there are always charge collection losses induced by recombination. In general, these losses can be the result of poor charge transport, increased recombination rates and accumulation of carrier densities inside the active layer, external resistive effects limiting the device current, or the presence of regions with low electric field caused by the buildup of space charge. 


\subsection{Bimolecular recombination and its impact on pho- tocurrent}

Here, the case with balanced charge transport with different degrees of second-order recombination in the active layer is considered. Fig. 5.1 a shows the simulated [solid and dashed line] photocurrent density as a function of light intensity, for different recombination constants $\beta=\zeta \beta_{\mathrm{L}}$. Here, $\zeta$ is the bimolecular recombination reduction factor relative to the Langevin rate $\beta_{\mathrm{L}}=q\left(\mu_{\mathrm{n}}+\mu_{\mathrm{p}}\right) /\left(\varepsilon \varepsilon_{0}\right)\left[\varepsilon_{0}\right.$ is the vacuum permittivity] [see Chapter 1, Section 1.8.1. The corresponding charge collection efficiency versus intensity is depicted in Fig. 5.1 b. The dashed black lines in Fig. 5.1 a,b, representing the ideal, trap-free case, are guides to the eye with slope parameters of $\alpha=1$ and $S=0$, respectively. As shown in Fig. 5.1 a [Fig. 5.1 b] when bimolecular recombination starts to dominate over charge extraction the photocurrent density [charge collection efficiency] versus intensity starts to deviate from linearity following a power of $1 / 2[-1 / 2]$ dependence. The onset point of the deviation depends on the bimolecular reduction factor and shifts to higher intensity with decreasing $\zeta$.
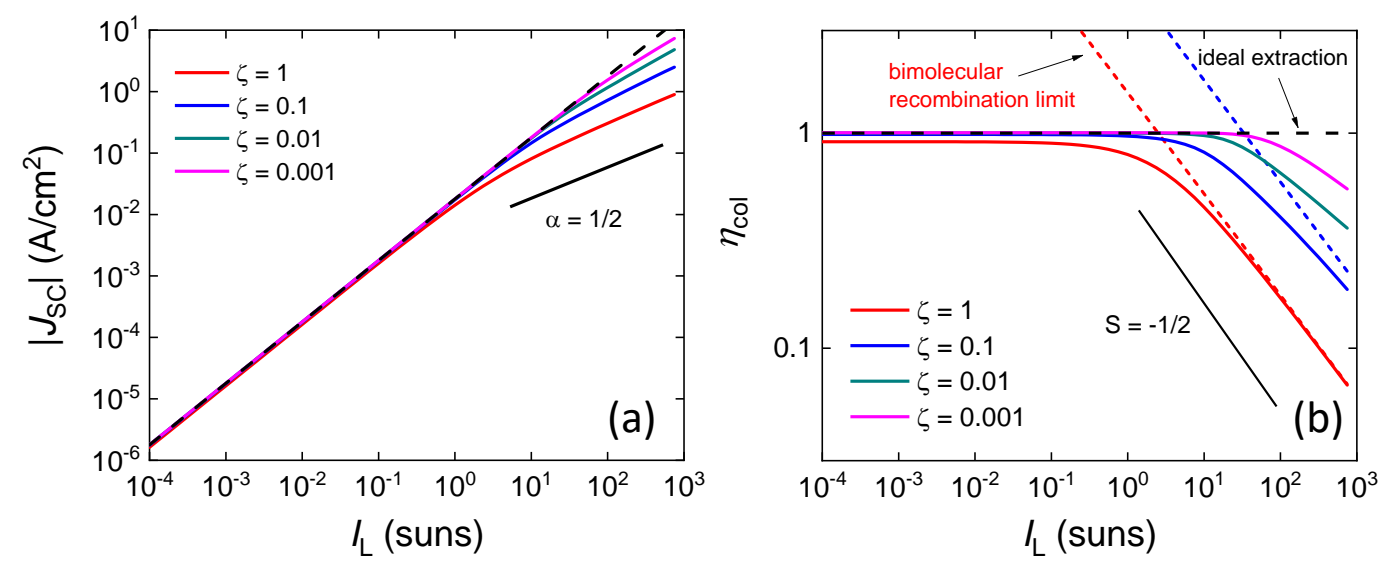

Figure 5.1: (a) Simulated short-circuit current density $\left[J_{\mathrm{sc}}\right]$ plotted as a function of light intensity $\left[I_{\mathrm{L}}\right]$, and compared for different bimolecular recombination reduction factors between $\zeta=0.001$ and $\zeta=1$. The black dashed line represents the case for ideal, lossfree charge extraction. (b) Repetition of panel (a), but instead the charge collection efficiency $\left[\eta_{\text {col }}\right]$ is plotted against the light intensity. The analytical expressions of Eq. 5.6 [coloured dotted lines] have been included assuming balanced mobilities of $\mu_{\mathrm{n}}=\mu_{\mathrm{p}}=10^{-4}$ $\mathrm{cm}^{2} \mathrm{~V}^{-1} \mathrm{~s}^{-1}$.

At high enough photo-generation rates, when the bimolecular recombination dominates over the charge extraction, the photocurrent becomes transport-limited. [139] Under 


\section{LIGHT INTENSITY DEPENDENCE OF PHOTOCURRENT}

these conditions, assuming a constant driving force $\left(d E_{\mathrm{F}, \mathrm{n}}\right) / d x=\left(d E_{\mathrm{F}, \mathrm{p}}\right) / d x \approx-q V_{\mathrm{oc}} / d$ for charge carriers $\left[E_{\mathrm{F}, \mathrm{n}}\left(E_{\mathrm{F}, \mathrm{p}}\right)\right.$ is the quasi-Fermi level for electrons (holes)], the upper limit for the short-circuit current set by second-order recombination is obtained from

$$
J_{\mathrm{ph}}=2 q \sqrt{\mu_{\mathrm{n}} \mu_{\mathrm{p}}} \times \sqrt{\frac{G}{\beta}} \times \frac{\left[V-V_{\mathrm{oc}}\right]}{d} .
$$

The associated charge collection efficiency at short-circuit in this limit is given by

$$
\eta_{\mathrm{col}}=\frac{L_{\mathrm{eff}}}{d}=\frac{2 \tau_{\beta} V_{\mathrm{oc}} \sqrt{\mu_{\mathrm{n}} \mu_{\mathrm{p}}}}{d^{2}}
$$

which depends on the carrier generation rate via the effective bimolecular recombination lifetime $\tau_{\beta}=1 / \sqrt{\beta G}$. As shown in Fig. 5.1 b, the simulations [solid lines] are in good agreement with the analytical limit at high photo-generation rates [coloured dotted lines], when bimolecular recombination dominates $L_{\text {eff }} / d \ll 1$. Therefore, in accordance with Eq. 5.6, a $J_{\text {sc }} \propto G^{\alpha}\left[\eta_{\text {col }} \propto G^{S}\right]$ with $\alpha=1 / 2[S=-1 / 2]$ at high light intensities is expected.

At low light intensities when $L_{\text {eff }} / d \gg 1$, in turn, second-order recombination is negligible, and it is expected that $J_{\mathrm{sc}} \propto G$. Under these conditions the collection efficiency is constant and close to unity, as most of the photo-generated carriers are extracted. It should be noted that the small deviations from $\eta_{\text {col }}=1$ at low intensities are caused by recombination between injected background carriers near the contacts and photogenerated carriers, manifest as pseudo-first order losses. [152] Another source of firstorder recombination loss is surface recombination, i.e., the extraction of the wrong carrier type, at the contacts. 164] These first-order losses are generally small at shortcircuit but may become important for thin active layers and at high injection/voltage levels. The parameter $L_{\text {eff }} / d$ determines the onset of the transition from the linear $[\alpha=1]$ to the nonlinear $[\alpha=1 / 2]$ photocurrent versus intensity regime where secondorder recombination dominates. The associated onset generation rate, corresponding to $L_{\mathrm{eff}} / d \approx 1$, can be approximated as $G^{*}=\frac{4 \varepsilon \varepsilon_{0} V_{\mathrm{oc}}^{2}}{q d^{4}} \times \frac{\mu_{\mathrm{n}} \mu_{\mathrm{p}}}{\left|\mu_{\mathrm{n}}+\mu_{\mathrm{p}}\right| \zeta}$ in terms of $\beta=\zeta \beta_{\mathrm{L}}$. Hence, the onset of second-order recombination predominantly depends on the ratio between the slower carrier mobility $\mu_{\mathrm{s}}$ and the [Langevin] reduction factor $\zeta$.129. 


\subsection{The effect of an external series resistance}

At high incident light intensities, the series resistance of the external circuit will eventually limit the photocurrent that can be extracted. For an externally applied voltage $V$, the actual applied voltage drop across the device is given by $V_{\mathrm{dev}}=V-J R_{\mathrm{S}}$, where $J R_{\mathrm{S}}$ represents the parasitic voltage drop across the rest of the circuit [i.e., electrodes, external wires, etc.] having a total series resistance of $R_{\mathrm{S}}$ in units of $\Omega \mathrm{cm}^{2}$. Subsequently, for $V_{\mathrm{ext}}=0$, the voltage drop across the device is given by $V_{\mathrm{dev}}=-J_{\mathrm{sc}} R_{\mathrm{S}}$. Therefore, at very high intensities [i.e., large currents], a significant voltage drop across the device, is expected.
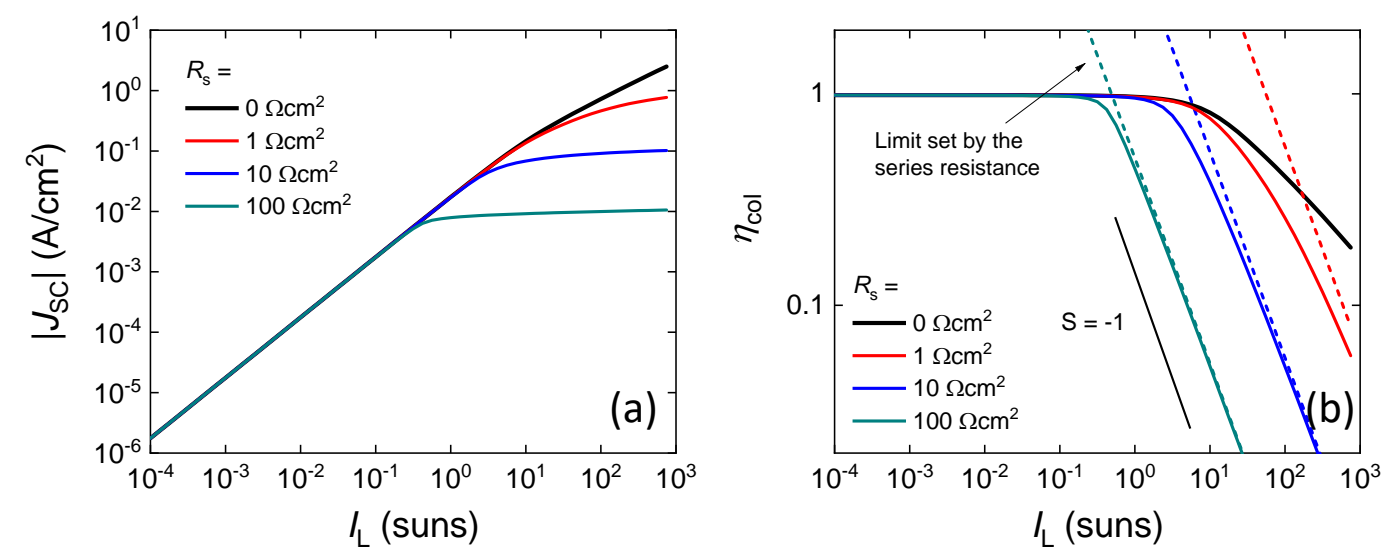

Figure 5.2: (a) Simulated short-circuit current density $\left[J_{\mathrm{sc}}\right]$ plotted as a function of light intensity, $\left[I_{\mathrm{L}}\right]$, and compared for different series resistances $R_{\mathrm{S}}$. At high light intensity $J_{\mathrm{sc}}$ tails off to the limit of $V_{\mathrm{oc}} / R_{\mathrm{S}}$ set by the series resistance $R_{\mathrm{S}}$. (b) Repetition of panel (a), but charge collection efficiency $\left[\eta_{\text {col }}\right]$ plotted against light intensity. Coloured dashed lines correspond to the limit set by the series resistance [see Eq. 5.7], while the thin, black solid line is a guide to the eye with a slope of $S=-1$ corresponding to theory explained above.

However, in the absence of an external applied voltage [at short-circuit], the maximum voltage drop across a device under illumination is $V_{\mathrm{dev}}=V_{\mathrm{oc}}$. Hence, the upper limit of the magnitude of the short-circuit current density as set by the series resistance is given by

$$
\left|J_{\mathrm{sc}}\right| \rightarrow \frac{V_{\mathrm{oc}}}{R_{\mathrm{S}}}
$$

Ideally, the open-circuit voltage scales with the light intensity as $q V_{\mathrm{oc}} \approx n_{\mathrm{id}} k_{\mathrm{B}} T \ln \left(I_{\mathrm{L}}\right)$. 


\section{LIGHT INTENSITY DEPENDENCE OF PHOTOCURRENT}

However, in reality $V_{\text {oc }}$ often saturates at high intensity. Ultimately, the short-circuit current tails off at such conditions [i.e., $\alpha \rightarrow 0$ ], with $\eta_{\text {col }}$ approaching an inverse light intensity dependence, manifest by a slope parameter of $S=-1$. This situation is depicted in Fig. 5.2 a [5.2 b], where the light intensity dependent $J_{\mathrm{sc}}\left[\eta_{\mathrm{col}}\right]$ is simulated for different series resistances varying between $R_{\mathrm{S}}=0 \Omega \mathrm{cm}^{2}$ and $R_{\mathrm{S}}=100 \Omega \mathrm{cm}^{2}$. The coloured dotted lines in $\mathbf{F i g}$. $5.2 \mathrm{~b}$ representing the theoretical limits estimated in accordance with Eq. $\mathbf{5 . 7}$ are in good agreement with the simulations. 


\subsection{The influence of space-charge effects}

The above considerations assume nearly balanced electron and hole transport, neglecting space charge effects. However, in a wide variety of organic solar cells imbalanced mobilities have been reported.131] As pointed out by Goodman and Rose, the photocurrent of a device under imbalanced mobility conditions is highly space-charge limited upon illumination at high light intensities due to the build-up of the carriers with the lower mobility, subsequently redistributing the internal electric field. 174 This is even more pronounced in systems with strongly suppressed recombination and imbalanced mobilities which can build up a large asymmetric charge density. 154

In general, when a significant amount of space charge is present in the active layer, e.g. caused by either doping or imbalanced mobilities, a considerable electric field redistribution will take place. This results in the formation of a space charge region, where the electric field is concentrated, and an electric field-free neutral region. In low-mobility systems, such as organic semiconductor-based light harvesting devices, exhibiting short diffusion lengths, the charge collection will only be efficient within the space charge region. For the case with mobility-induced space charge effects, the photocurrent can be approximated by 175

$$
J_{\mathrm{ph}} \approx-q G\left[w_{\mathrm{sc}}+L_{\mathrm{s}}\right]
$$

where $w_{\mathrm{sc}}=\sqrt{2 \varepsilon \varepsilon_{0}\left(V_{0}-V\right) /\left(q N_{\mathrm{sc}}\right)}$ and $L_{\mathrm{s}}=\sqrt{\mu_{\mathrm{s}} k_{\mathrm{B}} T \tau_{\beta} / q}$. Here, $N_{\mathrm{sc}}$ is the associated charge density [in the space-charge region] which for imbalanced mobilities is given by $N_{\mathrm{sc}} \approx \sqrt{\varepsilon \varepsilon_{0} G /\left(q \mu_{\mathrm{s}}\right)}$, while $V_{0}$ is the built-in potential across the space charge region. When the charge collection from the remainder of the active layer is assumed to be negligible, the short-circuit current simplifies to

$$
J_{\mathrm{ph}} \approx-q G^{3 / 4}\left[\frac{4 \mu_{\mathrm{s}} \varepsilon \varepsilon_{0}}{q}\right]^{1 / 4} \sqrt{V_{0}-V} .
$$

Subsequently, the photocurrent is expected to have an $\alpha=3 / 4$ power dependence on the photo-generation rate $G .176$. According to Eq. 5.4, this power dependence translates into a $S=-1 / 4$ power dependence when plotting the corresponding charge collection efficiency against the photo-generation rate. 


\section{LIGHT INTENSITY DEPENDENCE OF PHOTOCURRENT}
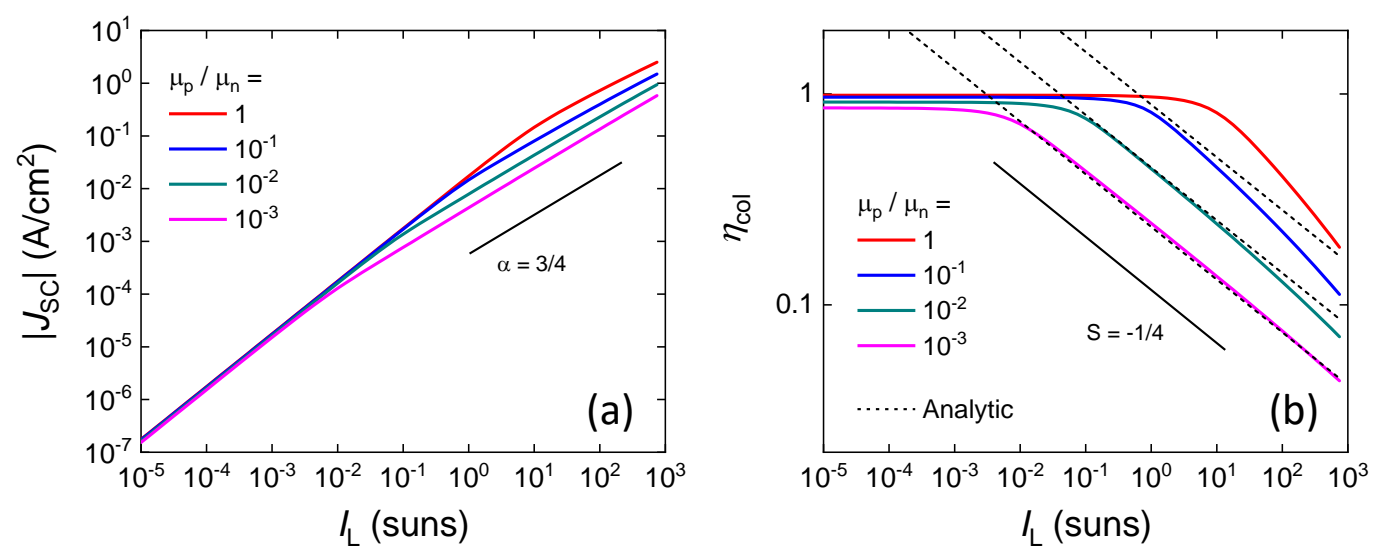

Figure 5.3: (a) Simulated short-circuit current density $\left[J_{\mathrm{sc}}\right]$ plotted as a function of light intensity $\left[I_{\mathrm{L}}\right]$, and compared for different imbalanced mobility ratios. When the photocurrent density is limited by space charge effects, a slope parameter of $\alpha=3 / 4$ is expected in accordance with above theory. (b) Charge collection efficiency $\left(\eta_{\text {col }}\right)$ plotted as a function of light intensity and simulated for the cases as presented in panel (a). For comparison, the analytically predicted charge collection efficiencies based on Eq. 5.9 have been included assuming $\beta=0.1 \beta_{\mathrm{L}}$.

Fig. 5.3a,b show the simulated short-circuit current density and the corresponding charge collection efficiency plotted as a function of light intensity, and compared for different degrees of imbalanced mobility ratios between $\mu_{\mathrm{p}} / \mu_{\mathrm{n}}=1$ and $\mu_{\mathrm{p}} / \mu_{\mathrm{n}}=10^{-3}$. Thin, black and solid lines are used as guides to the eye with a slope of $\alpha=3 / 4$, respectively $S=-1 / 4$. As shown in Fig. $\mathbf{5 . 3} \mathbf{a}, \mathbf{b}$, the onset point of deviation from the ideal, trap free case strongly depends on the degree of imbalanced mobility and shifts to lower intensity the more imbalanced the charge carriers. Dotted lines in Fig. 5.3 b are estimated in accordance with the analytical expression in Eq. 5.9 for the corresponding mobility ratios assuming $\beta=0.1 \beta_{\mathrm{L}}$ in all cases. 


\subsection{Recombination in the presence of trap states}

As previously described in Chapter 1, Section 1.8.2, when a significant amount of trap states are present in the bulk of the D-A blend, the recombination of charge carriers may also occur indirectly via those sub-gap states following first-order, trap-assisted Shockley-Read-Hall recombination [see Eq. 1.22]. Fig. 5.4a,b [c,d] shows the simulated short-circuit current density [charge collection efficiency] plotted as a function of light intensity for the case of acceptor-type [electron] trap states at varying trap depths [see Fig. 5.4a,c] and trap densities [see Fig. 5.4b,d]. At low light intensities, when $n \ll n_{1}$, or equivalently $E_{\mathrm{F}, \mathrm{n}}<E_{\mathrm{t}}$, the traps behave as shallow traps [see Chapter $\mathbf{1}$, Section 1.8.2. Under these conditions, corresponding to low carrier densities and/or shallow trap depth, $R_{\mathrm{SRH}}$ becomes effectively bimolecular, $R_{\mathrm{SRH}} \propto n p$. With the exception of the regions close to the contacts [where the back-injected carrier densities are high], the trap-assisted recombination within the active layer is expected to be weak in this intensity regime with the photocurrent being close to charge-carrier-recombinationfree, in analogy to the case in Section 5.3. The photocurrent thus follows a linear intensity dependence $J_{\text {sc }} \propto G^{\alpha}$ [with $\left.\alpha=1\right]$ manifested as a constant $\eta_{\text {col }}$ plateau. Note that if the traps are very close to the middle of the gap, this regime will be accessible only at extremely low light intensity and long time scales where it may be impractical to measure the photocurrent at low enough frequencies relevant to the long release time of deep traps.

At moderate intensities, in turn, when the carrier density in the active layer is eventually increased to such an extent that $n \gg n_{1}$ and $E_{\mathrm{F}, \mathrm{n}}>E_{\mathrm{t}}$, as such, the majority of the traps will be occupied by electrons. Under these conditions, the traps start to instead behave as deep traps and the trap-assisted recombination in the bulk is no longer negligible, becoming first-order $R_{\mathrm{SRH}} \sim n$. This will ultimately result in a second, down-shifted, linear photocurrent regime appearing as a second plateau in the EQE with a lower magnitude [compared to the first one] at moderate intensities. Subsequently, two different photocurrent [charge collection efficiency] regimes [plateaus] are expected under conditions when trap-assisted SRH recombination is the dominant photocurrent loss mechanism, with $n=n_{1}$ marking the point-of-transition [POT] between these two intensity regimes, as seen in Fig. 5.4. This unique feature allows for trap-assisted SRH recombination to be distinguished from other first-order losses. 


\section{LIGHT INTENSITY DEPENDENCE OF PHOTOCURRENT}

Note that exponential tail states would, in turn, induce a higher-order recombination channel with $\alpha<1$ [177, 178], hence leading to an EQE with $S<0$ at moderate light intensity [see Fig. B.6 in Appendix B].
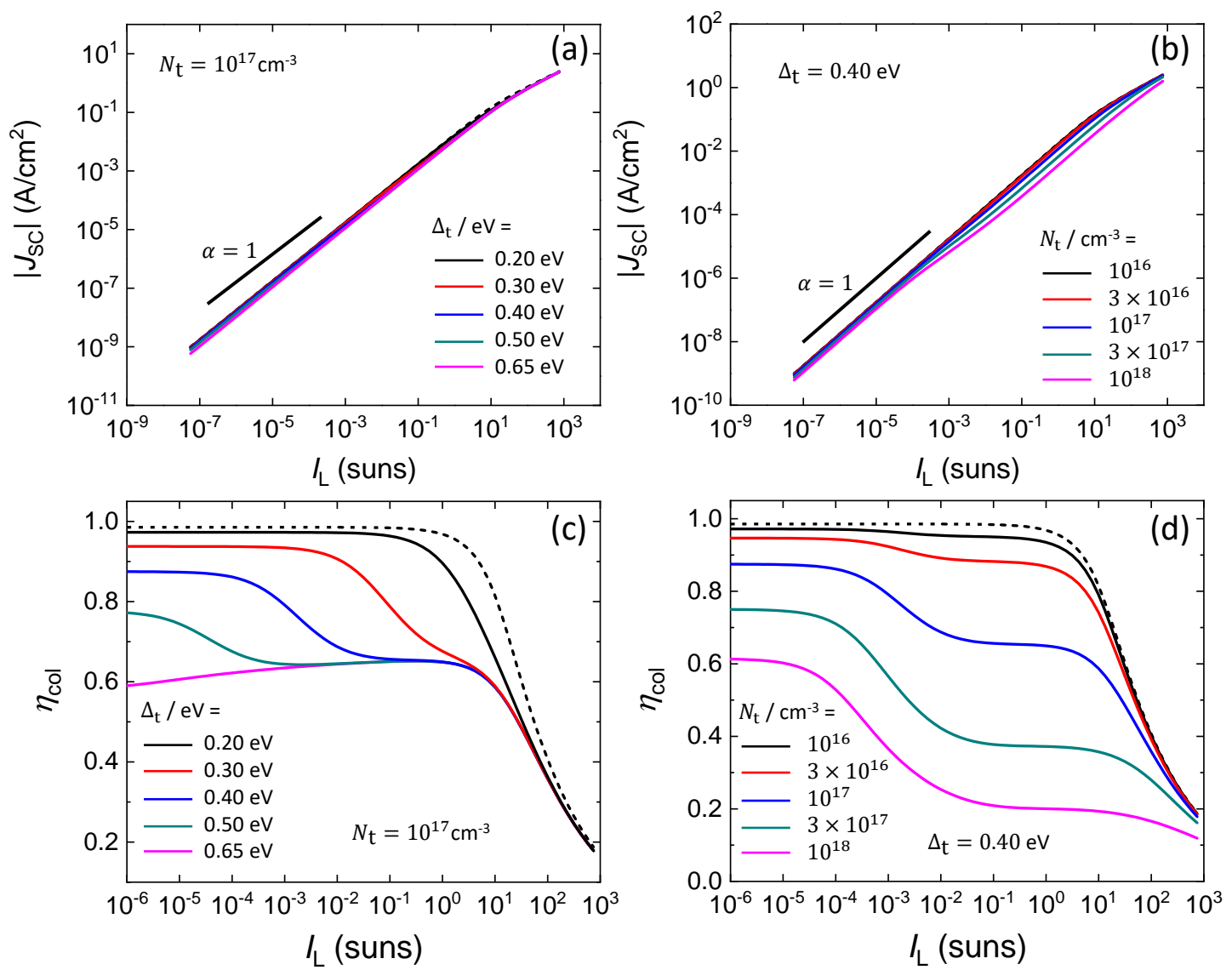

Figure 5.4: Simulated short-circuit current density $\left[J_{\mathrm{sc}}\right][$ top row $]$ and corresponding charge collection efficiency $\left[\eta_{\mathrm{col}}\right]$ [bottom row] in the presence of trap states in the D-A bulk for [a, c] trap depth $\Delta_{\mathrm{t}}$ varying between $0.2 \mathrm{eV}$ and $0.65 \mathrm{eV}$ for a constant trap density of $N_{\mathrm{t}}=10^{17} \mathrm{~cm}^{-3}$ and $[\mathbf{b}, \mathbf{d}]$ trap density $N_{\mathrm{t}}$ varying between $10^{16} \mathrm{~cm}^{-3}$ and $10^{18} \mathrm{~cm}^{-3}$ for a constant trap depth of $\Delta_{\mathrm{t}}=0.4 \mathrm{eV}$. Capture coefficients of $\beta_{\mathrm{n}}=\beta_{\mathrm{p}}=2 \times 10^{-11}$ $\mathrm{cm}^{3} \mathrm{~s}^{-1}$ were assumed in both cases.

In the moderate intensity regime, where first-order trap-assisted recombination dominates $\left[n \gg n_{1}\right]$, the recombination rate may be described by a constant lifetime for charge carriers. In accordance with the Hecht equation, thus a charge collection efficiency of the form given by Eq. 5.1 is expected. However, this equation is unable to reproduce the simulated $\eta_{\text {col }}$ values [at moderate intensities] in Fig. 5.4. This discrep- 
ancy can be traced back to the fact that the Hecht equation does not account for space charge effects. In the trap-filling limit, the occupied electron traps will behave as fixed negative charges. In analogy to Section $\mathbf{5 . 5}$ and the case with p-type doping [where the fixed negative charges are ionized dopants] [179], the electric field inside the active layer will be redistributed, mainly concentrated to a space charge region of trapped electrons [adjacent to the cathode]. Outside the space charge region, the active layer is charge-neutral [the trapped electrons are neutralized by injected/generated free holes] with the electric field being close to zero.

Under these conditions, the charge collection of photo-generated carriers is expected to be space-charge-limited with a photocurrent density of the form 179$]$ :

$$
J_{\mathrm{ph}} \approx-q G\left[w_{\mathrm{sc}}+L_{\mathrm{n}}\right]
$$

with

$$
w_{\mathrm{sc}}=\sqrt{\frac{2 \varepsilon \varepsilon_{0}\left[V_{\mathrm{bi}}-V\right]}{q N_{\mathrm{sc}}}}
$$

and

$$
L_{\mathrm{n}}=\sqrt{\frac{\mu_{\mathrm{n}} \tau k_{\mathrm{B}} T}{q}}
$$

being the space charge region thickness and electron diffusion length in the chargeneutral region, respectively, where $N_{\mathrm{sc}}$ is the space charge density [of trapped electrons] in the space charge region, $V_{\mathrm{bi}}$ is the associated built-in potential, and $1 / \tau=\beta_{\mathrm{n}} N_{\mathrm{t}}$. Assuming traps to be, on average, half-filled within the space charge region, the space charge density in this region may be approximated by $N_{\mathrm{sc}} \approx N_{\mathrm{t}} / 2$. Accordingly, the collection efficiency at short-circuit can be expressed as

$$
\eta_{\mathrm{col}} \approx \sqrt{\frac{\varepsilon \varepsilon_{0} k_{\mathrm{B}} T}{q^{2} d^{2} N_{\mathrm{t}}}}\left[2 \sqrt{\frac{q V_{\mathrm{bi}}}{k T}}+\sqrt{\zeta_{\mathrm{n}}}\right]
$$

with $\zeta_{\mathrm{n}}=\beta_{\mathrm{n}} \times\left[q \mu_{\mathrm{n}} /\left(\varepsilon \varepsilon_{0}\right)\right]^{-1}$ being a recombination pre-factor of a corresponding Langevin recombination coefficient based on the electron mobility. 


\section{LIGHT INTENSITY DEPENDENCE OF PHOTOCURRENT}

\subsubsection{Trap depth estimation via point-of-transition approach}

The trap depth can be estimated from the point-oftransition [POT] between the low intensity regime, where first-order $\mathrm{SRH}$ recombination in the bulk is negligible, and the moderate intensity regime where first-order SRH recombination dominates [trap-filling limit]. Here, a device with an active layer containing electron traps [acceptor-type] is considered [the case with hole traps is analogous]. At low intensities [when recombination and space charge effects are negligible], the associated average electron density $n_{\text {low }}$ is related to the total short-circuit current $J_{\text {sc }}$ via

$$
J_{\mathrm{sc}}=2 q n_{\mathrm{low}} \mu_{\mathrm{n}} F=\frac{2 q n_{\mathrm{low}} d}{t_{\mathrm{tr}}},
$$

where $t_{\mathrm{tr}}$ is the electron transit time $\left[\mu_{\mathrm{n}}\right.$ is the electron mobility and $F$ the electric field]. At these intensities, it is also expect that $J_{\mathrm{sc}} \approx J_{\mathrm{G}}$, where $J_{\mathrm{sc}}$ is the photogeneration current. POT occurs when $n_{\text {low }} \approx n_{1}$ [see Section 5.6]; making use of the definition of $n_{1}$ [see Chapter 1, Section 1.8.2], it then follows that

$$
\Delta_{\mathrm{t}}=k_{\mathrm{B}} T \ln \left(\frac{2 q N_{\mathrm{L}, \mathrm{A}} d}{J_{\mathrm{G}, \text { РОT }} t_{\mathrm{tr}}}\right)
$$

allowing for the trap depth $\Delta_{\mathrm{t}}$ to be determined. In Fig. 5.5, the POT approach to determine the trap depth is demonstrated on a PM6:BTP-eC9 solar cell. Fig. 5.5a,b,c show the short-circuit current density,

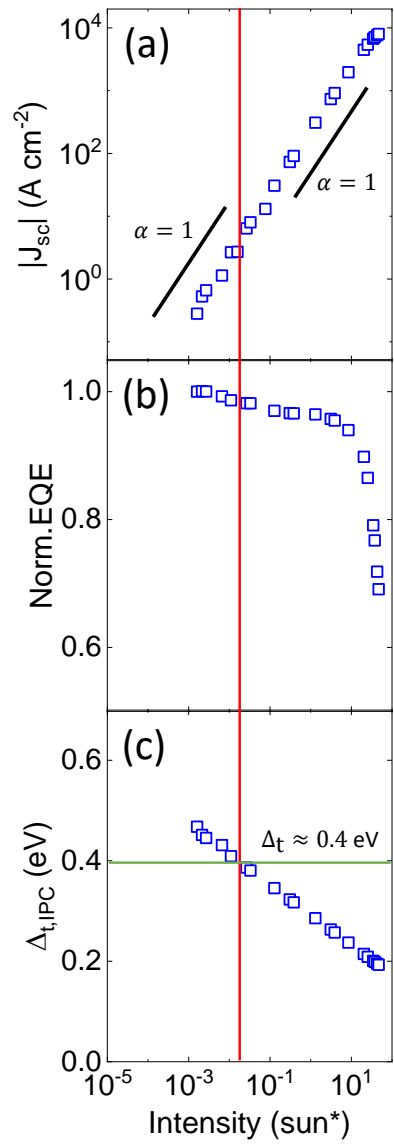

Figure 5.5: (a) $J_{\mathrm{sc}}, \quad$ (b) normalized EQE and (c) $\Delta_{\mathrm{t}}$ [estimated via the pointof-transition approach] of PM6:BTP-eC9 solar cell $\left(d=100 \mathrm{~nm}, \quad V_{\text {bias }}=0 \mathrm{~V}\right.$, $\lambda_{\text {exc }}=520 \mathrm{~nm}$ ); the red vertical line marks the point-oftransition light intensity. normalized external quantum efficiency [EQE], and calculated trap depth plotted as a function of light intensity. A two-step EQE behaviour in the low and moderate intensity regime is observed, from which the POT light intensity [Fig. 5.5; vertical red line] is defined. The normalized EQE was calculated from the raw IPC data in accordance with Eq. 5.2 and a trap depth of $\Delta_{\mathrm{t}}=0.41 \mathrm{eV}$ was determined, respectively. 


\subsubsection{Trap depth estimation via quasi-Fermi level splitting approach}

The energy of the trap states, located within the donor-acceptor bandgap $E_{\mathrm{g}}$ [with $E_{\mathrm{g}}=\left|E_{\mathrm{H}, \mathrm{D}}-E_{\mathrm{L}, \mathrm{A}}\right|$, where $E_{\mathrm{H}, \mathrm{D}}$ denotes the HOMO energy level of the organic donor and $E_{\mathrm{L}, \mathrm{A}}$ denotes the LUMO energy level of the organic acceptor], can be determined if the point-of-transition [POT] is known. Assuming a symmetric quasi-Fermi level splitting $E_{\mathrm{QFLS}}$ at the POT [i.e., $E_{\mathrm{QFLS}}=\left|E_{\mathrm{F}, \mathrm{n}}-E_{\mathrm{F}, \mathrm{p}}\right|$ around the mid-gap of the organic D-A BHJ], the trap depth [relative to the HOMO energy level of the donor, respectively LUMO energy level of the acceptor] can be estimated from

$$
\Delta_{\mathrm{t}, \mathrm{QFLS}}=\frac{E_{\mathrm{g}}-E_{\mathrm{QFLS}}}{2}=\frac{\left|E_{\mathrm{H}, \mathrm{D}}-E_{\mathrm{L}, \mathrm{A}}\right|}{2}-\frac{q V_{\mathrm{oc}}}{2} .
$$

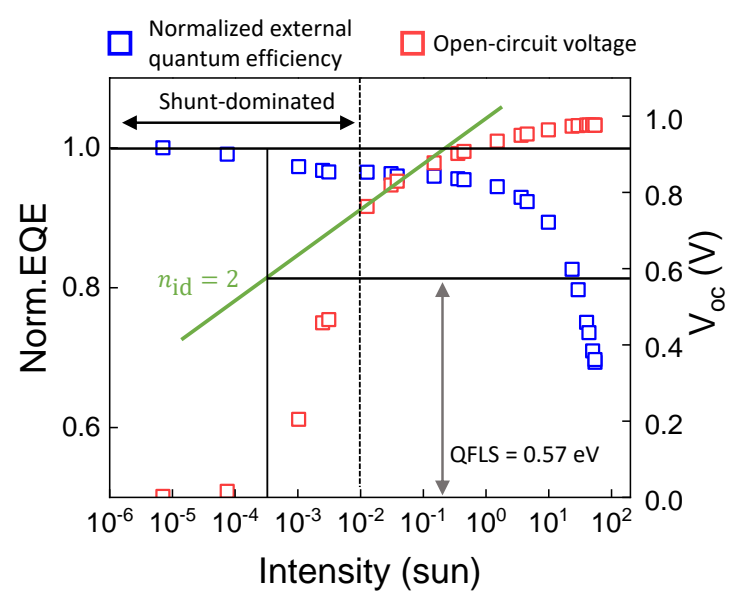

Figure 5.6: Normalized EQE [left axis] and $V_{\text {oc }}$ [right axis] of a PM6:BTP-eC9 solar cell [see Appendix A for device fabrication details] plotted as a function of intensity. The solid [dashed] vertical black line marks the point-of-transition intensity between the two EQE plateaus [shunt-dominated $V_{\text {oc }}$-regime]. A quasi-Fermi level splitting [trap depth] was estimated to $0.57 \mathrm{eV}\left[\Delta_{\mathrm{t}, \mathrm{QFLS}}=0.41 \mathrm{eV}\right]$ from the would-be shunt-free $V_{\text {oc }}$ corresponding to an light ideality factor of $n_{\text {id }}=2$ [green line].
Provided that surface recombination is negligible, the $V_{\text {oc }}$ of an organic solar cell gives a direct estimate of the quasiFermi level splitting $E_{\mathrm{QFLS}}=q V_{\mathrm{Oc}}$ in the bulk. However, at low light intensities, the $V_{\text {oc }}$ [and subsequently the determination of $\left.E_{\text {QFLS }}\right]$ becomes limited by the shunt resistance of the device. In such cases an extrapolation of the $V_{\text {oc }}$ from high to low intensity may be used to estimate a would-be shuntfree $V_{\text {oc }}$ at lower light intensities in order to obtain $E_{\text {QFLS }}$. The quasi-Fermilevel approach to estimate $\Delta_{\mathrm{t}, \mathrm{QFLS}}$ is demonstrated on a PCDTBT:PC ${ }_{70} \mathrm{BM}$ solar cell [see Fig. 5.6]. Here, red [blue] symbols correspond to the experimentally obtained $V_{\mathrm{oc}}$ [normalized EQE] data; the vertical black dotted

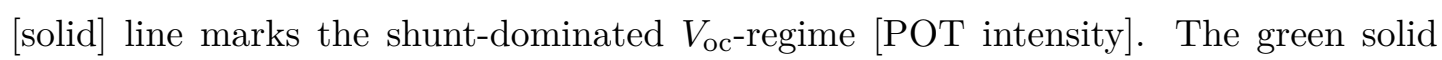
line is a guide to the eye corresponding to an ideality factor of $n_{\text {id }}=2$ from which the 'would-be', shunt-free $V_{\text {oc }}$ was estimated [see horizontal black line in Fig. 5.6. 


\section{LIGHT INTENSITY DEPENDENCE OF PHOTOCURRENT}

\subsubsection{Trap density estimation via space-charge approach}

To obtain an order of magnitude estimate of the trap density $N_{\mathrm{t}}$, in turn, the moderate intensity regime is considered. In this regime, the occupied traps will behave as fixed negative charges. In analogy to the case with p-type doping [where the fixed negative charges are ionized dopants][179], the electric field $F$ inside the active layer will be mainly concentrated to a space charge region of trapped electrons [adjacent to the cathode], while the remaining active layer is neutral [the trapped electrons are neutralized by injected/generated free holes] with $F \approx 0$. Then, assuming that the charge extraction of photo-generated carriers is predominantly taking place from the space charge region, the charge collection efficiency under short-circuit conditions is expected to take the approximate form $[179]$ :

$$
\eta_{\mathrm{col}} \sim \frac{w_{\mathrm{sc}}}{d}
$$

where

$$
w_{\mathrm{sc}}=\sqrt{\frac{2 \varepsilon \varepsilon_{0} V_{\mathrm{bi}}}{q N_{\mathrm{sc}}}}
$$

is the space charge region thickness and $d$ is the active layer thickness. Here, $N_{\mathrm{sc}}$ is the space charge density [of trapped electrons] within the space charge region, while $V_{\mathrm{bi}}$ is the associated built-in potential. Assuming $N_{\mathrm{sc}} \approx N_{\mathrm{t}}$, and noting that $\eta_{\mathrm{col}} \approx \mathrm{EQE}_{\text {norm }}$, the following expression for estimating the trap density is obtained:

$$
N_{\mathrm{t}}=\frac{2 \varepsilon \varepsilon_{0} V_{\mathrm{bi}}}{q d^{2}} \times\left[\frac{1}{\mathrm{EQE}_{\mathrm{norm}}}\right]^{2},
$$

where $\mathrm{EQE}_{\text {norm }}$ is the EQE of the second $\mathrm{EQE}$ plateau at moderate irradiance normalized to the EQE plateau at low irradiance with higher magnitude. 


\subsection{Simplified analysis for general recombination order}

In the previous Sections $5 \mathbf{5 . 2}$ - 5.6 the interplay between first and second-order recombination kinetics has mainly considered. However, these considerations may also be qualitatively extended to the case with a general recombination order $\sigma$ for photo-generated charge carriers. The corresponding recombination rate is of the form $R=B n^{\sigma}$, with the associated recombination coefficient given by $B$. In this case, assuming space charge effects to be negligible, the steady-state charge extraction of carriers at short-circuit can, in approximate terms, be described by

$$
\frac{d n}{d t}=G-\frac{n}{t_{\mathrm{tr}}}-B n^{\sigma}=0
$$

where $t_{\text {tr }}$ is the extraction time. In this simplified analysis, the short-circuit current is given by $J_{\mathrm{sc}}=-q d \frac{n}{t_{\mathrm{tr}}}$. It then follows that at small intensities [small $n$ ], the extraction term dominates [all carriers are extracted] such that $J_{\mathrm{sc}}=-q G d$, as expected. At high intensities, when the recombination term dominates, in turn, $n \approx(G / B)^{1 / \sigma}$ is obtained, and thus $J_{\mathrm{sc}} \propto G^{1 / \sigma}$, corresponding to slopes of $\alpha=1 / \sigma$ and $S=(1-\sigma) / \sigma$. Hence, under conditions when third-order recombination kinetics $[\sigma=3]$, expected in conjunction with Auger recombination, dominates, a slope of $\alpha=1 / 3$ and $S=-2 / 3$ would be observed. 


\section{LIGHT INTENSITY DEPENDENCE OF PHOTOCURRENT}

\subsection{Sensitive IPC of thin-film, organic solar cells}

To validate the theoretical framework, experimental IPC measurements on organic solar cells were conducted. The IPC measurements were performed at an excitation wavelength of $520 \mathrm{~nm}$ and no bias voltage [short-circuit] was applied to the devices. Further experimental details of the IPC measurements are provided in Box 5.1. From the raw IPC data the corresponding EQE versus light intensity were calculated according to Eq. 5.2 with the charge collection efficiency being reflected by the EQE via Eq. 5.4. A $110 \mathrm{~nm}$ thick PM6:ITIC and PBDB:T:EH-IDTBR system were selected as model systems to demonstrate the different IPC regimes discussed above; details to device fabrication are provided in Appendix $\mathbf{A}$ and light $J-V$ characterization can be found in Fig. 6.3 in Chapter 6. The PBDB-T:EH-IDTBR was specifically selected to demonstrate a device with imbalanced mobilities. In accordance with above explanations different photocurrent loss mechanisms can be identified and will be briefly discussed in the following.
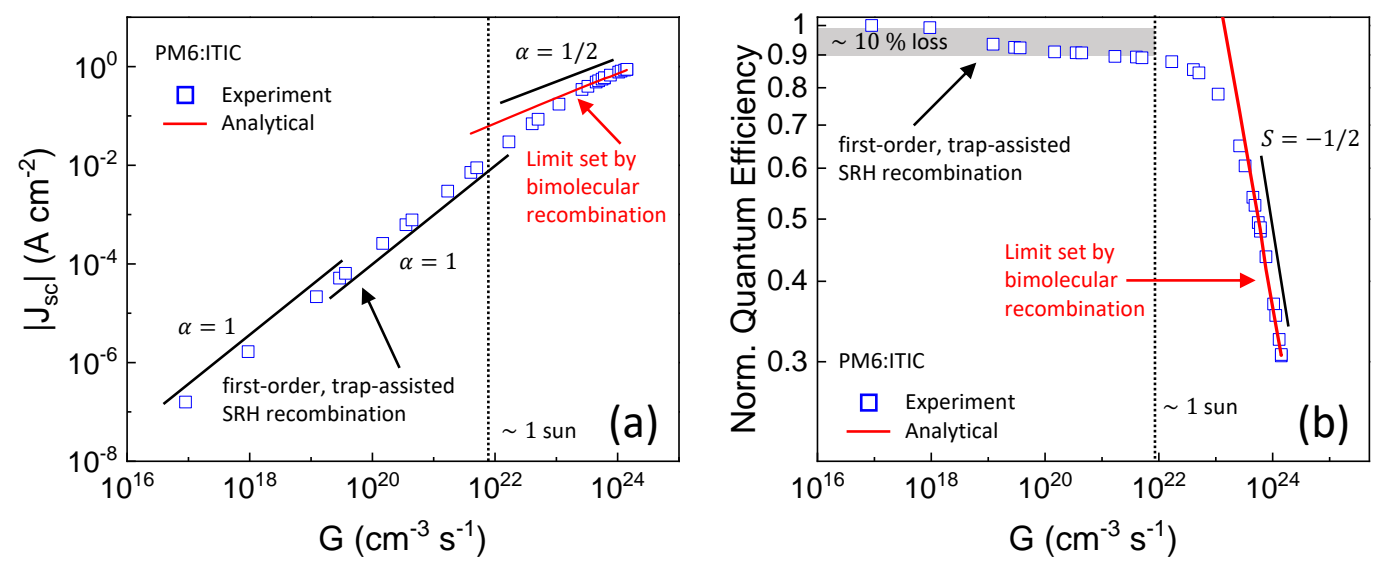

Figure 5.7: (a) Experimentally obtained short-circuit current density $\left[J_{\mathrm{sc}}\right]$ and (b) normalized external quantum efficiency [EQE] of a PM6:ITIC solar cell measured sensitively over a broad range of intensity. The red solid lines in (a) and (b) represent the limit set by a bimolecular recombination in accordance with Eq. 5.5 assuming $\mu_{\mathrm{n}}=3 \times 10^{-4}$ $\mathrm{cm}^{2} \mathrm{~V}^{-1} \mathrm{~s}^{-1}$ and $\mu_{\mathrm{p}}=3 \times 10^{-5} \mathrm{~cm}^{2} \mathrm{~V}^{-1} \mathrm{~s}^{-1}, d=110 \mathrm{~nm}, \beta=1.5 \times 10^{-11} \mathrm{~cm}^{3} \mathrm{~s}^{-1}$ and $\varepsilon=3.5$. Black solid lines are used as guides to the eye with a slope corresponding to the predominant photocurrent loss mechanism and the theory explained above [see Section $5.2-5.6$. 
Experimental details of steady-state light intensity dependent photocurrent (IPC) measurements

\section{Box 5.1}

A commercial laser with variable output power was used as a light source and modulated at a low frequency $[<0.1 \mathrm{~Hz}]$ using a Function Generator [Keysight Technology, 33500B Series]. The light perturbation allowed for precise and noisereduced measurements of the external photocurrent. Here, the choice of modulation frequency $f$ was limited by both the technical aspects of the intensity dependent photocurrent [IPC] setup itself, and the physical processes governing the device under test [DUT] [e.g., charge carrier transport, (de-) trapping time and RC-time of the circuit]. A motorized two-wheel attenuator [Standa, 10MCWA168-1], containing different optical density [OD] filters, was used to additionally vary the input light intensity. The combination of both variable laser output power and two-wheel attenuator together with a low frequency photocurrent modulation allowed for sensitive measurements of the photocurrent over multiple orders of magnitude light intensity. In order to avoid partial illumination of the active area of the DUT and thus, the formation of recombination centers due to the local accumulation of photogenerated charge carries, the laser light profile was homogenized by fiber-coupling the excitation light into an integrating sphere [Thorlabs, IS236A-4] prior illumination of the DUT active layer. Mounted on the integrating sphere, a Silicon photodiode [Thorlabs, SM05PD1A] was used for light power calibration and in situ intensity tracking. Two Keithley source-measure units [Keithley 2450] were used to simultaneously read the DUT photocurrent [density] and the Silicon photodiode current, while different bias voltages can be applied on the DUT. For the initial calibration process, an additional NIST ${ }^{1}$-calibrated Silicon photodiode power sensor [Thorlabs, S121C] was used.

Fig. 5.7 a,b and Fig. 5.8 a,b show the short-circuit current density and the normalized EQE, respectively, of the PM6:ITIC and PBDB-T:EH-IDTBR solar cell measured sensitively over a broad range of light intensities. At low incident light intensities [i.e. up to $G \approx 10^{18} \mathrm{~cm}^{-3} \mathrm{~s}^{-1}$, both the PM6:ITIC and PBDB-T:EH-IDTBR solar cells exhibit a linear photocurrent regime with a slope of $\alpha=1$ [see black solid line in Fig. 5.7a and Fig. 5.8 a] which directly translates into a constant EQE plateau [see Fig. 5.7 b and Fig. 5.8 $\mathbf{b}$ ]. The EQEs were normalized suggesting that approximately $25 \%$ [35\%] of the PM6:ITIC [PBDB-T:EH-IDTBR] EQEs are lost due to inefficient photon absorp-

\footnotetext{
${ }^{1}$ National Institute of Standards and Technology
} 


\section{LIGHT INTENSITY DEPENDENCE OF PHOTOCURRENT}

tion and charge generation. At moderate intensities [i.e., $10^{18}<G<10^{21} \mathrm{~cm}^{-3} \mathrm{~s}^{-1}$ ], a slightly down-shifted, second photocurrent regime [equivalent EQE plateau], can be observed in both devices indicating the presence of trap states in the D-A active layer bulks. We note that the observed two-step EQE behaviour does not rule out the presence of exponential tail states, but instead, indicates SRH recombination via deep trap states to be the dominant photocurrent loss channel at the given light intensity regime.
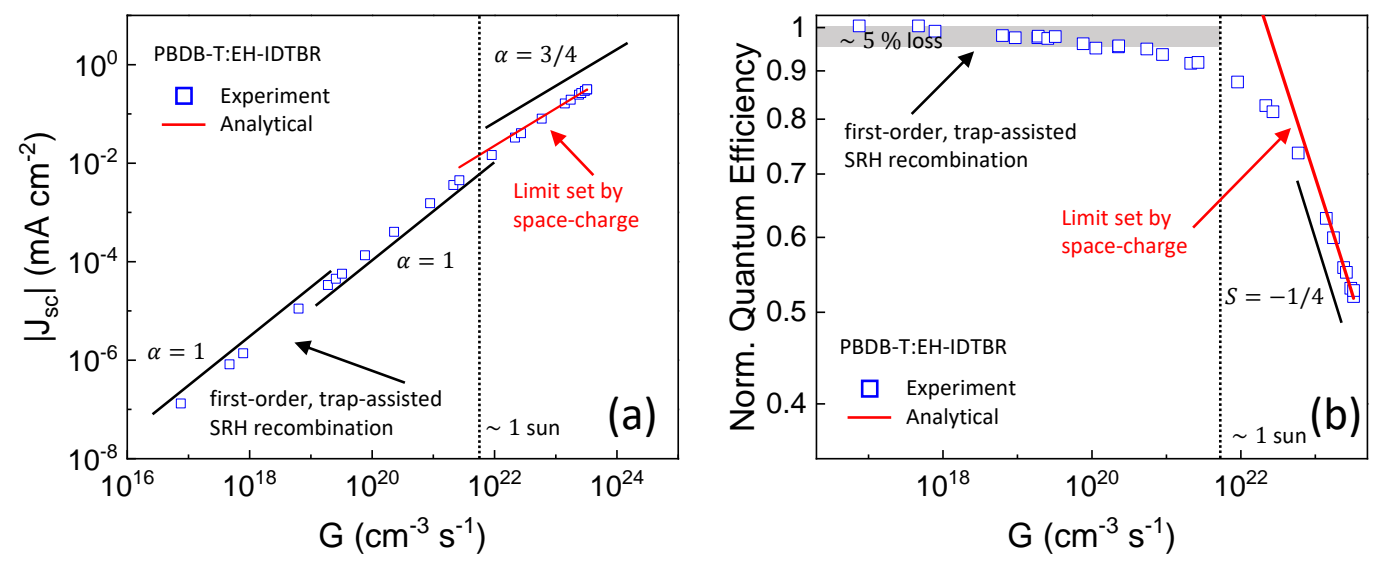

Figure 5.8: (a) Experimentally obtained short-circuit current density $\left[J_{\mathrm{sc}}\right]$ and (b) normalized external quantum efficiency [EQE] of a PBDB-T:EH-IDTBR solar cell measured sensitively over a broad range of intensity. The red solid lines in (a) and (b) mark the space-charge-limited photocurrent limit determined in accordance with Eq. $\mathbf{5 . 9}$ assuming $\mu_{\mathrm{p}}=8.1 \times 10^{-7} \mathrm{~cm}^{2} \mathrm{~V}^{-1} \mathrm{~s}^{-1}, d=110 \mathrm{~nm}$ and $\varepsilon=3.5$. Black solid lines are used as guides to the eye with a slope corresponding to the predominant photocurrent loss mechanism and the theory explained above [see Section $5.2-5.6]$.

At intensities above 1 sun $\left[\sim 5 \times 10^{21} \mathrm{~cm}^{-3} \mathrm{~s}^{-1}\right]$, bimolecular recombination in the PM6:ITIC device starts to dominate over charge extraction leading to a photocurrent [EQE] versus generation rate power dependence of $\alpha=1 / 2[S=-1 / 2]$, in accordance with Eq. 5.5. The corresponding limits set by bimolecular recombination based on Eq. 5.5 is indicated by the red solid lines in Fig. 5.7 a and Fig. 5.7 b for the short-circuit current density and normalized EQE. As shown, the estimated limits are in agreement with the experimental data assuming an electron and hole mobility of $\mu_{\mathrm{n}}=3 \times 10^{-4}$ $\mathrm{cm}^{2} \mathrm{~V}^{-1} \mathrm{~s}^{-1}$ and $\mu_{\mathrm{p}}=3 \times 10^{-5} \mathrm{~cm}^{2} \mathrm{~V}^{-1} \mathrm{~s}^{-1}$ as well as a dielectric constant of $\varepsilon=3.5$ and a bimolecular recombination coefficient of $\beta=1.5 \times 10^{-11} \mathrm{~cm}^{3} \mathrm{~s}^{-1}$.

The PBDB-T:EH-IDTBR device, in turn, suffers at moderate and high intensities [i.e., $10^{-3}<I_{\mathrm{L}}<1 \mathrm{Wcm}^{-2}$ ] from photocurrent losses due to imbalanced carrier mobilities 
and the build-up of space charge. The red solid lines in Fig. 5.8 a and Fig. 5.8 b mark the limit set by the imbalanced mobilities estimated in accordance with Eq. 5.9 assuming a slower carrier mobility of $\mu_{\mathrm{s}}=8.1 \times 10^{-7} \mathrm{~cm}^{2} \mathrm{~V}^{-1} \mathrm{~s}^{-1}$ and a dielectric constant of $\varepsilon=3.5$. In accordance with Eq. 5.15 from Section 5.6.1, the trap depths for both devices can be obtained at the corresponding point-of-transition [POT] intensity. For the PM6:ITIC device a trap depth of $\Delta_{\mathrm{t}}=0.4 \mathrm{eV}$ was obtained, while for the PBDB-T:EH-IDTBR device a trap depth of $\Delta_{\mathrm{t}}=0.45 \mathrm{eV}$ was estimated. In both cases $N_{\mathrm{L}, \mathrm{A}}=10^{20} \mathrm{~cm}^{-3}$ was assumed and $t_{\mathrm{tr}}=6 \times 10^{-7} \mathrm{~s}\left[t_{\mathrm{tr}}=1 \times 10^{-7} \mathrm{~s}\right]$ were determined for PM6:ITIC [PBDB-T:EH-IDTBR] from RPV [details to the RPV technique are provided in Box 3.3; experimentally obtained RPV transients can be found in Fig. B.5 in Appendix B. In this regard, an estimate of the corresponding trap densities can be obtained based on Eq. $\mathbf{5 . 1 9}$ from Section 5.6.3. Assuming $V_{\mathrm{bi}} \approx 1 \mathrm{~V}$ for the PM6:ITIC and PBDB-T:EH-IDTBR devices, trap densities for both devices was found to be on the order of $\sim 10^{17} \mathrm{~cm}^{-3}$. It is worth noting that the exact origin of the observed trap states remains unknown. The obtained trap depths are, however, in agreement with those recently reported by Zuo et al. $\left[0.3 \leq \Delta_{\mathrm{t}} \leq 0.6 \mathrm{eV}\right]$ for single-carrier devices pointing towards water-induced trap states. 180. 


\section{LIGHT INTENSITY DEPENDENCE OF PHOTOCURRENT}

\subsection{Conclusion}

In this chapter, a detailed study of charge collection efficiency as a function of light intensity has been presented. As a result, the influence of different photocurrent loss mechanisms has been clarified. It should be stressed that a certain photocurrent loss mechanism can be only identified as such if the photocurrent density [charge collection efficiency] versus light intensity unambiguously follows the power dependence as predicted by the above theory. Since predominant photocurrent loss mechanisms vary with intensity and transition regimes occur in between them, it is of the utmost importance when determining the predominant loss mechanism to measure the $J_{\mathrm{sc}}, \eta_{\mathrm{col}}$ [or EQE], over a broad range of light intensities. Failing to do so, will lead to a misjudgment of the predominant photocurrent loss mechanisms and the corresponding recombination order. In this regard, it should also be emphasized that a general deviation from linearity [i.e., $\alpha<1$, when plotting $J_{\mathrm{sc}}$ versus $I_{\mathrm{L}}$, or $S<0$, when plotting $\eta_{\text {col }}$ versus $I_{\mathrm{L}}$ ], does not automatically imply the dominating presence of second-order [bimolecular] recombination, and other photocurrent loss mechanisms, for instance imbalanced mobilities and the build-up of space charge, device series resistance and trap-assisted recombination, have to be taken into account. Especially in the case of the latter one, the deviation could turn out to be merely the transition between two linear [constant] $J_{\mathrm{sc}}\left[\eta_{\mathrm{col}}\right]$ regimes pointing towards a first-order recombination process and the presence of trap states.

Tab. 5.1 in Box 5.2 summarizes the above recombination and photocurrent loss mechanisms [i.e., ideal and loss-free charge collection, first-order absorption and generation loss, first-order, trap-assisted SRH recombination, second-order, bimolecular recombination, imbalanced mobility transport, series resistance limitation and trimolecular Auger recombination] with particular focus on their signatures in steady-state IPC measurements and the corresponding $J_{\mathrm{sc}}$ versus $I_{\mathrm{L}}$, and EQE versus $I_{\mathrm{L}} \log$-log plots [see Fig. 5.9]. In this regard, it is worth noting that it is also common in the literature to plot the EQE versus photocurrent [density] instead of light intensity. [131, 154, 170] In this case the expected slopes for the photocurrent loss mechanisms will change accordingly [see Tab. 5.1]. 
Summary of recombination and photocurrent loss mechanism signatures in steady-state IPC measurements

Box 5.2

\begin{tabular}{|c|c|c|c|}
\hline \multirow{2}{*}{ Mechanism } & \multicolumn{3}{|c|}{ Slope in a log-log plot } \\
\hline & $J_{\text {sc }}$ versus $I_{\mathrm{L}}$ & EQE versus $I_{L}$ & EQE versus $J_{\mathrm{sc}}$ \\
\hline ideal charge collection & 1 & 0 & 0 \\
\hline absorption and generation loss & 1 & 0 & 0 \\
\hline bimolecular recombination & $1 / 2$ & $-1 / 2$ & -1 \\
\hline $\begin{array}{l}\text { trap-assisted Shockley-Read-Hall } \\
(\mathrm{SRH}) \text { recombination }\end{array}$ & $\begin{array}{c}\text { two shifted and unity } \\
\text { slope regimes }\end{array}$ & $\begin{array}{l}\text { two shifted and } \\
\text { constant plateaus }\end{array}$ & $\begin{array}{l}\text { two shifted and constant } \\
\text { plateaus }\end{array}$ \\
\hline space-charge & $3 / 4$ & $-1 / 4$ & $-1 / 3$ \\
\hline series resistance & $J_{\mathrm{sc}} \propto \ln \left(I_{\mathrm{L}}\right)$ & -1 & $\begin{array}{l}\text { Asymptotically convergent to } \\
\qquad J_{\mathrm{sc}} \approx V_{\mathrm{oc}} / \mathrm{R}_{\mathrm{s}}\end{array}$ \\
\hline trimolecular Auger recombination & $1 / 3$ & $-2 / 3$ & -2 \\
\hline
\end{tabular}

Table 5.1: Expected slopes in a log-log plot for relations between short-circuit current density $\left[J_{\mathrm{sc}}\right]$, external quantum efficiency $[\mathrm{EQE}]$ and light intensity $\left[I_{\mathrm{L}}\right]$ when different photocurrent loss mechanisms are predominant.
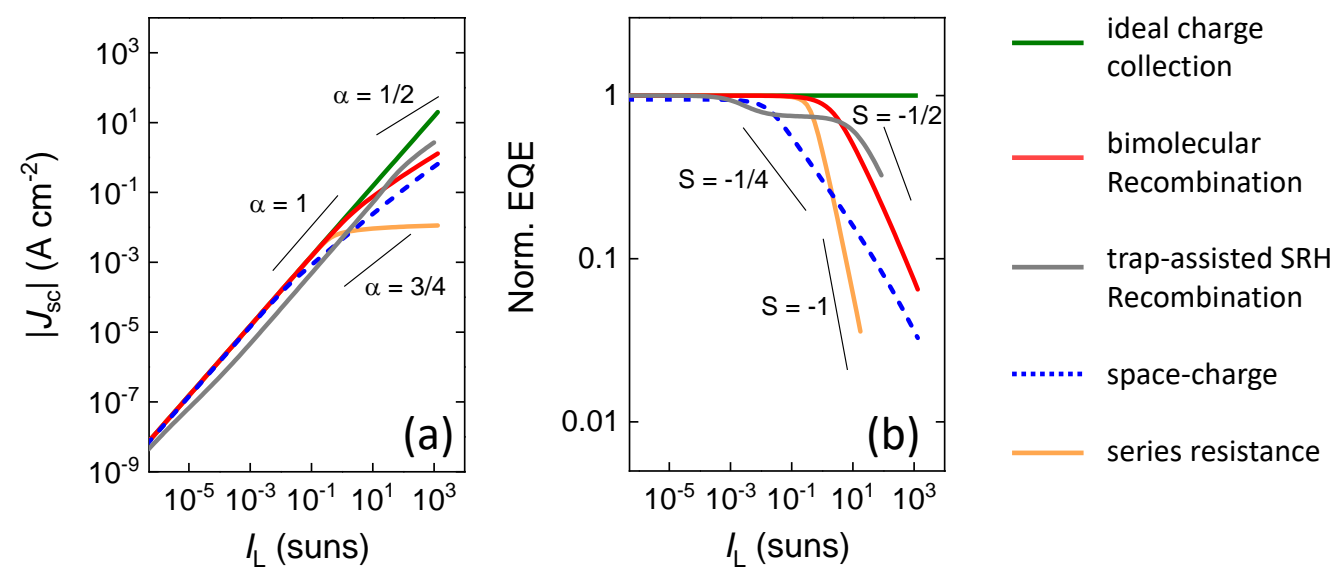

Figure 5.9: (a) Simulated short-circuit current density $\left[J_{\mathrm{sc}}\right]$ and (b) normalized external quantum efficiency $[\mathrm{EQE}]$ plotted as a function of light intensity $\left[I_{\mathrm{L}}\right]$, and compared for different photocurrent loss mechanisms. 
5. LIGHT INTENSITY DEPENDENCE OF PHOTOCURRENT 


\section{Chapter 6}

\section{Direct observation of trap-assisted recombination}

Trap-assisted recombination caused by localised sub-gap states is one of the most important first-order loss mechanisms limiting the power-conversion efficiency of all types of solar cells. The presence and relevance of trap-assisted recombination in organic photovoltaic devices is still a matter of some considerable ambiguity and debate, hindering the field as it seeks to deliver ever higher efficiencies and ultimately a viable new solar photovoltaic technology. In this chapter it is shown that trap-assisted recombination loss of photocurrent is universally present under operational conditions in a wide variety of organic solar cell materials including the new non-fullerene electron acceptor systems currently breaking all efficiency records for organic solar cells. The traps are found to consist of deep states lying $0.35-0.6 \mathrm{eV}$ below the transport edge. Apart from limiting the photocurrent, it is shown that trap-assisted recombination via these comparatively deep traps is also responsible for ideality factors between 1 and 2 , shedding further light on another open and important question as the fundamental working principles of organic solar cells.

This chapter is based upon a collaborative work published in 2021 by the author in the journal Nature Communications. 153] 


\section{DIRECT OBSERVATION OF TRAP-ASSISTED RECOMBINATION}

\subsection{Trap states in organic semiconductors}

Recently, organic solar cells have surpassed $17 \%$ [11], 181] power conversion efficiency $[\mathrm{PCE}]$ in single-absorber layer bulk heterojunction [BHJ] devices based upon nonfullerene electron acceptor [NFA] systems. This represents a major advance in the field, and indeed may be the catalyst to move organic solar cells to 'viable technology' status. BHJs have also been used in state-of-the-art photodetectors such as photodiodes with wide dynamic range, [96] high specific detectivities [182, 183] and colour selectivity. [184] In order to further optimize the device performance of organic photovoltaic devices, including organic solar cells and photodetectors, a better understanding of fundamental processes limiting the photocurrent and thereby the power conversion efficiency, is a pre-requisite. In state-of-the-art solar cells, the short-circuit current is limited by first-order losses, including those due to non-optimal absorption and geminate recombination of excitons and charge transfer [CT] states. Another first-order loss mechanism is recombination via trap states - that is, through available states for charge carriers within the energy gap. In general, traps and the associated trap-assisted recombination also give rise to increased non-radiative photovoltage losses and reduced fill factors. While losses caused by absorption and geminate recombination have been studied in detail and can be directly linked to material properties, the presence and role of traps remain controversial topics in organic solar cells and organic optoelectronics more broadly.

In neat organic devices, universal traps at $3.6 \mathrm{eV}$ and $6.0 \mathrm{eV}$ [below the vacuum level] limiting carrier transport have been observed, attributed to water-oxygen complexes and water clusters, respectively. 185, 186] These traps define an energetic window for bipolar trap-free charge transport in organic semiconductors: if the lowest unoccupied molecular orbital [LUMO] [or highest occupied molecular orbital (HOMO)] lies above [below] the electron [hole] trap level, the electron [hole] transport is inevitably traplimited. This explains the unipolarity of long-range charge transport in most organic semiconductors at low carrier densities. 184, 187. A different conclusion was reached in a recent study by Zuo et al. who suggested that the traps are caused by water-filled nanovoids, inducing trap levels that are always approximately $0.3-0.4 \mathrm{eV}$ above [below] the HOMO [LUMO]. 180 ] 
In BHJs, the LUMO of the electron-transporting acceptor and the HOMO of the holetransporting donor typically lie within the window of trap-free electron and hole transport in their respective domains. Despite this, the presence of traps [and trap-assisted recombination] has been frequently reported for organic BHJ solar cells. 136, 169, 188 193. Whether these traps are deep gap states or shallow tail states below the transport level is a heavily debated subject. Furthermore, the actual trap-induced losses under realistic operating conditions [i.e., irradiances of $\sim 1$ sun and at maximum power point] have in many cases remained matters of conjecture - the nature and presence of trap-assisted recombination are frequently deduced from ideality factor measurements or electrical transient methods, such as transient photovoltage, performed under opencircuit conditions. However, these methods have been shown to be susceptible to both electrode-induced and resistive/transport-related limitations. [48, 162, 168, 178, 194] To date, reliable methods that unambiguously quantify photocurrent losses caused by trap-assisted recombination in organic solar cells under relevant operating conditions are lacking.

Herein, wide dynamic range light-intensity-dependent measurements of the external quantum efficiency [EQE] at sub-Hz modulation frequencies are utilized to characterize trap-assisted recombination in fully operational organic photovoltaic devices [see Box 5.1]. It is shown that in the presence of traps, an anomalous and hitherto unreported two-step EQE behaviour can be observed [as a function incident light intensity]. The two-step behaviour is a results of trap-included first-order recombination in the bulk [which is absent at low intensities] being switched on at moderate intensities due to trap filling. This effect allows for the first-order trap-assisted recombination losses under 1 sun intensity to be quantified. Combined intensity-dependent photocurrent $[\mathrm{IPC}]$ and open-circuit voltage $\left[V_{\mathrm{oc}}\right]$ measurements further allow an estimate of the energy and density of trap states within the active layer. Using this method, trap-induced first-order photocurrent losses are found to be present in a large variety of fullerene and non-fullerene-based organic BHJ solar cells - a sufficient enough set of material systems to consider the observation universal. This first-order recombination loss is caused by deep traps states in the gap, lying in all cases $0.35-0.6 \mathrm{eV}$ below the transport levels. Finally, the findings are also relevant for indoor solar harvesting and photodetector applications of organic BHJs operating at low intensities. 


\subsection{Shockley-Read-Hall statistics: shallow and deep trap mode}

In Fig. 6.1 the basic principles behind the analysis method is demonstrated. The inset in Fig. 6.1 a shows a schematic energy level diagram for a BHJ with electron trap states of density $N_{\mathrm{t}}$ situated at an energy $E_{\mathrm{t}}$ in the gap. The trap depth is then defined as $\Delta_{\mathrm{t}}=E_{\mathrm{L}, \mathrm{A}}-E_{\mathrm{t}}$, where $E_{\mathrm{L}, \mathrm{A}}$ is the LUMO level energy of the acceptor.

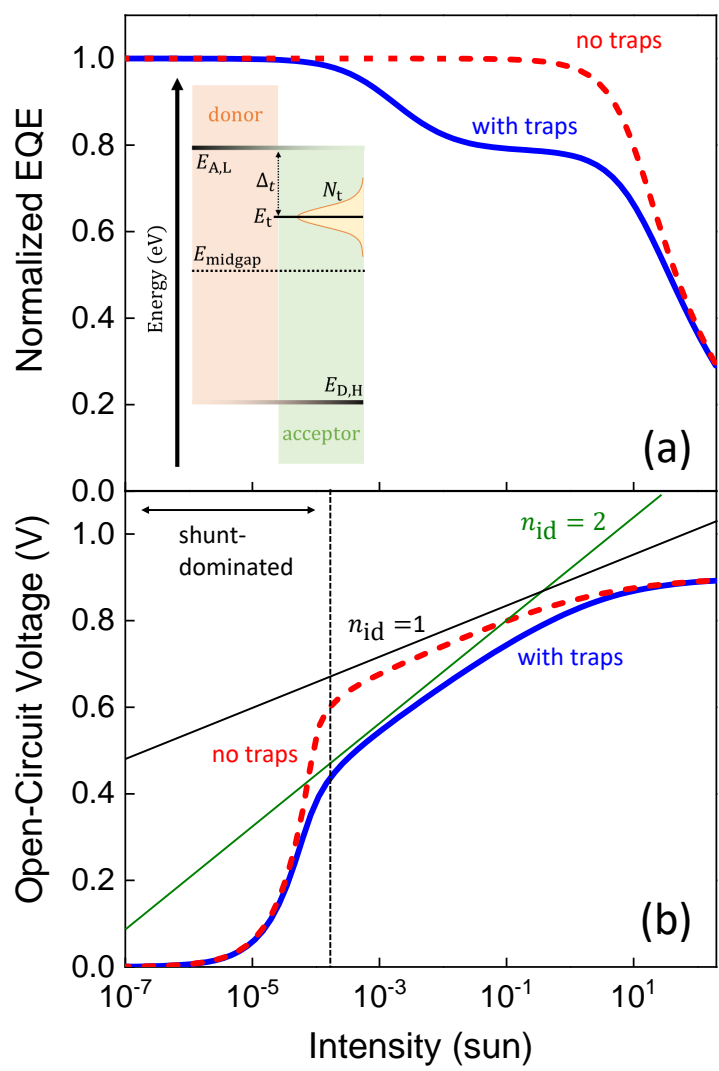

Figure 6.1: Comparison of simulated external quantum efficiency [EQE] (a) and open-circuit voltage (b) versus intensity between the case with [blue, solid line] and without [red, dashed line] trap states in the donor: acceptor blend bulk. The inset in (a) shows the schematic energy level diagram of donor: acceptor BHJ solar cell with acceptor LUMO level $\left[E_{\mathrm{A}, \mathrm{L}}\right]$ and donor HOMO level $\left[E_{\mathrm{D}, \mathrm{H}}\right]$. The electron trap is located at an energy $\left[E_{\mathrm{t}}\right]$ having a trap depth of $\Delta_{\mathrm{t}}=\left|E_{\mathrm{A}, \mathrm{L}}-E_{\mathrm{t}}\right|$ and a trap density of $N_{\mathrm{t}}$.
In accordance with the Shockley-ReadHall [SRH] formalism [see Chapter $\mathbf{1}$, Section 1.8.2, the associated trapassisted recombination takes place between trapped electrons [captured from the LUMO] and free holes. Fig. 6.1 b,c show the simulated normalized EQE and $V_{\text {oc }}$ versus light intensity $\left[I_{\mathrm{L}}\right]$ for a generic organic solar cell with [blue solid line] and without [red dashed line] traps. A numerical drift-diffusion model was used for the simulations 80 , 81]; the details of which are provided in the Tab. A.3 in the Appendix A. For the device with traps, the simulations reveal a constant EQE at low intensities and a second semi-plateau at higher intensities [albeit with lower magnitude] caused by the first-order trap-assisted recombination in the bulk. For the trap-free device, the second plateau is notably absent.

The SRH rate is limited by the number of trapped electrons, which depends on the position of the quasi-Fermi level $E_{\mathrm{F}, \mathrm{n}}$ for free electrons relative to the 
trap energy $E_{\mathrm{t}}$. The carrier density, and thus the quasi-Fermi level, in turn, depend on the incident light intensity. As discussed in detail in Chapter 5, Section 5.6, under conditions when $E_{\mathrm{F}, \mathrm{n}} \ll E_{\mathrm{t}}$ [low $I_{\mathrm{L}}$ ], most of the traps are unoccupied [shallow trap mode], and the trap-assisted recombination is negligible [compared to charge extraction]. In contrast, when $E_{\mathrm{F}, \mathrm{n}} \gg E_{\mathrm{t}}$ [high $I_{\mathrm{L}}$ ], a significant fraction of traps will be occupied by electrons [deep trap mode], and first-order trap-assisted recombination is switched on, resulting in the second EQE plateau. The critical electron density at which $E_{\mathrm{F}, \mathrm{n}}=E_{\mathrm{t}}$ is given by

$$
n_{1}=N_{\mathrm{L}, \mathrm{A}} \times \exp \left[-\frac{\Delta_{\mathrm{t}}}{k_{\mathrm{B}} T}\right]
$$

where $N_{\mathrm{L}, \mathrm{A}}$ is the density of transport states in the LUMO of the acceptor, $k_{\mathrm{B}}$ is the Boltzmann constant and $T$ is the absolute temperature. Hence, the trap depth critically defines the onset intensity for first-order SRH recombination in the bulk- below this onset, the traps are acting as shallow traps and trap-induced recombination losses in the bulk are small. Note that at higher intensities second-order bimolecular recombination eventually starts to play a role, manifest as an additional light-intensity dependent quantum efficiency $[\mathrm{QE}]$ loss.

It should be noted that the competition between trap-assisted recombination and bimolecular recombination is more pronounced under open-circuit conditions. The corresponding ideality factor $n_{\text {id }}$ is related to the open-circuit voltage via $q V_{\text {oc }} \approx$ $n_{\text {id }} k_{\mathrm{B}} T \ln \left(I_{\mathrm{L}}\right)$, where $q$ is the elementary charge. As evident from Fig. 6.1 b, while the $\mathrm{V}_{\text {oc }}$ at low and high intensity is limited by shunt effects $\left[n_{\text {id }}>2\right]$ and the contacts $\left[n_{\text {id }}<1\right]$, respectively, the $V_{\text {oc }}$ at moderate intensities is dominated by bulk recombination. Depending on the relative balance between the first-order trap-assisted recombination $\left[n_{\mathrm{id}}=2\right]$ and bimolecular recombination $\left[n_{\mathrm{id}}=1\right]$, the ideality factor transition from 1 to 2 resulting in arbitrary values between 1 and 2 when varying the photovoltage [through control of the incident light intensity]. 


\section{DIRECT OBSERVATION OF TRAP-ASSISTED RECOMBINATION}

\subsection{Universal presence of trap states}

In the following, intensity-dependent EQE measurements are used to experimentally quantify the QE loss caused by trap-assisted recombination in the bulk [see Box 5.1]. For this purpose, IPC measurements under short-circuit conditions at an excitation wavelength of $520 \mathrm{~nm}$ were conducted. The associated two-step EQE behaviour is distinctly different to that observed for other first-order loss mechanisms [e.g., of CT states and excitons] which are expected to be independent of light intensity [see Chapter 5. Thus, the presence of such two-step EQE can be used to unambiguously identify trapassisted recombination in organic photovoltaic devices. Note that to experimentally detect first-order recombination via trap states, the photocurrent needs to be recorded over a broad intensity range and at extremely low modulation frequencies $[f]$ so that $1 / f$ is longer than the trap release time. Due to the dominant impact of flicker noise at low frequencies, such measurements typically require long integration times [here more than 30s for each intensity point at low intensity].

Fig. 6.2 a,b show the light intensity dependent normalized EQE and $V_{\mathrm{oc}}$, respectively, of three different organic solar cells: PCDTBT:PC ${ }_{70} \mathrm{BM}, \mathrm{PTB} 7-\mathrm{Th}: \mathrm{PC}_{70} \mathrm{BM}$ and PM6:BTP-eC9. The chemical definitions are provided in the Appendix A. All devices exhibit a constant EQE plateau at low irradiances reaching a second semi-plateau at moderate irradiance with lower magnitude, consistent with the presence of trap-assisted recombination in the bulk as predicted by the simulations [see Section 5.6 in Chapter 5, and Fig. B.6 in Appendix B. Corresponding first-order, trap-induced, relative QE losses of $5 \%, 4 \%$ and $3 \%$ were obtained from the IPC measurements for the three systems, respectively. However, this loss channel is deactivated below intensities around $10^{-3}-10^{-4}$ suns for PCDTBT:PC $70 \mathrm{BM}$ and PBT7-Th:PC $70 \mathrm{BM}$, and $10^{-2}$ suns for PM6:BTP-eC9. The presence of trap-assisted recombination is further corroborated by the $V_{\text {oc }}$ data with ideality factors varying between 1 and 2 at moderate intensities. At intensities above 1-sun equivalent, higher-order processes [such as bimolecular recombination and series resistance limitations] eventually lead to a rapid decrease of the EQE. 
(a)

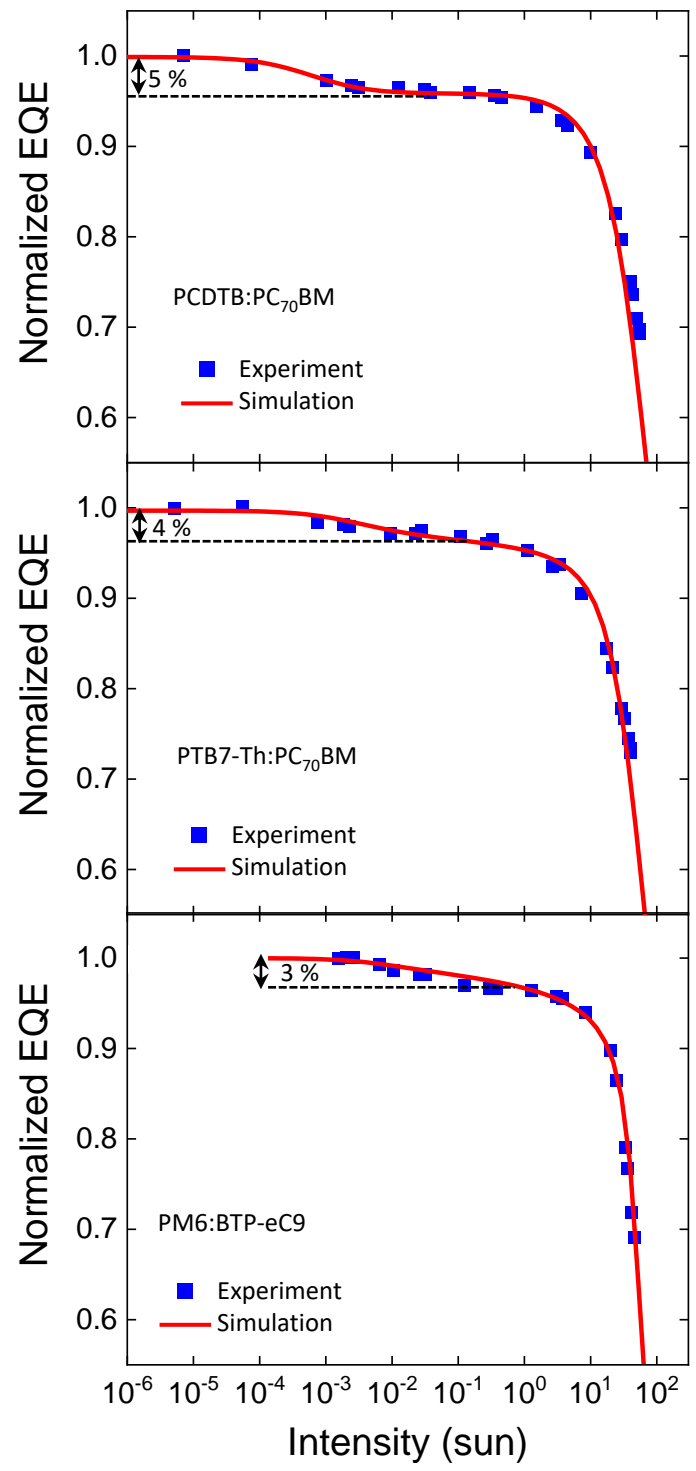

(b)

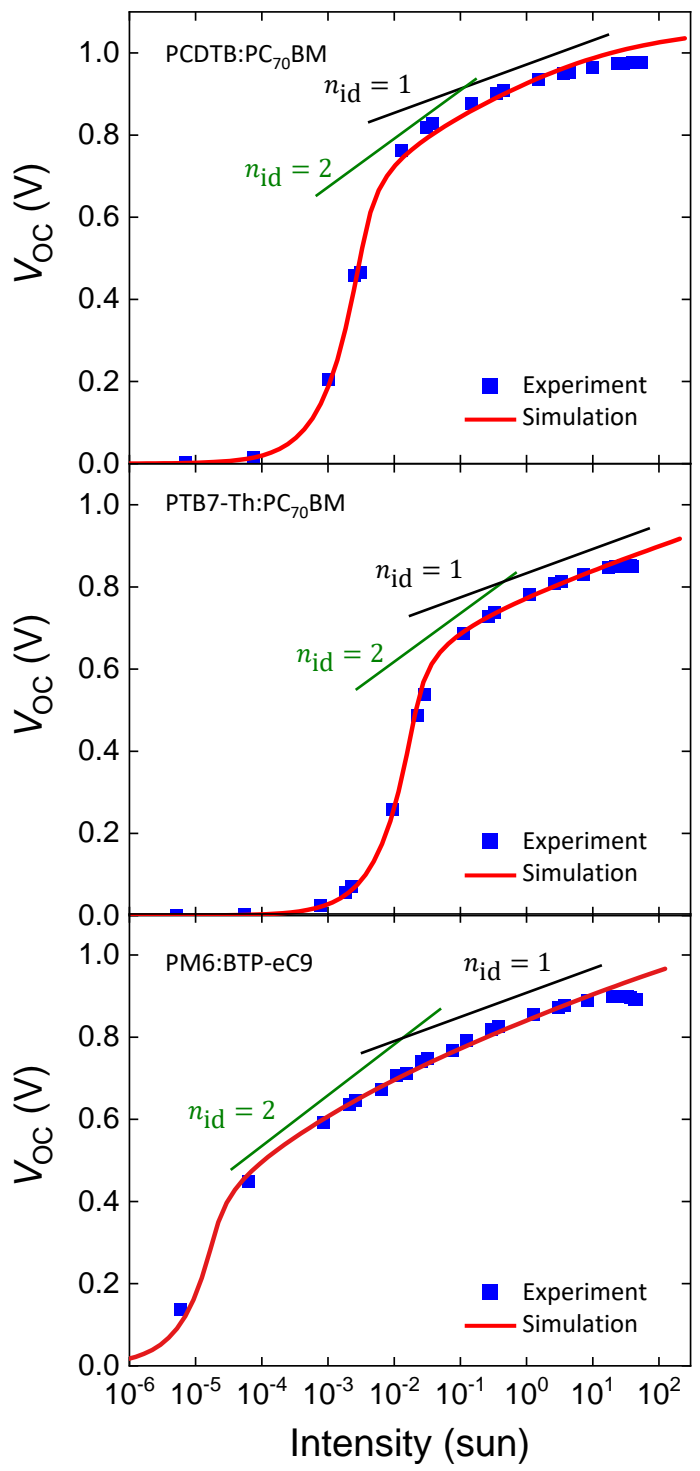

Figure 6.2: a) Experimental [blue symbols] and simulated [red lines] normalized external quantum efficiency [EQE] plotted as a function of intensity for three different organic solar cells together with the estimated relative quantum efficiency [QE] losses induced by first-order, trap-assisted recombination: PCDTBT:PC ${ }_{70} \mathrm{BM}[5 \%]$, PTB7-Th:PC ${ }_{70} \mathrm{BM}$ [4 \%] and PM6:BTP-eC9 [3\%]. b) Corresponding open-circuit voltage $\left[V_{\text {oc }}\right]$ plotted as a function of intensity for the three solar cells. The green and black lines are guides to the eye with a slope corresponding to an ideality factor of $n_{\mathrm{id}}=2$ [when trap-assisted recombination is dominant] and $n_{\mathrm{id}}=1$ [in the case bimolecular recombination dominates]. The experimentally obtained and simulated $J-V$ curves are provided in Fig. B.7 in Appendix B. 


\section{DIRECT OBSERVATION OF TRAP-ASSISTED RECOMBINATION}

This two-step EQE behavior was detected for a large variety of fullerene and non-fullerene acceptor based organic solar cells including state-of-the-art PM6:BTPeC9. Current density versus applied voltage $[J-V]$ performances under artificial AM $1.5 \mathrm{G}$ conditions together with the intensity dependent, normalized EQEs are shown in Fig. 6.3a,b. The PCEs of the studied systems are summarized in Fig. 6.4a. The associated relative $\mathrm{QE}$ losses for the studied systems are summarized in Fig. 6.4d. Note that the relative QE losses are not only an indication of how much [relative] photocurrent is lost due to the first-order, trap-assisted recombination channels, but also how much [relative] more photocurrent could be gained in [theoretical] absence of SRH recombination pathways. The corresponding slope parameter $\alpha$ of the short-circuit density, $J_{\mathrm{sc}} \sim I_{\mathrm{L}}^{\alpha}$, in the intensity regime of the second EQE semi-plateau is shown in Fig. 6.4 e. For all systems, an $\alpha$ close to unity was obtained, indicating that the QE loss associated with the second plateau is first-order. Although every possible combination of acceptor and donor organic semiconductor cannot realistically studied, the consistency of these findings point toward the universal presence of first-order recombination via deep traps in the bulk of organic photovoltaic junctions. To estimate the associated trap depth, Eq. 6.1 [see Section 5.6 in Chapter 5] is used. Accordingly, the trap depth can be obtained from the free carrier density $\left[n=n_{1}\right]$ at the point-of-transition [POT] intensity right in-between the two EQE plateaus. The photogeneration current at POT is given by $J_{\mathrm{POT}}=2 q n d / t_{\mathrm{tr}}$. Hence, the trap depth can be estimated via

$$
\Delta_{\mathrm{t}}=k_{\mathrm{B}} T \ln \left[\frac{2 q N_{\mathrm{L}, \mathrm{A}} d}{J_{\mathrm{POT}} t_{\mathrm{tr}}}\right] .
$$




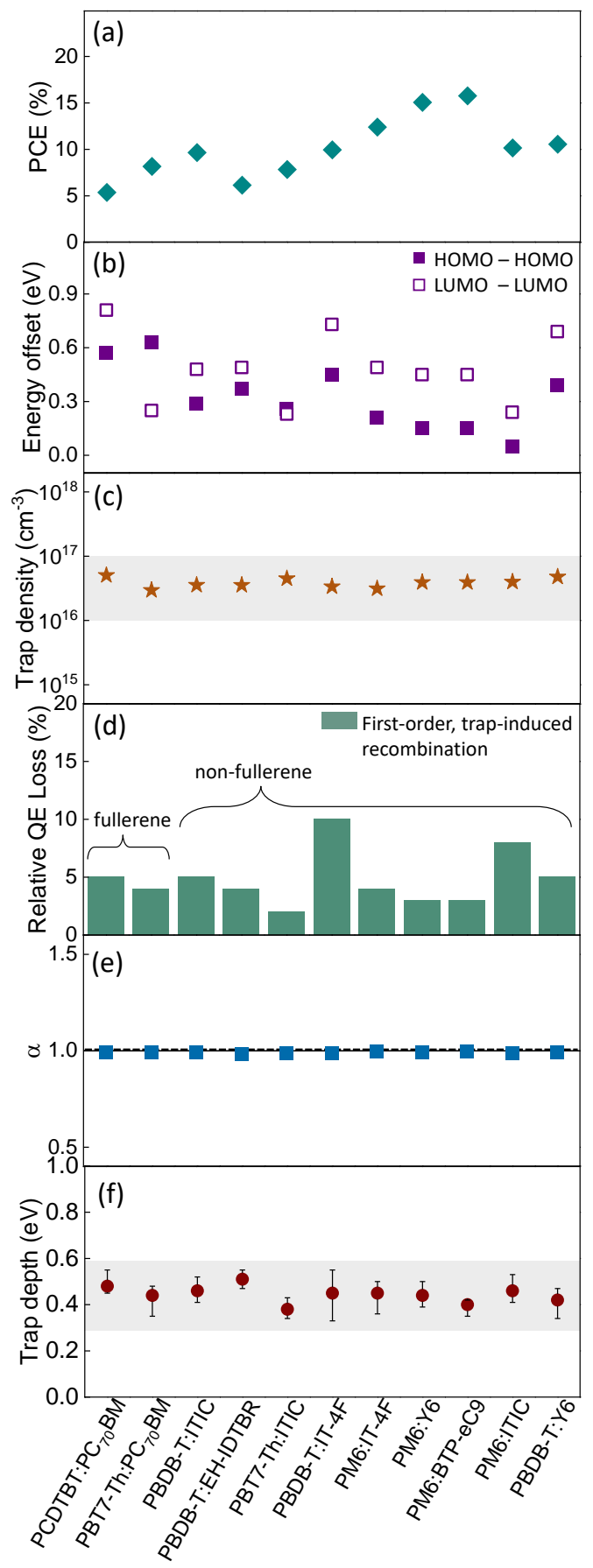

Figure 6.4: (a) Power conversion efficiency [PCE], (b) energetic offset, (c) trap density, (d) relative quantum efficiency [QE] loss, (e) slope parameter $\alpha$, and (f) trap depth of organic solar cells.
Fig. 6.4f shows the trap depths for the different systems. The transit-times were obtained from resistance-dependent photovoltage [see Box 3.3 measurements [see Fig. B.7 in Appendix B. The trap depths are between $0.35-0.6 \mathrm{eV}$ for all systems [see Fig. $6.4 \mathbf{f}$ ]. The extracted trap depths are consistent with those experimentally expected from the measured open-circuit voltages [see Fig. B.8 in Appendix B. The experimental EQE and $V_{\text {oc }}$ [and $J-V$ curves; see Fig. B.7 in Appendix B can be reproduced qualitatively by DD simulations, assuming a device with trap states lying $0.4-0.5$ $\mathrm{eV}$ below the transport levels and a finite shunt resistance, as indicated by the solid red lines in Fig. 6.2 a,b. Subsequently, and considering the same set of traps, the intensity-dependent features of both EQE and $\mathrm{V}_{\mathrm{oc}}$ can be consistently explained. In particular, the presence of deep traps can account for the transitioning ideality factors between 1 and 2 at moderate intensities [see Fig. 6.1]. In fact, these results provide a possible and very plausible explanation for the origin of ideality factors in organic solar cells. An estimate of the trap density $N_{\mathrm{t}}$ can be obtained by making use of Eq. $\mathbf{5 . 1 9}$ [see Section 5.6.3 in Chapter 5. Based on this estimate, $N_{\mathrm{t}}$ is found to be on the order of $10^{16}-10^{17} \mathrm{~cm}^{-3}$ [see Fig. 6.4c]. 


\section{DIRECT OBSERVATION OF TRAP-ASSISTED RECOMBINATION}

\section{Critical trap depth and implications for photodetectors}

\section{Box 6.1}

The results from Fig. 6.4 are consistent with the presence of trap-assisted recombination that is activated at moderate intensities by trap filling of states, lying $0.35-0.6 \mathrm{eV}$ below the transport levels, acting as deep trap states under 1 sun illumination. The onset intensity, at which the first-order trap-assisted recombination in the bulk is activated, is determined by the trap depth [see Eq. 6.2. Concomitantly, the so-called critical trap depth, $\Delta_{\mathrm{t}}^{*}$, below which these trap-induced losses may be avoided $\left[\Delta_{\mathrm{t}}<\Delta_{\mathrm{t}}^{*}\right]$, is given by

$$
\Delta_{\mathrm{t}}^{*} \approx k_{\mathrm{B}} T \ln \left[\frac{2 q N_{\mathrm{L}, \mathrm{A}} d}{J_{\mathrm{ph}} t_{\mathrm{tr}}}\right],
$$

where $J_{\mathrm{ph}}$ is the corresponding photocurrent density. Assuming typical values of $t_{\mathrm{tr}} \sim 1 \mu \mathrm{s}, N_{\mathrm{L}, \mathrm{A}} \sim 10^{20} \mathrm{~cm}^{-3}$, and $d=100 \mathrm{~nm}$ to avoid trap-induced losses in organic solar cells the trap depth needs to be smaller than $0.25 \mathrm{eV}$. However, $\Delta_{\mathrm{t}}^{*}$ only needs be smaller than $0.4 \mathrm{eV}$ in indoor solar cells [operating usually at $\sim 0.3$ $\%$ of 1 sun]. This suggests that the associated photocurrent losses in indoor light harvesting solar cells, and similar applications such as photodetectors, operating at low light intensities, could be avoided. 


\subsection{Photoexcitation pathways of trap states}

The presence of trap states in these systems is also visible from ultra-sensitive EQE measurements, performed using an approach described in detail in Chapter 2 which allows to probe sub-gap features far below the charge-transfer [CT] state energy. [82] This is demonstrated for the model system PCDTBT:PC ${ }_{70} \mathrm{BM}$ in Fig. 6.5. To confirm that these features are associated with first-order trap-assisted recombination, trace amounts of m-MTDATA were intentionally added to the PCDTBT:PC $\mathrm{P}_{70} \mathrm{BM}$ active layer. Here, m-MTDATA will specifically act as a hole trap due to its HOMO energy level of $5.1 \mathrm{eV} \cdot[100$
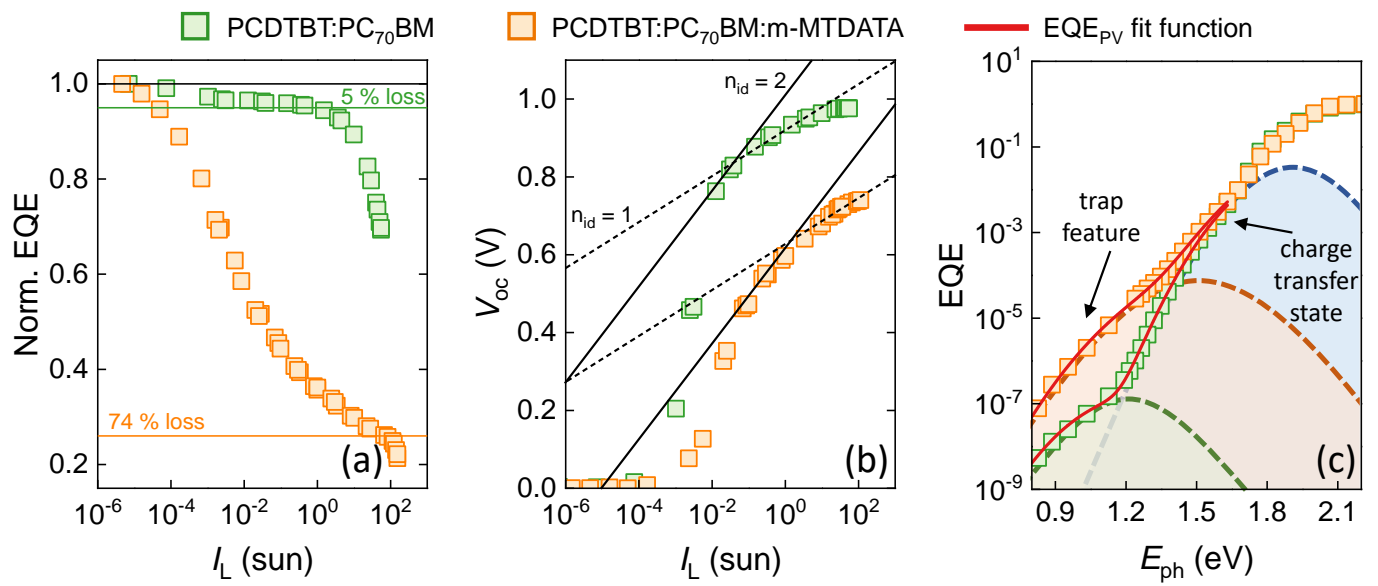

Figure 6.5: (a) Light intensity dependent, normalized external quantum efficiency [EQE] of a PCDTBT:PC ${ }_{70} \mathrm{BM}$ solar cell plotted as a function of light intensity $\left[I_{\mathrm{L}}\right]$, and compared to a PCDTBT:PC 70 BM:m-MTDATA [ $1 \%$ by mole] device. $\mathbf{b}$ ) Open-circuit voltage $\left[V_{\mathrm{oc}}\right]$ of a PCDTBT:PC ${ }_{70} \mathrm{BM}$ and PCDTBT:PC ${ }_{70} \mathrm{BM}: \mathrm{m}-\mathrm{MTDATA}$ [ $1 \%$ by mole] solar cell plotted as a function of light intensity. Dotted and solid lines are guides to the eye corresponding to an ideality factor of $n_{\text {id }}=1$ and $n_{\text {id }}=2$. c) EQE spectra of PCDTBT:PC ${ }_{70} \mathrm{BM}$ and PCDTBT:PC ${ }_{70}$ BM:m-MTDATA [1 \% by mole] BHJ solar cells plotted as a function of photon energy $\left[E_{\mathrm{ph}}\right]$. EQEs at photon energies below the gap are fitted with a double Marcus function [red solid lines] accounting for both charge transfer state [blue shaded area] and sub-gap absorption features [green and orange shaded areas].

As evident from Fig. 6.5 a,b, the degree of trap-assisted recombination and associated $\mathrm{QE}$ loss in PCDTBT: $\mathrm{PC}_{70} \mathrm{BM}$ is drastically increased as one would have expected by adding $1 \%$ [by mole] m-MTDATA with respect to PCDTBT weight fraction, corresponding to approximately $0.9 \%$ molar ratio. The associated trap depth was estimated to be $0.37 \mathrm{eV}$, corresponding to a hole trap energy of $E_{\mathrm{t}} \approx 4.9 \mathrm{eV}$ [assum- 


\section{DIRECT OBSERVATION OF TRAP-ASSISTED RECOMBINATION}

ing $E_{\mathrm{HOMO}}=5.3 \mathrm{eV}$ for PCDTBT[195], close to the HOMO level of m-MTDATA. Moreover, the trap density is estimated to increase from $N_{\mathrm{t} \text {,neat }} \approx 5 \times 10^{16} \mathrm{~cm}^{-3}$ in neat PCDTBT:PC $70 \mathrm{BM}$ to $N_{\mathrm{t}, \text { added }} \approx 10^{18} \mathrm{~cm}^{-3}$ after adding m-MTDATA. Although this is only a rough estimate, the number density of added traps, relative to the total density of available transport sites, is consistent with the added $1 \%$ m-MTDATA by mole. The corresponding ultra-sensitive EQE versus incident light photon energy is shown in Fig. 6.5c. Assuming Marcus-type charge-transfer, the low-energy sub-gap feature is expected to correspond to the excitation of a chargetransfer complex between a free and a trapped charge carrier, as recently shown by Zarrabi and co-workers. [49] The corresponding CT energy of the sub-gap feature in the neat PCDTBT: $\mathrm{PC}_{70} \mathrm{BM}$ is estimated to be $E_{\mathrm{CT} \text {,trap }} \approx 0.74 \mathrm{eV}$. [49] With the trap depth $\Delta_{\mathrm{t}}=0.48 \mathrm{eV}$, and assuming a donor: acceptor effective gap of $E_{\mathrm{g}} \approx 1.4 \mathrm{eV}$ for charge-separated states, this corresponds to a binding energy $\left[E_{\mathrm{b}}=E_{\mathrm{g}}-\Delta_{\mathrm{t}}-E_{\mathrm{CT}, \text { trap }}\right]$ of 0.18 $\mathrm{eV}$. With the addition of m-MTDATA the sub-gap feature is blue-shifted, consistent with the smaller trap depth $\left[E_{\mathrm{CT}, \text { trap }} \sim\right.$ $0.85 \mathrm{eV}]$. Furthermore, the trap feature is seen to increase [see Box 6.2], which agrees well with the estimated increase in trap density [see above]. It is a subtle but important point to note that both devices had the same active layer thickness in order to reduce the influence of the optical interference effects on the sub-gap EQE. [42] These findings suggest that the low-energy sub-gap features observed in

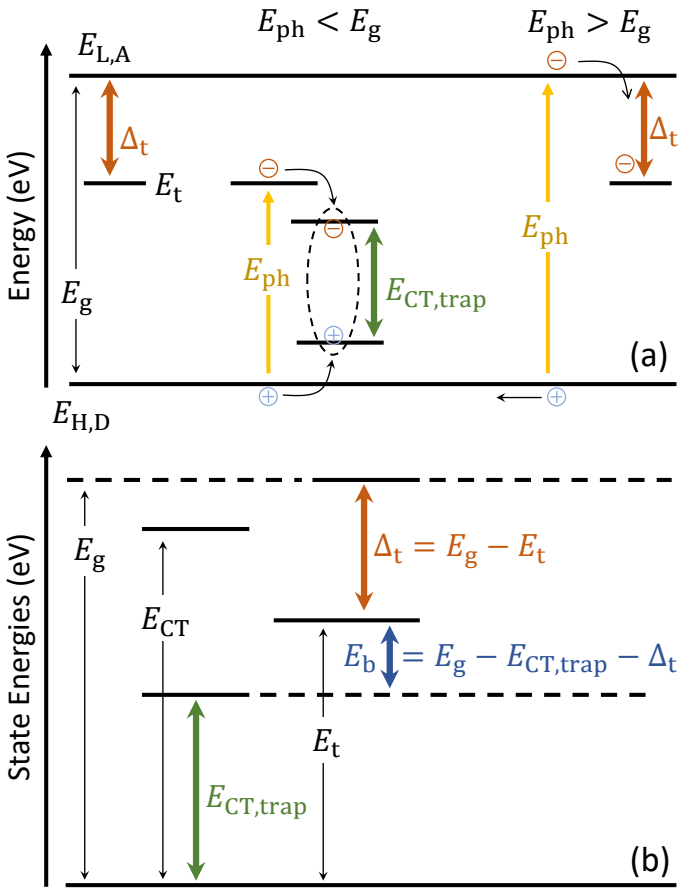

Figure 6.6: (a) Schematic energy level diagram of a donor: acceptor blend with $E_{\mathrm{L}, \mathrm{A}}$ and $E_{\mathrm{H}, \mathrm{D}}$ denoting the acceptor LUMO and donor HOMO energy level. The [electron] trap state is located at an energy $E_{\mathrm{t}}$ within the donor: acceptor gap $E_{\mathrm{g}}$. The associated charge generation paths in ultrasensitive EQE [excitation energy $E_{\mathrm{ph}}$ smaller than $\left.E_{\mathrm{g}}\right]$ and IPC $\left[E_{\mathrm{ph}}\right.$ larger than $\left.E_{\mathrm{g}}\right]$ measurements are indicated by up-and-downward arrows. (b) State energies and trap-related parameters in organic solar cells, which are obtained as such from ultra-sensitive EQE $\left[E_{\mathrm{CT}, \text { trap }}\right]$ and IPC measurements $\left[\Delta_{\mathrm{t}}\right]$, and are interconnected via the binding energy $E_{\mathrm{b}}$. 
the ultra-sensitive EQE measurements are of the same origin as the deep trap states probed by IPC, with the difference being alternate photoexcitation paths. While in ultra-sensitive EQE measurements the trap states are directly excited [i.e., the photon excitation energy $E_{\mathrm{ph}}$ is much lower than $E_{\mathrm{g}}$ ] forming Coulombically bound trappedelectron/mobile-hole pairs in case of electron traps, in IPC measurements the trapping of free, separated, charge carries with excess energy [since $E_{\mathrm{ph}}>E_{\mathrm{g}}$ ] is observed [see Fig. 6.6a]. The two parameters, trap depth $\Delta_{\mathrm{t}}$ [obtained from IPC] and chargetransfer trap energy $E_{\mathrm{CT} \text {,trap }}$ [obtained from ultra-sensitive EQE measurements], are related via a binding energy $E_{\mathrm{b}}=E_{\mathrm{g}}-\Delta_{\mathrm{t}}-E_{\mathrm{CT} \text {,trap }}$, which generally leads to a reduction of the trap depth for separated charge carriers [see Fig. 6.6b].

\section{Details of sub-bandgap $\mathrm{EQE}_{\mathrm{PV}}$ fit functions}

\section{Box 6.2}

A double-Gaussian function is used to fit the reduced EQE [i.e., EQE times photon energy; $\left.\mathrm{EQE}_{\mathrm{red}}\right]$ at photon energies below the gap accounting for both charge transfer state [CTS] and trap state absorption features. The associated fit function is given by 75

$$
\begin{aligned}
\mathrm{EQE}_{\mathrm{red}}(h \nu) & =\frac{A_{\mathrm{CTS}}}{\sigma_{\mathrm{CTS}} \sqrt{2 \pi}} \times \exp \left[-\frac{\left(h \nu-E_{\mathrm{CTS}}\right)^{2}}{2 \sigma_{\mathrm{CTS}}^{2}}\right] \\
& +\frac{A_{\text {trap }}}{\sigma_{\text {trap }} \sqrt{2 \pi}} \times \exp \left[-\frac{\left(h \nu-E_{\text {trap }}\right)^{2}}{2 \sigma_{\text {trap }}^{2}}\right],
\end{aligned}
$$

where $h \nu$ is the photon energy, $\sigma$ is related to the width [which depends on the reorganization energy], $A$ denotes the area of the fit function [which is related to the number density of states], while $E_{\mathrm{CT}}$ and $E_{\text {trap }}$ denote the fit function peak positions. 


\section{DIRECT OBSERVATION OF TRAP-ASSISTED RECOMBINATION}

\subsection{Trap states: electron acceptor- or donor-type?}

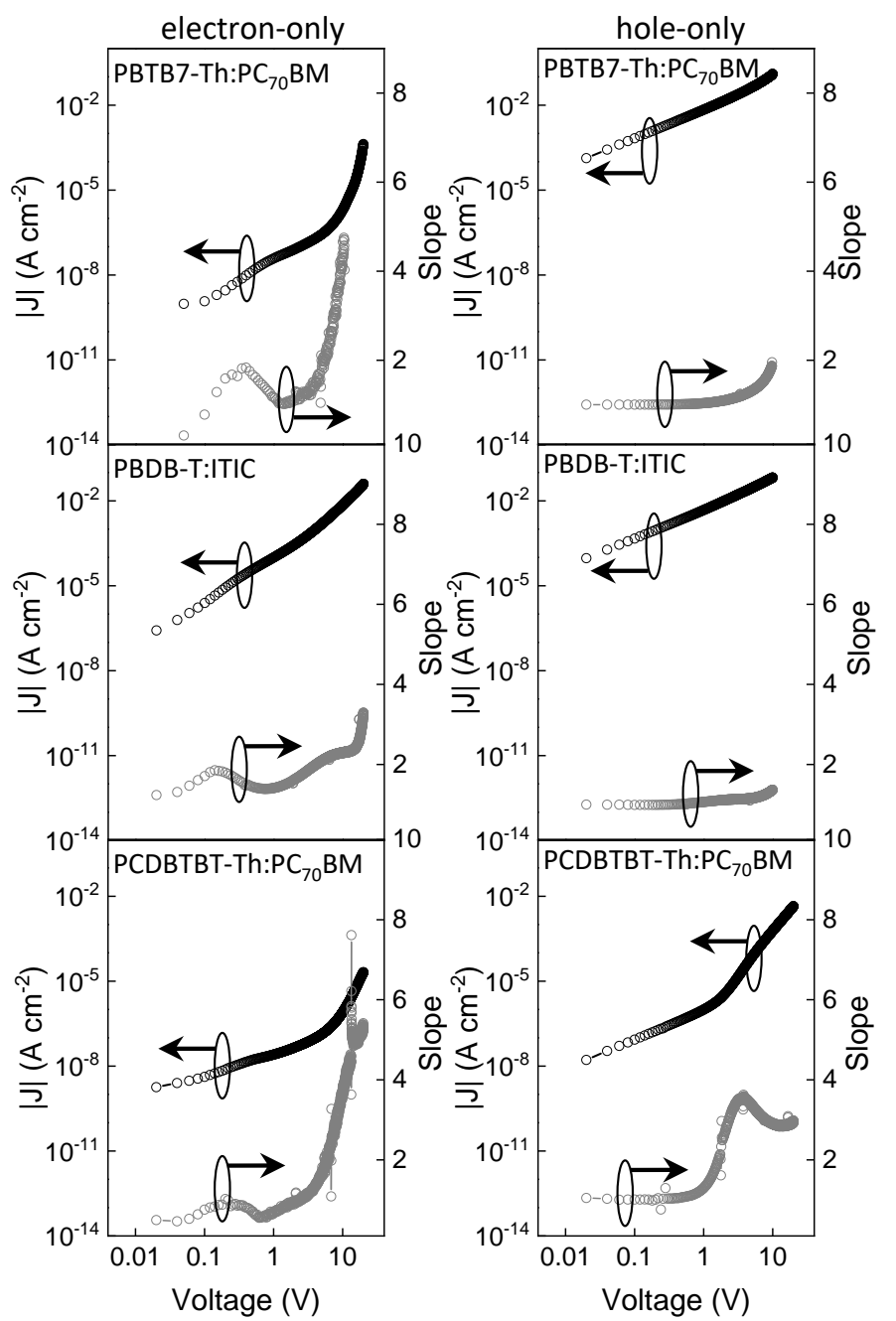

Figure 6.7: Dark current density $|J|$ [left axis] and calculated slope [right axis] versus applied voltage of a 1400 nm thick PTB7-Th:PC 70 BM, 800 nm thick PBDB-T:ITIC and $1100 \mathrm{~nm}$ thick PCDTBT:PC ${ }_{70} \mathrm{BM}$ electron-only [left column] and hole-only [right column] device. Details of the SCLC technique are provided in Box 6.3.
It should be noted that the exact value of $E_{\mathrm{b}}$ is expected to be strongly influenced by the prevailing energetic disorder. [42] From the above analysis it is unclear whether the observed traps are electron or hole traps. For this purpose, dark space-chargelimited current [SCLC] measurements on electron-only and hole-only devices were conducted [see Fig. 6.7]. Information to [single-carrier] device fabrication are provided in Appendix A; experimental details to dark SCLC measurement technique can be found in Box 6.3. The SCLC results point towards these traps being predominately acceptor-type electron traps. As such, weak humps in the slope of the PTB7-Th:PC 70 BM and the PBDB-T:ITIC electron-only [PCDTBT:PC 70 BM hole-only] device reveal electron [hole]

trap states [180, 196]). However, it should be stressed that SCLC is very challenging being highly sensitive to energetics at the contacts, [unintentional] doping in the bulk, and energetic disorder, complicating the analysis of the experimental data. 196 198] 


\section{Experimental details of space-charge-limited-current (SCLC) measurements}

\section{Box 6.3}

Space-charge-limited-current [SCLC] measurements are performed using a highperformance, low noise source-meter-unit [Keithley 2450] to apply bias voltage and measure the device under test [DUT] dark current density. For the SCLC measurements, the DUT is mounted in an electrically shielded, low noise Linkam sample holder to suppress hum noise and measure the current density sensitively.

For a trap-free [trap-limited] device, the dark current density $J$ follows

$$
J=\frac{4 \pi^{2} \varepsilon \varepsilon_{0} k_{\mathrm{B}} T \mu}{q d^{3}} V\left[\times \frac{N_{\mathrm{C}}}{N_{\mathrm{t}}} \exp \left\{-\frac{\Delta_{\mathrm{t}}}{k_{\mathrm{B}} T}\right\}\right]
$$

at small voltages [ohmic diffusion (trap-controlled) limit]. At high voltage, in turn, the dark current density can be expressed as

$$
J=\frac{9 \varepsilon \varepsilon_{0} \mu}{8 d^{3}} V^{2}
$$

for both trap-limited and trap-free cases. The trap density $N_{\mathrm{t}}$ can be obtained at the voltage $V_{\mathrm{TFL}}$, at which the trap-filling limit is reached:

$$
V_{\mathrm{TFL}}^{*} \sim \frac{q N_{\mathrm{t}} d^{2}}{2 \varepsilon \varepsilon_{0}} .
$$

In this regard it should be also noted that the traps, and the associated losses, seem to be agnostic to changes in morphology [see Box 6.4. The observed trap depths are, however, consistent with the findings by Zuo et al. In the work by Zuo et al., it was proposed that electron [and hole] traps lying $\sim 0.3-0.4 \mathrm{eV}$ below [or above] the LUMO [HOMO] are induced by dielectric effects from water-filled nanovoids in neat organic semiconductor films. 180] A similar situation is also expected to occur for BHJ structures, pointing towards a universal presence of water-filled nanovoids in BHJs. A detailed morphological structure-property analysis is, however, needed to further confirm this proposition. 


\section{Active layer treatment of PTB7-Th:PC $70 \mathrm{BM}$}

\section{Box 6.4}

It has been reported that $\mathrm{PTB} 7-\mathrm{Th}: \mathrm{PC}_{70} \mathrm{BM}$ solar cells processed with $\mathrm{CB}$ solution or CB:DIO solution could render contrasting morphology and defects in active layer. Hence, PTB7-Th:PC ${ }_{70} \mathrm{BM}$ devices with and without DIO were fabricated [details to device fabrication are provided in Appendix $\mathbf{A}$ ].
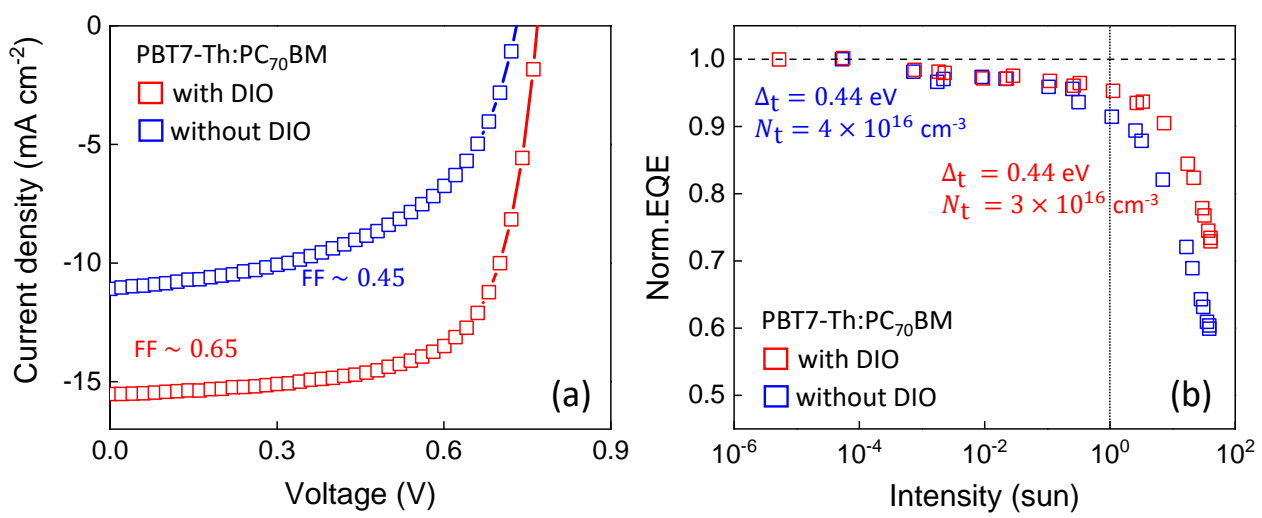

Figure 6.8: (a) Light $J$ - $V$ curves and (b) light-intensity dependent, normalized EQE of PBT7-Th:PC ${ }_{70}$ BM fabricated with [red] and without [blue] DIO.

The corresponding current density versus applied voltage $[J-V]$ characteristics under artificial 1 sun AM 1.5G condition [see Box 3.1], and normalized EQE, based on intensity dependent photocurrent [IPC] measurements [see Box 5.1], are shown in Fig. 6.8. It has been suggested that the different processing methods are linked to different morphology in the active layer.[60, 199-201] Based on IPC, an increased second-order recombination in $\mathrm{PTB} 7-\mathrm{Th}: \mathrm{PC}_{70} \mathrm{BM}$ processed without DIO [blue symbols in Fig. 6.8 b] can be discerned, consistent with the drastic reduction in the fill factor [see Fig. 6.8 a; photovoltaic parameters are provided in Tab. A.2 in Appendix B. Nevertheless, the differences in the trap depth and trap density for PTB7-Th:PC ${ }_{70} \mathrm{BM}$ with and without DIO were found to be negligible [see inset in Fig. 6.8 b] pointing towards a trap origin that is not directly linked to morphology. 


\subsection{Conclusion}

In this chapter it has been shown that first-order recombination losses caused by traps are universally present in a large variety of fullerene and non-fullerene acceptor-based organic semiconductor BHJ solar cells. This loss is caused by deep trap states situated $\sim 0.35-0.6 \mathrm{eV}$ below the transport edges of the acceptor: donor blend having trap densities between $10^{16}-10^{17} \mathrm{~cm}^{-3}$. The associated trap-assisted recombination not only induces losses in the photocurrent, but also limit the open-circuit voltage giving rise to ideality factors generally between 1 and 2 . These findings not only shed new light on the nature, dynamics, and role of traps in light harvesting organic semiconductor devices, but also reveal new insight into the measurement, interpretational complexities, and variability of ideality factors in solar cells. Wide dynamic range, modulated intensitydependent measurements are a powerful tool in probing the fundamental structureproperty relationships in photovoltaic materials. 
6. DIRECT OBSERVATION OF TRAP-ASSISTED RECOMBINATION 


\section{Chapter 7}

\section{Summary and Outlook}




\section{SUMMARY AND OUTLOOK}

With recent power conversion efficiencies exceeding $18 \%$, and $20 \%$ on the horizon, next-generation optoelectronic devices comprised of organic semiconducting material blends are an attractive alternative to their inorganic counterparts and show substantial promise for solar cells, photodetectors, and light-emitting diode applications. To push the boundaries of efficiency even further, and to make laboratory-small-area organic photovoltaic competitive for lab-to-fab scaling in industrial markets, further knowledge and progress in molecule synthesis, device engineering, and electro-optical characterization are required. This requires an in-depth analysis of fundamental processes governing basic device physics allowing stringent optimization of processing, clear definition of material requirements, and the realization of new organic semiconductors to meet specific needs. The work presented in this thesis has provided experimental and basic theoretical tools allowing one to examine electro-optical physics - in particular, charge generation and recombination - in thin-film optoelectronic devices. As such, it has delivered important progress towards the realization of high-efficiency thin- and thick-junction functional devices, both critical requirements for achieving large-area and up-scaled optoelectronic applications.

Chapter 1 reviewed basic principles and fundamentals of excitonic optoelectronic device physics while pointing out the link between device efficiency and generation and recombination processes governing these systems.

In Chapter 2, the limitations on the sensitivity of external quantum efficiency [EQE] measurements were discussed. Different optical and electrical noise sources, such as parasitic stray light and photon noise of the light source, or shunt-limited thermal and electrical shot noise of the device under test, were carefully identified by probing the EQE of a model system under different electrical and light bias conditions. By minimization of these sensitivity-limiting factors, a dynamic range of $\sim 100 \mathrm{~dB}$ with a homebuilt EQE apparatus could be achieved, and photocurrents as small as fractions of a femto-amp could be detected. The superior dynamic range of the home-built EQE setup was achieved by using a high-performance, double-monochromator spectrophotometer Lambda950 as a low parasitic stray-light, broad wavelength light source, additional optical long-pass filters, high-gain current- and voltage pre-amplifiers with integrated low-noise voltage sources, and signal detection via lock-in method. The contribution of inter- and intra- molecular states to photocurrent generation in organic, inorganic, 
and perovskite solar cells could be directly observed, and additional sub-gap absorption features at photon energies well below the bandgap - derived from low-finesse cavity effects and sub-gap trap states - could be revealed.

Based upon the advancement in EQE sensitivity, a new approach for the accurate determination of charge generation quantum yields [CGY] in organic solar cells was introduced in Chapter 3. This thermal-kinetic rate approach is based upon temperaturedependent, and absorption-corrected ultra-sensitive EQE measurements. Non-fullerene acceptor- [NFA-] based organic solar cells were investigated, and near-unity CGYs were revealed in state-of-the-art, high-efficiency NFA photovoltaic devices. As such, an increase in CGY from 0.984 to 0.993 was found in PM6:BTP-eC9 and was directly compared to the performance, energetic, and optoelectronic properties of the related system PM6:Y6. Careful examination of charge transport via resistance-dependent photovoltage and double-injection current measurements accompanied by electro-optical simulations shed light on the subtle, but important, link between high CGYs and strongly suppressed Langevin recombination rates - the 400 times reduced Langevin recombination in PM6:Y6 was found to be even further suppressed to roughly 1000 times in PM6:BTPeC9. Motivated by these findings, high-efficiency thick-junction PM6:BTPeC9 solar cells were fabricated delivering above $16 \%$ power conversion efficiency - one of the best $300 \mathrm{~nm}$ thick-junction performances to date.

A newly developed measurement technique, that is, low-intensity integral time-of-flight [or, LIITOF], was introduced in Chapter 4. LIITOF is based upon the well-known integral time-of-flight method, but extended to the low-intensity regime and combined with device capacitance measurements. This simple and easy-to-use experimental technique allows one to measure small charge carrier densities derived from photon-pulse charge excitations in the photo-active layer sensitively. The theoretical framework of LIITOF was derived by one-dimensional drift-diffusion simulations. The technique was applied to organic and perovskite thin-film solar cells to probe the external generation efficiency as a function of applied bias voltage, and experimental findings were found to be in good agreement with results obtained via time-delayed collection field [TDCF] measurements conducted on the same devices. 


\section{SUMMARY AND OUTLOOK}

In Chapter 5, the competitive character between charge recombination and extraction defining the overall power conversion efficiency of every photovoltaic device was discussed. Supported by a drift-diffusion simulation model, the light intensity dependence of photocurrent [IPC] was reviewed in the presence of various charge loss mechanisms, such as trap-assisted Shockley-Read-Hall recombination, the built-up of space-charge due to imbalanced charge carrier mobilities, current limitation caused by series resistance, and bimolecular bulk recombination. On two different NFA solar cell model systems it was demonstrated, how IPC measurements, when performed sensitively over a broad range of light intensities, can be used to identify those predominant photocurrent loss mechanisms based upon their unique features.

The main body of the work described in Chapter 6 were IPC measurements realized on a large variety of fullerene and NFA acceptor-based organic solar cells including state-of-the-art, high-efficiency PM6:Y6 and PM6:BTP-eC9. First-order, trap-assisted recombination was found to be present in all investigated organic photovoltaic devices revealing the universal presence of sub-gap trap states in organic donor: acceptor blends. Careful examination of trap state energetics revealed trap depths lying between 0.35-0.6 eV having trap densities between $10^{16}-10^{17} \mathrm{~cm}^{-3}$. Combining sensitive IPC measurements with ultra-sensitive EQE measurements conducted on the model systems PCDTBT:PC ${ }_{70} \mathrm{BM}$ and PCDTBT:PC 70 BM:m-MTDATA [ $1 \%$ by mole] exposed the quantum efficiency loss-inducing and charge generation-contributing character of organic semiconductor trap states.

Based upon the work presented in this thesis, different challenges and questions for future work can be defined, which all seek to link the field of fundamental device physics with material synthesis and device engineering. As such, the question arises as to whether the exact origin of the universally present trap states in organic solar cells is of an intrinsic, or extrinsic nature and hence, if energetic, molecular-structural, and/or bulk-morphological properties can be modified to mitigate the photocurrent loss-inducing character of these sub-gap trap states. While a large number of studies in the literature - concentrating on the investigation of trap states in organic photovoltaic - focus merely on thin-film devices, changes in trap energetics with increasing active layer thickness should now be of significant interest, and could deliver important findings needed for the realization of large area organic photovoltaic. Despite the striking, 
recent success of NFA-based organic solar cells, fundamental relations between energetic offsets, activation energies for charge generation, and morphology-related charge transport properties need to be addressed in the future. In this regard, experimental techniques, such as sensitive IPC, EQE, and LIITOF, have proven to be able to significantly contribute to the photovoltaic community and to assist by completing the understanding of organic photovoltaic in general, and NFA-based application in particular. The theoretical concepts and experimental approaches to investigate charge generation and recombination in thin-film optoelectronic devices provided in this thesis may be also applied to other light-harvesting and photovoltaic material classes, such as indoor solar cells, photodetectors, or large-area, thick-junction photovoltaic devices. For the latter class of applications, the dominant series resistance of ordinarily interconnected modules, and the mismatch of photoactive layer and probe beam size requires further work on device- and experimental apparatus-related perspectives.

Addressing these challenges could further help to establish next generation applications based upon organic semiconductors as global commercial technologies. 
7. SUMMARY AND OUTLOOK 


\section{Appendix A}

\section{Device fabrication, statistics, and simulation model parameters}

The following part of the appendix provides details of materials, chemical names and molecular structures of organic semiconductors, and information on thin-film solar cell and single-carrier device fabrication. Statistics of photovoltaic device parameters, such as active layer thickness, fill factor, open-circuit voltage, short-circuit current density, and power conversion efficiency, are provided, and drift-diffusion simulation model parameters are listed.

This appendix is written based upon collaborative works (i) published by the author in the journals ACS Photonics [see Chapter 2] and Nature Communications [see Chapter 6], (ii) submitted to the journals Energy $\mathcal{E}$ Environmental Science [see Chapter 3] and Physical Review Applied [see Chapter 5], and (iii) currently under preparation for submission [see Chapter 4 ]. 


\section{A. DEVICE FABRICATION, STATISTICS, AND SIMULATION MODEL PARAMETERS}

\section{A.1 Chemical definitions}

PCDTBT: Poly[N-9 -heptadecanyl-2,7-carbazole-alt-5,5-(4, 7 -di-2-thienyl-2, , , 3 - benzothiadiazole)]

$\mathbf{P C}_{70}$ BM: [6,6]-phenyl-C71-butyric acid methyl ester

m-MTDATA: 4,4',4"'-Tris[(3-methylphenyl)phenylamino] triphenylamine

PTB7-Th: Poly[4,8-bis(5-(2-ethylhexyl)thiophen-2-yl)benzo[1,2-b;4,5-b']dithiophene2,6-diyl-alt-(4-(2-ethylhexyl)-3-fluorothieno[3,4-b]thiophene-)-2-carboxylate-2-6-diyl)]

PBDB-T: Poly[(2,6-(4,8-bis(5-(2-ethylhexyl)thiophen-2-yl)-benzo[1,2-b:4,5-b ] dithiophene)) - alt-(5,5-(1',3'-di-2-thienyl-5 ,7 -bis(2-ethylhexyl)benzo[1 ,2 -c:4 ,5 -c ]dithiophene4,8 -dione)]

ITIC: 3,9-bis(2-methylene-(3-(1,1-dicyanomethylene)-indanone))-5,5,11,11-tetrakis(4hexylphenyl)-dithieno[2,3-d:2,3 -d ]-s-indaceno[1,2-b:5,6-b ]dithiophene

IT-4F: 3,9-bis(2-methylene-((3-(1,1-dicyanomethylene)-6,7-difluoro)-indanone))-5,5,11,11tetrakis(4-hexylphenyl)-dithieno[2,3-d:2,3 -d ]-s-indaceno[1,2-b:5,6-b ] dithiophene

EH-IDTBR: has not been named. CAS number: 2102510-60-9

PM6: Poly[(2,6-(4,8-bis(5-(2-ethylhexyl-3-fluoro)thiophen-2-yl)-benzo[1,2-b:4,5-b ] dithiophene))-alt-(5,5-(1,3 -di-2-thienyl-5 ,7 -bis(2-ethylhexyl)benzo[1 ,2 -c:4 ,5 -c ] dithiophene-4,8-dione)]

Y6: 2,2 -((2Z,2 Z)-((12,13-bis(2-ethylhexyl)-3,9-diundecyl-12,13-dihydro-[1,2,5]thiadiazolo[

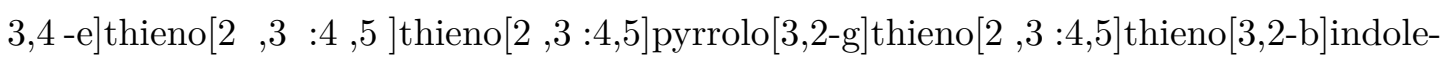
2,10-diyl)bis(methanylylidene))bis(5,6-difluoro-3-oxo-2,3-dihydro-1H-indene-2,1-diylidene)) dimalononitrile

BTP-eC9: 2,2'- [[12,13-Bis(2-butyloctyl)-12,13-dihydro-3,9-dinonylbisthieno[2", 3":4',5'] thieno [2',3':4,5]pyrrolo[3,2-e:2',3'-g] [2,1,3] benzothiadiazole-2,10-diyl] bis[methylidyne(5,6chloro-3-oxo-1H-indene-2,1(3H)-diylidene) ]] bis[propanedinitrile]

PEDOT:PSS: Poly(3,4-ethylenedioxythiophene) polystyrene sulfonate

PDINO: 2,9-bis[3-(dimethyloxidoamino)propyl]anthra[2,1,9-def:6,5,10-d e f ]diisoquinoline$1,3,8,10(2 \mathrm{H}, 9 \mathrm{H})$-tetrone 


\section{A.2 Molecular structures}
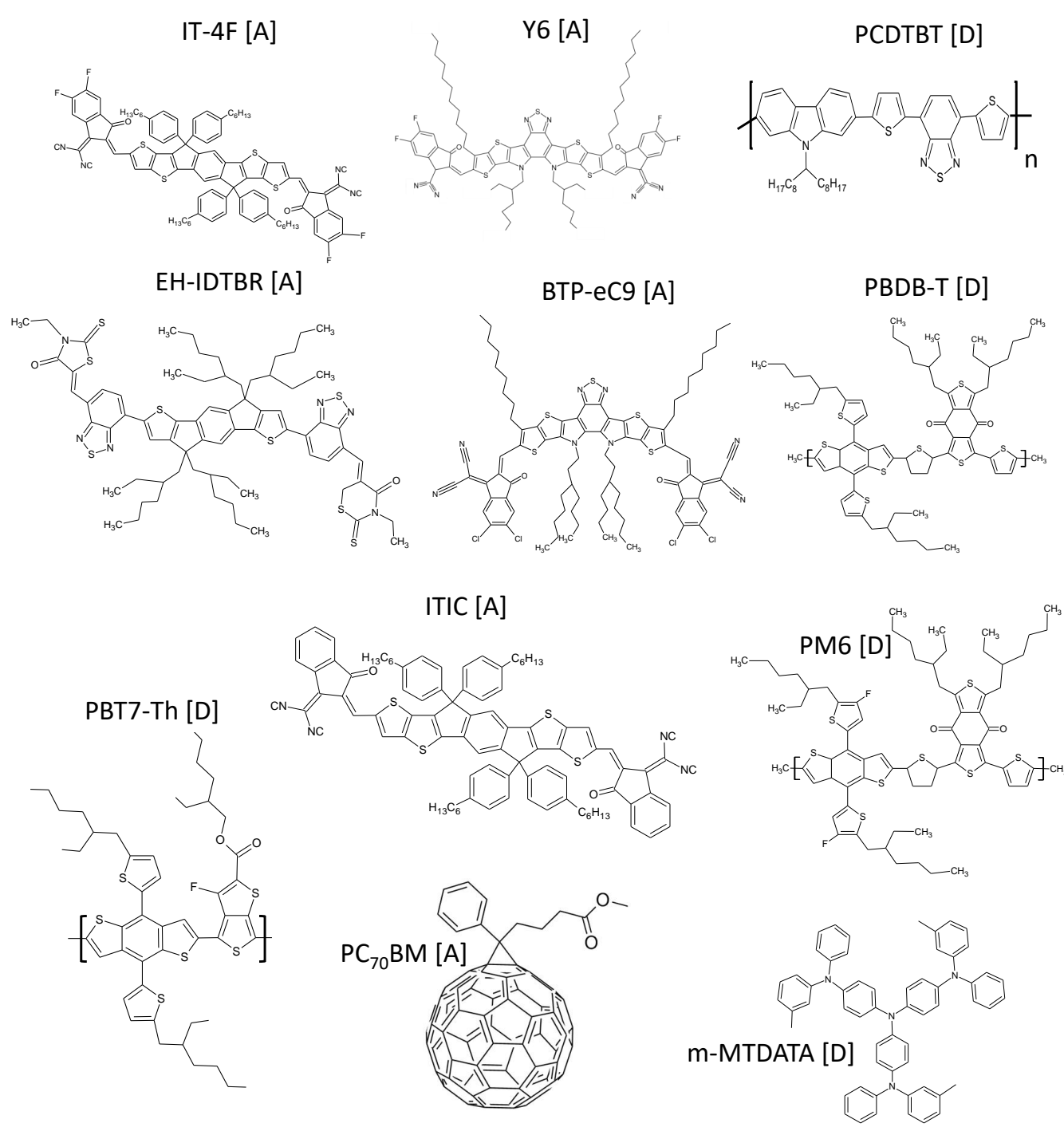

Figure A.1: Molecular structure of $\mathrm{PC}_{70} \mathrm{BM}, \mathrm{m}-\mathrm{MTDATA}$, PTB7-Th, PBDB-T, ITIC, IT-4F, EH-IDTBR, PM6, Y6, and BTP-eC9 acting either as an electron donating [D], or an electron accepting $[\mathrm{A}]$ organic semiconductor. 


\section{A. DEVICE FABRICATION, STATISTICS, AND SIMULATION MODEL PARAMETERS}

\section{A.3 Device fabrication}

\section{A.3.1 Inverted organic solar cell architecture}

PCDTBT:PC 70 BM (with and without m-MTDATA), PTB7-Th:ITIC, PBDBT:ITIC, PTB7-Th:PC 70 BM (with and without DIO), PBDB-T:IT-4F, PBDB-

T:EH-IDTBR, and PM6:IT-4F solar cells were fabricated with an inverted architecture (glass/ indium tin oxide (ITO)/ $\mathrm{ZnO} /$ active layer/ $\mathrm{MoO}_{3} / \mathrm{Ag}$ ). The commercial ITO patterned glass electrodes were cleaned by sonication in distilled water, acetone and 2-propanole in sequence each for $10 \mathrm{~min}$. The cleaned substrates were first dried with a stream of nitrogen and then transferred to a $100{ }^{\circ} \mathrm{C}$ hotplate, and further treated with an Oxygen plasma for 10 minutes. The $\mathrm{ZnO}$ performs as an electron transport layer and was prepared by dissolving $200 \mathrm{mg}$ zinc acetate dihydrate (purchased from Sigma-Aldrich) in 2-methoxyethanol $(2 \mathrm{~mL})$ using ethanolamine $(56 \mu \mathrm{L})$ as the stabilizer. The solution was stirred overnight under ambient conditions and spin-coated (4000 rpm for $30 \mathrm{~s}$ ) onto the ITO substrates and further annealed at $200{ }^{\circ} \mathrm{C}$ for 1 hour to obtain a thickness of approximately $30 \mathrm{~nm}$. Active layer deposition of the above systems was conducted by spin-casting, and their details are listed below. Subsequently, $7 \mathrm{~nm}$ of $\mathrm{MoO}_{3}$ and $100 \mathrm{~nm}$ of $\mathrm{Ag}$ were evaporated through a shadow mask in a vacuum chamber with $<10^{-6}$ mbar base pressure defining a $0.04 \mathrm{~cm}^{2}$ cell area for each pixel. Afterwards, devices were sealed with a cover glass using UV light-annealed glue from Bluefix. All the thicknesses of the above films are measured by ellipsometry.

Details of active layer depositions are as follows:

PCDTBT:PC 70 BM: PCDTBT and $\mathrm{PC}_{70} \mathrm{BM}$ were purchased from Ossila. A total concentration of $25 \mathrm{mg} \mathrm{mL} \mathrm{m}^{-1}$ in Chlorobenzene $(\mathrm{CB})$ with a donor: acceptor ratio of 1:4 was used to prepare the active layer solution. The solution was spin-coated at $1500 \mathrm{rpm}$ to form a film with the thickness around $100 \mathrm{~nm}$.

PCDTBT:PC 70 BM:m-MTDATA (1 \%): A total concentration of $25 \mathrm{mg} \mathrm{mL}^{-1}$ in $\mathrm{CB}$ with a PCDTBT:PC 70 BM:m-MTDATA weight ratio of 1:4:0.01 was used to prepare the active layer solution. The solution was spin-coated at $1500 \mathrm{rpm}$ to form a film with the thickness around $100 \mathrm{~nm}$. 
PTB7-Th:PC 70 BM (with DIO): PTB7-Th was purchased from Zhi-yan (Nanjing). A total concentration of $16 \mathrm{mg} \mathrm{mL}^{-1}$ in CB:DIO (97:3, v/v) with a donor: acceptor ratio of 1:1.5 was used to prepare the active layer solution. The solution was spin-coated at $600 \mathrm{rpm}$ to form a film with the thickness around $110 \mathrm{~nm}$.

PTB7-Th:PC ${ }_{70}$ BM (without DIO): A total concentration of $16 \mathrm{mg} \mathrm{mL}^{-1}$ in $\mathrm{CB}$ solution with a donor: acceptor ratio of 1:1.5 was used to prepare the active layer solution. The solution was spin-coated at $600 \mathrm{rpm}$ to form a film with the thickness around $110 \mathrm{~nm}$.

PTB7-Th:ITIC: ITIC was purchased from Zhi-yan (Nanjing). A total concentration of $14 \mathrm{mg} \mathrm{mL}^{-1}$ in CB:DIO (99:1, v/v) with a donor: acceptor ratio of 1:1.4 was used to prepare the active layer solution. The solution was spin-coated at $700 \mathrm{rpm}$ to form a film with the thickness around $100 \mathrm{~nm}$.

PBDB-T:ITIC: PBDB-T was purchased from Zhi-yan (Nanjing). A total concentration of $16 \mathrm{mg} \mathrm{mL}^{-1}$ in CB:DIO $(99: 1, \mathrm{v} / \mathrm{v})$ with a donor: acceptor ratio of 1:1 was used to prepare the active layer solution. The solution was spin-coated at $900 \mathrm{rpm}$ and further thermal annealed at $100{ }^{\circ} \mathrm{C}$ for $10 \mathrm{~min}$ to form a film with the thickness around $100 \mathrm{~nm}$.

PBDB-T:IT-4F: IT-4F was purchased from Solarmer (Beijing). A total concentration of $16 \mathrm{mg} \mathrm{mL}^{-1}$ in CB:DIO (99:1, v/v) with a donor: acceptor ratio of 1:1 was used to prepare the active layer solution. The solution was spin-coated at $900 \mathrm{rpm}$ and further thermal annealed at $100{ }^{\circ} \mathrm{C}$ for $10 \mathrm{~min}$ to form a film with the thickness around $100 \mathrm{~nm}$.

PBDB-T:EH-IDTBR: EH-IDTBR was purchased from Solarmer (Beijing). A total concentration of $16 \mathrm{mg} \mathrm{mL}^{-1}$ in CB:DIO $(99: 1, \mathrm{v} / \mathrm{v})$ with a donor: acceptor ratio of 1:1 was used to prepare the active layer solution. The solution was spin-coated at 900 rpm and further thermal annealed at $100{ }^{\circ} \mathrm{C}$ for $10 \mathrm{~min}$ to form a film with the thickness around $100 \mathrm{~nm}$. Note that for PBDB-T:EH-IDTBR thickness series [see Chapter 3], the films were adjusted by changing the concentration of the solution and spincoating speed (40 mg ml${ }^{-1}$ CB:DIO solution with $700 \mathrm{rpm}$ for $310 \mathrm{~nm}$ films, $35 \mathrm{mg}$ $\mathrm{ml}^{-1} \mathrm{CB}$ :DIO solution with $700 \mathrm{rpm}$ for $270 \mathrm{~nm}$ films, $30 \mathrm{mg} \mathrm{ml}^{-1} \mathrm{CB}$ :DIO solution with $700 \mathrm{rpm}$ for $200 \mathrm{~nm}$ films, $24 \mathrm{mg} \mathrm{ml}^{-1}$ CB:DIO solution with $700 \mathrm{rpm}$ for $170 \mathrm{~nm}$ films, $14 \mathrm{mg} \mathrm{ml}^{-1} \mathrm{CB}$ :DIO solution with $700 \mathrm{rpm}$ for $90 \mathrm{~nm}$ films, $10 \mathrm{mg} \mathrm{ml}^{-1} \mathrm{CB}$ :DIO solution with $700 \mathrm{rpm}$ for $50 \mathrm{~nm}$ films). 
PM6:IT-4F: A total concentration of $14 \mathrm{mg} \mathrm{mL}^{-1}$ in Chloroform (CF): DIO (99.5:0.5, $\mathrm{v} / \mathrm{v}$ ) with a donor: acceptor ratio of 1:1 was used to prepare the active layer solution. The solution was spin-coated at $3000 \mathrm{rpm}$ and further thermal annealed at $100{ }^{\circ} \mathrm{C}$ for $10 \mathrm{~min}$ to form a film with thickness around $100 \mathrm{~nm}$. 


\section{A.3.2 Conventional organic solar cell architecture}

PM6:ITIC, PBDB-T:Y6, PM6:Y6 and PM6:BTP-eC9 solar cells were fabricated with a conventional architecture (glass/ indium tin oxide (ITO)/ PEDOT:PSS/ active layer/ PDINO/ Ag). The commercial ITO patterned glass electrodes were cleaned by sonication in distilled water, acetone and 2-propanole in sequence each for $10 \mathrm{~min}$. The cleaned substrates were first dried with a stream of nitrogen and then transferred to a $100{ }^{\circ} \mathrm{C}$ hotplate, and further treated with an Oxygen plasma for 10 min. PEDOT:PSS solution was first diluted with the same volume of water and then cast at $4000 \mathrm{rpm}$ on ITO substrates followed by thermal annealing at $155{ }^{\circ} \mathrm{C}$ for 15 min to form a $10 \mathrm{~nm}$ film. Active layer deposition of the above systems was conducted by spin-casting, and their details are listed below. Subsequently, $1.5 \mathrm{mg} \mathrm{ml}^{-1}$ PDINO solution was spin-coated on active layer film at $2000 \mathrm{rpm}$ to form $10 \mathrm{~nm}$ films, and $100 \mathrm{~nm}$ of $\mathrm{Ag}$ were evaporated through a shadow mask in a vacuum chamber with $<10^{-6}$ mbar base pressure. Afterwards, devices were sealed with a cover glass using UV light-annealed glue from Bluefix. All the thickness of the above films are measured by ellipsometry.

Details of active layer depositions are as follows:

PBDB-T:Y6: Y6 was purchased from Solarmer (Beijing). A total concentration of $12 \mathrm{mg} \mathrm{mL}{ }^{-1}$ in CF: 1-chloronaphthalene (99.5:0.5, v/v) with a donor: acceptor ratio of 1:1.2 was used to prepare the active layer solution. The solution was spin-coated at $2000 \mathrm{rpm}$ and further thermal annealed at $100{ }^{\circ} \mathrm{C}$ for $10 \mathrm{~min}$ to form a film with the thickness around $100 \mathrm{~nm}$.

PM6:ITIC: PM6 was purchased from Solarmer (Beijing). A total concentration of $14 \mathrm{mg} \mathrm{mL}{ }^{-1}$ in CF:DIO $(99: 1, \mathrm{v} / \mathrm{v})$ with a donor: acceptor ratio of 1:1 was used to prepare the active layer solution. The solution was spin-coated at $3000 \mathrm{rpm}$ and further thermal annealed at $100{ }^{\circ} \mathrm{C}$ for $10 \mathrm{~min}$ to form a film with the thickness around $100 \mathrm{~nm}$. Note that for PM6:ITIC thickness series [see Chapter 3], the films were adjusted by changing the concentration of the solution and spin-coating speed (40 $\mathrm{mg} \mathrm{ml}^{-1} \mathrm{CF}$ :DIO solution with $2000 \mathrm{rpm}$ for $660 \mathrm{~nm}$ films, $36 \mathrm{mg} \mathrm{ml}^{-1} \mathrm{CF}$ :DIO solution with $2000 \mathrm{rpm}$ 


\section{A. DEVICE FABRICATION, STATISTICS, AND SIMULATION MODEL PARAMETERS}

for $470 \mathrm{~nm}$ films, $32 \mathrm{mg} \mathrm{ml}^{-1} \mathrm{CF}$ :DIO solution with $2000 \mathrm{rpm}$ for $2880 \mathrm{~nm}$ films, $25 \mathrm{mg}$

$\mathrm{ml}^{-1} \mathrm{CF}$ :DIO solution with $2000 \mathrm{rpm}$ for $180 \mathrm{~nm}$ films, $20 \mathrm{mg} \mathrm{ml}^{-1}$ CF:DIO solution with $3000 \mathrm{rpm}$ for $130 \mathrm{~nm}$ films, $14 \mathrm{mg} \mathrm{ml}^{-1} \mathrm{CF}$ :DIO solution with $3000 \mathrm{rpm}$ for 90 nm films, $12 \mathrm{mg} \mathrm{ml}^{-1} \mathrm{CF}$ :DIO solution with $3000 \mathrm{rpm}$ for 60 films).

PM6:Y6: A total concentration of $16 \mathrm{mg} \mathrm{mL}^{-1}$ in CF: 1-chloronaphthalene (99.5:0.5, $\mathrm{v} / \mathrm{v}$ ) with a donor: acceptor ratio of 1:1.2 was used to prepare the active layer solution. The solution was spin-coated at $3000 \mathrm{rpm}$ and further thermal annealed at $100{ }^{\circ} \mathrm{C}$ for $10 \mathrm{~min}$ to form a film with the thickness around $100 \mathrm{~nm}$. Note that for PM6:Y6 thickness series [see Chapter 3], the films were adjusted by changing the concentration of the solution and the spin-coating speed $\left(40 \mathrm{mg} \mathrm{ml}^{-1} \mathrm{CF}\right.$ :CN solution with $4000 \mathrm{rpm}$ for $390 \mathrm{~nm}, 35 \mathrm{mg} \mathrm{ml}^{-1} \mathrm{CF}$ :DIO solution with $4000 \mathrm{rpm}$ for $310 \mathrm{~nm}, 30 \mathrm{mg} \mathrm{ml}^{-1} \mathrm{CF}$ :DIO solution with $4000 \mathrm{rpm}$ for $260 \mathrm{~nm}, 25 \mathrm{mg} \mathrm{ml}^{-1}$ CF:DIO solution with $4000 \mathrm{rpm}$ for $190 \mathrm{~nm}, 20 \mathrm{mg} \mathrm{ml}^{-1} \mathrm{CF}$ :DIO solution with $4000 \mathrm{rpm}$ for $160 \mathrm{~nm}, 16 \mathrm{mg} \mathrm{ml}^{-1} \mathrm{CF}$ :DIO solution with $2000 \mathrm{rpm}$ for $109 \mathrm{~nm}, 5 \mathrm{mg} \mathrm{ml}{ }^{-1} \mathrm{CF}$ :DIO solution with $2000 \mathrm{rpm}$ for 30 $\mathrm{nm})$.

PM6:BTP-eC9: BTP-eC9 was purchased from Solarmer (Beijing). A total concentration of $16 \mathrm{mg} \mathrm{mL}^{-1}$ in CF: DIO (99.5:0.5, v/v) with a donor: acceptor ratio of 1:1.2 was used to prepare the active layer solution. The solution was spin-coated at $3000 \mathrm{rpm}$ and further thermal annealed at $100{ }^{\circ} \mathrm{C}$ for $10 \mathrm{~min}$ to form a film with the thickness around $100 \mathrm{~nm}$. Note that for PM6:BTP-eC9 thickness series [see Chapter 3], films were adjusted by changing the concentration of the solution and the spin-coating speed (40 mg ml ${ }^{-1}$ CF:DIO solution with $2000 \mathrm{rpm}$ for $446 \mathrm{~nm}, 35 \mathrm{mg} \mathrm{ml}^{-1} \mathrm{CF}$ :DIO solution with $2000 \mathrm{rpm}$ for $340 \mathrm{~nm}, 30 \mathrm{mg} \mathrm{ml}^{-1}$ CF:DIO solution with $2000 \mathrm{rpm}$ for $293 \mathrm{~nm}$, $25 \mathrm{mg} \mathrm{ml}^{-1} \mathrm{CF}$ :DIO solution with $2000 \mathrm{rpm}$ for $260 \mathrm{~nm}, 20 \mathrm{mg} \mathrm{ml}^{-1} \mathrm{CF}$ :DIO solution with $2000 \mathrm{rpm}$ for $160 \mathrm{~nm}, 16 \mathrm{mg} \mathrm{ml}^{-1} \mathrm{CF}$ :DIO solution with $3000 \mathrm{rpm}$ for $90 \mathrm{~nm}, 12$ $\mathrm{mg} \mathrm{ml}{ }^{-1} \mathrm{CF}$ :DIO solution with $3000 \mathrm{rpm}$ for $60 \mathrm{~nm}$ ).

The PCDTBT:PC $70 \mathrm{BM}$ and neat PCDTBT thin-film solar cells from Chapter 4 were fabricated by Dr. J. Kurpiers. A detailed description of the PCDTBT:PC ${ }_{70} \mathrm{BM}$ device fabrication can be found in [159]. For the neat PCDTBT device, only the active layer preparation was different to [159], where $20 \mathrm{mg} \mathrm{ml}^{1} \mathrm{PCDTBT}$ was dissolved in CB and spin-coated at $2000 \mathrm{rpm}$. 


\section{A.3.3 Perovskite solar cells}

The triple cation perovskite solar cell [see Chapter 4] was fabricated by Dr. J. Kurpiers according to the recipe provided in [202] - the following device fabrication description is written based upon it.

PEDOT:PSS (purchased from Heraeus Celivious 4083) was spin-coated at $2000 \mathrm{rpm}$ for $40 \mathrm{~s}$ with an acceleration of $2000 \mathrm{rpm} \mathrm{s}^{-1}$, before annealing for $15 \mathrm{~min}$ at $150{ }^{\circ} \mathrm{C}$ on a hotplate. P3HT (purchased from Sigma-Aldrich, molecular weight $\sim 27$ 000) was dissolved in $3 \mathrm{mg} \mathrm{mL} \mathrm{mL}^{-1} \mathrm{DCB}$ solution and spincoated for $30 \mathrm{~s}$ at $3000 \mathrm{rpm}$ with an acceleration of $3000 \mathrm{rpm} \mathrm{s}^{-1}$. The P3HT layer was annealed for $10 \mathrm{~min}$ at $100{ }^{\circ} \mathrm{C}$, before treated with oxygen for $5 \mathrm{~s}$. PolyTPD (purchased from Ossila) was dissolved in $1.5 \mathrm{mg} \mathrm{mL} \mathrm{m}^{-1} \mathrm{DCB}$ solution and spin-coated at $6000 \mathrm{rpm}$ for $30 \mathrm{~s}$ with an acceleration of $2000 \mathrm{rpm} \mathrm{s}^{-1}$. Subsequently, the PolyTPD layer was annealed on a hotplate for $10 \mathrm{~min}$ at $100{ }^{\circ} \mathrm{C}$. PTAA (purchased from Sigma-Aldrich) was spincoated from a $1.5 \mathrm{mg} \mathrm{mL} \mathrm{mL}^{-1}$ Toluene solution for $30 \mathrm{~s}$ at $6000 \mathrm{rpm}$ with an acceleration of $2000 \mathrm{rpm} \mathrm{s}^{-1}$ to form a $\sim 10 \mathrm{~nm}$ hick film. Onto the spinning substrate at $5000 \mathrm{rpm}$ for $20 \mathrm{~s}, 60 \mu \mathrm{L}$ solution of PFN-P2 (0.5 mg mL $\mathrm{m}^{-1}$ in methanol) was added, before annealing for $10 \mathrm{~min}$ on a hotplate at $100{ }^{\circ} \mathrm{C}$. The perovskite (triple cation) solution was prepared by mixing 1.3 $\mathrm{M} \mathrm{FAPbI}_{3}$ and 1.3 $\mathrm{M} \mathrm{MAPbBr}_{3}$ perovskite solution in a ratio of 83:17 in DMF:DMSO (4:1) solution. Note that in the following, this final solution is referred to as 'MAFA' solution. The $1.3 \mathrm{M} \mathrm{FAPbI}_{3}$ solution was prepared by dissolving FAI (722 mg) and $\mathrm{PbI}_{2}(2130 \mathrm{mg}$ ) in $2.8 \mathrm{~mL} \mathrm{DMF}$ and $0.7 \mathrm{~mL}$ DMSO (note there is a $10 \%$ excess of $\mathrm{PbI}_{2}$ ). The $1.3 \mathrm{M} \mathrm{MAPbBr}_{3}$ solution, in turn, was made by dissolving $\mathrm{MABr}$ (470 mg) and $\mathrm{PbBr}_{2}(1696 \mathrm{mg}$ ) in $2.8 \mathrm{~mL} \mathrm{DMF}$ and $0.7 \mathrm{~mL}$ DMSO (note that there is a $10 \%$ excess of $\mathrm{PbBr}_{2}$ ). $40 \mu \mathrm{L}$ a $1.2 \mathrm{M}$ CsI solution in DMSO (389 mg CsI in $1 \mathrm{~mL}$ DMSO) was then mixed with $960 \mu \mathrm{L}$ the MAFA solution. The final perovskite stoichiometry was $\left(\mathrm{CsPbI}_{3}\right)_{0.05}\left[\left(\mathrm{FAPbI}_{3}\right)_{0.83}\left(\mathrm{MAPbBr}_{3}\right)_{0.17}\right]_{0.95}$ in solution, which was spincoated for $35 \mathrm{~s}$ at $4000 \mathrm{rpm}$ with an acceleration of $1300 \mathrm{rpm} \mathrm{s}^{-1}$. Note that $300 \mu \mathrm{L}$ EA solution was used $10 \mathrm{~s}$ after the spinning process started, to wash the spinning substrates for about $1 \mathrm{~s}$. The anti-solvent was placed right in the centre of the spinning film. The perovskite film was then annealed on a hotplate for $60 \mathrm{~min}$ at $100{ }^{\circ} \mathrm{C}$, before transferring the sample to an evaporation chamber. Under vacuum with a base pressure of $10^{-7}$ 


\section{A. DEVICE FABRICATION, STATISTICS, AND SIMULATION MODEL PARAMETERS}

mbar, $30 \mathrm{~nm}$ of $\mathrm{C}_{60}$ and $10 \mathrm{~nm}$ of $\mathrm{LiF}$ were evaporated at $0.1 \AA_{\mathrm{s}}^{-1}$ and $0.03 \AA_{\mathrm{s}}^{-1}$. The device was then completed by transferring the sample to another evaporation chamber, where under vacuum with base pressure of $10^{-7}$ mbar, $8 \mathrm{~nm}$ of BCP (purchased from Sigma-Aldrich) and $100 \mathrm{~nm}$ of copper (purchased from Sigma-Aldrich) were evaporated at $0.2 \AA_{\mathrm{s}}^{-1}$ and $0.6 \AA \mathrm{s}^{-1}$. 


\section{A.3.4 Single-carrier organic devices}

PTB7-Th:PC ${ }_{70} B M$ hole-only and electron-only devices: The device structures of hole-only and electron-only devices were: ITO/ PEDOT:PSS/ active layer/ $\mathrm{MoO}_{3}$ / $\mathrm{Ag}$ and ITO $\mathrm{ZnO} /$ active layer/ PDINO/ Ag respectively. For the hole-only devices, $40 \mathrm{~nm}$ PEDOT:PSS films were cast on clean ITO substrate. For the deposition of the PTB7-Th: PC $_{70} \mathrm{BM}$ active layer, $1400 \mathrm{~nm}$ PTB7-Th:PC $70 \mathrm{BM}$ (1:1.5, w/w) film was spin-coated (500 rpm) on PEDOT:PSS or ZnO substrates from $\mathrm{CB}$ solutions (50mg $\mathrm{mL}^{-1}$ with 3 vol\% DIO) and further rinsed with $80 \mu \mathrm{L}$ of methanol at $4000 \mathrm{rpm}$ for $20 \mathrm{~s}$. Following this, $7 \mathrm{~nm} \mathrm{MoO}$ and $100 \mathrm{~nm} \mathrm{Ag}$ were thermally evaporated to form a cathode under high vacuum. For the electron-only device, a $30 \mathrm{~nm} \mathrm{ZnO}$ film was cast on clean ITO substrates. Subsequently, the active layer was cast onto the $\mathrm{ZnO}$ substrate with the same method as the hole-only device. Following this, an $8 \mathrm{~nm}$ PDINO film was spin-coated (3000 rpm) from methanol solution $\left(1 \mathrm{mg} \mathrm{mL}^{-1}\right)$, and $100 \mathrm{~nm}$ of Ag thermally evaporated on PDINO to form an anode.

PBDB-T:ITIC hole-only and electron-only devices: The device structures of the hole-only and electron-only devices, and the deposition methods of PEDOT:PSS, $\mathrm{ZnO}, \mathrm{PDINO}, \mathrm{MoO}_{3}$ and $\mathrm{Ag}$ were the same as the PTB7-Th:PC $\mathrm{P}_{0} \mathrm{BM}$ hole-only and electron-only devices. For the deposition of the PBDB-T:ITIC active layer, a $800 \mathrm{~nm}$ PBDB-T:ITIC (1:1, w/w) film was spin-coated (1200 rpm) on PEDOT:PSS or ZnO substrates from CB solutions (50 $\mathrm{mg} \mathrm{mL}^{-1}$ with $\left.1 \mathrm{vol} \% \mathrm{DIO}\right)$ and further thermal annealed at $100{ }^{\circ} \mathrm{C}$ for $10 \mathrm{~min}$.

PCDTBT:PC ${ }_{70} \mathrm{BM}$ hole-only and electron-only devices: The device structures of the hole-only and electron-only devices, and the deposition methods of PEDOT:PSS, $\mathrm{ZnO}, \mathrm{PDINO}, \mathrm{MoO}_{3}$ and $\mathrm{Ag}$ were the same as the PTB7-Th:PC $70 \mathrm{BM}$ hole-only and electron-only devices. For the deposition of the PCDTBT:PC ${ }_{70} \mathrm{BM}$ active layer, 1100 nm PCDTBT:PC ${ }_{70} \mathrm{BM}(1: 4, \mathrm{w} / \mathrm{w})$ film was spin-coated (500 rpm) on PEDOT:PSS or $\mathrm{ZnO}$ substrates from $\mathrm{CB}$ solutions $\left(50 \mathrm{mg} \mathrm{mL}^{-1}\right)$. 


\section{A. DEVICE FABRICATION, STATISTICS, AND SIMULATION MODEL PARAMETERS}

\section{A.4 Device statistics and simulation model parameters}

\begin{tabular}{|c|c|c|c|c|c|}
\hline Device & $E_{\mathrm{H}, \mathrm{D}}[\mathrm{eV}]$ & $E_{\mathrm{L}, \mathrm{A}}[\mathrm{eV}]$ & $d[\mathrm{~nm}]$ & $\Delta_{\mathrm{t}}$ in $[\mathrm{eV}]$ & $N_{\mathrm{t}}\left[\mathrm{cm}^{-3}\right]$ \\
\hline A & $5.21[203]$ & 3.89 204 & 110 & 0.46 & $4 \times 10^{16}$ \\
\hline B & $5.3[195]$ & $3.9[205]$ & 80 & 0.48 & $5 \times 10^{16}$ \\
\hline $\mathrm{C}$ & $5.24[206$ & $3.9[205$ & 110 & 0.44 & $3 \times 10^{16}$ \\
\hline D & $5.21[203$ & $3.89[204]$ & 110 & 0.51 & $4 \times 10^{16}$ \\
\hline $\mathrm{E}$ & $5.24[206$ & $3.89[204$ & 90 & 0.38 & $5 \times 10^{16}$ \\
\hline $\mathrm{F}$ & 5.21 [203] & 4.14 & 110 & 0.45 & $3 \times 10^{16}$ \\
\hline G & 5.45207 & 4.14204 & 110 & 0.45 & $3 \times 10^{16}$ \\
\hline $\mathrm{H}$ & 5.45207 & 4.1 108 & 100 & 0.44 & $4 \times 10^{16}$ \\
\hline I & 5.45207 & 4.1108 & 100 & 0.40 & $4 \times 10^{16}$ \\
\hline $\mathrm{J}$ & 5.45207 & $3.89[204$ & 110 & 0.46 & $4 \times 10^{16}$ \\
\hline K & 5.21 [203] & $4.1[108$ & 90 & 0.42 & $5 \times 10^{16}$ \\
\hline $\mathrm{L}$ & $5.24[206]$ & $3.9[205$ & 90 & 0.45 & $3 \times 10^{16}$ \\
\hline M & $5.3[195$ & $3.9[205]$ & 80 & 0.37 & $7 \times 10^{16}$ \\
\hline
\end{tabular}

Table A.1: Energy levels of donor HOMO $\left[E_{\mathrm{H}, \mathrm{D}}\right]$ and acceptor LUMO $\left[E_{\mathrm{L}, \mathrm{A}}\right]$, active layer thicknesses $[d]$, trap depths $\left[\Delta_{\mathrm{t}, \mathrm{IPC}}\right]$ and densities $\left[N_{\mathrm{t}}\right]$ of the following organic solar cells: A [PBDB-T:ITIC], B [PCDTBT:PC 70 BM], C [PTB7-Th:PC ${ }_{70} \mathrm{BM}$ (with DIO)], D [PBDBT:EH-IDTBR], E [PTB7-Th:ITIC], F [PBDB-T:IT-4F], G [PM6:IT-4F], H [PM6:Y6], I [PM6:BTP-eC9], J [PM6:ITIC], K [PBDB-T:Y6], L [PTB7-Th:PC ${ }_{70} \mathrm{BM}$ (without DIO)], and $\mathrm{M}$ [PCDTBT:PC ${ }_{70}$ BM:m-MTDATA ( $1 \%$ by mole)].

\begin{tabular}{c||c|c|c|c}
\hline Device & $V_{\mathrm{oc}}[\mathrm{V}]$ & $\mathrm{FF}[\%]$ & $J_{\mathrm{sc}}\left[\mathrm{mA} \mathrm{cm}^{2}\right]$ & $\mathrm{PCE}_{\mathrm{avg}}\left(\mathrm{PCE}_{\max }\right)$ in $[\%]$ \\
\hline $\mathrm{A}$ & $0.90 \pm 0.01$ & $63.4 \pm 0.5$ & $10.7 \pm 0.2$ & $6.2 \pm 0.1(6.4)$ \\
$\mathrm{B}$ & $0.58 \pm 0.01$ & $35.2 \pm 1.2$ & $1.5 \pm 0.1$ & $0.32 \pm 0.1(0.34)$ \\
$\mathrm{C}$ & $0.70 \pm 0.01$ & $44.5 \pm 0.7$ & $14.3 \pm 0.3$ & $4.5 \pm 0.3(6.1)$ \\
$\mathrm{D}$ & $0.76 \pm 0.01$ & $65.3 \pm 0.4$ & $17.0 \pm 0.3$ & $8.6 \pm 0.3(9.1)$ \\
$\mathrm{E}$ & $0.99 \pm 0.01$ & $57.7 \pm 1.0$ & $10.2 \pm 0.2$ & $5.7 \pm 0.2(6.0)$ \\
$\mathrm{F}$ & $0.72 \pm 0.01$ & $75.0 \pm 0.7$ & $18.5 \pm 0.4$ & $9.6 \pm 0.2(9.9)$ \\
$\mathrm{G}$ & $0.83 \pm 0.01$ & $76.0 \pm 0.6$ & $19.4 \pm 0.4$ & $12.2 \pm 0.2(12.5)$ \\
$\mathrm{H}$ & $0.90 \pm 0.01$ & $68.5 \pm 1.1$ & $15.5 \pm 0.5$ & $9.3 \pm 0.4(9.7)$ \\
$\mathrm{I}$ & $0.97 \pm 0.01$ & $61.2 \pm 1.2$ & $16.2 \pm 0.6$ & $9.2 \pm 0.3(9.6)$ \\
$\mathrm{J}$ & $0.81 \pm 0.01$ & $65.3 \pm 0.5$ & $14.2 \pm 0.3$ & $7.6 \pm 0.2(7.9)$ \\
$\mathrm{K}$ & $0.74 \pm 0.01$ & $61.4 \pm 0.9$ & $24.0 \pm 0.4$ & $10.9 \pm 0.4(11.5)$ \\
$\mathrm{L}$ & $0.84 \pm 0.01$ & $76.0 \pm 1.0$ & $24.2 \pm 0.4$ & $15.2 \pm 0.3(15.6)$ \\
$\mathrm{M}$ & $0.84 \pm 0.01$ & $77.5 \pm 1.0$ & $24.3 \pm 0.4$ & $15.8 \pm 0.4(16.4)$ \\
\hline
\end{tabular}

Table A.2: Photovoltaic parameters $\left[V_{\mathrm{oc}}, \mathrm{FF}, J_{\mathrm{sc}}\right.$, and $\left.\mathrm{PCE}\right]$ of the following organic solar cells: A [PCDTBT:PC ${ }_{70} \mathrm{BM}$ ], B [PCDTBT:PC ${ }_{70} \mathrm{BM}$ :m-MTDATA ( $\%$ by mole)], C [PTB7-Th:PC 70 BM (without DIO)], D [PTB7-Th:PC ${ }_{70} \mathrm{BM}$ (with DIO)], E [PBDB-T:EHIDTBR], F [PBDB-T:IT-4F], G [PM6:IT-4F], H [PBDB-T:ITIC], I [PM6:ITIC], J [PTB7Th:ITIC], K [PBDB-T:Y6], L [PM6:Y6], and M [PM6:BTP-eC9]. 


\begin{tabular}{c||c|c|c|c}
\hline Parameter & Figure 6.1 & $\mathrm{~A}$ & $\mathrm{~B}$ & $\mathrm{C}$ \\
\hline$E_{\mathrm{g}}, \mathrm{eV}$ & 1.30 & 1.46 & 1.31 & 1.25 \\
$\varepsilon$ & 3.5 & 3.5 & 3.5 & 3.5 \\
$N_{\text {eff }}, \mathrm{cm}^{-3}$ & $10^{20}$ & $10^{20}$ & $10^{20}$ & $10^{20}$ \\
$d, \mathrm{~nm}$ & 100 & 80 & 110 & 100 \\
$\bar{G}, \mathrm{~cm}^{-3} \mathrm{~s}^{-1}$ & $10^{22}$ & $8 \times 10^{21}$ & $9.5 \times 10^{21}$ & $1.59 \times 10^{22}$ \\
$\mu, \mathrm{cm}^{2} \mathrm{~V}^{-1} \mathrm{~s}^{-1}$ & $10^{-4}$ & $5 \times 10^{-4}$ & $5 \times 10^{-3}$ & $1 . .5 \times 10^{-3}$ \\
$R_{\text {band-to-band }}, \mathrm{cm}^{3} \mathrm{~s}^{-1}$ & $10^{-11}$ & $2.6 \times 10^{-10}$ & $5.2 \times 10^{-11}$ & $1.6 \times 10^{-12}$ \\
$N_{\mathrm{t}}, \mathrm{cm}^{-3}$ & $10^{-17}$ & $2.5 \times 10^{-16}$ & $8 \times 10^{-16}$ & $1.6 \times 10^{-17}$ \\
$\Delta_{\mathrm{t}}, \mathrm{eV}$ & 0.4 & 0.49 & 0.49 & 0.41 \\
$\tau_{\mathrm{SRH}}, \mu \mathrm{S}$ & 1.0 & 0.31 & 0.24 & 4.0 \\
$E_{\text {injection }}, \mathrm{eV}$ & 0.20 & 0.20 & 0.0 & 0.05 \\
$R_{\text {shunt }}, \Omega \mathrm{cm}^{2}$ & $4 \times 10^{5}$ & $2 \times 10^{4}$ & $1.7 \times 10^{3}$ & $7 \times 10^{5}$ \\
$R_{\text {series }}, \Omega \mathrm{cm}^{2}$ & - & 1.0 & 1.0 & 1.0 \\
\hline
\end{tabular}

Table A.3: Parameters used for the drift diffusion simulations shown in Fig. 6.1, and for devices A [PCDTBT:PC ${ }_{70} \mathrm{BM}$ ], B [PTB7-Th:PC $\left.{ }_{70} \mathrm{BM}\right]$, and $\mathrm{C}$ [PM6:BTP-eC9] shown in Fig. 6.2: energy level gap $\left[E_{\mathrm{g}}\right]$, relative permittivity $[\varepsilon]$, effective density of states $\left[N_{\text {eff }}\right]$, active layer thickness $[d]$, average generation rate at 1 sun $[\bar{G}]$, mobility $[\mu]$, band-to-band recombination rate $\left[R_{\text {band-to-band }}\right]$, trap density $\left[N_{\mathrm{t}}\right]$, trap depth $\left[\Delta_{\mathrm{t}}\right]$, Shockley-Read-Hall carrier lifetime $\left[\tau_{\mathrm{SRH}}\right]$, injection barrier $\left[E_{\text {injection }}\right]$, shunt resistance $\left[R_{\text {shunt }}\right]$, and series resistance $\left[R_{\text {series }}\right]$.

\begin{tabular}{c||c|c}
\hline Parameter & PM6:Y6 & PM6:BTP-eC9 \\
\hline$T, \mathrm{~K}$ & 300 & 300 \\
$\varepsilon$ & 3.4 & 3.4 \\
$\mu_{\mathrm{n}}, \mathrm{cm}^{2} \mathrm{~V}^{-1} \mathrm{~s}^{-1}$ & $1.2 \times 10^{-3}$ & $1.5 \times 10^{-3}$ \\
$\mu_{\mathrm{p}}, \mathrm{cm}^{2} \mathrm{~V}^{-1} \mathrm{~s}^{-1}$ & $2 \times 10^{-4}$ & $4 \times 10^{-4}$ \\
$\gamma$ & $2.5 \times 10^{-4}$ & $10^{-3}$ \\
$E_{\mathrm{g}, \mathrm{DA}}, \mathrm{eV}$ & 1.22 & 1.24 \\
$V_{\mathrm{bi}}, \mathrm{V}$ & 1.02 & 1.04 \\
$N_{\mathrm{N}, \mathrm{V}}, \mathrm{cm}^{-3}$ & $10^{20}$ & $10^{20}$ \\
$E_{\text {injection }}, \mathrm{eV}$ & 0.1 & 0.1 \\
\hline
\end{tabular}

Table A.4: Input parameters for electro-optical device simulations shown in Fig. 3.8: temperature $[T]$, relative permittivity $[\varepsilon]$, electron mobility $\left[\mu_{\mathrm{n}}\right]$, hole mobility $\left[\mu_{\mathrm{p}}\right]$, Langevin reduction factor $[\gamma]$, electrical bandgap $\left[E_{\mathrm{g}, \mathrm{DA}}\right]$, built-in voltage $\left[V_{\mathrm{bi}}\right]$, effective density of states in conduction and valence band $\left[N_{\mathrm{N}, \mathrm{V}}\right]$, and injection barrier for majority carriers at the contact $\left[E_{\text {injection }}\right]$. 


\section{A. DEVICE FABRICATION, STATISTICS, AND SIMULATION MODEL PARAMETERS}

\begin{tabular}{c||c|c|c|c}
\hline Thickness $[\mathrm{nm}]$ & $\mathrm{FF}[\%]$ & $J_{\mathrm{sc}}\left[\mathrm{mA} \mathrm{cm}^{2}\right]$ & $V_{\mathrm{oc}}[\mathrm{V}]$ & $\mathrm{PCE}_{\max }\left(\mathrm{PCE}_{\mathrm{avg}}\right)[\%]$ \\
\hline 30 & 78.9 & 14.1 & 0.81 & $9.1(8.7 \pm 0.4)$ \\
109 & 76.6 & 25.1 & 0.81 & $15.3(15.2 \pm 0.4)$ \\
160 & 70.4 & 25.2 & 0.81 & $14.3(13.9 \pm 0.4)$ \\
190 & 56.3 & 25.4 & 0.80 & $13.4(13.2 \pm 0.2)$ \\
260 & 61.9 & 26.7 & 0.80 & $13.2(12.8 \pm 0.4)$ \\
310 & 61.4 & 26.8 & 0.80 & $13.1(12.6 \pm 05)$ \\
390 & 55.7 & 23.8 & 0.78 & $10.5(9.3 \pm 1.2)$ \\
\hline
\end{tabular}

Table A.5: Photovoltaic parameters [open-circuit voltage $\left(V_{\mathrm{oc}}\right)$, fill factor $(\mathrm{FF})$, shortcircuit current density $\left(J_{\mathrm{sc}}\right)$, and power-conversion efficiency (PCE)] of PM6:Y6 devices with different active layer thicknesses varying between $30 \mathrm{~nm}$ and $390 \mathrm{~nm}$. Information of the current density versus voltage measurements are provided in Box 3.1.

\begin{tabular}{c||c|c|c|c}
\hline Thickness $[\mathrm{nm}]$ & $\mathrm{FF}[\%]$ & $J_{\mathrm{sc}}\left[\mathrm{mA} \mathrm{cm}^{2}\right]$ & $V_{\mathrm{oc}}[\mathrm{V}]$ & $\mathrm{PCE}_{\max }\left(\mathrm{PCE}_{\mathrm{avg}}\right)[\%]$ \\
\hline 60 & 78.5 & 24.1 & 0.83 & $15.7(15.3 \pm 0.2)$ \\
90 & 79.1 & 25.7 & 0.84 & $17.1(16.7 \pm 0.2)$ \\
160 & 76.9 & 25.6 & 0.83 & $16.4(16.2 \pm 0.2)$ \\
200 & 72.3 & 27.7 & 0.83 & $16.5(16.1 \pm 0.3)$ \\
260 & 71.8 & 27.8 & 0.82 & $16.4(15.9 \pm 0.3)$ \\
293 & 71.3 & 27.7 & 0.82 & $16.2(15.7 \pm 0.4)$ \\
340 & 66.1 & 27.2 & 0.82 & $14.8(14.3 \pm 0.5)$ \\
446 & 59.9 & 26.8 & 0.82 & $13.1(12.4 \pm 0.7)$ \\
\hline
\end{tabular}

Table A.6: Photovoltaic parameters [open-circuit voltage $\left(V_{\mathrm{oc}}\right)$, fill factor $(\mathrm{FF})$, shortcircuit current density $\left(J_{\mathrm{sc}}\right)$, and power-conversion efficiency (PCE)] of PM6:BTP-eC9 devices with different active layer thicknesses varying between $60 \mathrm{~nm}$ and $446 \mathrm{~nm}$. Information of the current density versus voltage measurements are provided in Box $\mathbf{3 . 1}$.

\begin{tabular}{c||c|c|c|c}
\hline Thickness $[\mathrm{nm}]$ & $\mathrm{FF}[\%]$ & $J_{\mathrm{sc}}\left[\mathrm{mA} \mathrm{cm}^{2}\right]$ & $V_{\mathrm{oc}}[\mathrm{V}]$ & $\mathrm{PCE}_{\max }\left(\mathrm{PCE}_{\mathrm{avg}}\right)[\%]$ \\
\hline 60 & 68.6 & 15.1 & 0.99 & $10.3(9.9 \pm 0.3)$ \\
90 & 63.0 & 15.0 & 0.98 & $9.3(9.0 \pm 0.2)$ \\
130 & 61.1 & 14.6 & 0.98 & $8.9(8.6 \pm 0.3)$ \\
180 & 55.6 & 15.0 & 0.98 & $8.2(8.0 \pm 0.2)$ \\
288 & 43.8 & 14.5 & 0.97 & $6.2(5.7 \pm 0.3)$ \\
470 & 32.0 & 10.1 & 0.96 & $3.1(2.8 \pm 0.2)$ \\
660 & 29.8 & 4.1 & 0.95 & $1.2(1.0 \pm 0.1)$ \\
\hline
\end{tabular}

Table A.7: Photovoltaic parameters [open-circuit voltage $\left(V_{\mathrm{oc}}\right)$, fill factor $(\mathrm{FF})$, shortcircuit current density $\left(J_{\mathrm{sc}}\right)$, and power-conversion efficiency (PCE)] of PM6:ITIC devices with different active layer thicknesses varying between $60 \mathrm{~nm}$ and $660 \mathrm{~nm}$. Information of the current density versus voltage measurements are provided in Box $\mathbf{3 . 1}$. 
A.4 Device statistics and simulation model parameters

\begin{tabular}{c||c|c|c|c}
\hline Thickness $[\mathrm{nm}]$ & $\mathrm{FF}[\%]$ & $J_{\mathrm{sc}}\left[\mathrm{mA} \mathrm{cm}{ }^{2}\right]$ & $V_{\mathrm{oc}}[\mathrm{V}]$ & $\mathrm{PCE}_{\max }\left(\mathrm{PCE}_{\mathrm{avg}}\right)[\%]$ \\
\hline 50 & 59.8 & 9.1 & 0.94 & $5.1(4.8 \pm 0.3)$ \\
90 & 55.5 & 10.4 & 0.96 & $5.6(5.3 \pm 0.3)$ \\
170 & 44.3 & 10.0 & 0.92 & $4.1(3.8 \pm 0.3)$ \\
200 & 42.3 & 10.1 & 0.91 & $3.9(3.6 \pm 0.3)$ \\
270 & 34.8 & 8.8 & 0.92 & $2.8(2.9 \pm 0.2)$ \\
310 & 31.7 & 7.6 & 0.88 & $2.1(1.9 \pm 0.2)$ \\
\hline
\end{tabular}

Table A.8: Photovoltaic parameters [open-circuit voltage $\left(V_{\mathrm{oc}}\right)$, fill factor $(\mathrm{FF})$, shortcircuit current density $\left(J_{\mathrm{sc}}\right)$, and power-conversion efficiency (PCE)] of PBDB-T:EHIDTBR devices with different active layer thicknesses varying between $50 \mathrm{~nm}$ and 310 nm. Information of the current density versus voltage measurements are provided in Box 3.1. 
A. DEVICE FABRICATION, STATISTICS, AND SIMULATION MODEL PARAMETERS 


\section{Appendix B}

\section{Additional data and figures}

The following part of the appendix contains additional drift-diffusion simulation and experimental data, and additional figures are provided.

This appendix is written based upon collaborative works of the author (i) published in the journals ACS Photonics [see Chapter 2] and Nature Communications [see Chapter 6], (ii) submitted to the journal Energy 83 Environmental Science [see Chapter 3] and Physical Review Applied [see Chapter 5], and (iii) currently under preparation for submission [see Chapter 4]. 


\section{B. ADDITIONAL DATA AND FIGURES}

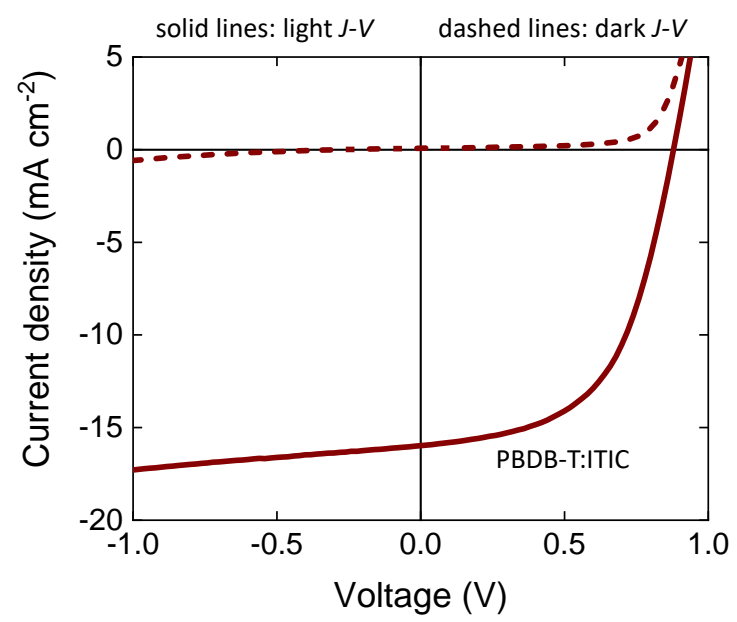

Figure B.1: Current density versus applied voltage $[J-V]$ performance of a $\sim 100 \mathrm{~nm}$ thick PBDB-T:ITIC solar cell measured in dark [dashed line] and under artificial 1 sun AM 1.5G illumination [solid line]. Detailed information of the $J$ - $V$ measurement technique are provided in Box 3.1.

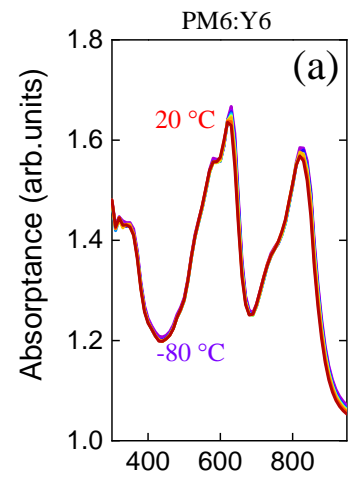

Wavelength $(\mathrm{nm})$
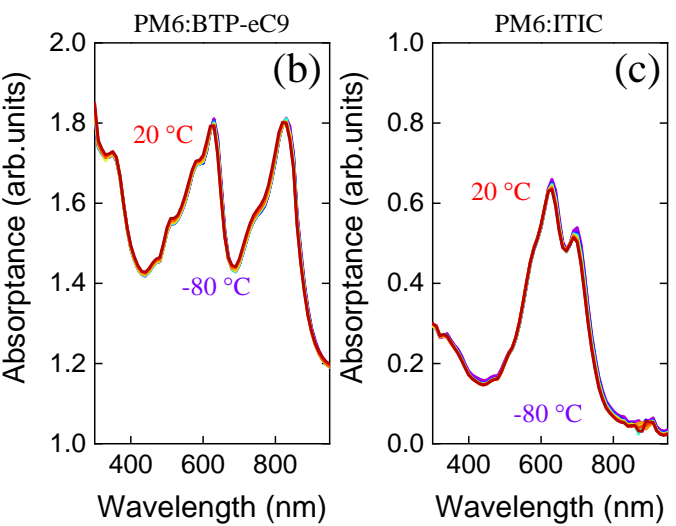

Figure B.2: Temperature dependent absorptance spectra of (a) PM6:Y6, (b) PM6:BTPeC9, (c) PM6:ITIC, and (d) PBDB-T:EH-IDTBR plotted as a function of wavelength, and compared for different temperatures. The temperatures were stepwise varied between $20{ }^{\circ} \mathrm{C}$ and $-80^{\circ} \mathrm{C}$. Details of the temperature dependent absorptance measurements are provided in Box 3.2 . 

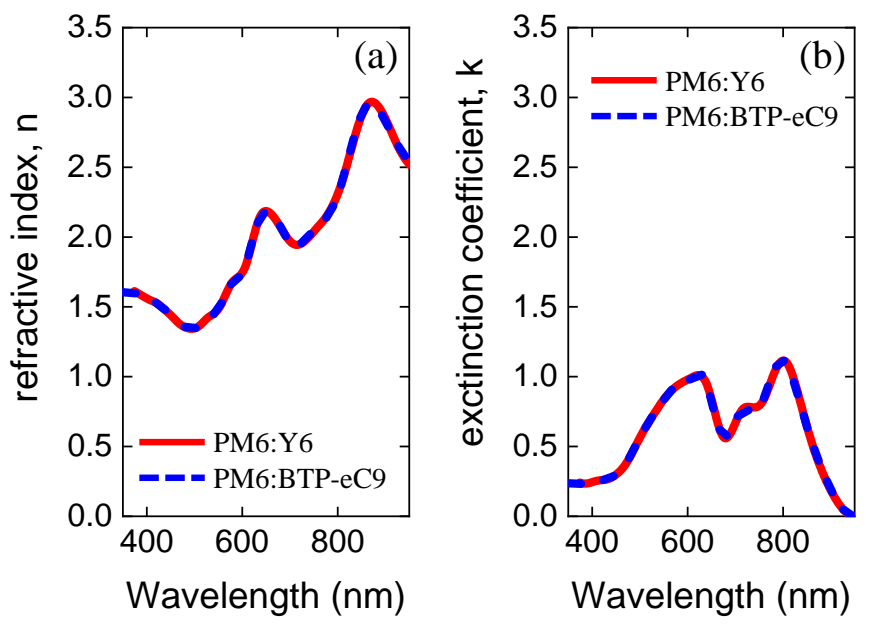

Figure B.3: (a) Refractive index $n$ of PM6:Y6 [red] and PM6:BTP-eC9 [blue] plotted as a function of wavelength. (b) Extinction coefficient $k$ of PM6:Y6 [red] and PM6:BTPeC9 [blue] plotted as a function of wavelength. Refractive and extinction coefficients were obtained via ellipsometry - details of the measurement technique are provided in [102].
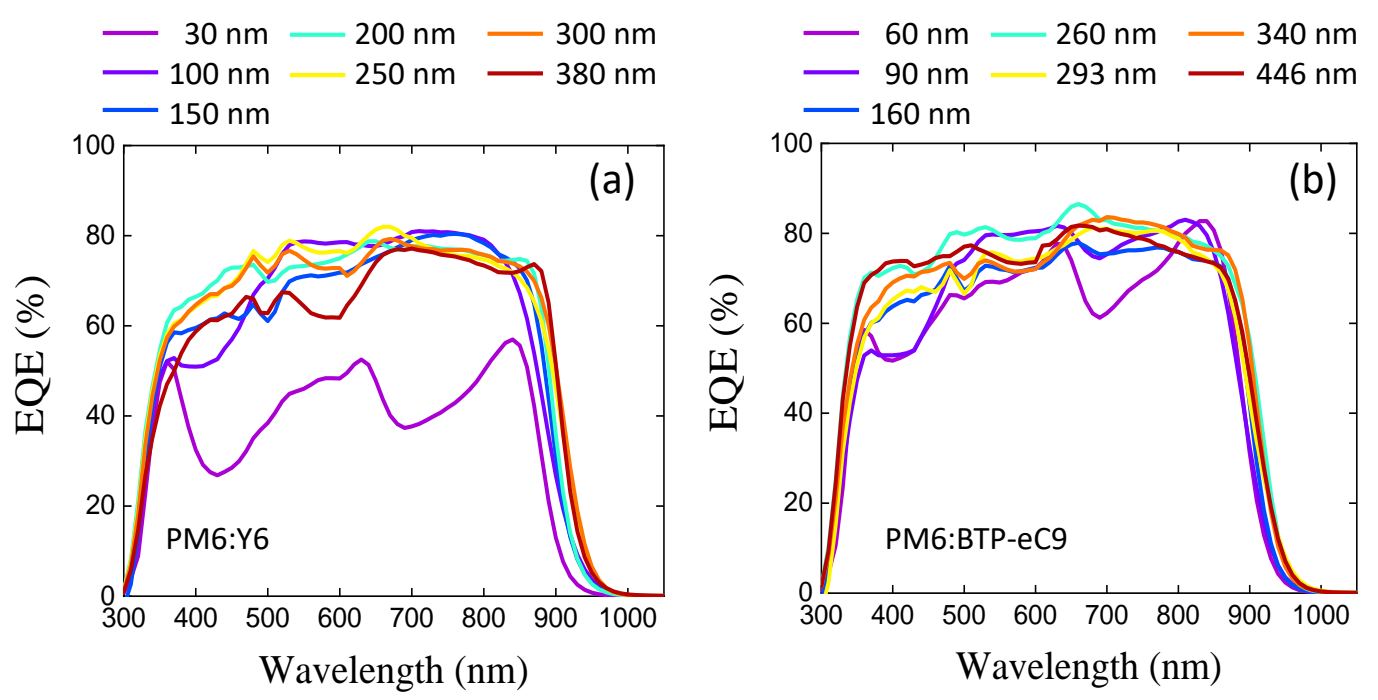

Figure B.4: External quantum efficiency [EQE] spectra of (a) PM6:Y6 and (b) PM6:BTP-eC9 plotted as a function of wavelength, and compared for different active layer thicknesses. Detailed information of the EQE measurement technique are provide in Chapter 2. 

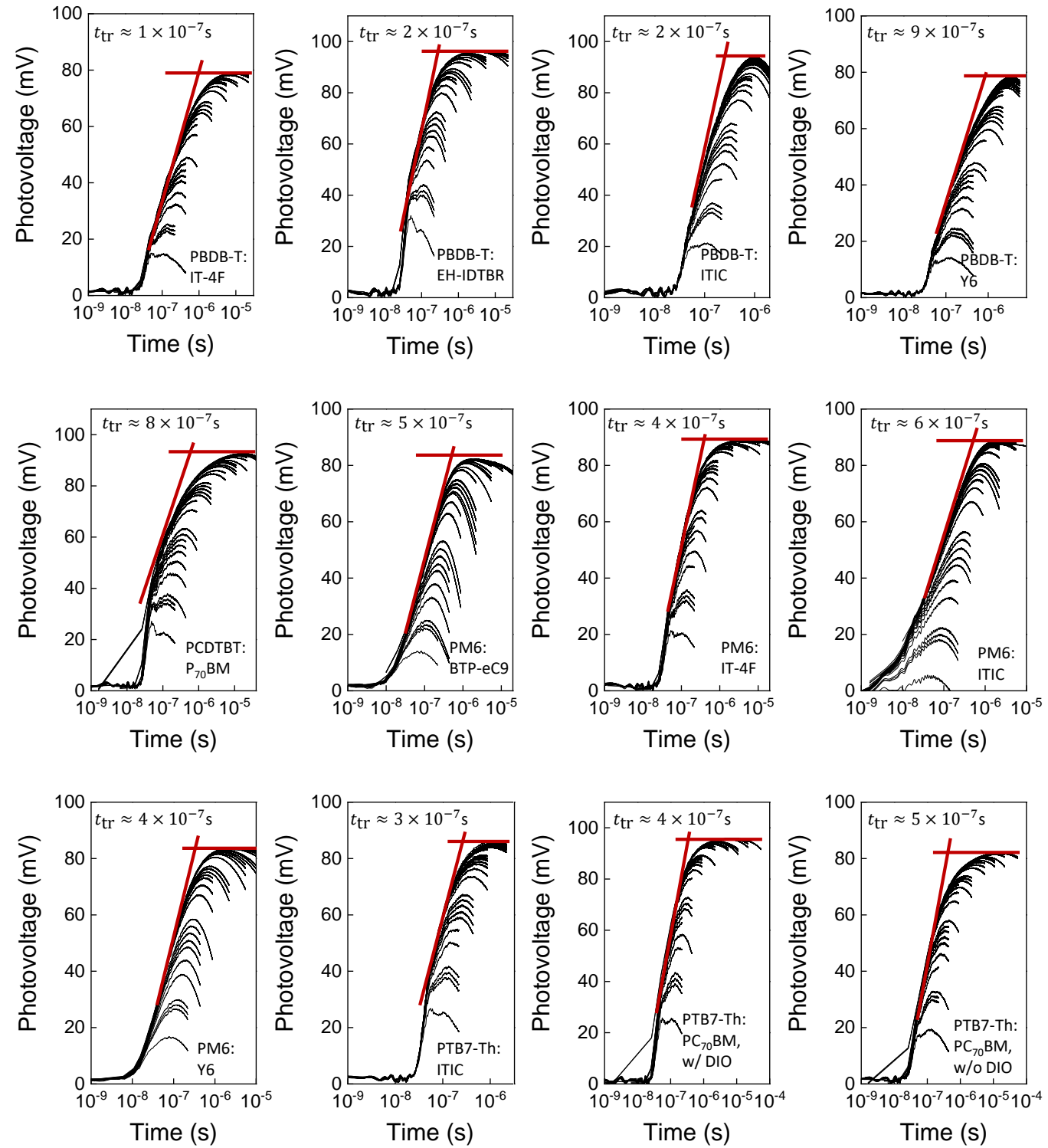

Figure B.5: Resistance-dependent photovoltage $[\mathrm{RPV}]$ transient signals under shortcircuit condition measured on a large variety of fullerene and non-fullerene acceptor based organic solar cells. The load resistance was stepwise varied between $50 \Omega$ and $1 \mathrm{M} \Omega$ changing the magnitude of the transient signals. The mobilities $\mu$ were calculated based upon the transit time $t_{\mathrm{tr}}$ via $\mu=d^{2}\left[V_{\mathrm{bi}} t_{\mathrm{tr}}\right]^{-1}$, where $d$ is the thickness of the corresponding active layer [see Appendix $\mathbf{A}$ and $V_{\mathrm{bi}}$ denotes the built-in voltage. Detailed information of the RPV measurement technique are provided in Box 3.3 . 


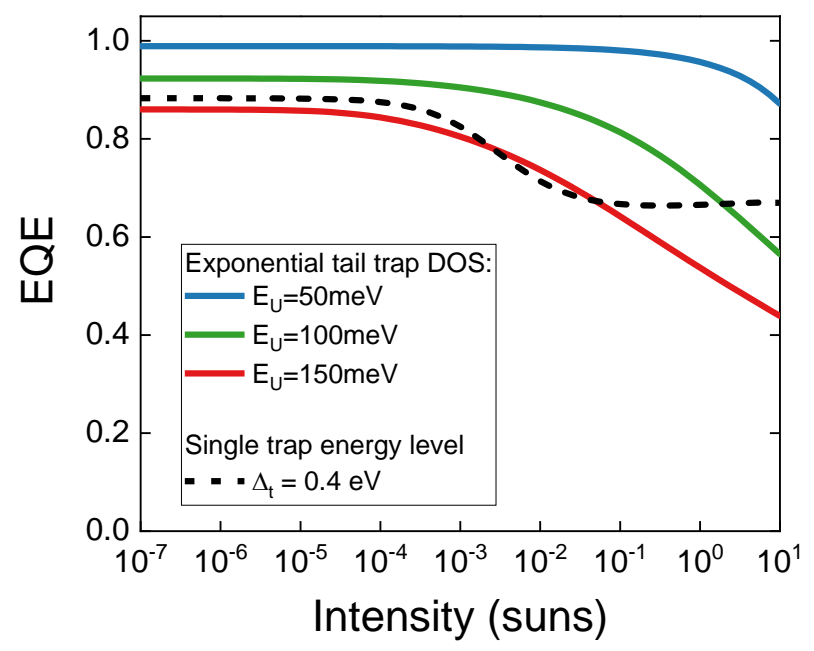

Figure B.6: Simulated external quantum efficiency [EQE] for the case of exponential tail trap density of states [DOS] plotted as a function of intensity, and compared for different Urbach energy values [solid lines]. For comparison, a case of a single trap energy level with $\Delta_{\mathrm{t}}=0.4 \mathrm{eV}$ [dashed line] is included.

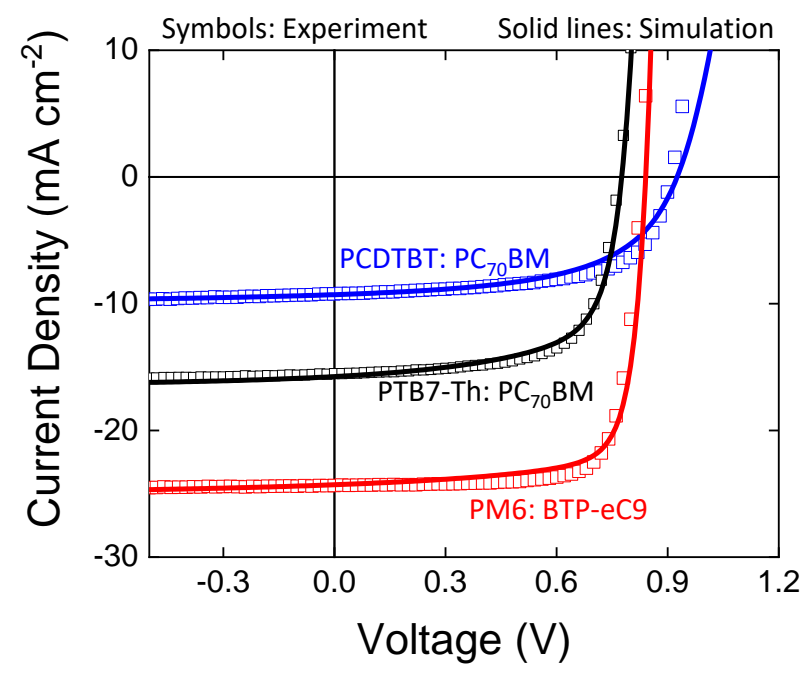

Figure B.7: Experimentally obtained [symbols] and simulated [solid lines] current density versus applied voltage curves of $\mathrm{PCDTBT}: \mathrm{PC}_{70} \mathrm{BM}$ [blue], PTB7-Th:PC ${ }_{70} 0 \mathrm{BM}$ [black] and PM6:BTP-eC9 [red] under artificial 1 sun AM 1.5G conditions. No hysteresis or dependence on the scan speed was observed. Experimental information of the $J$ - $V$ measurement technique are provided in Box 3.1; simulation model parameters are provided in Tab. A.3. 


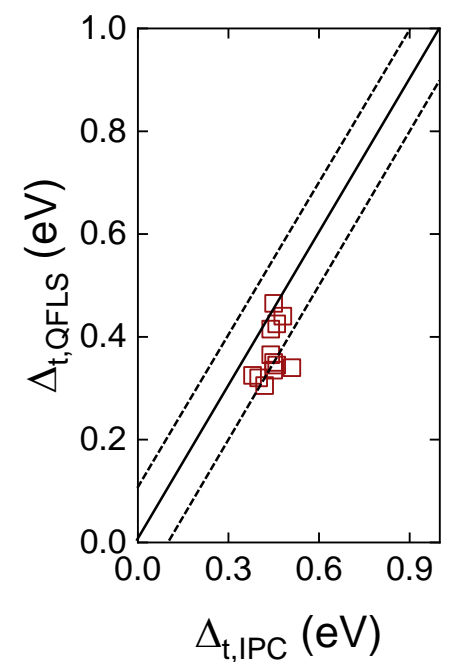

Figure B.8: Comparison between trap depths estimated from IPC $\left[\Delta_{t, I P C}\right]$ as described in Chapter 5, Section 5.6.1 and via quasi-Fermi level splitting at the point-of-transition $\left[\Delta_{\mathrm{t}, \mathrm{QFLS}}\right]$ obtained from the open-circuit voltage [see Chapter 5, Section 5.6.2] . For comparison, $\Delta_{\mathrm{t}, \mathrm{QFLS}}=\Delta_{\mathrm{t}, \mathrm{IPC}}\left[\Delta_{\mathrm{t}, \mathrm{QFLS}}=\Delta_{\mathrm{t}, \mathrm{IPC}} \pm 0.1 \mathrm{eV}\right]$ is indicated by the black solid line [dashed lines]. 


\section{Bibliography}

[1] John Cleland. World Population Growth; Past, Present and Future. Environmental and Resource Economics, 55(4):543-554, 2013. 2

[2] Population Division United Nations, Department of Economic and Social Affairs. World Population Prospects 2019 - Highlights. Technical report, 2019. 2

[3] IEA. IEA World Energy Outlook Report. Technical Report 6, 2010. 2

[4] IEA. "World Energy Outlook 2019", IEA, Paris. Technical report, 2019. 2

[5] United Nations Environment Programme. Emissions Gap Report 2020. Technical report, 2020. 2

[6] M. Oppenheimer, B.C. Glavovic, J. Hinkel, R. van de Wal, A.K. Magnan, A. Abd-Elgawad, R. Cai, M. Cifuentes-Jara, R.M. DeConto, T. Ghosh, J. Hay, F. Isla, B. Marzeion, B. Meyssignac, and Z. Sebesvari. Sea Level Rise and Implications for Low-Lying Islands, Coasts and Communities. Technical report, 2019.

[7] R. Hock, G. Rasul, C. Adler, B. Cáceres, S. Gruber, Y. Hirabayashi, M. Jackson, A. Kääb, S. Kang, S. Kutuzov, A. Milner, U. Molau, S. Morin, B. Orlove, and H. I. Steltzer. Chapter 2: High Mountain Areas. IPCC Special Report on the Ocean and Cryosphere in a Changing Climate. 2019.

[8] Matthew Collins, Michael Sutherland, Laurens Bouwer, So-Min Cheong, Thomas L. Frölicher, Helene Jacot Des Combes, Mathew Koll Roxy, Iñigo Losada, Kathleen L. McInnes, Beate Ratter, Evelia Rivera-Arriaga, Raden Dwi Susanto, Didier Swingedouw, Lourdes Tibig, Pepijn Bakker, C. Mark Eakin, Kerry Emanuel, Michael Grose, Mark Hemer, Laura Jackson, Andreas Kääb, Jules B. Kajtar, Thomas Knutson, Charlotte Laufkötter, Ilan Noy, Mark Payne, Roshanka 


\section{BIBLIOGRAPHY}

Ranasinghe, Giovanni Sgubin, and Mary-Louise Timmermans. Extremes, Abrupt Changes and Managing Risks. 2019. 2

[9] Becquerel A E. Recherches sur les effets de la radiation chimique de la lumiere solaire au moyen des courants electriques. Comptes Rendus de L'Academie des Sciences, 9:145-149, 1839. 2

[10] E. Becquerel. Mémoire sur les effets électriques produits sous l'influence des rayons solaires. Comptes Rendus, 9:561-567, 1839. 2

[11] D. M. Chapin, C. S. Fuller, and G. L. Pearson. A New Silicon p-n Junction Photocell for Converting Solar Radiation into Electrical Power. Journal of Applied Physics, 25(5):676-677, 1954. 2

[12] Kunta Yoshikawa, Hayato Kawasaki, Wataru Yoshida, Toru Irie, Katsunori Konishi, Kunihiro Nakano, Toshihiko Uto, Daisuke Adachi, Masanori Kanematsu, Hisashi Uzu, and Kenji Yamamoto. Silicon heterojunction solar cell with interdigitated back contacts for a photoconversion efficiency over 26\%. Nature Energy, 2(5), 2017. 3, 9

[13] Martin Green, Ewan Dunlop, Jochen Hohl-Ebinger, Masahiro Yoshita, Nikos Kopidakis, and Xiaojing Hao. Solar cell efficiency tables (version 57). Progress in Photovoltaics: Research and Applications, 29(1):3-15, 2021. 3, 9

[14] A. Pochettino. On the photo-electric behavior of the Anthracene. Acad. Lincei Rendiconti, 15(5):355-363, 1906. 3

[15] C. W. Tang and A. C. Albrecht. Photovoltaic effects of metal-chlorophyll-a-metal sandwich cells. The Journal of Chemical Physics, 62:2139-2149, 1975. 3

[16] M Born and R Oppenheimer. Zur Quantentheorie der Molekülen. Annalen der Physik, 20(84):457-484, 1927. 4

[17] W. Pauli. Über den Zusammenhang des Abschlusses der Elektronengruppen im Atom mit der Komplexstruktur der Spektren. Zeitschrift für Physik, 31(1):765783, 1925. 4 
[18] Rudolf Gross and Achim Marx. Festkörperphysik. Oldenbourg Verlag München, 2012. 4, 5, 7, 8, 12

[19] P. Würfel. Physics of Solar Cells: Basic Principles to Advanced Concepts. 2009. 6

[20] J. M. Ziman. Principles of the Theory of Solids. Cambridge University Press, jul 1972. 6

[21] Anna Köhler and Heinz Bässler. Electronic processes in organic semiconductors: An introduction. Wiley-VCH, 2015. 7, 12

[22] Ardalan Armin, Wei Li, Oskar J. Sandberg, Zuo Xiao, Liming Ding, Jenny Nelson, Dieter Neher, Koen Vandewal, Safa Shoaee, Tao Wang, Harald Ade, Thomas Heumüller, Christoph Brabec, and Paul Meredith. A history and perspective of non-fullerene electron acceptors for organic solar cells. Advanced Energy Materials, 11(15), 2021. 13, 15, 16, 22, 27, 60, 61

[23] C W Tang. Two-layer organic photovoltaic cell. Applied Physics Letters, 48(2):183-185, 1986. 14

[24] Masahiro Hiramoto, Hiroshi Fujiwara, and Masaaki Yokoyama. Three-layered organic solar cell with a photoactive interlayer of codeposited pigments. Applied Physics Letters, 58(10):1062-1064, 1991. 14

[25] Shigenori Morita, Anvar A. Zakhidov, and Katsumi Yoshino. Doping effect of buckminsterfullerene in conducting polymer: Change of absorption spectrum and quenching of luminescene. Solid State Communications, 82(4):249-252, 1992. 14

[26] Paul W.M. Blom, Valentin D. Mihailetchi, L. Jan Anton Koster, and Denis E. Markov. Device physics of polymer:Fullerene bulk heterojunction solar cells. Advanced Materials, 19(12):1551-1566, 2007. 14, 17

[27] Tracey M. Clarke and James R. Durrant. Charge photogeneration in organic solar cells. Chemical Reviews, 110(11):6736-6767, 2010. 14, 24, 80, 96

[28] Simon M. Sze and Kwok K. Ng. Physics of Semiconductor Devices. Wiley \& Sons, 3rd edition, 1981. 15 


\section{BIBLIOGRAPHY}

[29] Ardalan Armin, Ivan Kassal, Paul E. Shaw, Mike Hambsch, Martin Stolterfoht, Dani M. Lyons, Jun Li, Zugui Shi, Paul L. Burn, and Paul Meredith. Spectral dependence of the internal quantum efficiency of organic solar cells: Effect of charge generation pathways. Journal of the American Chemical Society, 136(32):1146511472, 2014. 15, 81

[30] Dani M. Stoltzfus, Jenny E. Donaghey, Ardalan Armin, Paul E. Shaw, Paul L. Burn, and Paul Meredith. Charge Generation Pathways in Organic Solar Cells: Assessing the Contribution from the Electron Acceptor. Chemical Reviews, 116(21):12920-12955, 2016. 15

[31] Gilles Dennler, Markus C. Scharber, and Christoph J. Brabec. Polymer-fullerene bulk-heterojunction solar cells. Advanced Materials, 21(13):1323-1338, 2009. 15

[32] L. Jan Anton Koster, Sean E. Shaheen, and Jan C. Hummelen. Pathways to a new effi ciency regime for organic solar cells. Advanced Energy Materials, 2(10):12461253, 2012. 15

[33] Lorena Perdigón-Toro, Huotian Zhang, Anastasia Markina, Jun Yuan, Seyed Mehrdad Hosseini, Christian M. Wolff, Guangzheng Zuo, Martin Stolterfoht, Yingping Zou, Feng Gao, Denis Andrienko, Safa Shoaee, and Dieter Neher. Barrierless Free Charge Generation in the High-Performance PM6:Y6 Bulk Heterojunction Non-Fullerene Solar Cell. Advanced Materials, 32(9):1906763, 2020. 15, 27, 61, 62

[34] Paul E. Shaw, Arvydas Ruseckas, and I. D W Samuel. Exciton diffusion measurements in poly(3-hexylthiophene). Advanced Materials, 20(18):3516-3520, 2008. 17

[35] Oleksandr V. Mikhnenko, Hamed Azimi, Markus Scharber, Mauro Morana, Paul W.M. Blom, and Maria Antonietta Loi. Exciton diffusion length in narrow bandgap polymers. Energy and Environmental Science, 5(5):6960-6965, 2012.

[36] Nasim Zarrabi, Aren Yazmaciyan, Paul Meredith, Ivan Kassal, and Ardalan Armin. Anomalous Exciton Quenching in Organic Semiconductors in the LowYield Limit. Journal of Physical Chemistry Letters, 9(20):6144-6148, 2018. 17 
[37] Johannes Benduhn, Kristofer Tvingstedt, Fortunato Piersimoni, Sascha Ullbrich, Yeli Fan, Manuel Tropiano, Kathryn A. McGarry, Olaf Zeika, Moritz K. Riede, Christopher J. Douglas, Stephen Barlow, Seth R. Marder, Dieter Neher, Donato Spoltore, and Koen Vandewal. Intrinsic non-radiative voltage losses in fullerenebased organic solar cells. Nature Energy, 2(6):17053, 2017. 17, 60

[38] Ester Buchaca-Domingo, Koen Vandewal, Zhuping Fei, Scott E. Watkins, Fiona H. Scholes, James H. Bannock, John C. De Mello, Lee J. Richter, Dean M. DeLongchamp, Aram Amassian, Martin Heeney, Alberto Salleo, and Natalie Stingelin. Direct correlation of charge transfer absorption with molecular donor:acceptor interfacial area via photothermal deflection spectroscopy. Journal of the American Chemical Society, 137(16):5256-5259, 2015. 17

[39] Ye Huang, Edward J. Kramer, Alan J. Heeger, and Guillermo C. Bazan. Bulk heterojunction solar cells: Morphology and performance relationships. Chemical Reviews, 114(14):7006-7043, 2014. 17

[40] Mathias Nyman, Oskar J. Sandberg, Wei Li, Stefan Zeiske, Robin Kerremans, Paul Meredith, and Ardalan Armin. Requirements for Making Thick Junctions of Organic Solar Cells based on Nonfullerene Acceptors. Solar RRL, 5(5):2100018, 2021. 17, 75

[41] Ardalan Armin, Marappan Velusamy, Pascal Wolfer, Yuliang Zhang, Paul L. Burn, Paul Meredith, and Almantas Pivrikas. Quantum efficiency of organic solar cells: Electro-optical cavity considerations. ACS Photonics, 1(3):173-181, 2014. 18, 81

[42] Christina Kaiser, Stefan Zeiske, Paul Meredith, and Ardalan Armin. Determining Ultralow Absorption Coefficients of Organic Semiconductors from the SubBandgap Photovoltaic External Quantum Efficiency. Advanced Optical Materials, $8(1): 1-7,2020$. 55, 57, 132, 134

[43] Ardalan Armin, Nasim Zarrabi, Oskar J. Sandberg, Christina Kaiser, Stefan Zeiske, Wei Li, and Paul Meredith. Limitations of Charge Transfer State Parameterization Using Photovoltaic External Quantum Efficiency. Advanced Energy Materials, 10(41):1-10, 2020. 18, 56, 57 


\section{BIBLIOGRAPHY}

[44] W Shockley. The Theory of p-n Junctions in Semiconductors and p-n Junction Transistors. Bell System Technical Journal, 28(3):435-489, 1949. 19

[45] Christoph Waldauf, Marcus C Scharber, Pavel Schilinsky, Jens A Hauch, and Christoph J Brabec. Physics of organic bulk heterojunction devices for photovoltaic applications. Journal of Applied Physics, 99(10):104503, 2006. 19

[46] Carsten Deibel and Vladimir Dyakonov. Polymer-fullerene bulk heterojunction solar cells. Reports on Progress in Physics, 73(9):96401-96439, 2010. 19, 20, 92, 96

[47] Thomas Kirchartz, Florent Deledalle, Pabitra Shakya Tuladhar, James R. Durrant, and Jenny Nelson. On the differences between dark and light ideality factor in polymer:Fullerene solar cells. Journal of Physical Chemistry Letters, 4(14):2371-2376, 2013. 19, 97

[48] Kristofer Tvingstedt and Carsten Deibel. Temperature Dependence of Ideality Factors in Organic Solar Cells and the Relation to Radiative Efficiency. Advanced Energy Materials, 6(9):1502230, 2016. 97, 123

[49] Nasim Zarrabi, Oskar J. Sandberg, Stefan Zeiske, Wei Li, Drew B. Riley, Paul Meredith, and Ardalan Armin. Charge-generating mid-gap trap states define the thermodynamic limit of organic photovoltaic devices. Nature Communications, 11:5567, 2020. 19, 24, 55, 57, 73, 132

[50] Koen Vandewal, Kristofer Tvingstedt, Abay Gadisa, Olle Inganäs, and Jean V. Manca. On the origin of the open-circuit voltage of polymer-fullerene solar cells. Nature Materials, 8(11):904-909, 2009. 20, 40

[51] Koen Vandewal. Interfacial charge transfer states in condensed phase systems. Annual Review of Physical Chemistry, 67:113-133, 2016. 20, 24, 25

[52] Louise C Hirst and Nicholas J Ekins-Daukes. Fundamental losses in solar cells. Progress in Photovoltaics: Research and Applications, 19(3):286-293, 2011. 21, 22

[53] William Shockley and Hans J Queisser. Detailed balance limit of efficiency of p-n junction solar cells. Journal of Applied Physics, 32(3):510-519, 1961. 21 
[54] Mohammed Azzouzi, Jun Yan, Thomas Kirchartz, Kaikai Liu, Jinliang Wang, Hongbin Wu, and Jenny Nelson. Nonradiative Energy Losses in BulkHeterojunction Organic Photovoltaics. Physical Review X, 8(3):31055, 2018. 22, 40

[55] Mumin Shi, Tao Wang, Yao Wu, Rui Sun, Wei Wang, Jing Guo, Qiang Wu, Wenyan Yang, and Jie Min. The Intrinsic Role of Molecular Mass and Polydispersity Index in High-Performance Non-Fullerene Polymer Solar Cells. Advanced Energy Materials, 11(1), 2021. 22

[56] Drew B. Riley, Oskar J. Sandberg, Nora M. Wilson, Wei Li, Stefan Zeiske, Nasim Zarrabi, Paul Meredith, Ronald Österbacka, and Ardalan Armin. Direct Quantification of Quasi-Fermi-Level Splitting in Organic Semiconductor Devices. Physical Review Applied, 15(6):064035, 2021. 22

[57] Wei Li, Mengxue Chen, Jinlong Cai, Emma L.K. Spooner, Huijun Zhang, Robert S. Gurney, Dan Liu, Zuo Xiao, David G. Lidzey, Liming Ding, and Tao Wang. Molecular Order Control of Non-fullerene Acceptors for High-Efficiency Polymer Solar Cells. Joule, 3(3):819-833, 2019. 23

[58] Sheridan Few, Jarvist M. Frost, and Jenny Nelson. Models of charge pair generation in organic solar cells. Physical Chemistry Chemical Physics, 17(4):2311-2325, 2015. 24

[59] Kristofer Tvingstedt, Koen Vandewal, Abay Gadisa, and Fengling Zhang. Electroluminescence from Charge Transfer States in Polymer. Journal of the American Chemical Society, 10(3):11819-11824, 2009. 24

[60] Koen Vandewal, Kristofer Tvingstedt, Abay Gadisa, Olle Inganäs, and Jean V. Manca. Relating the open-circuit voltage to interface molecular properties of donor:acceptor bulk heterojunction solar cells. Physical Review B - Condensed Matter and Materials Physics, 81(12):1-8, 2010. 24, 40, 136

[61] Koen Vandewal, Steve Albrecht, Eric T. Hoke, Kenneth R. Graham, Johannes Widmer, Jessica D. Douglas, Marcel Schubert, William R. Mateker, Jason T. Bloking, George F. Burkhard, Alan Sellinger, Jean M.J. J. Fréchet, Aram Amassian, Moritz K. Riede, Michael D. McGehee, Dieter Neher, and Alberto Salleo. 


\section{BIBLIOGRAPHY}

Efficient charge generation by relaxed charge-transfer states at organic interfaces. Nature Materials, 13(1):63-68, 2014. 25, 27, 40, 80

[62] Jun Yan, Elham Rezasoltani, Mohammed Azzouzi, Flurin Eisner, and Jenny Nelson. Influence of static disorder of charge transfer state on voltage loss in organic photovoltaics. Nature Communications, 12(1):3642, 2021. 25

[63] M. Knupfer. Exciton binding energies in organic semiconductors. Applied Physics A: Materials Science and Processing, 77(5):623-626, 2003. 26

[64] R. A. Marcus. On the theory of oxidation-reduction reactions involving electron transfer. I. The Journal of Chemical Physics, 24(5):966-978, 1956. 26

[65] Maria Saladina, Pablo Simón Marqués, Anastasia Markina, Safakath Karuthedath, Christopher Wöpke, Clemens Göhler, Yue Chen, Magali Allain, Philippe Blanchard, Clément Cabanetos, Denis Andrienko, Frédéric Laquai, Julien Gorenflot, and Carsten Deibel. Charge Photogeneration in Non-Fullerene Organic Solar Cells: Influence of Excess Energy and Electrostatic Interactions. Advanced Functional Materials, 31(8):2007479, 2021. 27

[66] Jona Kurpiers, Thomas Ferron, Steffen Roland, Marius Jakoby, Tobias Thiede, Frank Jaiser, Steve Albrecht, Silvia Janietz, Brian A. Collins, Ian A. Howard, and Dieter Neher. Probing the pathways of free charge generation in organic bulk heterojunction solar cells. Nature Communications, 9(1), 2018. 27, 40, 80, 81

[67] L. Onsager. Initial recombination of ions. Physical Review, 54(8):554-557, 1938. 27

[68] Charles L. Braun. Electric field assisted dissociation of charge transfer states as a mechanism of photocarrier production. The Journal of Chemical Physics, 80(9):4157-4161, 1984. 27, 28, 80

[69] Allen Miller and Elihu Abrahams. Impurity conduction at low concentrations. Physical Review, 120(3):745-755, 1960. 30 
[70] A. Laudari and S. Guha. Temperature dependent carrier mobility in organic field-effect transistors: The role of dielectrics. Journal of Applied Physics, 125(3):35501, 2019. 30

[71] M. Langevin. Recombinaison et mobilites des ions dans les gaz. Annales de Chimie et de Physique, 28(122):433-530, 1903. 32

[72] M. Langevin. Recombinaison et diffusion des ions gazeux. Journal de Physique Théorique et Appliquée, 4(1):322-333, 1905. 32

[73] Seyed Mehrdad Hosseini, Steffen Roland, Jona Kurpiers, Zhiming Chen, Kai Zhang, Fei Huang, Ardalan Armin, Dieter Neher, and Safa Shoaee. Impact of Bimolecular Recombination on the Fill Factor of Fullerene and NonfullereneBased Solar Cells: A Comparative Study of Charge Generation and Extraction. Journal of Physical Chemistry C, 123(11):6823-6830, 2019. 32

[74] Gert-Jan Jan A.H. Wetzelaer, Niels J Van Der Kaap, Jan Anton Koster, and Paul W.M. Blom. Quantifying bimolecular recombination in organic solar cells in steady state. Advanced Energy Materials, 3(9):1130-1134, 2013.

[75] Nasim Zarrabi, Oskar J. Sandberg, Christina Kaiser, Jegadesan Subbiah, David J. Jones, Paul Meredith, and Ardalan Armin. Experimental Evidence Relating Charge-Transfer-State Kinetics and Strongly Reduced Bimolecular Recombination in Organic Solar Cells. The Journal of Physical Chemistry Letters, 11(24):10519-10525, 2020. 32, 133

[76] L. Jan Anton Koster, V. D. Mihailetchi, and P. W.M. Blom. Bimolecular recombination in polymer/fullerene bulk heterojunction solar cells. Applied Physics Letters, 88(5):1-3, 2006. 33

[77] Michael C. Heiber, Christoph Baumbach, Vladimir Dyakonov, and Carsten Deibel. Encounter-limited charge-carrier recombination in phase-separated organic semiconductor blends. Physical Review Letters, 114(13), 2015. 33, 70

[78] W Shockley, W.T. Read, W Shocklev, and Axd W T Read. Statistics of the Recombination of Holes and Electrons. Physical Review, 87(5):835-842, 1952. 34 


\section{BIBLIOGRAPHY}

[79] Murray A. Lampert and Peter Mark. Current injection in solids. Academic Press, 1970. 34, 35, 84

[80] Oskar J. Sandberg, Mathias Nyman, and Ronald Österbacka. Effect of contacts in organic bulk heterojunction solar cells. Physical Review Applied, 1(2):024003, 2014. 37, 73, 87, 99, 124

[81] Oskar J. Sandberg, Anton Sundqvist, Mathias Nyman, and Ronald Österbacka. Relating Charge Transport, Contact Properties, and Recombination to OpenCircuit Voltage in Sandwich-Type Thin-Film Solar Cells. Physical Review Applied, 5(4):044005, 2016. 37, 73, 87, 97, 99, 124

[82] Stefan Zeiske, Christina Kaiser, Paul Meredith, and Ardalan Armin. Sensitivity of Sub-Bandgap External Quantum Efficiency Measurements of Solar Cells under Electrical and Light Bias. ACS Photonics, 7(1):256-264, 2020. 39, 131

[83] Carolin M. Sutter-Fella, D. Westley Miller, Quynh P. Ngo, Ellis T. Roe, Francesca M. Toma, Ian D. Sharp, Mark C. Lonergan, and Ali Javey. Band tailing and deep defect states in $\mathrm{CH} 3 \mathrm{NH} 3 \mathrm{~Pb}(\mathrm{I} 1-\mathrm{xBrx}) 3$ perovskites as revealed by sub-bandgap photocurrent. ACS Energy Letters, 2(3):709-715, 2017. 40

[84] D. Westley Miller, Giles E. Eperon, Ellis T. Roe, Charles W. Warren, Henry J. Snaith, and Mark C. Lonergan. Defect states in perovskite solar cells associated with hysteresis and performance. Applied Physics Letters, 109(15):153902, 2016. 40

[85] Jizhong Yao, Thomas Kirchartz, Michelle S. Vezie, Mark A. Faist, Wei Gong, Zhicai He, Hongbin Wu, Joel Troughton, Trystan Watson, Daniel Bryant, and Jenny Nelson. Quantifying losses in open-circuit voltage in solution-processable solar cells. Physical Review Applied, 4(1):1-10, 2015. 40

[86] S. Matthew Menke, Niva A. Ran, Guillermo C. Bazan, and Richard H. Friend. Understanding Energy Loss in Organic Solar Cells: Toward a New Efficiency Regime. Joule, 2(1):25-35, 2018. 
[87] Samantha Hood, Nasim Zarrabi, Paul Meredith, Ivan Kassal, and Ardalan Armin. Measuring Energetic Disorder in Organic Semiconductors Using the Photogenerated Charge-Separation Efficiency. Journal of Physical Chemistry Letters, 10(14):3863-3870, 2019. 40

[88] Stefano Pisoni, Martin Stolterfoht, Johannes Löckinger, Thierry Moser, Yan Jiang, Pietro Caprioglio, Dieter Neher, Stephan Buecheler, and Ayodhya N. Tiwari. On the origin of open-circuit voltage losses in flexible n-i-p perovskite solar cells. Science and Technology of Advanced Materials, 20(1):786-795, 2019. 40

[89] K. Vandewal, L. Goris, I. Haeldermans, M. Nesládek, K. Haenen, P. Wagner, and J. V. Manca. Fourier-Transform Photocurrent Spectroscopy for a fast and highly sensitive spectral characterization of organic and hybrid solar cells. Thin Solid Films, 516(20):7135-7138, 2008. 40

[90] Shangshang Chen, Yuming Wang, Lin Zhang, Jingbo Zhao, Yuzhong Chen, Danlei Zhu, Huatong Yao, Guangye Zhang, Wei Ma, Richard H. Friend, Philip C.Y. Chow, Feng Gao, and He Yan. Efficient Nonfullerene Organic Solar Cells with Small Driving Forces for Both Hole and Electron Transfer. Advanced Materials, 30(45):1-7, 2018.

[91] Koen Vandewal, Abay Gadisa, Wibren D. Oosterbaan, Sabine Bertho, Fateme Banishoeib, Ineke Van Severen, Laurence Lutsen, Thomas J. Cleij, Dirk Vanderzande, and Jean V. Manca. The relation between open-circuit voltage and the onset of photocurrent generation by charge-transfer absorption in polymer: Fullerene bulk heterojunction solar cells. Advanced Functional Materials, 18(14):2064-2070, 2008. 40

[92] Sascha Ullbrich, Johannes Benduhn, Xiangkun Jia, Vasileios C. Nikolis, Kristofer Tvingstedt, Fortunato Piersimoni, Steffen Roland, Yuan Liu, Jinhan Wu, Axel Fischer, Dieter Neher, Sebastian Reineke, Donato Spoltore, and Koen Vandewal. Emissive and charge-generating donor-acceptor interfaces for organic optoelectronics with low voltage losses. Nature Materials, 18(5):459-464, 2019. 40

[93] Saeed Uz Zaman Khan, Giacomo Londi, Xiao Liu, Michael A. Fusella, Gabriele D’Avino, Luca Muccioli, Alyssa N. Brigeman, Bjoern Niesen, Terry Chien Jen 
Yang, Yoann Olivier, Jordan T. Dull, Noel C. Giebink, David Beljonne, and Barry P. Rand. Multiple Charge Transfer States in Donor-Acceptor Heterojunctions with Large Frontier Orbital Energy Offsets. Chemistry of Materials, 31(17):6808-6817, 2019. 41, 42

[94] F. Pelayo García De Arquer, Ardalan Armin, Paul Meredith, and Edward H. Sargent. Solution-processed semiconductors for next-generation photodetectors. Nature Reviews Materials, 2(3):1-16, 2017.

[95] Fawen Guo, Bin Yang, Yongbo Yuan, Zhengguo Xiao, Qingfeng Dong, Yu Bi, and Jinsong Huang. A nanocomposite ultraviolet photodetector based on interfacial trap-controlled charge injection. Nature Nanotechnology, 7(12):798-802, 2012.

[96] Fawen Guo, Zhengguo Xiao, and Jinsong Huang. Fullerene Photodetectors with a Linear Dynamic Range of 90 dB Enabled by a Cross-Linkable Buffer Layer. Advanced Optical Materials, 1(4):289-294, 2013. 42, 122

[97] Yanjun Fang, Ardalan Armin, Paul Meredith, and Jinsong Huang. Accurate characterization of next-generation thin-film photodetectors. Nature Photonics, 13(1):1-4, 2019. 43

[98] J. Melskens, M. Schouten, R. Santbergen, M. Fischer, R. Vasudevan, D. J. Van Der Vlies, R. J.V. Quax, S. G.M. Heirman, K. Jäger, V. Demontis, M. Zeman, and A. H.M. Smets. In situ manipulation of the sub gap states in hydrogenated amorphous silicon monitored by advanced application of Fourier transform photocurrent spectroscopy. Solar Energy Materials and Solar Cells, 129:70-81, 2014. 55

[99] Mengmeng Hao, Yang Bai, Stefan Zeiske, Long Ren, Junxian Liu, Yongbo Yuan, Nasim Zarrabi, Ningyan Cheng, Mehri Ghasemi, Peng Chen, Miaoqiang Lyu, Dongxu He, Jung Ho Yun, Yi Du, Yun Wang, Shanshan Ding, Ardalan Armin, Paul Meredith, Gang Liu, Hui Ming Cheng, and Lianzhou Wang. Ligand-assisted cation-exchange engineering for high-efficiency colloidal Cs1-xFAxPbI3 quantum dot solar cells with reduced phase segregation. Nature Energy, 5(1):79-88, 2020. 56, 57 
[100] Oskar J. Sandberg, Stefan Zeiske, Nasim Zarrabi, Paul Meredith, and Ardalan Armin. Charge Carrier Transport and Generation via Trap-Mediated Optical Release in Organic Semiconductor Devices. Physical Review Letters, 124(12):128001, 2020. 56, 131

[101] Lorena Perdigón-Toro, Le Quang Phuong, Stefan Zeiske, Koen Vandewal, Ardalan Armin, Safa Shoaee, and Dieter Neher. Excitons Dominate the Emission from PM6:Y6 Solar Cells, but This Does Not Help the Open-Circuit Voltage of the Device. ACS Energy Letters, 6(2):557-564, 2021. 56, 57, 60

[102] Wei Li, Stefan Zeiske, Oskar Sandberg, Drew Riley, Paul Meredith, and Ardalan Armin. Near-unity Charge Generation Yield towards high performance thickjunction Organic Solar Cells. submitted to Energy Environmental Science, 2021. 59, 69, 163

[103] Ye Xu, Huifeng Yao, Lijiao Ma, Jingwen Wang, and Jianhui Hou. Efficient charge generation at low energy losses in organic solar cells: A key issues review. Reports on Progress in Physics, 83(8):082601, 2020. 60

[104] Paul Meredith, Wei Li, and Ardalan Armin. Nonfullerene Acceptors: A Renaissance in Organic Photovoltaics? Advanced Energy Materials, 10(33):2001788, 2020. 60

[105] Timothy M. Burke, Sean Sweetnam, Koen Vandewal, and Michael D. McGehee. Beyond Langevin recombination: How equilibrium between free carriers and charge transfer states determines the open-circuit voltage of organic solar Cells. Advanced Energy Materials, 5(11):1-12, 2015. 60

[106] Markus C. Scharber, David Mühlbacher, Markus Koppe, Patrick Denk, Christoph Waldauf, Alan J. Heeger, and Christoph J. Brabec. Design rules for donors in bulk-heterojunction solar cells - Towards $10 \%$ energy-conversion efficiency. Advanced Materials, 18(6):789-794, 2006. 60

[107] Deping Qian, Zilong Zheng, Huifeng Yao, Wolfgang Tress, Thomas R. Hopper, Shula Chen, Sunsun Li, Jing Liu, Shangshang Chen, Jiangbin Zhang, Xiao Ke Liu, Bowei Gao, Liangqi Ouyang, Yingzhi Jin, Galia Pozina, Irina A. Buyanova, Weimin M. Chen, Olle Inganäs, Veaceslav Coropceanu, Jean Luc Bredas, He Yan, 
Jianhui Hou, Fengling Zhang, Artem A. Bakulin, and Feng Gao. Design rules for minimizing voltage losses in high-efficiency organic solar cells. Nature Materials, 17(8):703-709, 2018. 60

[108] Jun Yuan, Yunqiang Zhang, Liuyang Zhou, Guichuan Zhang, Hin Lap Yip, Tsz Ki Lau, Xinhui Lu, Can Zhu, Hongjian Peng, Paul A. Johnson, Mario Leclerc, Yong Cao, Jacek Ulanski, Yongfang Li, and Yingping Zou. Single-Junction Organic Solar Cell with over 15\% Efficiency Using Fused-Ring Acceptor with ElectronDeficient Core. Joule, 3(4):1140-1151, 2019. 60, 63, 64, 156

[109] Yuan Xie, Weiping Wang, Wei Huang, Fengyuan Lin, Tengfei Li, Sha Liu, Xiaowei Zhan, Yongye Liang, Chao Gao, Hongbin Wu, and Yong Cao. Assessing the energy offset at the electron donor/acceptor interface in organic solar cells through radiative efficiency measurements. Energy and Environmental Science, 12(12):3556-3566, 2019. 60

[110] Huiting Fu, Yuming Wang, Dong Meng, Zetong Ma, Yan Li, Feng Gao, Zhaohui Wang, and Yanming Sun. Suppression of recombination energy losses by decreasing the energetic offsets in perylene diimide-based nonfullerene organic solar cells. ACS Energy Letters, 3(11):2729-2735, 2018. 61

[111] Qishi Liu, Yufan Jiang, Ke Jin, Jianqiang Qin, Jingui Xu, Wenting Li, Ji Xiong, Jinfeng Liu, Zuo Xiao, Kuan Sun, Shangfeng Yang, Xiaotao Zhang, and Liming Ding. 18\% Efficiency organic solar cells. Science Bulletin, 65(4):272-275, 2020. 61, 122

[112] Yong Cui, Huifeng Yao, Jianqi Zhang, Kaihu Xian, Tao Zhang, Ling Hong, Yuming Wang, Ye Xu, Kangqiao Ma, Cunbin An, Chang He, Zhixiang Wei, Feng Gao, and Jianhui Hou. Single-Junction Organic Photovoltaic Cells with Approaching 18\% Efficiency. Advanced Materials, 32(19):1-7, 2020. 63

[113] Yuanbao Lin, Mohamad Insan Nugraha, Yuliar Firdaus, Alberto D. Scaccabarozzi, Filip Aniés, Abdul Hamid Emwas, Emre Yengel, Xiaopeng Zheng, Jiakai Liu, Wandi Wahyudi, Emre Yarali, Hendrik Faber, Osman M. Bakr, 
Leonidas Tsetseris, Martin Heeney, and Thomas D. Anthopoulos. A Simple nDopant Derived from Diquat Boosts the Efficiency of Organic Solar Cells to 18.3\%. ACS Energy Letters, 5(12):3663-3671, 2020. 61

[114] Akchheta Karki, Joachim Vollbrecht, Alexander J. Gillett, Steven Shuyong Xiao, Yali Yang, Zhengxing Peng, Nora Schopp, Alana L. Dixon, Sangcheol Yoon, Max Schrock, Harald Ade, G. N.Manjunatha Reddy, Richard H. Friend, and Thuc Quyen Nguyen. The role of bulk and interfacial morphology in charge generation, recombination, and extraction in non-fullerene acceptor organic solar cells. Energy and Environmental Science, 13(10):3679-3692, 2020. 61, 62, 64

[115] Haixia Zhong, Mahdi Ghorbani-Asl, Khoa Hoang Ly, Jichao Zhang, Jin Ge, Mingchao Wang, Zhongquan Liao, Denys Makarov, Ehrenfried Zschech, Eike Brunner, Inez M. Weidinger, Jian Zhang, Arkady V. Krasheninnikov, Stefan Kaskel, Renhao Dong, and Xinliang Feng. Synergistic electroreduction of carbon dioxide to carbon monoxide on bimetallic layered conjugated metal-organic frameworks. Nature Communications, 11(1):1409, 2020.

[116] Guichuan Zhang, Xian Kai Chen, Jingyang Xiao, Philip C.Y. Chow, Minrun Ren, Grit Kupgan, Xuechen Jiao, Christopher C.S. Chan, Xiaoyan Du, Ruoxi Xia, Ziming Chen, Jun Yuan, Yunqiang Zhang, Shoufeng Zhang, Yidan Liu, Yingping Zou, He Yan, Kam Sing Wong, Veaceslav Coropceanu, Ning Li, Christoph J. Brabec, Jean Luc Bredas, Hin Lap Yip, and Yong Cao. Delocalization of exciton and electron wavefunction in non-fullerene acceptor molecules enables efficient organic solar cells. Nature Communications, 11(1):3943, 2020. 61

[117] Xixiang Zhu, Guichuan Zhang, Jia Zhang, Hin Lap Yip, and Bin Hu. SelfStimulated Dissociation in Non-Fullerene Organic Bulk-Heterojunction Solar Cells. Joule, 4(11):2443-2457, 2020. 61

[118] Ture F. Hinrichsen, Christopher C.S. Chan, Chao Ma, David Paleček, Alexander Gillett, Shangshang Chen, Xinhui Zou, Guichuan Zhang, Hin Lap Yip, Kam Sing Wong, Richard H. Friend, He Yan, Akshay Rao, and Philip C.Y. Chow. Longlived and disorder-free charge transfer states enable endothermic charge separation in efficient non-fullerene organic solar cells. Nature Communications, 11(1), 2020. 61, 62, 80 


\section{BIBLIOGRAPHY}

[119] Kyra N. Schwarz, Paul B. Geraghty, Valerie D. Mitchell, Saeed Uz Zaman Khan, Oskar J. Sandberg, Nasim Zarrabi, Bryan Kudisch, Jegadesan Subbiah, Trevor A. Smith, Barry P. Rand, Ardalan Armin, Gregory D. Scholes, David J. Jones, and Kenneth P. Ghiggino. Reduced Recombination and Capacitor-like Charge Buildup in an Organic Heterojunction. Journal of the American Chemical Society, 142(5):2562-2571, 2020. 61, 69

[120] Yuhang Liu, Jingbo Zhao, Zhengke Li, Cheng Mu, Wei Ma, Huawei Hu, Kui Jiang, Haoran Lin, Harald Ade, and He Yan. Aggregation and morphology control enables multiple cases of high-efficiency polymer solar cells. Nature Communications, 5:5293, 2014. 61

[121] Jinhua Gao, Wei Gao, Xiaoling Ma, Zhenghao Hu, Chunyu Xu, Xuelin Wang, Qiaoshi An, Chuluo Yang, Xiaoli Zhang, and Fujun Zhang. Over 14.5\% efficiency and $71.6 \%$ fill factor of ternary organic solar cells with $300 \mathrm{~nm}$ thick active layers. Energy and Environmental Science, 13(3):958-967, 2020. 61

[122] Lijiao Ma, Shaoqing Zhang, Huifeng Yao, Ye Xu, Jingwen Wang, Yunfei Zu, and Jianhui Hou. High-Efficiency Nonfullerene Organic Solar Cells Enabled by 1000 nm Thick Active Layers with a Low Trap-State Density. ACS Applied Materials and Interfaces, 12(16):18777-18784, 2020.

[123] Jianqiang Qin, Lixiu Zhang, Zuo Xiao, Shanshan Chen, Kuan Sun, Zhigang Zang, Chenyi Yi, Yongbo Yuan, Zhiwen Jin, Feng Hao, Yuanhang Cheng, Qinye Bao, and Liming Ding. Over 16\% efficiency from thick-film organic solar cells. Science Bulletin, 65(23):1979-1982, 2020. 61

[124] Alexander J. Gillett, Alberto Privitera, Rishat Dilmurat, Akchheta Karki, Deping Qian, Anton Pershin, Giacomo Londi, William K. Myers, Jaewon Lee, Jun Yuan, Seo-Jin Ko, Moritz K. Riede, Feng Gao, Guillermo C. Bazan, Akshay Rao, ThucQuyen Nguyen, David Beljonne, and Richard H. Friend. The role of charge recombination to spin-triplet excitons in non-fullerene acceptor organic solar cells. arXiv preprint arXiv:2010.10978, 2020. 62

[125] Andrej Classen, Christos L. Chochos, Larry Lüer, Vasilis G. Gregoriou, Jonas Wortmann, Andres Osvet, Karen Forberich, Iain McCulloch, Thomas Heumüller, 
and Christoph J. Brabec. The role of exciton lifetime for charge generation in organic solar cells at negligible energy-level offsets. Nature Energy, 5(9):711-719, 2020. 62

[126] Hyojung Cha, Jiaying Wu, Andrew Wadsworth, Jade Nagitta, Saurav Limbu, Sebastian Pont, Zhe Li, Justin Searle, Mark F. Wyatt, Derya Baran, Ji Seon Kim, Iain McCulloch, and James R. Durrant. An Efficient, "Burn in" Free Organic Solar Cell Employing a Nonfullerene Electron Acceptor. Advanced Materials, 29(33):1701156, 2017. 63

[127] Sunsun Li, Long Ye, Wenchao Zhao, Shaoqing Zhang, Subhrangsu Mukherjee, Harald Ade, and Jianhui Hou. Energy-Level Modulation of Small-Molecule Electron Acceptors to Achieve over 12\% Efficiency in Polymer Solar Cells. Advanced Materials, 28(42):9423-9429, 2016. 63

[128] L. E. Brus. Electron-electron and electron-hole interactions in small semiconductor crystallites: The size dependence of the lowest excited electronic state. The Journal of Chemical Physics, 80(9):4403-4409, 1984. 66

[129] Ardalan Armin, Jegadesan Subbiah, Martin Stolterfoht, Safa Shoaee, Zeyun Xiao, Shirong Lu, David J. Jones, and Paul Meredith. Reduced Recombination in High Efficiency Molecular Nematic Liquid Crystalline: Fullerene Solar Cells. Advanced Energy Materials, 6(22):1-10, 2016. 68, 97, 98, 102

[130] Bronson Philippa, Martin Stolterfoht, Paul L. Burn, Gytis Juška, Paul Meredith, Ronald D. White, and Almantas Pivrikas. The impact of hot charge carrier mobility on photocurrent losses in polymer-based solar cells. Scientific Reports, $4: 5695,2014.69$

[131] Martin Stolterfoht, Safa Shoaee, Ardalan Armin, Hui Jin, Ivan Kassal, Wei Jiang, Paul Burn, and Paul Meredith. Electric Field and Mobility Dependent FirstOrder Recombination Losses in Organic Solar Cells. Advanced Energy Materials, 7(4):1601379, 2017. 69, 105, 118

[132] Seyed Mehrdad Hosseini, Nurlan Tokmoldin, Young Woong Lee, Yingping Zou, Han Young Woo, Dieter Neher, and Safa Shoaee. Putting Order into PM6:Y6 


\section{BIBLIOGRAPHY}

Solar Cells to Reduce the Langevin Recombination in $400 \mathrm{~nm}$ Thick Junction. Solar RRL, 4(11):2000498, 2020. 70

[133] Nurlan Tokmoldin, Seyed Mehrdad Hosseini, Meysam Raoufi, Le Quang Phuong, Oskar J Sandberg, Huilan Guan, Yingping Zou, Dieter Neher, and Safa Shoaee. Extraordinarily long diffusion length in PM6:Y6 organic solar cells. Journal of Materials Chemistry A, 8(16):7854-7860, 2020. 70

[134] Leif A.A. Pettersson, Lucimara S. Roman, and Olle Inganäs. Modeling photocurrent action spectra of photovoltaic devices based on organic thin films. Journal of Applied Physics, 86(1):487-496, 1999. 73

[135] George F. Burkhard, Eric T. Hoke, and Michael D. McGehee. Accounting for interference, scattering, and electrode absorption to make accurate internal quantum efficiency measurements in organic and other thin solar cells. Advanced Materials, 22(30):3293-3297, 2010. 73

[136] Jiaying Wu, Joel Luke, Harrison Ka Hin Lee, Pabitra Shakya Tuladhar, Hyojung Cha, Soo-Young Young Jang, Wing Chung Tsoi, Martin Heeney, Hongkyu Kang, Kwanghee Lee, Thomas Kirchartz, Ji-Seon Seon Kim, and James R. Durrant. Tail state limited photocurrent collection of thick photoactive layers in organic solar cells. Nature Communications, 10(1):5159, 2019. 73, 123

[137] Ardalan Armin, Aren Yazmaciyan, Mike Hambsch, Jun Li, Paul L. Burn, and Paul Meredith. Electro-Optics of Conventional and Inverted Thick Junction Organic Solar Cells. ACS Photonics, 2(12):1745-1754, 2015. 75

[138] Dieter Neher, Juliane Kniepert, Arik Elimelech, and L. Jan Anton Koster. A New Figure of Merit for Organic Solar Cells with Transport-limited Photocurrents. Scientific Reports, 6, 2016. 76

[139] Dieter Neher, Juliane Kniepert, Arik Elimelech, and L. Jan Anton Koster. A New Figure of Merit for Organic Solar Cells with Transport-limited Photocurrents. Scientific Reports, 6:24861, 2016. 76, 77, 101 
[140] Juliane Kniepert, Ilja Lange, Jan Heidbrink, Jona Kurpiers, Thomas J.K. Brenner, L. Jan Anton Koster, and Dieter Neher. Effect of solvent additive on generation, recombination, and extraction in PTB7:PCBM solar cells: A conclusive experimental and numerical simulation study. Journal of Physical Chemistry C, 119(15):8310-8320, 2015. 80

[141] Steve Albrecht, Wolfram Schindler, Jona Kurpiers, Juliane Kniepert, James C. Blakesley, Ines Dumsch, Sybille Allard, Konstantinos Fostiropoulos, Ullrich Scherf, and Dieter Neher. On the field dependence of free charge carrier generation and recombination in blends of PCPDTBT/PC 70BM: Influence of solvent additives. Journal of Physical Chemistry Letters, 3(5):640-645, 2012.

[142] Juliane Kniepert, Marcel Schubert, James C. Blakesley, and Dieter Neher. Photogeneration and recombination in P3HT/PCBM solar cells probed by time-delayed collection field experiments. Journal of Physical Chemistry Letters, 2(7):700-705, 2011. 80

[143] B. Bernardo, D. Cheyns, B. Verreet, R. D. Schaller, B. P. Rand, and N. C. Giebink. Delocalization and dielectric screening of charge transfer states in organic photovoltaic cells. Nature Communications, 5(3245), 2014. 80

[144] Askat E. Jailaubekov, Adam P. Willard, John R. Tritsch, Wai Lun Chan, Na Sai, Raluca Gearba, Loren G. Kaake, Kenrick J. Williams, Kevin Leung, Peter J. Rossky, and X. Y. Zhu. Hot charge-transfer excitons set the time limit for charge separation at donor/acceptor interfaces in organic photovoltaics. Nature Materials, 12(1):66-73, 2013. 80

[145] Maria Saladina, Pablo Simón Marqués, Anastasia Markina, Safakath Karuthedath, Christopher Wöpke, Clemens Göhler, Yue Chen, Magali Allain, Philippe Blanchard, Clément Cabanetos, Denis Andrienko, Frédéric Laquai, Julien Gorenflot, and Carsten Deibel. Charge Photogeneration in Non-Fullerene Organic Solar Cells: Influence of Excess Energy and Electrostatic Interactions. Advanced Functional Materials, 31(8):2007479, 2021. 80 


\section{BIBLIOGRAPHY}

[146] Safakath Karuthedath, Julien Gorenflot, Yuliar Firdaus, Neha Chaturvedi, Catherine S.P. De Castro, George T. Harrison, Jafar I. Khan, Anastasia Markina, Ahmed H. Balawi, Top Archie Dela Peña, Wenlan Liu, Ru Ze Liang, Anirudh Sharma, Sri H.K. Paleti, Weimin Zhang, Yuanbao Lin, Erkki Alarousu, Dalaver H. Anjum, Pierre M. Beaujuge, Stefaan De Wolf, Iain McCulloch, Thomas D. Anthopoulos, Derya Baran, Denis Andrienko, and Frédéric Laquai. Intrinsic efficiency limits in low-bandgap non-fullerene acceptor organic solar cells. Nature Materials, 20(3):378-384, 2021. 80

[147] Jafar I. Khan, Maha A. Alamoudi, Neha Chaturvedi, Raja S. Ashraf, Mohammed N. Nabi, Anastasia Markina, Wenlan Liu, Top Archie Dela Peña, Weimin Zhang, Olivier Alévêque, George T. Harrison, Wejdan Alsufyani, Eric Levillain, Stefaan De Wolf, Denis Andrienko, Iain McCulloch, and Frédéric Laquai. Impact of Acceptor Quadrupole Moment on Charge Generation and Recombination in Blends of IDT-Based Non-Fullerene Acceptors with PCE10 as Donor Polymer. Advanced Energy Materials, 11(28):2100839, 2021. 80

[148] Martin Stolterfoht, Ardalan Armin, Safa Shoaee, Ivan Kassal, Paul Burn, and Paul Meredith. Slower carriers limit charge generation in organic semiconductor light-harvesting systems. Nature Communications, 7:11944, 2016. 81

[149] G. Grancini, M. Maiuri, D. Fazzi, A. Petrozza, H. J. Egelhaaf, D. Brida, G. Cerullo, and G. Lanzani. Hot exciton dissociation in polymer solar cells. Nature Materials, 12(1):29-33, 2013. 81

[150] Safa Shoaee, Florent Deledalle, Pabitra Shakya Tuladhar, Ravichandran Shivanna, Sridhar Rajaram, K. S. Narayan, and James R. Durrant. A comparison of charge separation dynamics in organic blend films employing fullerene and perylene diimide electron acceptors. Journal of Physical Chemistry Letters, 6(1):201$205,2015$.

[151] Yufei Zhong, Martina Causa', Gareth John Moore, Philipp Krauspe, Bo Xiao, Florian Günther, Jonas Kublitski, Rishi Shivhare, Johannes Benduhn, Eyal BarOr, Subhrangsu Mukherjee, Kaila M. Yallum, Julien Réhault, Stefan C.B. 
Mannsfeld, Dieter Neher, Lee J. Richter, Dean M. DeLongchamp, Frank Ortmann, Koen Vandewal, Erjun Zhou, and Natalie Banerji. Sub-picosecond chargetransfer at near-zero driving force in polymer:non-fullerene acceptor blends and bilayers. Nature Communications, 11(1):833, 2020. 81

[152] Uli Würfel, Lorena Perdigón-Toro, Jona Kurpiers, Christian M. Wolff, Pietro Caprioglio, Jeromy James Rech, Jingshuai Zhu, Xiaowei Zhan, Wei You, Safa Shoaee, Dieter Neher, and Martin Stolterfoht. Recombination between photogenerated and electrode-induced charges dominates the fill factor losses in optimized organic solar cells. Journal of Physical Chemistry Letters, 10(12):3473-3480, 2019. 81, 89, 98, 102

[153] Stefan Zeiske, Oskar J. Sandberg, Nasim Zarrabi, Wei Li, Paul Meredith, and Ardalan Armin. Direct observation of trap-assisted recombination in organic photovoltaic devices. Nature Communications, 12(1):3603, 2021. 81, 121

[154] Martin Stolterfoht, Ardalan Armin, Bronson Philippa, and Dieter Neher. The Role of Space Charge Effects on the Competition between Recombination and Extraction in Solar Cells with Low-Mobility Photoactive Layers. Journal of Physical Chemistry Letters, 7(22):4716-4721, 2016. 81, 97, 98, 105, 118

[155] Ardalan Armin, James R. Durrant, and Safa Shoaee. Interplay between triplet-, singlet-charge transfer states and free charge carriers defining bimolecular recombination rate constant of organic solar cells. Journal of Physical Chemistry C, 121(25):13969-13976, 2017. 81

[156] Andreas Paulke, Samuel D. Stranks, Juliane Kniepert, Jona Kurpiers, Christian M. Wolff, Natalie Schön, Henry J. Snaith, Thomas J.K. Brenner, and Dieter Neher. Charge carrier recombination dynamics in perovskite and polymer solar cells. Applied Physics Letters, 108(11):113505, 2016. 81

[157] Elisa Collado-Fregoso, Silvina N. Pugliese, Mariusz Wojcik, Johannes Benduhn, Eyal Bar-Or, Lorena Perdigón Toro, Ulrich Hörmann, Donato Spoltore, Koen Vandewal, Justin M. Hodgkiss, and Dieter Neher. Energy-gap law for photocurrent generation in fullerene-based organic solar cells: The case of low-donor- 
content blends. Journal of the American Chemical Society, 141(6):2329-2341, 2019. 81

[158] R. Österbacka, K. Genevičius, A. Pivrikas, G. Juška, K. Arlauskas, T. Kreouzis, D. D.C. Bradley, and H. Stubb. Quantum efficiency and initial transport of photogenerated charge carriers in $\pi$-conjugated polymers. Synthetic Metals, 139(3):811-813, 2003. 81

[159] Jona Kurpiers and Dieter Neher. Dispersive non-geminate recombination in an amorphous polymer:fullerene blend. Scientific Reports, 6(26832):1-10, 2016. 83, 152

[160] Juliane Kniepert, Andreas Paulke, Lorena Perdigón-Toro, Jona Kurpiers, Huotian Zhang, Feng Gao, Jun Yuan, Yingping Zou, Vincent M. Le Corre, L. Jan Anton Koster, and Dieter Neher. Reliability of charge carrier recombination data determined with charge extraction methods. Journal of Applied Physics, 126(20):205501, 2019. 85

[161] Steven A. Hawks, Benjamin Y. Finck, and Benjamin J. Schwartz. Theory of current transients in planar semiconductor devices: Insights and applications to organic solar cells. Physical Review Applied, 3(4):044014, 2015.

[162] Oskar J. Sandberg, Kristofer Tvingstedt, Paul Meredith, and Ardalan Armin. Theoretical perspective on transient photovoltage and charge extraction techniques. Journal of Physical Chemistry C, 123(23):14261-14271, 2019. 85, 87, 97, 123

[163] Uli Würfel and Moritz Unmüssig. Apparent field-dependence of the charge carrier generation in organic solar cells as a result of (bimolecular) recombination. Solar RRL, 2(10):1800229, 2018. 89, 92

[164] R. Sokel and R. C. Hughes. Numerical analysis of transient photoconductivity in insulators. Journal of Applied Physics, 53(11):7414-7424, 1982. 89, 102

[165] Davide Bartesaghi, Irene Del Carmen Pérez, Juliane Kniepert, Steffen Roland, Mathieu Turbiez, Dieter Neher, and L. Jan Anton Koster. Competition between recombination and extraction of free charges determines the fill factor of organic solar cells. Nature Communications, 6(7083):1-10, 2015. 96 
[166] Frédéric Laquai, Denis Andrienko, Carsten Deibel, and Dieter Neher. Charge carrier generation, recombination, and extraction in polymer-fullerene bulk heterojunction organic solar cells. In Advances in Polymer Science, volume 272, pages 267-291. Springer, Cham, 2017. 96

[167] Christopher M. Proctor, Martijn Kuik, and Thuc Quyen Nguyen. Charge carrier recombination in organic solar cells. Progress in Polymer Science, 38(12):19411960, 2013. 96, 97

[168] David Kiermasch, Andreas Baumann, Mathias Fischer, Vladimir Dyakonov, and Kristofer Tvingstedt. Revisiting lifetimes from transient electrical characterization of thin film solar cells; A capacitive concern evaluated for silicon, organic and perovskite devices. Energy and Environmental Science, 11(3):629-640, 2018. 97, 123

[169] Wolfgang Tress, Karl Leo, and Moritz Riede. Dominating recombination mechanisms in organic solar cells based on $\mathrm{ZnPc}$ and C60. Applied Physics Letters, 102(16):163901, 2013. 97, 123

[170] Aren Yazmaciyan, Martin Stolterfoht, Paul L. Burn, Qianqian Lin, Paul Meredith, and Ardalan Armin. Recombination Losses Above and Below the Transport Percolation Threshold in Bulk Heterojunction Organic Solar Cells. Advanced Energy Materials, 8(18):1703339, 2018. 97, 118

[171] Martin Stolterfoht, Bronson Philippa, Safa Shoaee, Hui Jin, Wei Jiang, Ronald D. White, Paul L. Burn, Paul Meredith, and Almantas Pivrikas. Charge Transport without Recombination in Organic Solar Cells and Photodiodes. Journal of Physical Chemistry C, 119(48):26866-26874, 2015.

[172] Safa Shoaee, Martin Stolterfoht, and Dieter Neher. The Role of Mobility on Charge Generation, Recombination, and Extraction in Polymer-Based Solar Cells. Advanced Energy Materials, 8(28):1703355, 2018. 97

[173] Martin Stolterfoht, Ardalan Armin, Bronson Philippa, Ronald D. White, Paul L. Burn, Paul Meredith, Gytis Juška, and Almantas Pivrikas. Photocarrier drift distance in organic solar cells and photodetectors. Scientific Reports, 5:9949, 2015. 98 


\section{BIBLIOGRAPHY}

[174] Alvin M. Goodman and Albert Rose. Double extraction of uniformly generated electron-hole pairs from insulators with noninjecting contacts. Journal of Applied Physics, 42(7):2823-2830, 1971. 105

[175] Sebastian Wilken, Oskar J. Sandberg, Dorothea Scheunemann, and Ronald Österbacka. Watching Space Charge Build up in an Organic Solar Cell. Solar RRL, 4(3):1900505, 2020. 105

[176] V. D. Mihailetchi, J. Wildeman, and P. W.M. Blom. Space-charge limited photocurrent. Physical Review Letters, 94(12):126602, 2005. 105

[177] Paula Hartnagel and Thomas Kirchartz. Understanding the Light-Intensity Dependence of the Short-Circuit Current of Organic Solar Cells. Advanced Theory and Simulations, 3(10):2000116, 2020. 108

[178] Thomas Kirchartz and Jenny Nelson. Meaning of reaction orders in polymer:fullerene solar cells. Physical Review B, 86(16):165201, 2012. 108, 123

[179] Oskar J. Sandberg, Staffan Dahlström, Mathias Nyman, Sebastian Wilken, Dorothea Scheunemann, and Ronald Österbacka. Impact of a Doping-Induced Space-Charge Region on the Collection of Photogenerated Charge Carriers in Thin-Film Solar Cells Based on Low-Mobility Semiconductors. Physical Review Applied, 12(3):034008, 2019. 109, 112

[180] Guangzheng Zuo, Mathieu Linares, Tanvi Upreti, and Martijn Kemerink. General rule for the energy of water-induced traps in organic semiconductors. Nature Materials, 18(6):588-593, 2019. 117, 122, 134, 135

[181] Yuanbao Lin, Begimai Adilbekova, Yuliar Firdaus, Emre Yengel, Hendrik Faber, Muhammad Sajjad, Xiaopeng Zheng, Emre Yarali, Akmaral Seitkhan, Osman M. Bakr, Abdulrahman El-Labban, Udo Schwingenschlögl, Vincent Tung, Iain McCulloch, Frédéric Laquai, and Thomas D. Anthopoulos. 17\% Efficient Organic Solar Cells Based on Liquid Exfoliated WS2 as a Replacement for PEDOT:PSS. Advanced Materials, 31(46):1902965, 2019. 122

[182] Zhaomiyi Zeng, Zhiming Zhong, Wenkai Zhong, Jiaxin Zhang, Lei Ying, Gang Yu, Fei Huang, and Yong Cao. High-detectivity organic photodetectors based 
on a thick-film photoactive layer using a conjugated polymer containing a naphtho[1,2-: C:5,6- c] bis[1,2,5]thiadiazole unit. Journal of Materials Chemistry C, 7(20):6070-6076, 2019. 122

[183] Dezhi Yang, Xiaokang Zhou, and Dongge Ma. Fast response organic photodetectors with high detectivity based on rubrene and C60. Organic Electronics: physics, materials, applications, 14(11):3019-3023, 2013. 122

[184] Ross D. Jansen-Van Vuuren, Almantas Pivrikas, Ajay K. Pandey, and Paul L. Burn. Colour selective organic photodetectors utilizing ketocyanine-cored dendrimers. Journal of Materials Chemistry C, 1(22):3532-3543, 2013. 122

[185] Naresh B. Kotadiya, Anirban Mondal, Paul W.M. Blom, Denis Andrienko, and Gert Jan A.H. Wetzelaer. A window to trap-free charge transport in organic semiconducting thin films. Nature Materials, 18(11):1182-1186, 2019. 122

[186] H. T. Nicolai, M. Kuik, G. A.H. Wetzelaer, B. De Boer, C. Campbell, C. Risko, J. L. Brédas, and P. W.M. Blom. Unification of trap-limited electron transport in semiconducting polymers. Nature Materials, 11(10):882-887, 2012. 122

[187] Ardalan Armin, Safa Shoaee, Qianqian Lin, Paul L. Burn, and Paul Meredith. On the unipolarity of charge transport in methanofullerene diodes. npj Flexible Electronics, 1(1), 2017. 122

[188] G.-J. A. H. Wetzelaer, M. Kuik, M. Lenes, and P. W.M. Blom. Origin of the dark-current ideality factor in polymer:fullerene bulk heterojunction solar cells. Applied Physics Letters, 99(15):153506, 2011. 123

[189] R. A. Street, M. Schoendorf, A. Roy, and J. H. Lee. Interface state recombination in organic solar cells. Physical Review B, 82(20):205307, 2010.

[190] Thomas Kirchartz, Bart E. Pieters, James Kirkpatrick, Uwe Rau, and Jenny Nelson. Recombination via tail states in polythiophene:fullerene solar cells. Physical Review B, 83(11):115209, 2011.

[191] R. A. Street. Localized state distribution and its effect on recombination in organic solar cells. Physical Review B - Condensed Matter and Materials Physics, 84(7):075208, 2011. 


\section{BIBLIOGRAPHY}

[192] Daniel Rauh, Carsten Deibel, and Vladimir Dyakonov. Charge density dependent nongeminate recombination in organic bulk heterojunction solar cells. Advanced Functional Materials, 22(16):3371-3377, 2012.

[193] Qinye Bao, Oskar Sandberg, Daniel Dagnelund, Simon Sandén, Slawomir Braun, Harri Aarnio, Xianjie Liu, Weimin M. Chen, Ronald Österbacka, and Mats Fahlman. Trap-Assisted recombination via integer charge transfer states in organic bulk heterojunction photovoltaics. Advanced Functional Materials, 24(40):6309-6316, 2014. 123

[194] Mohammed Azzouzi, Philip Calado, Andrew M. Telford, Flurin Eisner, Xueyan Hou, Thomas Kirchartz, Piers R.F. Barnes, and Jenny Nelson. Overcoming the Limitations of Transient Photovoltage Measurements for Studying Recombination in Organic Solar Cells. Solar RRL, 4(5):1900581, 2020. 123

[195] Erin L. Ratcliff, Jens Meyer, K. Xerxes Steirer, Neal R. Armstrong, Dana Olson, and Antoine Kahn. Energy level alignment in PCDTBT:PC70BM solar cells: Solution processed $\mathrm{NiOx}$ for improved hole collection and efficiency. Organic Electronics, 13(5):744-749, 2012. 132, 156

[196] Guangzheng Zuo, Zhaojun Li, Olof Andersson, Hassan Abdalla, Ergang Wang, and Martijn Kemerink. Molecular Doping and Trap Filling in Organic Semiconductor Host-Guest Systems. Journal of Physical Chemistry C, 121(14):7767-7775, 2017. 134

[197] Thomas Kirchartz. Influence of diffusion on space-charge-limited current measurements in organic semiconductors. Beilstein Journal of Nanotechnology, 4:180$188,2013$.

[198] Jason A. Röhr, Davide Moia, Saif A. Haque, Thomas Kirchartz, and Jenny Nelson. Exploring the validity and limitations of the Mott-Gurney law for chargecarrier mobility determination of semiconducting thin-films. Journal of Physics Condensed Matter, 30(10):105901, 2018. 134

[199] Yu Yan, Wei Li, Feilong Cai, Jinlong Cai, Zhiwei Huang, Robert S. Gurney, Dan Liu, David G. Lidzey, Andrew J. Pearson, and Tao Wang. Correlating Nanoscale Morphology with Device Performance in Conventional and Inverted 
PffBT4T-2OD:PC71BM Polymer Solar Cells. ACS Applied Energy Materials, $1(7): 3505-3512,2018.136$

[200] Ning Li and Christoph J. Brabec. Air-processed polymer tandem solar cells with power conversion efficiency exceeding 10\%. Energy and Environmental Science, 8(10):2902-2909, 2015.

[201] Wanjung Kim, Jung Kyu Kim, Eunchul Kim, Tae Kyu Ahn, Dong Hwan Wang, and Jong Hyeok Park. Conflicted effects of a solvent additive on PTB7:PC71BM bulk heterojunction solar cells. Journal of Physical Chemistry C, 119(11):59545961, 2015. 136

[202] Martin Stolterfoht, Pietro Caprioglio, Christian M. Wolff, José A. Márquez, Joleik Nordmann, Shanshan Zhang, Daniel Rothhardt, Ulrich Hörmann, Yohai Amir, Alex Redinger, Lukas Kegelmann, Fengshuo Zu, Steve Albrecht, Norbert Koch, Thomas Kirchartz, Michael Saliba, Thomas Unold, and Dieter Neher. The impact of energy alignment and interfacial recombination on the internal and external open-circuit voltage of perovskite solar cells. Energy and Environmental Science, 12(9):2778-2788, 2019. 153

[203] Wenchao Zhao, Sunsun Li, Shaoqing Zhang, Xiaoyu Liu, and Jianhui Hou. Ternary Polymer Solar Cells based on Two Acceptors and One Donor for Achieving 12.2\% Efficiency. Advanced Materials, 29(2), 2017. 156

[204] Wenchao Zhao, Sunsun Li, Huifeng Yao, Shaoqing Zhang, Yun Zhang, Bei Yang, and Jianhui Hou. Molecular Optimization Enables over 13\% Efficiency in Organic Solar Cells. Journal of the American Chemical Society, 139(21):7148-7151, 2017. 156

[205] Zhan'Ao Tan, Shusheng Li, Fuzhi Wang, Deping Qian, Jun Lin, Jianhui Hou, and Yongfang Li. High performance polymer solar cells with as-prepared zirconium acetylacetonate film as cathode buffer layer. Scientific Reports, 4:1-9, 2015. 156

[206] Shaoqing Zhang, Long Ye, Wenchao Zhao, Delong Liu, Huifeng Yao, and Jianhui Hou. Side chain selection for designing highly efficient photovoltaic polymers with 2D-conjugated structure. Macromolecules, 47(14):4653-4659, 2014. 156 
[207] Hao Zhang, Huifeng Yao, Junxian Hou, Jie Zhu, Jianqi Zhang, Wanning Li, Runnan Yu, Bowei Gao, Shaoqing Zhang, and Jianhui Hou. Over 14\% Efficiency in Organic Solar Cells Enabled by Chlorinated Nonfullerene Small-Molecule Acceptors. Advanced Materials, 30(28):1-7, 2018. 156 